\author{
Aus dem Fachbereich Medizin \\ der Johann Wolfgang Goethe-Universität \\ Frankfurt am Main \\ betreut am \\ Zentrum der Gesundheitswissenschaften \\ Institut für Arbeits-, Sozial- und Umweltmedizin \\ Direktor: Prof. Dr. David Groneberg
}

\title{
Der Einfluss eines systematischen Krafttrainings auf die objektive ergonomische Arbeitsweise von Zahnärzten und Zahnmedizinischen Fachangestellten
}

\author{
Dissertation \\ zur Erlangung des Doktorgrades der Zahnmedizin \\ des Fachbereichs Medizin \\ der Johann Wolfgang Goethe-Universität \\ Frankfurt am Main
}

vorgelegt von

Tim Florian Weis

aus Bonn

Frankfurt am Main, 2021 
Dekan:

Referentin:

Korreferent/in:

Tag der mündlichen Prüfung:
Prof. Dr. Stefan Zeuzem

PD. Dr. Dr. Daniela Ohlendorf

Prof. Dr. Stefan Rüttermann

25.10.2021 


\section{Inhaltsverzeichnis}

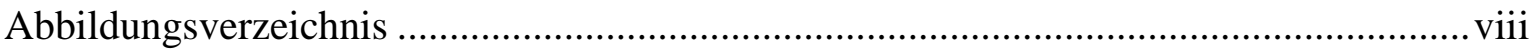

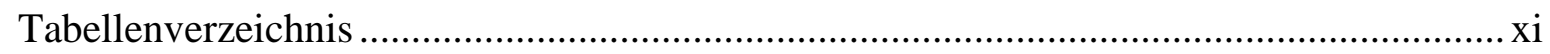

Abkürzungsverzeichnis ......................................................................................

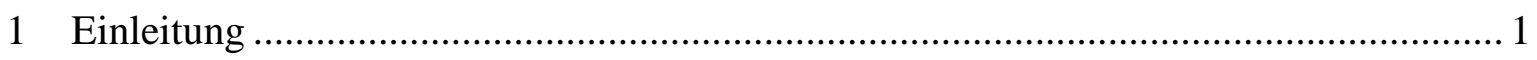

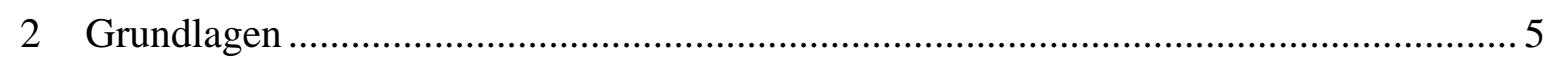

2.1 Funktionelle Anatomie des Stütz- und Bewegungsapparats unter Berücksichtigung ihrer Halte- und Stabilisierungsfunktion ..................................................................... 5

2.1.1 Funktionelle Anatomie der Wirbelsäule und der Rückenmuskulatur .................. 6

2.1.2 Funktionelle Anatomie der Bauchwand und der Bauchmuskulatur.................... 8

2.1.3 Funktionelle Anatomie der Schultern und der Schultermuskulatur ................... 10

2.2 Gesundheitliche Beeinträchtigungen des Stütz- und Bewegungsapparats in Form von Muskuloskelettalen Erkrankungen .

2.3 Berufsbild von Zahnarzt und Zahnmedizinischen Fachangestellten unter besonderer Berücksichtigung gesundheitlicher Risikofaktoren ..................................... 15

2.4 Prävalenz muskuloskelettaler Konditionen unter Zahnärzten und Zahnmedizinischen Fachangestellten ................................................................... 20

2.5 Ergonomie in der Zahnmedizin ....................................................................... 23

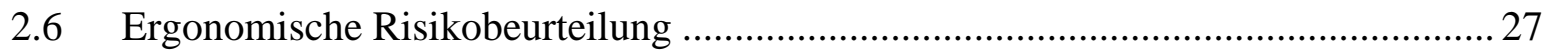

2.7 Krafttraining und seine Relevanz in der Zahnmedizin ......................................... 30

2.8 Aktueller Literaturstand bezüglich der Auswirkung eines systematischen Krafttrainings auf die ergonomische Arbeitsweise bei Zahnärzten und Zahnmedizinischen

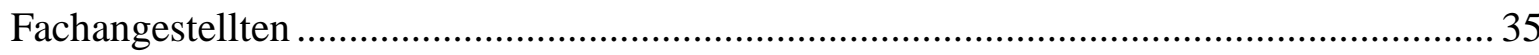

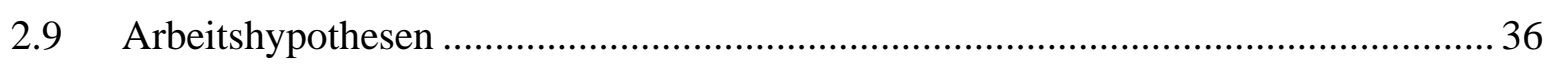

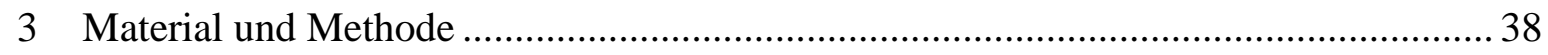




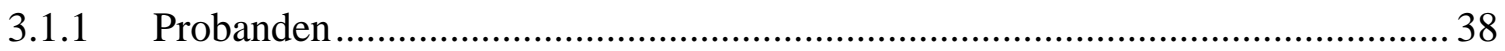

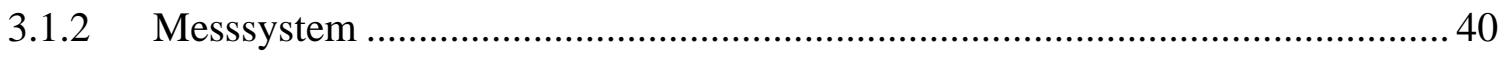

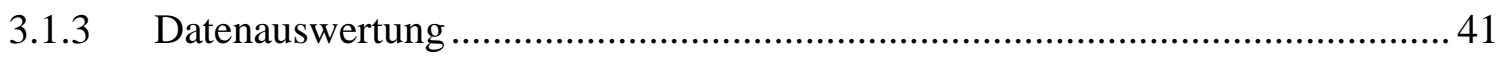

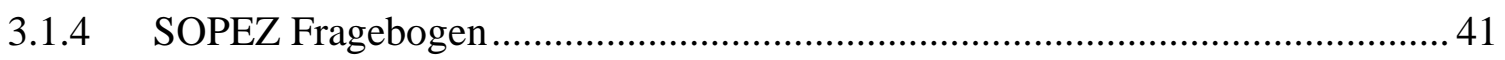

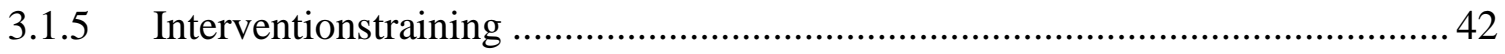

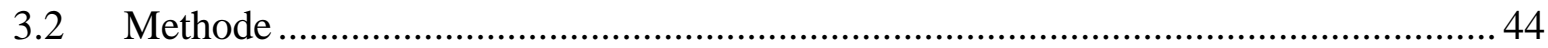

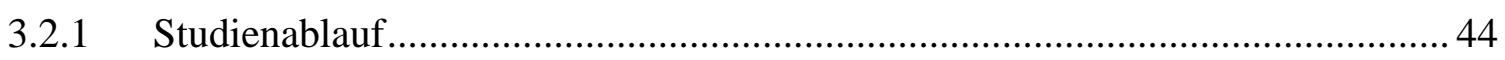

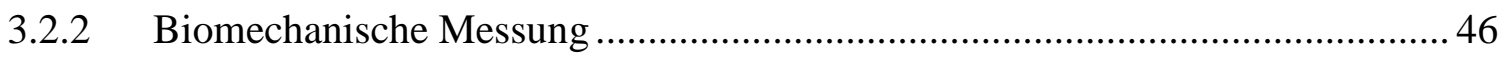

3.2.3 Übungen zur Kräftigung der stabilisierten Haltemuskulatur ........................... 49

3.2.4 Auswertung der kinematischen Daten........................................................ 53

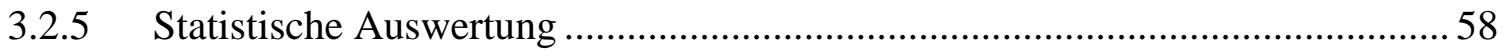

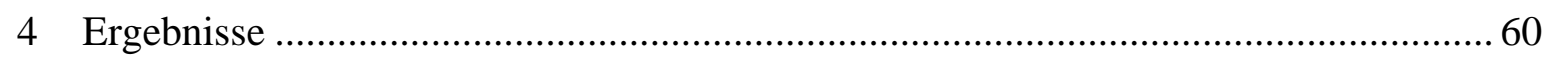

4.1 Prä-Post-Auswertung der Medianwerte und Quartilsabstände................................60

4.1.1 Prä-Post-Auswertung der Medianwerte und Quartilsabstände - Oberarm, Unterarm, Handgelenk \& Rotation linke Körperhälfte

4.1.2 Prä-Post-Auswertung der Medianwerte und Quartilsabstände - Oberarm, Unterarm, Handgelenk \& Rotation rechte Körperhälfte

4.1.3 Prä-Post-Auswertung der Medianwerte und Quartilsabstände - Oberarm, Unterarm, Handgelenk \& Rotation im Seitenvergleich

4.1.4 Prä-Post-Auswertung der Medianwerte und Quartilsabstände - Nacken und Oberkörper

4.1.5 Prä-Post-Auswertung der Medianwerte und Quartilsabstände - RULA Gesamtscore

4.1.6 Prä-Post-Auswertung der Medianwerte und Quartilsabstände Berufsgruppenvergleich 68 
4.2 Prä-Post-Auswertung der Bewertungsparameter nach relativer zeitlicher Verteilung.

4.2.1 Prä-Post-Auswertung der Bewertungsparameter nach relativer zeitlicher Verteilung - Oberarm linksseitig

4.2.2 Prä-Post-Auswertung der Bewertungsparameter nach relativer zeitlicher Verteilung - Unterarm linksseitig

4.2.3 Prä-Post-Auswertung der Bewertungsparameter nach relativer zeitlicher Verteilung - Handgelenk \& Rotation linksseitig ....

4.2.4 Prä-Post-Auswertung der Bewertungsparameter nach relativer zeitlicher Verteilung - Oberarm rechtsseitig

4.2.5 Prä-Post-Auswertung der Bewertungsparameter nach relativer zeitlicher Verteilung - Unterarm rechtsseitig

4.2.6 Prä-Post-Auswertung der Bewertungsparameter nach relativer zeitlicher Verteilung - Handgelenk \& Rotation rechtsseitig ....

4.2.7 Prä-Post-Auswertung der Bewertungsparameter nach relativer zeitlicher Verteilung - Nacken

4.2.8 Prä-Post-Auswertung der Bewertungsparameter nach relativer zeitlicher Verteilung - Oberkörper .

4.2.9 Prä-Post-Auswertung der Bewertungsparameter nach relativer zeitlicher Verteilung - RULA Gesamtscore

4.3 Prä-Post-Auswertung der riskanten Bewertungsparameter nach relativer zeitlicher Verteilung und Berufsgruppe

4.4 Prä-Post-Auswertung der Gelenkwinkel und Gelenkpositionen nach relativer zeitlicher Verteilung

4.4.1 Prä-Post-Auswertung der Gelenkwinkel nach relativer zeitlicher Verteilung -

Oberarm linksseitig

4.4.2 Prä-Post-Auswertung der Gelenkwinkel/-position nach relativer zeitlicher Verteilung - Unterarm linksseitig 
4.4.3 Prä-Post-Auswertung der Gelenkwinkel nach relativer zeitlicher Verteilung Handgelenk \& Rotation linksseitig

4.4.4 Prä-Post-Auswertung der Gelenkwinkel nach relativer zeitlicher Verteilung -

Oberarm rechtsseitig .88

4.4.5 Prä-Post-Auswertung der Gelenkwinkel nach relativer zeitlicher Verteilung Unterarm rechtsseitig

4.4.6 Prä-Post-Auswertung der Gelenkwinkel nach relativer zeitlicher Verteilung Handgelenk \& Rotation rechtsseitig 93

4.4.7 Prä-Post-Auswertung der Gelenkwinkel nach relativer zeitlicher Verteilung Nacken 94

4.4.8 Prä-Post-Auswertung der Gelenkwinkel nach relativer zeitlicher Verteilung Oberkörper

5 Diskussion 101

5.1 Die ergonomische Arbeitsweise von Zahnärzten und Zahnmedizinischen Fachangestellten

5.1.1 Ergebnisanalyse: Ergonomische Arbeitsweise von ZÄ und ZFAs anhand der relativen zeitlichen Verteilung von Gelenkwinkeln und Gelenkpositionen.

5.1.2 Die ergonomische Arbeitsweise unter Berücksichtigung ergonomischer Vorgaben aus der Zahnmedizin.

5.1.3 Die ergonomische Arbeitsweise unter Berücksichtigung des Berufsbilds von Zahnärzten und Zahnmedizinischen Fachangestellten 108

5.2 Die ergonomische Risikobeurteilung von Zahnärzten und Zahnmedizinischen Fachangestellten....

5.2.1 Ergebnisanalyse: ergonomische Risikobeurteilung von ZÄ und ZFAs anhand RULA Scores und ihrer relativen zeitlichen Verteilung

5.2.2 Die ergonomische Risikobeurteilung und die Prävalenz Muskuloskelettaler

Erkrankungen von Zahnärzten und Zahnmedizinischen Fachangestellten 
5.3 Einfluss eines systematischen Krafttrainings auf die ergonomische Arbeitsweise von Zahnärzten und Zahnmedizinischen Fachangestellten

5.3.1 Ergebnisanalyse und Hypothesenprüfung: Einfluss eines systematischen

Krafttrainings auf die Gelenkwinkel und Gelenkpositionen.

5.3.2 Ergebnisanalyse und Hypothesenprüfung: Einfluss eines systematischen

Krafttrainings auf die ergonomische Risikobeurteilung

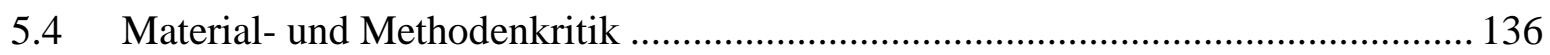

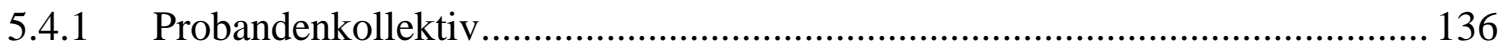

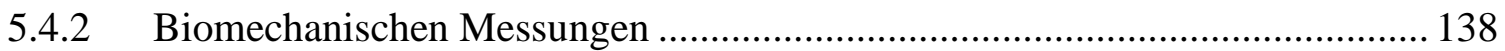

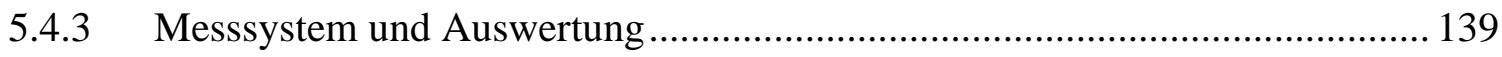

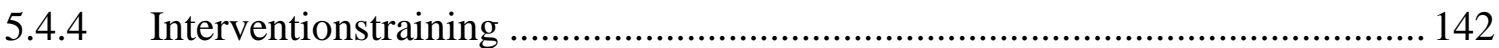

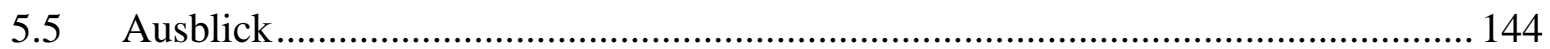

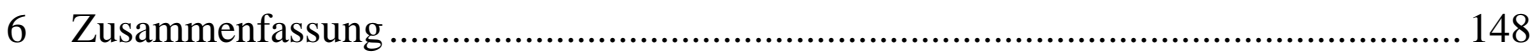

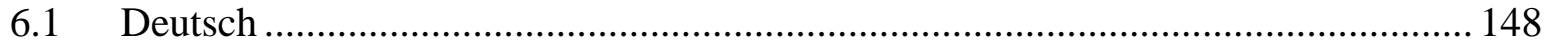

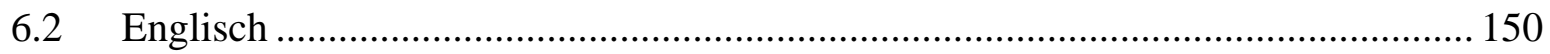

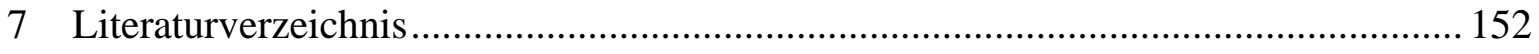

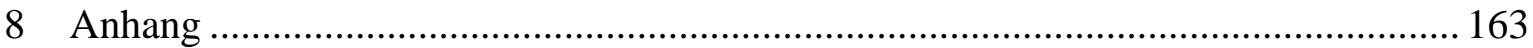

8.1 Probanden-Information und Einwilligungserklärung ........................................ 163

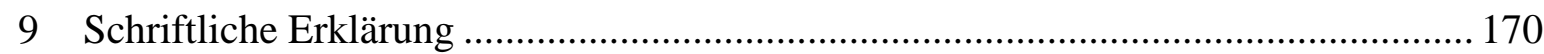




\section{Abbildungsverzeichnis}

Abbildung 1: Wirbelkörper, Zwischenwirbelscheiben und Bänder der Wirbelsäule............ 7

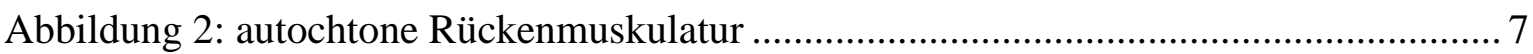

Abbildung 3: vordere und seitliche Bauchmuskulatur .................................................. 10

Abbildung 4: Gelenke der Schulter im Überblick .......................................................... 12

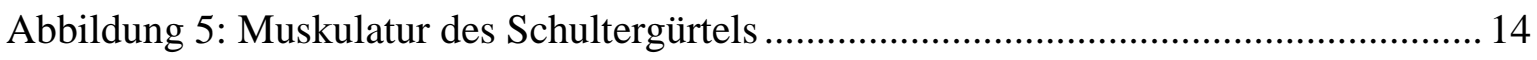

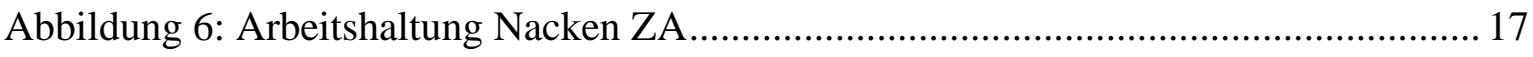

Abbildung 7: Verteilung Probandenkollektiv nach Berufsgruppe …................................ 38

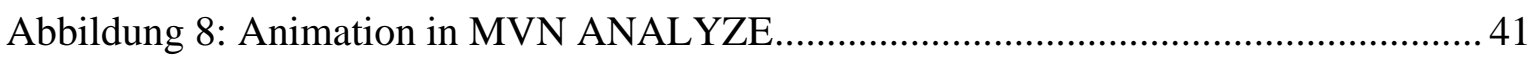

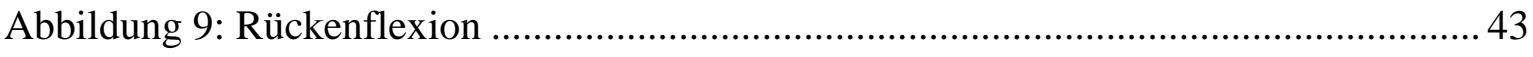

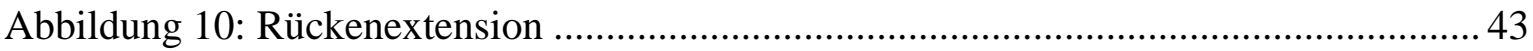

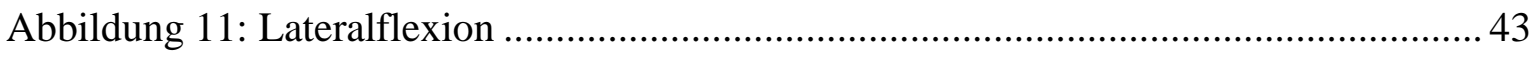

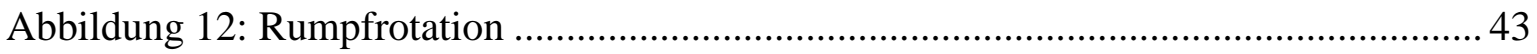

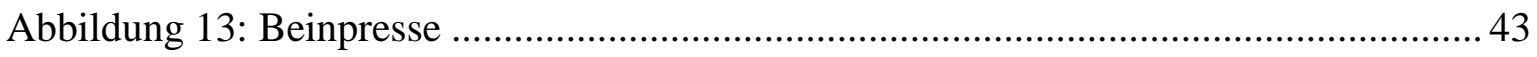

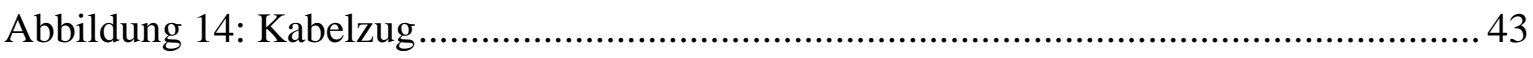

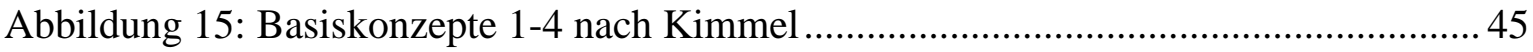

Abbildung 16: Behandlungssimulation Phantomkopf..................................................... 46

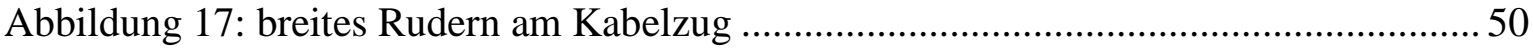

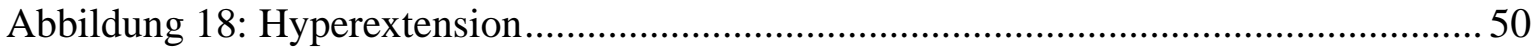

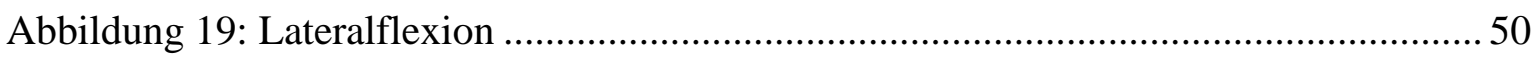

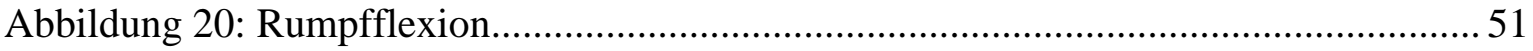

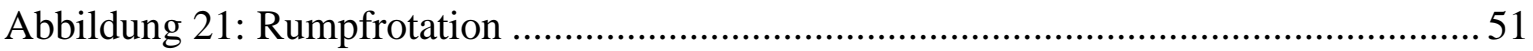

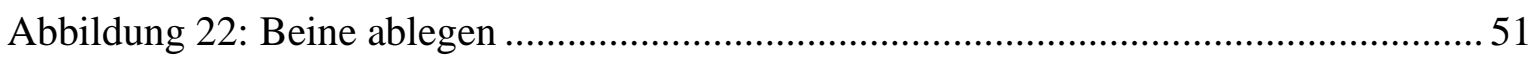

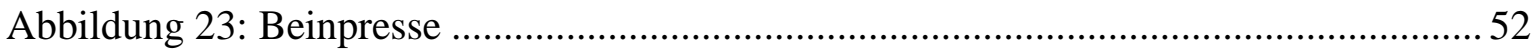




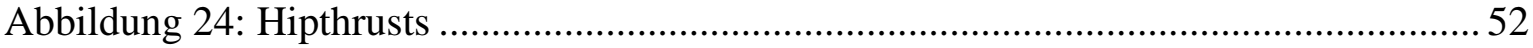

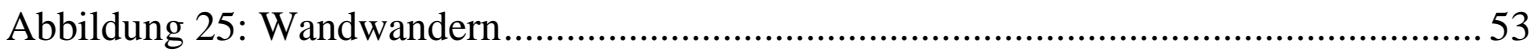

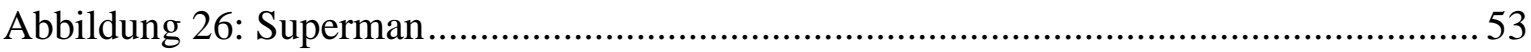

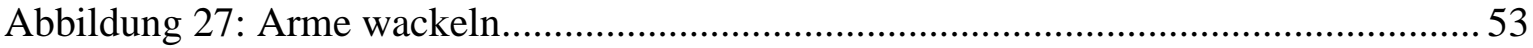

Abbildung 28: RULA Employee Assessment Worksheet................................................ 56

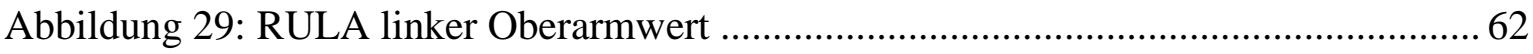

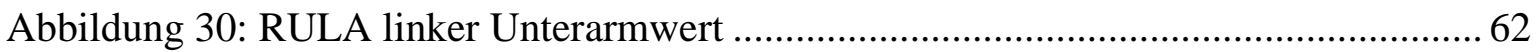

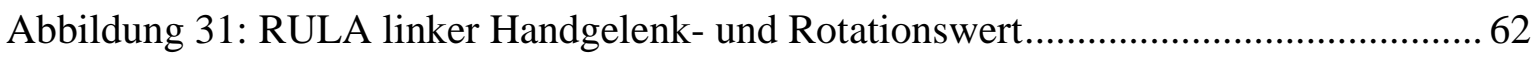

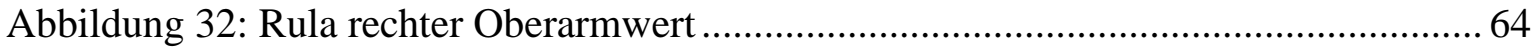

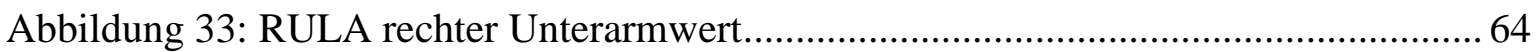

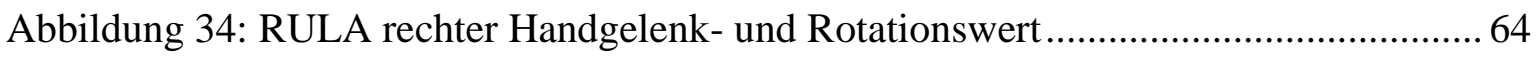

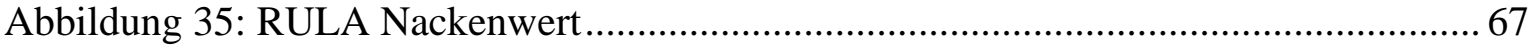

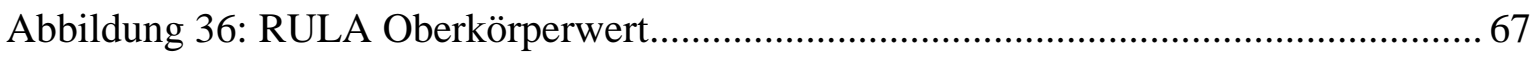

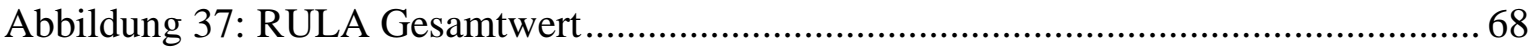

Abbildung 38: Proz. Zeitanteile: RULA Oberarm linksseitig .......................................... 72

Abbildung 39: Proz. Zeitanteile: RULA Unterarm linksseitig........................................ 73

Abbildung 40: Prozentuelle Zeitanteile: RULA Handgelenk \& Rotation linksseitig ......... 74

Abbildung 41: Proz. Zeitanteile: RULA Oberarm rechtsseitig ....................................... 75

Abbildung 42: Proz. Zeitanteile: RULA Unterarm rechtsseitig ...................................... 76

Abbildung 43: Proz. Zeitanteile: RULA Handgelenk \& Rotation rechtsseitig ................... 77

Abbildung 44: Proz. Zeitanteile: RULA Nacken ........................................................ 78

Abbildung 45: Prozentuelle Zeitanteile: RULA Oberkörper ........................................... 79

Abbildung 46: Proz. Zeitanteile: RULA Gesamtscore …................................................. 80

Abbildung 47: Proz. Zeitanteile RULA riskante Nackenwerte für alle Probanden ............ 81 
Abbildung 48: Proz. Zeitanteile RULA riskante Nackenwerte für Zahnärzte

Abbildung 49: Gelenkwinkel Oberarm linksseitig - Flexion/Extension ............................ 84

Abbildung 50: Gelenkwinkel Oberarm linksseitig - Elevation Schulter ........................... 84

Abbildung 51: Gelenkwinkel Oberarm linksseitig - Abduktion ..................................... 85

Abbildung 52: Gelenkwinkel Unterarm linksseitig - Flexion/Extension .......................... 86

Abbildung 53: Gelenkposition Unterarm linksseitig - Position Transversalebene .............. 86

Abbildung 54: Gelenkwinkel Handgelenk linksseitig - Flexion/Extension ...................... 88

Abbildung 55: Gelenkwinkel Handgelenk linksseitig - Deviation .................................. 88

Abbildung 56: Gelenkwinkel Oberarm rechtsseitig - Flexion/Extension .......................... 90

Abbildung 57: Gelenkwinkel Oberarm rechtsseitig - Elevation Schulter .......................... 90

Abbildung 58: Gelenkwinkel Oberarm rechtsseitig - Abduktion..................................... 91

Abbildung 59: Gelenkwinkel Unterarm rechtsseitig - Flexion/Extension.......................... 92

Abbildung 60: Gelenkposition Unterarm rechtsseitig - Position Transversalebene ........... 92

Abbildung 61: Gelenkwinkel Handgelenk linksseitig - Flexion/Extension ....................... 94

Abbildung 62: Gelenkwinkel Handgelenk linksseitig - Deviation ................................... 94

Abbildung 63: Gelenkwinkel Nacken - Flexion/Extension ............................................ 96

Abbildung 64: Gelenkwinkel Nacken - Rotation ........................................................... 96

Abbildung 65: Gelenkwinkel Nacken - Beugung (Frontalebene) .................................... 97

Abbildung 66: Gelenkwinkel Oberkörper - Flexion/Extension...................................... 99

Abbildung 67: Gelenkwinkel Oberkörper - Rotation .................................................... 99

Abbildung 68: Gelenkwinkel Oberkörper - Beugung (Frontalebene) ............................. 100 


\section{Tabellenverzeichnis}

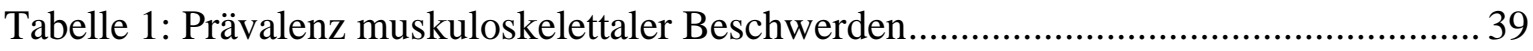

Tabelle 2: Angaben zu Rücken-/Ergonomieschulung, Sport bzw. Training in Freizeit...... 39

Tabelle 3: RULA Scores - linker Oberarm, Unterarm, Handgelenk \& Rotation ................. 61

Tabelle 4: RULA Scores - rechter Oberarm, Unterarm, Handgelenk \& Rotation .............. 63

Tabelle 5: RULA Scores - Median- und Quartilwerte: Nacken und Oberkörper................. 66

Tabelle 6: RULA Scores - Median- und Quartilwerte: Gesamtwerte .................................68

Tabelle 7: RULA Scores ZÄ - links Oberarm, Unterarm, Handgelenk \& Rotation ........... 69

Tabelle 8: RULA Scores Z ̈̈ - rechts Oberarm, Unterarm, Handgelenk \& Rotation........... 70

Tabelle 9: Tabelle 5: RULA Scores ZÄ - Nacken und Oberkörper .................................... 70

Tabelle 10: RULA Scores Z ̈̈ - Median und Quartilsabstand: Gesamtwerte ..................... 71

Tabelle 11: Prozentuelle Zeitanteile: RULA Oberarm linksseitig .................................... 71

Tabelle 12: Prozentuelle Zeitanteile: RULA Unterarm linksseitig ................................... 72

Tabelle 13: Prozentuelle Zeitanteile: RULA Handgelenk \& Rotation linksseitig .............. 73

Tabelle 14: Prozentuelle Zeitanteile: RULA Oberarm rechtsseitig................................... 74

Tabelle 15: Prozentuelle Zeitanteile: RULA Unterarm rechtsseitig ................................. 75

Tabelle 16: Prozentuelle Zeitanteile: RULA Handgelenk \& Rotation rechtsseitig.............. 76

Tabelle 17: Prozentuelle Zeitanteile: RULA Nacken...................................................... 77

Tabelle 18: Prozentuelle Zeitanteile: RULA Oberkörper.................................................... 78

Tabelle 19: Prozentuelle Zeitanteile: RULA Gesamtscore ............................................. 79 


\section{Abkürzungsverzeichnis}

\begin{tabular}{|c|c|}
\hline $1 \mathrm{RM}$ & one repetition maximum \\
\hline ATP & Adenosintriphosphat \\
\hline BAuA & Bundesanstalt für Arbeitsschutz und Arbeitsmedizin \\
\hline DGUV & Deutsche Gesetzliche Unfallversicherung \\
\hline DIN & Deutsche Industrie Norm \\
\hline ERAT & ergonomic risk assesment tools \\
\hline IEA & International Ergonomics Association \\
\hline ISO & International Organization for Standardization \\
\hline KSL & KOLMOGOROFF-SMIRNOFF-LILLIEFORS-TEST \\
\hline Lig. & Ligamentum \\
\hline LMM & Leitmerkmalmethoden \\
\hline M-DOPAI & Modified-Dental Operator Posture Assessement Instrument \\
\hline M. & Musculus \\
\hline Mm. & Musculi \\
\hline OSHA & Occupational Safety and Health Administration \\
\hline OWAS & Ovako Working posture Analysis \\
\hline QEC & Quick Exposure Check \\
\hline RULA & Rapid Upper Limp Assessment \\
\hline $\begin{array}{l}\text { SARS- } \\
\text { CoV-2 }\end{array}$ & severe acute respiratory syndrome coronavirus type 2 \\
\hline SPM & Systematic Parametic Mapping \\
\hline stat. & statistisch \\
\hline WHO & World Health Organisation \\
\hline $\mathrm{ZÄ}$ & Zahnärzte \\
\hline ZFAs & Zahnmedizinische Fachangestellte \\
\hline$\omega$ & Winkelgeschwindigkeiten \\
\hline
\end{tabular}




\section{$1 \quad$ Einleitung}

Eine Studie aus den USA im Jahr 2015[1] verglich 974 Berufsgruppen bezüglich ihres Einflusses auf die menschliche Gesundheit. Dabei wurden die Daten des „Occupational Information Network“, eines US-amerikanischen Arbeitsministeriums verwendet, welche definierte Gesundheitsrisiken für die einzelnen Berufsgruppen bewertete. Im direkten Vergleich wurde die Berufsgruppe von Zahnärzten (ZÄ) und Zahnmedizinischen Fachangestellten (ZFAs) als die gesundheitsschädlichste Berufsgruppe identifiziert. Wobei als die größten Gesundheitsrisiken in dieser Berufssparte, Exposition gegenüber Schadstoffen, Krankheiten und Infektionen sowie prolongierte Arbeitszeit im Sitzen aufgeführt wurden.[1] Die Zahnmedizin dient der Prävention, Diagnose und Therapie von Zahn-, Mund- und Kiefererkrankungen. Das schwerpunktmäßige Arbeitsumfeld der zahnmedizinischen Professionen ist folglich der Patientenmund. Die stark begrenzten räumlichen Verhältnisse, die durch die Mundöffnung eingeschränkten Sicht- und Lichtverhältnisse sowie der Speichelfluss und sonstige anatomische Spezifitäten des Patienten stellen an ZÄ und ZFAs besondere Arbeitsbedingungen. Die Arbeit im Patientenmund erfordert eine sehr hohe Präzision, für die ein übersichtliches und sauberes Arbeitsfeld unerlässlich ist, was eine hohe Koordination zwischen ZÄ und ZFAs erfordert.[2-5]

Die Gegebenheiten des Arbeitsumfelds stellen an diese Berufsgruppe enorme physische Anforderungen.[3, 6-8] Während der Behandlung ist die Bewegungsfreiheit aufgrund des kleinen Arbeitsfelds oftmals erheblich eingeschränkt. Um eine ausreichende Arbeitspräzision gewährleisten zu können, nehmen Z $\ddot{A}$ und ZFAs oftmals statische Körperhaltungen ein, die über lange Zeit während der Behandlung oder während des Arbeitstages gehalten werden müssen. Dabei kommt es oft zur Einnahme von gesundheitlich ungeeigneten Haltungen und Zwangshaltungen.[3, 4, 6, 8-11] Um außerdem eine ausreichende Visualisierung der Mundhöhle zu erzielen nehmen die Arbeiter in zahnmedizinischen Professionen oftmals krumme, vorgebeugte Haltungen ein, bei denen es zusätzlich oftmals $\mathrm{zu}$ einer seitlichen Beugung und Rotation von Nacken und Oberkörper kommt. Diese Bewegungs- und Arbeitsmuster können dabei zur Entwicklung von Pathologien und Ungleichgewichten innehrlab der Muskulatur führen.[3, 4, 8, 10, 12-14] Um z.B. Instrumente oder anderes Equipment während der Behandlung zu halten, arbeiten ZÄ und ZFAs häufig über lange Zeit mit angehobenen und abduzierten Armen, mit der Folge hoher statischer Arbeitsbelastungen der Nacken- und Schulterregion.[7, 12, 15, 16] Des Weite- 
ren muss für das Halten und Arbeiten mit dentalen Instrumenten zum Teil mit erheblichem Kraftaufwand gearbeitet werden, welche in Hand- und Handgelenksproblemen resultieren können.[4, 17-19]

Diese Arbeitsweisen stehen z.T. im erheblichen Kontrast zu dem, was in der Zahnmedizin als ergonomisch ideal betrachtet wird bzw. empfohlen ist. Als ergonomisch ideal gilt in der modernen Zahnmedizin eine sitzende, aktive, aufrechte und symmetrische Arbeitshaltung. Dabei sollte die Körperhaltung während der Arbeit möglichst entspannt sein.[2025] Seit vielen Jahren ist bekannt, dass die Prävalenz muskuloskelettaler Beschwerden unter Z̈̈ und ZFAs sehr hoch ist.[6, 10, 12, 18, 26-28] Besonders die Körperregionen des Nackens, des Rückens und der Schultern scheinen besonders betroffen.[6, 10, 12, 18 , 26, 29-31] Infolge ist diese Berufsgruppe einem erhöhten Risiko ausgesetzt an Krankheitsbildern, wie z.B. Myalgien, Bandscheibendegeneration, dem „Tension Neck Syndrom“ oder auch dem „Carpal-Tunnel-Syndrom“ zu erkranken. [3, 17, 32, 33] In der Zahnmedizin hat die Forschung und Lehre der Ergonomie als Hauptaufgabe dieser hohen Prävalenz von Muskuloskelettalen Erkrankungen und Beschwerden präventiv entgegen zu wirken. Es lassen sich dabei verhaltenspräventive sowie verhältnispräventive Maßnahmen unterscheiden, die die ergonomische Arbeitsweise von Z̈̈ und ZFAs verbessern sollen. Die aktuelle Literatur der ergonomischen Zahnmedizin beschäftigt sich allerdings vorwiegend mit den verhältnispräventiven Maßnahmen, wobei z.B. der Einfluss von Stühlen, Behandlungseinheiten, Instrumentarium oder Lupenbrillen und Mikroskopen untersucht wird.[34-37] Obwohl Studien [7, 38] den positiven Einfluss dieser gestalterischen Anpassungen des Arbeitsumfelds bestätigen konnten, ist die Prävalenz muskuloskelettaler Beschwerden unter ZÄ und ZFAs weiterhin sehr hoch. Der tatsächliche Einfluss verhältnispräventiver Maßnahmen wird deshalb von manchen Autoren $[7,38]$ in Frage gestellt.

Verhaltenspräventive Maßnahmen werden bei Z̈̈ und ZFA hingegen in der aktuellen Literatur kaum untersucht. Die vorhandene Literatur [3, 21, 22, 24] beschränkt sich dabei größtenteils auf den Einfluss der Arbeitshaltung bzw. wie die ergonomische Arbeitshaltung und Arbeitsweise am Patienten anzupassen ist, um muskuloskelettaler Beschwerden vorzubeugen. Zwar ist allgemein anerkannt, dass körperliche Aktivität zu einer verbesserten Gesundheit führt und auch mit einer verringerten Prävalenz Muskuloskelettaler Erkrankungen verknüpft ist.[8, 38-40] Allerdings fehlen experimentelle Daten die diesen 
Einfluss in den beiden eben genannten Berufsgruppen bestätigen. Obwohl unterschiedliche Sportarten (z.B.: Aerobic, Yoga, Krafttraining) als adäquate präventive Maßnahme für zahnmedizinische Berufe bezeichnet werden, konnten sich auch diese Empfehlungen bisher nicht auf experimentelle Daten stützen.[5, 24, 41] Da selbst im Begriff Muskuloskelettale Erkrankung die Muskulatur an erster Stelle steht ist es wenig verständlich, warum der Einfluss der Muskulatur auf die ergonomische Arbeitsweise und die Prävalenz muskuloskelettaler Beschwerden in der Zahnmedizin kaum untersucht sind.

In der Sportwissenschaft ist bekannt, dass die Entwicklung einer gut ausgeprägten Muskulatur Voraussetzung für jegliche Form körperlicher Aktivität ist. Die Muskulatur als Stützkorsett dient aus präventiver Sicht der Entlastung und verringert die Verletzungsgefahr des Halte- und Stützapparats.[42, 43] Ein auf Muskelwachstum ausgelegtes systematisches Krafttraining konnte bereits vielfach für Berufe (z.B.: Büroangestellte, Laborarbeiter, Krankenhauspersonal), die der Zahnmedizin in Arbeitshaltung- und Arbeitsbelastung verwandt sind, zeigen, dass es die Prävalenz muskuloskelettaler Beschwerden unter den Probanden verringern und außerdem akute sowie chronische Schmerzen lindern konnte.[44-49] Wenige Daten finden sich hingegen in der wissenschaftlichen Literatur über den Einfluss von körperlichem Training als verhaltenspräventive Maßnahme auf die ergonomische Arbeitsweise, welche als Ursache für das Auftreten von Muskuloskelettalen Erkrankungen angenommen wird.[50] Von den bisherigen ergonomischen Untersuchungen, die den Einfluss von verhaltens- und verhältnispräventiven Maßnahmen erforschten, stützten sich des Weiteren nur sehr wenige auf objektive Daten.[37, 51] Vielmehr herrschten bisher subjektive Beurteilungen durch den Untersucher oder Eigenangaben des Probanden als Mittel zu Datenerhebung und -auswertung vor.[46-49, 52, 53] In dieser Forschungsarbeit soll besonders der Einfluss eines systematischen Krafttrainings als verhaltenspräventive Maßnahme auf die ergonomische Arbeitsweise von Zahnärzten und Zahnmedizinischen Fachangestellten untersucht werden. Die ergonomische Arbeitsweise wird anhand objektiver Daten analysiert und ausgewertet, um somit einen genauen Einblick in die zugrundeliegende Biomechanik zu bekommen. Auch die Beurteilung des ergonomischen Risikos der Arbeitshaltung wird analysiert und ausgewertet, um dieses anhand objektiver Daten zu belegen. In diesem Zusammenhang sollen anhand der gewonnen objektiven Daten Rückschlüsse gezogen werden, ob ein systematisches Krafttraining auch für die zahnmedizinischen Berufe adäquat ist und in wie fern sich die ergonomische Arbeitsweise durch verhaltenspräventive Maßnahmen beeinflussen lässt. 
Die Forschungsarbeit ist Teil der SOPEZ Studie zur Optimierung der Ergonomie in der Zahnarztpraxis.[54] 


\section{Grundlagen}

\subsection{Funktionelle Anatomie des Stütz- und Bewegungsapparats unter Berücksichti- gung ihrer Halte- und Stabilisierungsfunktion}

Der Stütz- und Bewegungsapparat des Menschen ist unterteilt in einen aktiven und einen passiven Teil. Der passive Stützapparat besteht aus dem menschlichen Skelett-, sowie den Skelettverbindungen, die sich in Gelenke und Bänder differenzieren.[55-57]

Die Skelettverbindungen lassen sich unterteilen in Gelenke und Bänder (Lig). Gelenke ermöglichen es dem Skelett einzelne Knochen in unterschiedliche Positionen zueinander zu bringen. Grundsätzlich werden drei Arten von Gelenkverbindungen (Bandgelenke, Knorpelgelenke, synoviale Gelenke) ${ }^{1}$ unterschieden, welche entsprechend der funktionellen Anforderungen im Körper vorkommen.[55-57] Bänder, welche zusätzlich an den beteiligten Knochen ansetzen und über das Gelenk verlaufen, stabilisieren dieses zusätzlich. Sie limitieren während der Bewegung den Bewegungsumfang, sorgen aber auch für erhöhte Stabilität.[55-57]

Das Skelett hat eine essenzielle Bedeutung für die Körperhaltung und die Ausführung von Körperbewegungen, indem es die Körperform vorgibt. Außerdem schützt es die im Körperinnern liegenden Organe.[55-57]

Der aktive Bewegungsapparat besteht aus der Skelettmuskulatur sowie den dazu gehörigen Sehnen und Faszien. Über seine Sehnen inseriert jeder Muskel an seinem i.d.R. proximalen fixen Ansatz und distalen mobilen Ursprung.[56, 57] Dabei verläuft er über ein Gelenk und verbindet die gelenkbildenden Knochen miteinander, wodurch die Muskelkraft auf das Skelett übertragen werden kann. Der Muskel verkürzt sich bei Kontraktion und über die inserierenden Sehnen können am Gelenk beteiligte Knochen in ihrer Position verändert oder auch stabilisiert werden. Die Skelettmuskulatur lässt sich über motorische Nervenfasern aus dem Gehirn oder Rückenmark steuern und dient somit der Ausführung gezielter Bewegungen.[56, 57] Des Weiteren leistet sie Haltearbeit und stabilisiert so das

\footnotetext{
${ }^{1}$ Als Bandgelenke werden gegeneinander bewegliche Knochen bezeichnet, die über Bindegewebe miteinander verbunden sind, wie z.B. am Unterarm zwischen Ulna und Radius.[56, 57] u.a. Knorpelgelenke verbinden gegeneinander bewegliche Knochen durch zwischen ihnen liegendes Knorpelgewebe. Diese Gelenke sind nur geringgradig oder kaum beweglich, z.B. die Symphyse des Beckens oder die Zwischenwirbelscheiben (Bandscheiben) der Wirbelsäule.[56, 57] Als „echte“ Gelenke werden synoviale Gelenke bezeichnet. Diese weisen zwei mit Knorpel überzogene Gelenkanteile auf, welche sich oftmals in Form von Gelenkkopf und Gelenkpfanne zusammenlagern. Sie weisen einen Gelenkspalt auf, welcher mit Gelenkflüssigkeit (Synovialis) gefüllt ist die als Gleitmittel dient, und sind außerdem von einer Gelenkkapsel umschlossen.[55-57] Zu den synovialen Gelenken zählen z.B. Schulter-, Knie-, Hüft- und Handgelenk.[55-57]
} 
Skelett, womit sie maßgeblich zur Körperhaltung beiträgt.[55, 56] Die Muskulatur lässt sich dabei unterteilen in Gruppen von Agonisten, welche der gleichen Bewegungsausführung dienen, und Antagonisten, die einer gegensätzlichen Bewegungsausführung dienen, sowie Synergisten, welche die Wirkung eines Agonisten unterstützen.[55, 56]

\subsubsection{Funktionelle Anatomie der Wirbelsäule und der Rückenmuskulatur}

Die Wirbelsäule besteht aus 24 freien sowie acht bis zehn verwachsenen Wirbelkörpern, welche einen ähnlichen Grundaufbau haben, aber an ihre lokalen Anforderungen anatomisch angepasst sind. Alle Wirbelkörper haben einen dorsal gelegenen Dornfortsatz (Processus Spinosus) und bilateral liegende Querfortsätze (Processi Transversi). Zwischen den freien Wirbelkörpern liegen die 23 Zwischenwirbelscheiben (Disci vertebrales), auch Bandscheiben genannt. Diese bestehen aus einem äußeren zugfesten Fasserring (Anulus fibrosus), welcher einen nicht-komprimierbaren Gallertkern (Nucleus pulposus) umschließt.[55-58] Funktionell dienen sie unter Belastung der Polsterung und dämpfen die Gewichts- und Druckbelastung, indem sie als „elastisches Wasserkissen“ den Druck gleichmäßig über die Wirbelkörper verteilen.[56-58] Des Weiteren erhöhen sie den Bewegungsspielraum der Wirbelsäule und verleihen ihr mehr Flexibilität.[56] Die einzelnen Wirbel stehen über Intervertebralgelenke miteinander in Verbindung, welche Rotationsund Flexionsbewegungen ermöglichen. Die Position der Wirbelkörper und der Intervertebralgelenke wird durch einen unmittelbar anliegenden Bandapparat stabilisiert (Abb.: 1).[55, 56] Die Wirbelsäule bildet das sogenannte Achsenskelett, welches mit Extremitäten und Rippen verbunden ist. Dabei überträgt sie das Gewicht von Kopf, Extremitäten sowie dem Rumpf über den Beckengürtel auf die Beine.[55, 56]

Die spezifische Form der menschlichen Wirbelsäule verlagert den Schwerpunkt der suprapelvinen Körpermaße über die Hüftgelenke. Dort kann sie mit einem Minimum an Kraftaufwand von der Muskulatur, insbesondere des Bauchs und Rückens, balanciert werden. Dadurch wird eine dauerhafte aufrechte Körperhaltung möglich.[56] Die Rückenmuskulatur hat somit entscheidende Bedeutung bei der Stabilisierung von Wirbelsäule und Rumpf. Unter Belastung kann sie die Haltung der Wirbelsäule absichern, indem sie manipulierenden Kräften durch Anspannung und somit Lagesicherung der einzelnen Wirbel entgegenwirkt. Die Rückenmuskulatur ermöglicht außerdem Bewegungen der Wirbelsäule, wie Extension, Rotation und Lateralflexion.[55-57] 
Sie lässt sich unterteilen in eine tiefer gelegene autochthone und eine oberflächlich liegende nicht-autochthone Rückenmuskulatur (Abb.: 2). Die autochthone Rückenmuskulatur lässt sich wiederum in medialen und lateralen Trakt unterteilen. Der laterale Trakt besteht aus sacrospinalem, spinotransversalem und intertransversalem System.[56, 57] Die Muskulatur des sacrospinalen Systems verbindet die Processi transversi der Wirbelkörper mit den Rippen und spielt somit eine Rolle bei der Dorsalextension und Lateralflexion des Rumpfes. Das spinotransversale System entspringt den Processi spinosi der Brustwirbel und inseriert an den Processi transversi der Halswirbel. Somit bewirken sie bei ipsilateraler Kontraktion eine Lateralflexion von Wirbelsäule, Kopf und Hals; bei bilateraler Kontraktion Dorsalflexion von Kopf und Hals. Das intertransversale System verbindet die Querfortsätze der Wirbel und führt bei einseitiger Kontraktion wiederum zur ipsilateralen Lateralflexion von Kopf und Hals.[56, 57] Der mediale Trakt der autochthonen Rückenmuskulatur besteht wiederum aus einem spinalen und einem transversospinalen System.[56-58] Die Muskulatur des spinalen Systems verläuft zwischen den Processi spinosi der Wirbelkörper und bewirkt somit eine Dorsalextension, sowie Lateralflexion der Wirbelsäule. Das transversospinale System verläuft zwischen den Processi transversi und den Processi spinosi der Wirbelkörper sowie dem Os occipitale des Schädels. Sie ermöglicht damit bei einseitiger Kontraktion eine ipsilaterale Flexion sowie Rotation und bei beidseitiger Kontraktion eine Dorsalextension bzw. Gelenkstabilisierung der Wirbelsäule bzw. des Kopfes.[56-58]

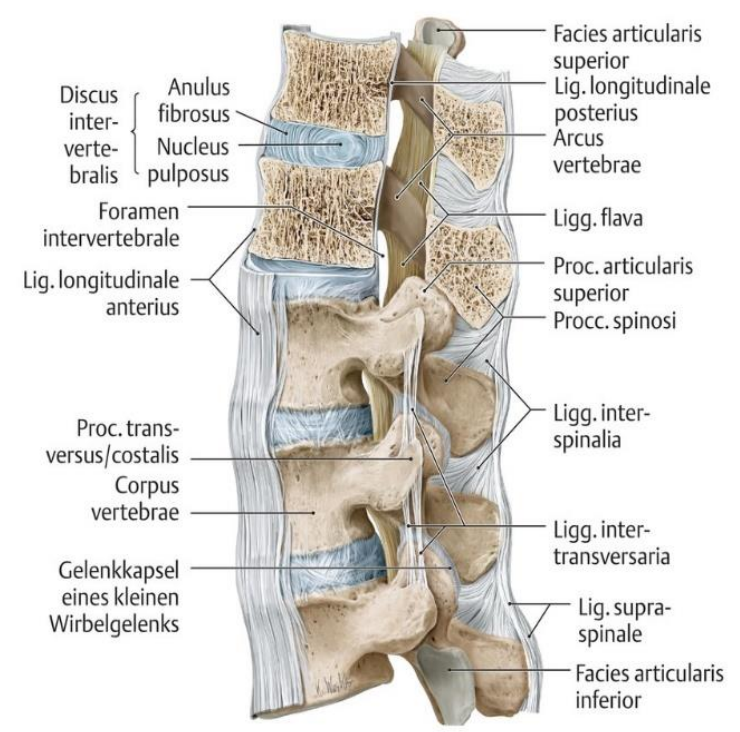

Abbildung 1: Wirbelkörper, Zwischenwirbelscheiben und Bänder der Wirbelsäule [56]

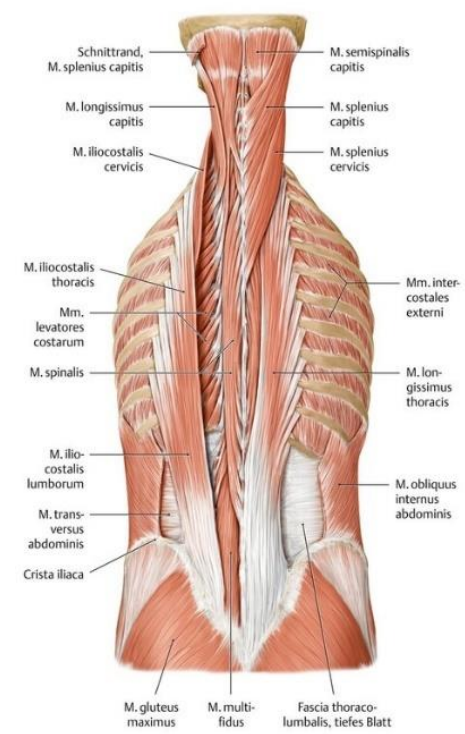

Abbildung 2: autochtone Rückenmuskulatur[58] 
Die nicht-autochthone Rückenmuskulatur, dient in ihrer Funktion nicht primär dem Aufrichten der Wirbelsäule, sondern vielmehr der Beweglichkeit der oberen Extremitäten. Sie verbindet die Wirbelsäule mit den oberen Extremitäten. Sie ist entwicklungsgeschichtlich eingewandert und liegt über der autochthonen Rückenmuskulatur relativ oberflächlich.[56-58] Abgesehen vom M. levator scapulae haben alle Muskeln ihren Ursprung an den Processi spinosi der Wirbelkörper. Nach ihrem Ansatz lassen sie sich in die spinokostalen Muskeln und spinoskapulären bzw. spinohumeralen Muskeln unterscheiden. Die spinokostalen Muskeln ziehen zu den Rippen und führen bei Kontraktion neben Lateralflexion, Dorsalextension auch zu einer Beeinflussung der Atemmechanik.[56-58]

Die spinoskapulären bzw spinohumeralen Muskeln setzen am Schulterblatt (Scapula) bzw. am proximalen Humerus an. Zu ihr gehören entsprechend vorwiegend die Muskulatur der Schultern und des Schultergürtels, wie z.B. M. levator scapulae, M. trapezius, M. latissimus dorsi, Mm. rhomboidei.[55-58] Ihre Funktion wird im Kapitel 2.1.2 näher erläutert.

\subsubsection{Funktionelle Anatomie der Bauchwand und der Bauchmuskulatur}

Die Bauchwand nimmt den unteren Teil der ventralen Rumpfwand ein.[57] Dabei erstreckt sich die ventrale und laterale Bauchwand von den Rippenbögen bis zur ventralen Christa iliaca (Darmbeinkamm), zur Symphyse der Schambeinkörper (Symphysis pubica) und dem Leistenband (Ligamentum inguinale).[56-58] Von der Bauchwand umschlossene Hohlräume umfassen außer der Bauchhöhle (Cavitas abdominalis) auch die Beckenhöhle (Cavitas pelvis) mit Becken- und Bauchorganen. Der Aufbau der Bauchwand ist grundlegend geprägt durch multiple Schichten von Bauchmuskulatur mit unterschiedlicher Verlaufsrichtung (Abb.: 3).[56-58] Die Bauchmuskulatur wirkt mit dem M. iliopsoas antagonistisch zur Rückenmuskulatur. Sie ermöglichen dadurch unter minimalem Kraftaufwand den Rumpf mit dem Schwerpunkt der suprapelvinen Körpermasse über den Hüftgelenken zu balancieren.[56]

Die Bauchmuskulatur lässt sich in seitliche, vordere und hintere Muskelgruppen unterteilen.[56-59] Zu den seitlichen Bauchmuskeln gehören M. obliquus externus abdominis, M. obliquus internus abdominis sowie M. transversus abdominis. Der M. obliquus abdominis entspringt den Rippenaußenflächen der 5. Bis 12. Rippe und setzt an der Christa 
iliaca und dem Lig. Inguinale an. Bei bilateraler Kontraktion bewirkt er ein Vorbeugen des Oberkörpers, eine einseitige Kontraktion führt zur ipsilateralen Seitneigung sowie kontralateralen Rotation. Der M. obliquus internus abdominis entspringt von der Fascia thoracolumbalis, der Christa iliaca sowie dem Lig. inguinale und hat seinen Ansatz an der 9. bis 12. Rippe. Entsprechend seines Verlaufs hat er die gleiche Funktion wie der M. obliquus abdominis.[56-58] Als letzter Muskel der seitlichen Bauchmuskulatur entspringt der M. transversus abdominis von der 7. bis 12. Rippe, der Fascia thoracolumbalis, der Christa iliaca sowie dem Lig. inguinale und findet seinen Ansatz am Os pubis und der Linea alba, einer Bindegewebsaponeurose. Bei einseitiger Kontraktion bewirkt er die Rotation des Rumpfes zur gleichen Seite.[56-58]

Die Fascia transversalis und die Aponeurosen der seitlichen Bauchmuskeln bilden gemeinsam die Rektusscheide in der der M. rectus abdominis liegt.[56, 57] Dieser gehört zur vorderen Bauchmuskulatur und entspringt dem 5. bis 7. Rippenknorpel sowie dem Proc. xiphoideus sterni. Der Muskel setzt am Os pubis auf beiden Seiten der Symphyse an und bewirkt somit funktionell ein Aufrichten bzw. Vorbeugen des Oberkörpers sowie ein ein Anheben des Beckens. Ebenfalls zur vorderen Bauchmuskulatur gehört der M. pyramidalis, welcher am Os pubis bilateral entspringt und an der Linea alba ansetzt. Bei Kontraktion spannt er die Linea alba.[56-59]

Zur Gruppe der hinteren Bauchmuskulatur zählen M. quadratus lumborum und M. psoas major.[56-59] Der M. quadratus lumborum entspringt der Crista iliaca sowie dem Lig. iliolumbale und setzt an der 12. Rippe und dem Proc. costalis der Lendenwirbelkörper an. Funktionell bewirkt er eine Seitneigung des Rumpfes. Der M. psoas major hat seinen Ursprung an den Wirbelkörpern der Lendenwirbelsäule sowie dem letzten Wirbel der Thoraxwirbelsäule und setzt am Oberschenkelknochen genauer dem Trochanter minor femoris an. Er bewirkt bei einer einseitigen Kontraktion eine ipsilaterale Seitneigung und bei beidseitiger Kontraktion ein Vorbeugen des Rumpfes.[56-58] 


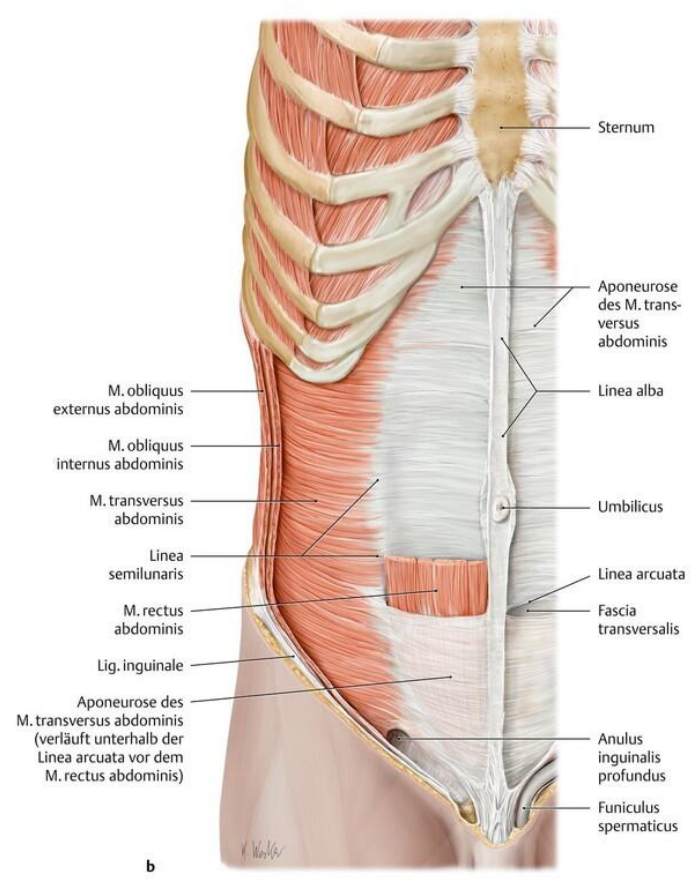

Abbildung 3: vordere und seitliche Bauchmuskulatur[58]

\subsubsection{Funktionelle Anatomie der Schultern und der Schultermuskulatur}

Die Aufgabe der Hand als Greiforgan erfordert von den oberen Extremitäten einen enormen Bewegungsumfang, um z.B. Tätigkeiten unter Kontrolle der Augen durchführen zu können. Diese Bewegungsspielraum wird zum einen gewährleistet durch Funktion des Schultergelenks und zum anderen durch die Beweglichkeit des Schultergelenks gegenüber dem Rumpf.[56]

Der Schultergürtel verbindet die oberen Extremitäten mit dem Thorax (Abb.: 4). Er besteht zum einen aus dem Schlüsselbein (Clavicula), welcher mit seinem sternalen Ende am Brustbein (Manubrium Sterni) und seinem akromialen Ende am Schulterblatt (Scapula) ansetzt und diese so beinahe waagerecht verbindet.[56, 57] Hier bildet es das mediale Schlüsselbeingelenk (Articulatio sternoclavicularis), die einzige echte gelenkige Verbindung des Schultergürtels zum Rumpf. Zum anderen besteht er aus der Scapula, einem platten Knochen mit dreieckigem Umriss, welcher drei Ränder (Margo medialis, lateralis, superior) und drei Ecken (Angulus superior, inferior und lateralis) hat. Das Schulterblatt hat außerdem zwei Seiten: die Facies costalis, welche flach der Rückwand des Brustkorbs aufgelagert ist und mit ihm das sogenannte ,Schulterblatt-Thorax-Gelenk“ bildet, sowie die nach außen gewandte Facies posterior, welche als Muskelursprungsfläche dient.[56] 
Des Weiteren weist das Schulterblatt zwei Knochenvorsprünge auf: Das massive Acromion überragt lateral sowie kranial die Scapula und die Gelenkhöhle des Schultergelenks. Es bildet mit der Clavicula das laterale Schlüsselbeingelenk (Articulatio acromioclavicularis). Der Processus corarciodeus erhebt sich lateral und ventral der Schultergelenkspfanne (Cavitas gelnoidalis). Beide Knochenfortsätze sind ligamentär über das Ligamentum coracoacromiale verbunden und bilden so das Schultergelenkdach (Fornix humeri).[56-58] Bei allen Bewegungen des Schultergürtels sind mediales, laterales Schlüsselbeingelenk sowie das „Schulterblatt-Thorax-Gelenk“ mechanisch gekoppelt. Die Art. sternoclavicularis ermöglicht dabei eine Bewegung der Clavicula mit befestigter Scapula um eine sagittale und vertikale Achse. Außerdem kommt es bei Elevation des Arms zwangsläufig zu einer Rotation der Clavicula um ihre Längsachse bis zu $30^{\circ}$. Das Gelenk ist somit für die Bewegung des Schulterblatts am Thorax entscheidend.[56] Die Art. acromioclavicularis sowie das „Schulterblatt-Thorax-Gelenk“ erlauben der Scapula außerdem eine Rotation um $60-70^{\circ}$ um eine Achse durch die Mitte des Schulterblatts, welche für Elevation und Adduktion des Arms wichtig ist. Die jeweiligen Freiheitsgrade werden dabei durch den Bandapparat des Schultergürtels eingeschränkt.[56, 57]

Das eigentliche Schultergelenk ist die Articulatio glenohumeralis. Sie wird einerseits gebildet zwischen der Gelenkpfanne der Scapula, die nach ventral/lateral ausgerichtet am Angulus lateralis liegt und klein sowie flach ist. Sowie andererseits dem Gelenkkopf (Caput humeri) des Oberarmknochens (Humerus). Der Humerus ist ein langer Röhrenknochen, dessen Gelenkkopf mit dem Schaft (Corpus humeri) einen Winkel von $135^{\circ}$ bildet. Das Caput humeri hat dabei eine Gelenkfläche in Halbkugelform mit 2,5-3cm Radius.[56] Beim Schultergelenk handelt es sich um ein Kugelgelenk mit drei Freiheitsgraden, welche Ante- und Retroversion, Ab- und Adduktion sowie Innen- und Außenrotation ermöglichen. Als „echtes“ Gelenk wird es von einer Gelenkkapsel umschlossen. Die dünne Gelenkkapsel wird des Weiteren durch einen Bandapparat verstärkt, der die Stabilität des Gelenks besonders nach ventral und kranial verstärkt.[56-58] 


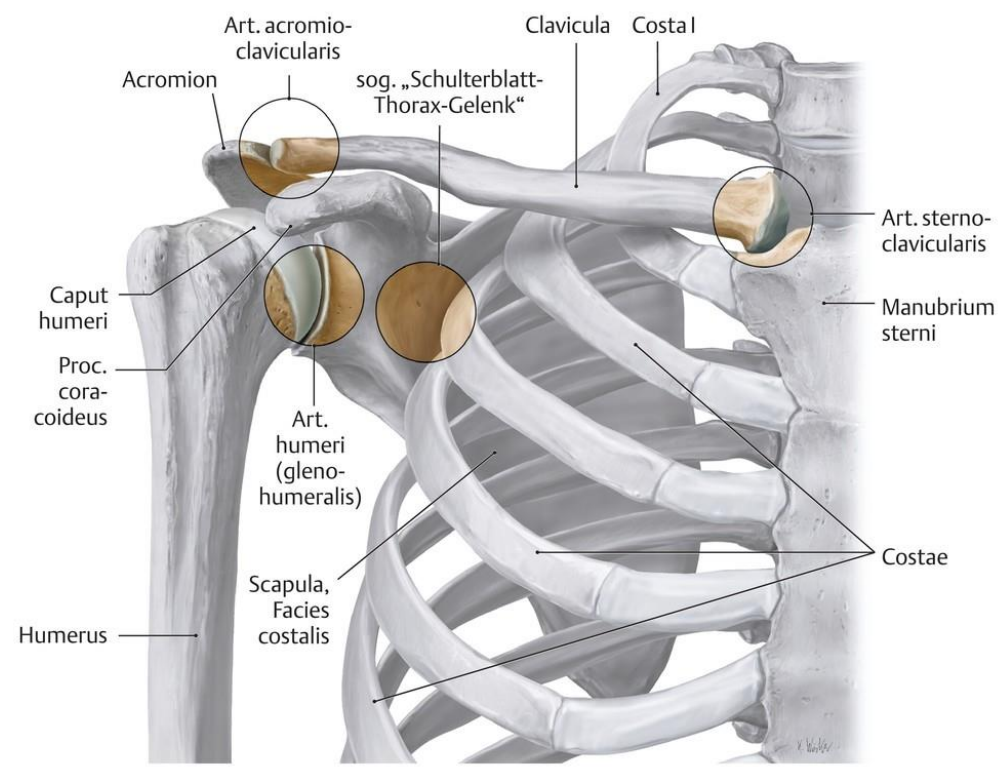

Abbildung 4: Gelenke der Schulter im Überblick[56]

Infolge der ungenügenden Knochenführung des Schultergelenks und einer, besonders im dorsalen Bereich, wenig ausgeprägten Sicherung des Gelenks durch den Bandapparat ist das Schultergelenk ein überwiegend muskelgesichertes Gelenk.[56, 57] Die sogenannte Rotatorenmanschette besteht aus dem M. supraspinatus (kranial), M. infraspinatus (dorsal), M. teres minor (dorsal) und M. subscapularis (ventral), die alle von verschiedenen Ursprüngen der Scapula zum Humerus ziehen. Entsprechend ihres Verlaufs können sie eine Abduktion oder Adduktion sowie eine Innen- oder Außenrotation bewirken.[56-59] Die große Beweglichkeit der oberen Extremitäten wird dabei durch eine Vielzahl von Muskeln ermöglicht (Abb. 5). Rumpf- und Schultergürtelmuskulatur lässt sich dabei nach ihrer Topografie in ventrale und dorsale Gruppen sowie nach ihrem Ansatz mit Ansatz am Humerus und Ansatz am Schultergürtel unterscheiden.[57] Bei der Schultergürtelmuskulatur mit Ansatz am Humerus lassen sich neben der genannten Rotatorenmanschette auch noch der M. deltoideus, M. teres major und M. latissimus dorsi nennen. Der M. deltoideus sitzt dem Schultergelenk kappenartig auf und umfasst es komplett. Entsprechend seiner Muskelanteile übernimmt er verschiedene Aufgaben, ist aber ein entscheidender Muskel für die Armabduktion. Der M. teres major entspringt dem Angulus inferior der Scapula und ist mit seinem Ansatz am Humerus für eine Armadduktion, Innenrotation und Retroversion verantwortlich.[57] Der M. latissimus dorsi gehört zur nicht-autochthonen Rückenmuskulatur. Er hat verschieden Anteile die von den Processi spinosi der Wirbelsäule, vom Beckenkamm sowie von den Rippenbögen und der Scapula entspringen. Somit überzieht er mit seinen Muskelfasern fast den gesamten Rücken.[57] 
Genauso wie der M. teres major setzt er am Humerus an und sorgt für eine Armadduktion, Innenrotation und Retroversion.[56-59]

Ein ventraler Schultermuskel mit Ansatz am Humerus ist der M. pectoralis major. Dieser entspringt mit seinen unterschiedlichen Anteilen von der Calvicula, dem Sternum und der Rektussscheide. Entsprechend seines Verlaufs führt er bei Kontraktion zu einer Adduktion, Innenrotation und Anteversion des Arms.[56, 57, 59]

Neben dem M. latissimus dorsi haben von den nicht-autochthonen Rückenmuskeln auch die Mm. rhomboidei, der M. levator scapulae, der M. serratus anterior und der M. trapezius Einfluss auf die Mechanik der oberen Extremitäten, allerdings mit Ansatz am Schultergürtel.[57, 58] Die Mm. rhombiodei ziehen von den Dornfortsätzen der Brust- bzw. Halswirbel mit der Aufgabe die Scapula in ihrer Position zu fixieren. Der M. levator scapulae entspringt von den Querfortsätzen des 1. bis 4. Halswirbels und zieht zum Angulus superior der Scapula. Er bewirkt bei Kontraktion somit ein Anheben des Schulterblatts. Der M. serratus anterior entspringt mit seinen Anteilen von der 1. bis 9. Rippe und zieht von hier zu unterschiedlichen Ansätzen an der Scapula. Er bewegt so die Scapula nach vorne und dreht sie nach außen, wodurch Armelevation und Anteversion möglich werden.[57, 58] Der M. trapezius entspringt am Hinterhauptbein (Protuberantia occipitalis externa) sowie an den Dornfortsätzen der Hals- und Brustwirbel und setzt am lateralen Teil der Clavicula sowie am Acromion und an der Scapula dorsal an. Bei Kontraktion kann er das Schulterblatt heben, nach dorsal ziehen und senken. Er kann außerdem die Clavicula nach dorsal ziehen. Somit unterstützt er bei der Armelevation. Bei unilateraler Kontraktion führt er zu einer Lateralflexion bei bilateraler Kontraktion zu einer Dorsalextension des Halses.[56-59] 


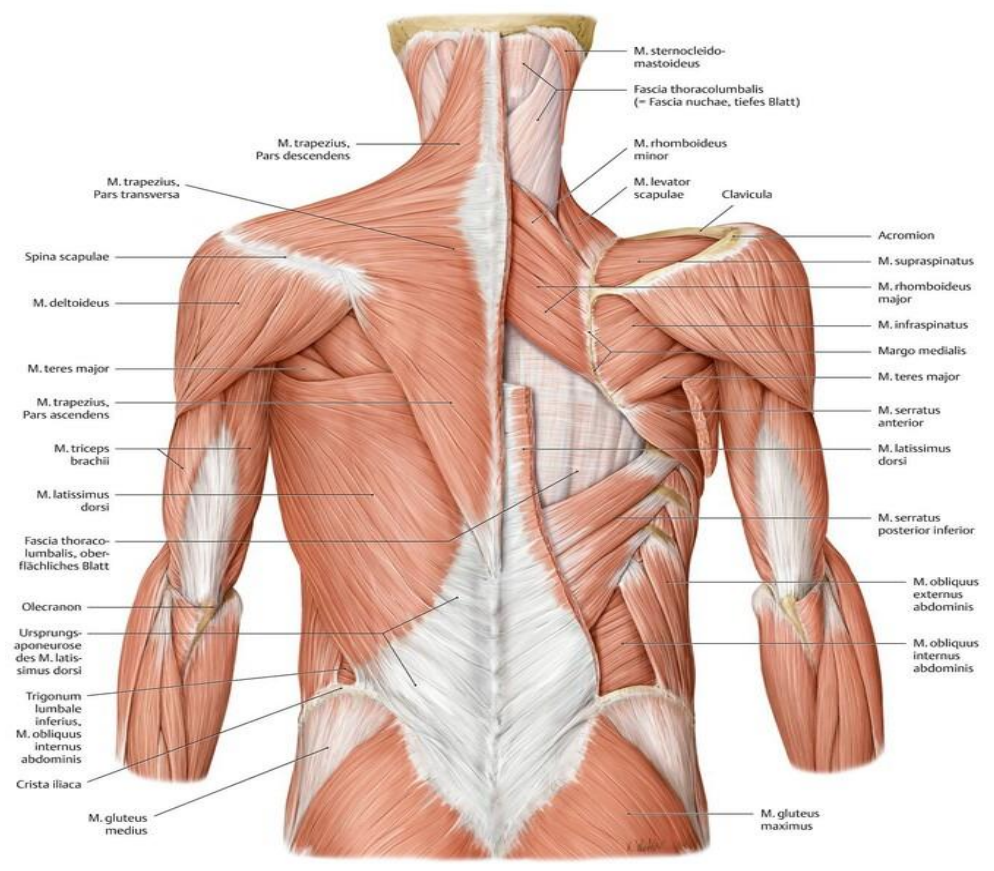

Abbildung 5: Muskulatur des Schultergürtels[58]

\subsection{Gesundheitliche Beeinträchtigungen des Stütz- und Bewegungsapparats in Form von Muskuloskelettalen Erkrankungen}

Der Begriff Muskuloskelettale Erkrankungen (MSE) beschreibt über 150 Krankheitsdiagnosen, die den Bewegungsapparat betreffen.[60] Er beinhaltet ein breites Spektrum an inflammatorischen sowie degenerativen Erkrankungen, das Knochen, Muskeln, Sehnen, Bänder, Gelenke, periphere Nerven und unterstützende Blutgefäße abdeckt.[60-63] Muskuloskelettale Erkrankungen beinhalten plötzlich auftretende, kurzlebige Konditionen, bis hin zu lebenslangen Konditionen assoziiert mit kontinuierlichen Beschwerden.[60] Typischerweise sind die Erkrankungen charakterisiert durch oftmals anhaltende Schmerzen und Mobilitätseinschränkungen sowie einer verminderten Geschicklichkeit und Funktionsfähigkeit. Diese können zu einer verminderten Funktionsfähigkeit und somit Einschränkung des Betroffenen im Sozial-, sowie Arbeitsumfeld führen.[60, 62, 64] Sind MSE primär durch die Arbeit oder die direkten Einflüsse des Arbeitsumfelds verursacht, wird von arbeitsbedingten MSE gesprochen.[62]

Muskuloskelettale Erkrankungen beinhalten konkrete Konditionen der Gelenke, wie z.B. Bandscheibenprolaps, Gicht, Osteoarthritis oder rheumatoide Arthritis. Sie beinhalten Konditionen der Knochen wie z.B. traumatische Frakturen, Osteoporose oder Osteopenie. Sie beinhalten ebenso Konditionen der Muskulatur, wie z.B. Myalgie (Muskelschmerz) 
oder Sakropenie. Außerdem beinhalten sie Entzündungen der Sehnen und Sehnenscheiden (Tenosynovitis) und verwandten Strukturen, z.B. Epicondylitis oder Bursitis. Ebenso beinhalten sie Konditionen der Nerven in Form von Funktionsstörungen und Kompressionen, wie z.B. Ischialgie, Carpaltunnel-Syndrom oder Dysästhesien. Der Begriff „Muskuloskelettale Erkrankungen“ beinhaltet aber auch unspezifische Konditionen multipler Bereiche oder Körperstrukturen, wie Rückenschmerzen und andere regionale Schmerzsymptome.[60, 61, 63, 64] Der Begriff umfasst folglich eine Vielzahl unterschiedlicher Krankheitsbilder des Halte- und Bewegungsapparats und findet deshalb oftmals Anwendung bei demographischen Analysen des Gesundheitszustands der Bevölkerung.

Weltweit sind mehrere Hundertmillionen Menschen von MSE betroffen.[64] Sie sind dabei die global führende Ursache für chronische Schmerzen, körperliche Funktionseinschränkungen und ein Verlust an Lebensqualität.[63] Dabei sind MSE die Hauptursache für Behinderung bzw. Arbeitsunfähigkeit in vier von sechs WHO Regionen (Platz Zwei in Region Afrika und Mittlerer Osten). Während die Prävalenz einzelner MSEs mit Art der Erkrankung und Bevölkerungsalter variiert, leben weltweit 20\% bis 33\% der Bevölkerung mit einer schmerzhaften muskuloskelettalen Kondition.[60, 65] In Europa geben drei von fünf Arbeitern muskuloskelettale Beschwerden an.[62] Obwohl die Prävalenz muskuloskelettalen Kondition mit dem Alter steigt, sind auch junge Menschen betroffen, oftmals während des Höhepunkts ihrer beruflichen und finanziellen Jahre.[60]

\subsection{Berufsbild von Zahnarzt und Zahnmedizinischen Fachangestellten unter beson- derer Berücksichtigung gesundheitlicher Risikofaktoren}

Der folgende Abschnitt beschreibt das Berufsbild von Zahnärzten (ZÄ) und Zahnmedizinischen Fachangestellten (ZFAs). Dabei soll besonders auf das Arbeitsumfeld sowie die Arbeitsbelastung und dessen Auswirkung auf die Gesundheit eingegangen werden. Arbeitsabläufe und physikalische Risikofaktoren, welche zu gesundheitliche Schäden des Halte- und Bewegungsapparats führen, werden erläutert.

Der Schwerpunkt des Tätigkeitsfelds von Zahnärzten und Zahnmedizinischen Fachangestellten besteht aus Prävention, Diagnose und Therapie von Zahn-, Mund- und Kiefererkrankungen. Dabei müssen sie sich in Ihrem Arbeitsalltag zuallererst nach dem mitgebrachten Arbeitsfeld ihrer Patienten richten. Der Patientenmund ist klein, eng und erfor- 
dert somit eine sehr hohe Arbeitspräzision.[2, 3] Die Mundöffnung des Patienten erschwert dabei die Sicht- und Lichtverhältnisse bei der Arbeit.[4] Aufgrund des eigenen Speichelflusses der Patienten sowie der verwendeten Wasserkühlung und Spülungen während der Arbeit ist ein Absaugen der Flüssigkeiten wichtig, um Arbeitsqualität und übersicht zu gewährleisten. Dabei sind die stark begrenzten Platzverhältnisse im Patientenmund stets zu beachten.[3,5]

Diese Gegebenheiten stellen sehr hohe physische Anforderungen an die Berufsgruppe.[3, 6-8] Das kleine Arbeitsfeld schränkt dabei oftmals stark den Bewegungsumfang ein, in dem während einer Behandlung gearbeitet wird. Infolge kommt es oft zu unangenehmen, gesundheitlich ungeeigneten Haltungen und Zwangshaltungen. Statische Haltungen, welche oft über lange Zeiträume während einer Behandlung oder eines Arbeitstages gehalten werden müssen, sind ein in der Literatur viel beschriebener Risikofaktor.[3, 4, 6, 10-12, 28] Morse [12] beschrieb in einer Studie, in der Zahnmedizinische Fachangestellte selbst biomechanische Risikofaktoren ihres Berufsfeldes aufzeigen sollten, dass erfahrene ZFAs zu über $60 \%$ statische Körperhaltungen als Gesundheitsrisiko angegeben hatten. Der Großteil dieser $60 \%$ gab des Weiteren an, statische Haltungen sehr oft einzunehmen. Die wenig existierende Literatur [3, 66] beschreibt, dass während der Behandlung mit einer hohen Frequenz statische Haltungen eingenommen werden, die eine Kontraktion von über 50\% der Körpermuskulatur erfordern, um den Körper bewegungslos zu halten während er der Erdanziehungskraft resistiert. Die statischen Kräfte, welche aus diesen Haltungen resultieren, können zu Muskelermüdung und einem gestörten Gleichgewicht innerhalb der Muskulatur führen.[3, 4, 12, 29] Bei unkorrigierter Haltung können die physiologischen Konsequenzen wiederholter, prolongierte Phasen statischer Belastung zu Muskelischämie/-nekrose, Schmerzen (Myalgie) sowie zu abnormaler Haltung führen. Später können sie dann in Gelenkhypomobilität, Bandscheibendegeneration und weiteren Muskel-Skelett-Erkrankungen resultieren.[3]

Während der Behandlung ist, aufgrund der räumlichen Einschränkungen der Behandlungseinheiten und der damit verbundenen Positionierung- bzw. Lagerungsmöglichkeiten von Behandler ${ }^{2}$ und Patient, eine gute Einsicht in die Mundhöhle nur nicht immer möglich. Da das Arbeitsfeld zumeist unterhalb der Augenhöhe der Behandler liegt resultiert

\footnotetext{
${ }^{2}$ Als Behandler werden im Folgenden sowohl Zahnärzte als auch Zahnmedizinische Fachangestellte verstanden. Sie arbeiten gemeinsam für das Wohl des Patienten und fungieren während der Behandlung als Team.
} 
in der Praxis deshalb oft eine krumme, vorgebeugte Haltung mit runden Schultern (Abb. 6).[3, 4, 12, 13] Eine Studie, in der 204 Zahnärzte und ZFAs beim Praktizieren ihrer Arbeit beobachtet wurden, konnte bei $83 \%$ eine vorgebeugte Haltung feststellen.[14] Marklin und Cherney [67] beschrieben in einer Studie die die Arbeitshaltung von Z ̈̈ sowie ZFAs untersuchte, das beide Professionen 86\% ihrer Arbeitszeit mit einem mindestens um $30^{\circ}$ vorgebeugten Nacken verbrachten. 50\% der Arbeitszeit bei den ZFAs und 53\% bei den ZÄ wurden mit mindestens $30^{\circ}$ vorgebeugtem Rumpf verbracht. Eine vermehrte und prolongierte Einnahme solcher Haltung kann, wiederum zu einem Ungleichgewicht der betroffenen Muskulatur führen. Es kann zur Schwächung und Elongation der stabilisierenden Haltemuskulatur der Schulterblätter kommen, in diesem Fall des M. trapezius, M. rhomboideus, M. serratus anterior.[3] Außerdem erhöht die Haltung die Kräfte auf die obere Nackenmuskulatur (M. trapezius, M. levator scapulae,), die Mm. Scaleni sowie auf die Bandscheiben.[13, 68] Die Folge können Verspannung, sowie Krämpfe der betroffenen Muskulatur, in schweren Fällen auch „Tension Neck Syndrom“ oder Engpasssyndrom der oberen Thoraxaperatur (Thoracic-outlet-Syndrom) sein.[32]

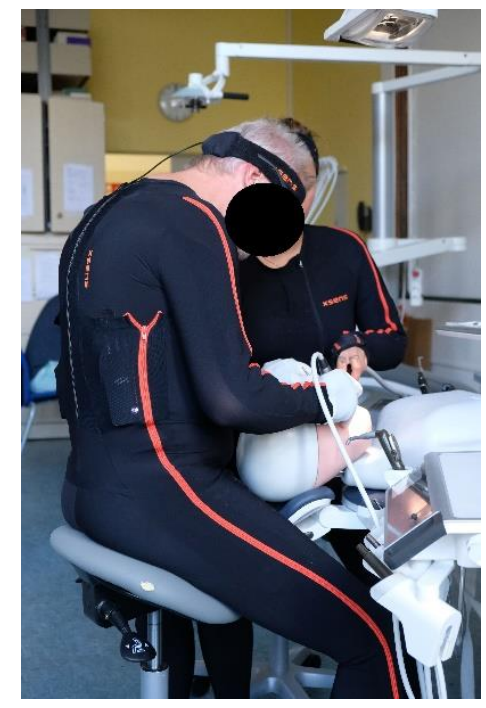

Abbildung 6: Vorgebeugter Nacken während Patientenbehandlung

Aufgrund der hohen Präzisionsanforderungen reicht Vorbeugen oftmals nicht aus, besonders wenn eine direkte Visualisierung erzielt werden soll. Für eine bessere Sicht kommt es deshalb oft zu wiederholter Rotation von Kopf, Nacken und Rumpf zu einer Seite, was in einer unangenehmen Haltung für der Behandler resultiert.[3, 4, 10, 12, 69] Morse [12] beschreibt in seiner bereits angeführten Studie, dass über 70\% der erfahrenen ZFAs Nackenrotation und über 90\% Nackenbeugen als beruflichen Risikofaktor ansehen. Eine schwedische Studie, an der randomisiert 143 schwedische Zahnarztpraxen teilnahmen, 
zeigte, dass Behandler mit cervicobrachialen Beschwerden ihren Kopf stärker vorbeugten und seitwärts rotierten als Behandler ohne Beschwerden.[70] Wie mehrere Autoren beschreiben $[3,12,29]$, wird dann die für die Rotation zuständige Muskulatur stärker und kürzer während die antagonistischen Muskeln schwächer werden und elongieren. Neben Schmerzen und Muskelischämie kann es infolge der asymmetrischen Kräfte auf die Wirbelsäule zur Falschausrichtung und einem verringerten Bewegungsumfang kommen. Rundcrantz et al. [71] zeigten, dass für einen Großteil der Zahnärzte die Bewegung einer Nackenrotation nach rechts mit gleichzeitigem Beugen nach links schwierig durchzuführen ist. Aufgrund der Tatsache, dass i.d.R. rechtshändige Z̈̈, um eine bessere Sicht zu erreichen genau die gegenteilige Haltung einnehmen. Sie arbeiten dann mit rotiertem Nacken nach links und einer Beugung nach rechts. Das Resultat sind über zumeist lange Zeiträume eingenommene Zwangshaltungen ${ }^{3}$, mit isometrischer Muskelkontraktion [4], bei denen es außerdem zur Torsion, Flexion, Lateralfelxion des Rumpfes kommt und es infolge wiederum zu einer Störung des muskulären Gleichgewichts kommen kann.[10] Im Rahmen einer Behandlung muss ein Behandler oft, z.B. zum Halten von Instrumenten, über lange Zeiträume mit angehobenen und abduzierten Armen arbeiten.[7] Beispielsweise zum Halten eines Spiegels, um das Arbeitsfeld indirekt zu visualisieren oder Anatomie der Mundhöhle aus dem Arbeitsfeld abzuhalten.[12] Eine Unterbrechung oder Entspannung dieser Haltung ist meist erst bei Abschluss der Behandlung möglich. Dabei konnte gezeigt werden, dass die statische Arbeitsbelastung, insbesondere des M. Trapezius, in enger Relation zu Schulterproblemen stehen.[7, 15] Finsen [7] untersuchte in einer Laborstudie an Z $\overparen{A}$ die drei üblichsten Arbeitsaufgaben, die Arbeitshaltung und Muskelbelastung mittels Elektromyographie. Dabei beschrieb er eine hohe statische Arbeitsbelastung der Nacken- und Schulterregion. Außerdem fand eine Armabduktion über $30^{\circ}$ während ungefähr 1/3 der Zeit statt. Lange Perioden mit erhobenem Arm können dabei ein erhöhtes Risiko für Schmerzen im M. Trapezius bedeuten.[3] Eine generelle, nicht nur auf zahnmedizinische Fachkräfte beschränkte, systematischen Übersichtsarbeit [12] fand dabei einen gut dokumentierten Zusammenhang zwischen Problemen in der Schulterregion und hohen physischen Arbeitsbelastungen. Bei unangenehmen und statischen Arbeitshaltungen und/oder exzentrischer Belastung bestand dieser Zusammenhang auch

\footnotetext{
${ }^{3}$ Zwangshaltungen liegen vor bei von der Ruheposition im Stehen oder Sitzen deutlich abweichenden Körperhaltungen mit geringen Bewegungsmöglichkeiten, die über längere Zeiträume ohne Unterbrechung oder Belastungswechsel eingenommen werden. Es ergibt sich eine erhöhte körperliche Belastung durch ermüdende statische Muskelspannung .[72]
} 
schon bei niedrigem externen Gewicht.[12, 16] In Griechenland befragte eine Studie [18] $430 \mathrm{ZÄ} \mathrm{zu} \mathrm{selbst} \mathrm{ausgemachten} \mathrm{physischen} \mathrm{Arbeitsbelastungen} \mathrm{und} \mathrm{der} \mathrm{Prävalenz} \mathrm{von}$ muskuloskelettaler Beschwerden. Dabei erhöhte die Angabe von unangenehmen Arbeitshaltungen, anstrengender Armhaltung, Armabduktion, Armanhebung, Gebrauch vibrierender Instrumente und repetitiven Bewegungen das Risiko muskuloskelettaler Beschwerden in der Schulter um den Faktor 2,6.

In vielen Fällen diktiert das Behandlungsprozedere einen stark repetitiven, monotonen Bewegungsablauf, was vielfach als Risikofaktor für muskuloskelettale Beschwerden beschrieben wurde.[4, 11, 12, 17-19, 73] Beispielsweise wird während einer Wurzelkanalbehandlung, einer eher langwierigen Behandlung, immer wieder mit Pfeilen und anderen Instrumenten unterschiedlicher Größe das gleiche Bewegungsmuster durchgeführt bis der Wurzelkanal ausreichend aufbereitet ist. Unter erfahrenen ZFAs geben in einer US-amerikanischen Studie dabei über 95\% repetitive Bewegungen als gesundheitlichen Risikofaktor an.[12] Die Einnahme statischer Körperhaltung und Aufwendung von Kraft für den Gebrauch der Dentalinstrumente, wie z.B. beim Entfernen von Zahnstein (Scaling) oder oralchirurgischen Operationen üblich, kann die Arbeitsbelastung dabei nochmal deutlich erhöhen.[4, 17, 19] Eine Vergleichsstudie, die die Prävalenz und Risikofaktoren für Handprobleme unter ZFAs untersuchte, konnte zeigen, dass Behandler von Patienten mit ausgeprägtem Zahnstein und somit höherer Arbeitsbelastung eine um den Faktor 2,3 höhere Wahrscheinlichkeit hatten Handprobleme zu entwickeln als ihre Vergleichsgruppe mit wenig Zahnstein. Auch zeigte die Studie das ZFAs mit mehr als zehn Jahren Arbeitserfahrung eine um den Faktor 1,9 höhere Wahrscheinlichkeit hatten Symptome assoziiert mit dem „Carpal-Tunnel-Syndrom“ zu manifestieren.[17] Warren [19] beschreibt in den Ergebnissen einer Studie, in der 160 ZFAs befragt und untersucht wurden, dass diagnostizierte MSE primär assoziiert waren mit repetitiver Arbeit der Schultern, Arme und Handgelenke, sowie Arbeiten mit vibrierenden Instrumenten. Außerdem beschreibt Hayes [73] in einer deskriptiven Studie, dass die Art des Scalings einen Einfluss auf potenzielle MSE hat. Die Studie zeigte, dass ZFAs, die mit Handinstrumenten arbeiteten, eher Nackenbeschwerden entwickeln während ZFAs, die mit vibrierenden Instrumenten arbeiten, eher Schulterbeschwerden und Beschwerden des oberen und unteren Rückens angegeben hatten. Die Verwendung vibrierender Instrumente gehört sowohl für Zahnärzte (zum Beschleifen und Aufbereiten von Zähnen) als auch für ZFAs (zum Entfernen von Zahnstein) zum alltäglichen Gebrauch in fast jeder Behandlung. Alexopoulos 
[18] beschreibt dabei den Zusammenhang, dass Zahnärzte, die vibrierende Instrumente selbst als Arbeitsbelastung ausmachen, 2,9 mal öfter Schulterbeschwerden angaben als Kollegen, die vibrierende Instrumente nicht angegeben hatten. In einer klinischen Studie [33], wurden 94 erfahrene ZFAs, welche regelmäßig hochfrequente Ultraschallscaler benutzten, auf ihre periphere Nervenfunktion, klinische Anzeichen und Symptome untersucht. Chronische Parästhesien der Hand wurden dabei bei 44,7\% der Probanden beschrieben. Probanden mit Carpal-Tunnel-Syndrom verwendeten dabei vibrierende Geräte wöchentlich fast doppelt so viel wie Probanden ohne die Erkrankung und hatten eine erniedrigte Nervenleitungsgeschwindigkeit.

\subsection{Prävalenz muskuloskelettaler Konditionen unter Zahnärzten und Zahnmedizi- nischen Fachangestellten}

Die Prävalenz von muskuloskelettalen Beschwerden unter Zahnärzten und ZFAs wurde von zahlreichen internationalen Studien [4, 6, 10, 12, 14, 17, 18, 26, 27, 29-32, 74-77] untersucht. Diese zeigen dabei weltweit eine hohe Prävalenz. Lietz et al. [6] beschreiben in einer Metaanalyse von 41 Studien, welche die Prävalenz unter ZÄ und ZFAs analysierten, eine 12-Monatsprävalenz zwischen 60,8 und 96,9\% der jeweiligen Probanden für MSE. Die aktuelle Prävalenz lag bei den untersuchten Studien zwischen 48,1 und 91,8\%. In einer umfragebasierten tschechischen Studie [30] mit 581 ZÄ gaben für die letzten zwölf Monate 96,9\% aller Probanden an, zumindest geringe muskuloskelettale Beschwerden gehabt zu haben, wobei 66,3\% moderate bis starke Beschwerden angaben. Dabei waren Nacken-, Schulterbeschwerden sowie Kopfschmerzen am häufigsten.[30] Leggat et al. [76] beschreiben unter australischen Zahnärzten eine 12-Monatsprävalenz mit zumindest einem MSE-Symptom von 87,2\%. Wobei mit 57,5\% Nacken-, 53,7\% unterer Rücken- und 53,3\% Schulterbeschwerden die Regionen mit den meisten Beschwerden waren. Des Weiteren gaben 37,5\% der Probanden an, in den letzten zwölf Monaten medizinische behandelt oder beraten worden zu sein. 9,1\% sagten aus, im letzten Jahr krankheitsbedingt ausgefallen zu sein, wobei die betroffenen Probanden im Schnitt 11,5 Tage krankgeschrieben waren.[76] Eine deutsche Studie [10] untersuchte die Prävalenz von MSE unter 406 ZFAs. Dabei gaben die 98,5\% der Probanden an, zumindest in einer Körperregion in ihrem Leben Beschwerden gehabt zu haben. Unter den Probanden gaben 97,5\% zumindest eine Beschwerde in den letzten zwölf Monaten und 86,9\% in den letzten 
sieben Tagen an. Für alle Prävalenzen war der Nacken die am öftesten betroffene Region, gefolgt von Schultern, oberem und unterem Rücken.[10] Unter serbischen Zahnärzten gaben $82,6 \%$ an aktuell unter muskuloskelettalen Beschwerden zu leiden, dabei waren der Nacken mit 49,5\% unter allen Probanden und der untere Rücken mit 46\% die meistbetroffenen Körperregionen. Des Weiteren sagten 22,8\% der Z̈̈ unter Symptomen des Carpal-Tunnel-Syndroms zu leiden. 49,3\% der Probanden gaben an die schlimmsten Schmerzen während der Arbeit zu spüren.[77] Bereits unter Zahnmedizinstudenten beschreiben Thornton et al. [27] in den USA, dass von 590 Probanden 61\% muskuloskelettale Beschwerden oder Schmerzen assoziiert mit ihrer Arbeit in der Zahnklinik angaben. Dabei waren $78 \%$ der Studenten mit Beschwerden unter 29 Jahre alt. Die meist affektierten Körperregionen waren der Nacken mit 48\%, der Rücken mit 44\%, die Schultern mit 31\% und die Hände mit 20\% unter allen Probanden mit Beschwerden.

In der bereits angeführten systematischen Übersichtsarbeit analysierten Lietz et al. [6] die in 23 Studien aufgeführten Prävalenzen nach Körperregion. Dabei waren der Nacken, gefolgt vom Rücken und den Schultern die drei meistaffektierten Körperregionen. Die 12-Monatsprävalenz lag jeweils für den Nacken zwischen $29,1 \%$ und $84,8 \%$. Für den Rücken befand sich die 12-Monatsprävalenz generell zwischen 26,7\% und 57,1\%, wobei die Prävalenz für den unteren Rücken mit 28,5\% und 74,9\% höher lag. In den Schultern lag die 12-Monatsprävalenz zwischen 6,1\% und 69,6\%. Die Werte in der Nackenregion für die 7-Tageprävalenz waren zwischen 19,7\% und 75\%, für den unteren Rücken zwischen 21 und 28,5\% und in der Schulterregion zwischen 16,7\% und 28,9\%. Die Metaanalyse zeigte dabei konsistente Resultate über alle Prävalenzperioden außer für die Punktprävalenz. Ebenfalls oft auf MSE-Prävalenz analysierte, affektierte Körperregionen für eine 12-Monatsprävalenz waren Hände/Handgelenke zwischen 16,7\% und 66,9\% der Probanden, Ellenbogen zwischen 5,5\% und 29,1\%, Arme zwischen 6,3\% und 53,3\%, Hüften $11,1 \%$ und $39,7 \%$, Knie zwischen $10,3 \%$ und 37,3\%, Beine zwischen 3,1\% und 35,1\% und Füße/Sprunggelenke zwischen 10\% und 17,6\%.[6] Morse [12] analysierte in einer systematischen Übersichtsarbeit die MSE Prävalenz von Nacken- und Schulterregion getrennt für Zahnärzte, ZFAs und Zahnmedizinstudenten. Die 12-Monatsprävalenz der Nackenregion lag dabei für Zahnärzte zwischen $17 \%$ und 73\%, für ZFAs zwischen $38 \%$ und $83 \%$. In der Schulterregion war die 12-Monatsprävalenz für Zahnärzte zwischen 
$20 \%$ und $65 \%$, für ZFAs zwischen $27 \%$ und $76 \%$. Für Studenten waren die 12-Moanatsprävalenzen in der Nackenregion zwischen 29 und 42\% und für die Schulterregion zwischen $1,7 \%$ und $20 \%$.[12, 27]

Die hohe Prävalenz von MSE unter ZÄ und ZFAs können dabei der Grund sein, warum Tätigkeiten im Arbeitsfeld nur unter Schmerzen ausgeführt werden können [29, 66, 78, 79] oder der Beruf gar nicht mehr ausgeübt werden kann.[80, 81] Brown [80] untersuchte dabei 189 Fälle von krankheitsbedingtem Ruhestand bei Zahnärzten, von denen 55\% MSE als Ursache ihres vorzeitigen Ruhestands angaben. Von den Probanden sagten 90\% aus, dass ihre schlechte Gesundheit arbeitsbedingt war.[80] Eine Studie [66] mit israelischen Zahnärzten untersuchte den Zusammenhang zwischen MSE und der Arbeitshaltung. Die 12-Monatsprävalenz für MSE im unteren Rücken und im Nacken lag bei $55 \%$ und 38,3\%. Dabei konnte eine signifikante Korrelation zwischen der im Sitzen verbrachten Arbeitszeit und der Ausprägung von Rückenbeschwerden festgestellt werden. Zudem gaben $8 \%$ der Probanden an, ihre Arbeit aufgrund der Beschwerden ihre Arbeit nicht normal durchführen zu können.[66] In einer kanadischen Studie [79], welche die ergonomischen Risikofaktoren in der klinischen Zahnmedizin untersuchten, gaben von $421 \mathrm{ZÄ}$ 19\% an aufgrund muskuloskelettaler Beschwerden nur eingeschränkt Freizeitaktivitäten ausführen zu können. Wiederum 14\% gaben an Aktivitäten im eigenen Haushalt nur eingeschränkt ausführen zu können.[79]

Die Arbeitsbelastung in Form von Arbeitsstunden und der Anzahl behandelter Patienten hat dabei einen signifikanten Einfluss auf die MSE Prävalenz [6, 7, 9, 12, 29, 30, 82-84]. Von 5120 befragten ZÄ und ZFAs der US-Army gab ein Großteil an, ungefähr 40 Stunden die Woche zu arbeiten, dabei behandelten sie 31 bis 50 Patienten pro Woche.[74] 406 Deutsche ZFAs gaben im Schnitt an, pro Woche 38,5 Stunden zu arbeiten, 30 davon am Patienten.[10] Eine schwedische Studie [84], die den Zusammenhang zwischen Arbeitsbedingungen und arbeitsbedingter Krankschreibug untersuchte, konnte dabei zeigen, dass ZFAs mit MSE dazu neigten pro Tag mehr Patienten zu behandeln und mehr Arbeitsstunden zu sammeln als ZFAs ohne MSE.[84] Dabei korreliert die Arbeitszeit am Patienten ebenfalls positiv mit der MS-Prävalenz.[83] Zarra et al. [82] untersuchten in einer griechischen Studie den Zusammenhang zwischen der Anzahl behandelter Patienten und dem Risiko für muskuloskelettale Beschwerden. Dabei stellten sie fest, dass ZÄ und ZFA, die 6 bis 8 Patienten pro Tag behandelten, ein um den Faktor 3,52 höheres Risiko hatten als 
die Vergleichsgruppe mit weniger als 6 Patienten pro Tag. Zum gleichen Resultat gelangte Hodacova [30] in einer tschechischen Studie mit 20 Patienten pro Tag.

\subsection{Ergonomie in der Zahnmedizin}

Der Begriff „Ergonomie“ leitet sich her aus dem Griechischen ergon (Arbeit) und nomos (Gesetze).[85] Die International Ergonomics Association (IEA) bezeichnet sie als die wissenschaftliche Disziplin, welche sich mit dem Verständnis der Interaktion zwischen Menschen und anderen Elementen eines Systems befasst sowie als die Profession, welche Entwicklungstheorie, -prinzipien, -daten und -methoden anwendet für die Optimierung der menschlichen Gesundheit und der allgemeinen Systemleistung.[86] Allgemein wird es als Aufgabe der Ergonomie betrachtet, den Beruf an den Arbeiter anzupassen.[87, 88] Laut der European Society of Dental Ergonomics ist die Ergonomie in der Zahnmedizin definiert, als Adaption des Arbeitsumfelds sowie der Arbeitsmethoden an den Zahnarzt und sein Team unter Berücksichtigung ihrer physischen und psychologischen Kapazitäten bezüglich einer gesunden, sicheren und komfortablen Funktion in ihrem Beruf.[89] In der Zahnmedizin hat die Lehre der Ergonomie somit die primäre Aufgabe präventiv muskuloskelettalen Beschwerden entgegen zu wirken [90], da die Prävention von MSE einfacher ist als ihre Behandlung [91] und auch behandelte MSE bei ähnlichen Arbeitsmustern wieder auftreten können.[3, 4, 92]

In der Zahnmedizin gibt es bezüglich der optimalen Arbeitshaltung und dem davon abgeleiteten Design von Behandlungsumfeld und Instrumenten keine wissenschaftlich einheitliche Linie[88]. Definierte ergonomische Standards wie DIN EN 1005-04[93] und ISO 11226[20], ISO 6385[94] können auch in der Zahnmedizin Anwendung finden. Meist abgeleitet von diesen ergonomischen Standards machen verschiedene Autoren [2124] Angaben zu einer optimalen Arbeitshaltung, allerdings ohne zugrundeliegende experimentelle Daten aus der Zahnmedizin. Im Folgenden wird eine, in Übereinstimmung diverser Autoren [21-25, 87, 95-99], ergonomisch optimale Arbeitshaltung für Z̈̈ und ZFAs beschrieben: Der Oberkörper sollte, falls notwendig, vom Hüftgelenk aus maximal $10^{\circ}$ bis $20^{\circ}$ vorgebeugt sein.[25, 99] Grundsätzlich wird angenommen, dass je stärker ein Gelenkwinkel von seiner entspannten Haltung abweicht, desto größer das Verletzungsrisiko.[97] Es wird beschrieben, dass ein Beugen des Oberkörpers und Kopfs seitwärts vermieden sowie dass der Kopf maximal $20^{\circ}$ vorgebeugt werden soll. Nach Einnahme der 
Arbeitshaltung sollte der Abstand zwischen den Augen des Behandlers und dem Patientenmund als Arbeitsfeld zwischen 35 und $45 \mathrm{~cm}$ betragen. Die Arme sollen dem Oberkörper anliegen, um Bewegungen des Oberarms soweit wie möglich zu minimieren, mit zum Boden parallel ausgerichteten Schultern. Die Oberarme sollen bei Seitwärtsbewegungen maximal bis 15 bis $20^{\circ}$ und Vorwärtsbewegungen maximal $25^{\circ}$ nicht überschreiten. Die Unterarme sollten parallel zum Boden gehalten werden, dabei sollte die Flexion im Winkel zum Oberarm zwischen 70 bis $100^{\circ}$ liegen. Die Handgelenke sollen idealerweise entspannt gehalten werden, mit einer maximalen Flexion/Extension von $15^{\circ}$. Eine Rotation soll vermieden werden. Der Winkel im Hüftgelenk zwischen Rumpf und Beinen sollte über $90^{\circ}$ sein, um eine Rotation des Beckens zu vermeiden, und zwischen $105^{\circ}$ und $125^{\circ}$ liegen. Der Winkel zwischen Ober und Unterschenkel sollte bei ungefähr $110^{\circ}$ liegen und beide Füßen sollen sicher abgestützt sein.[21-23, 25, 87, 95, 96, 98, 99]

Das Einnehmen und Einhalten dieser idealen ergonomischen Arbeitshaltung, durch die Behandler, wird in der Zahnmedizin auf unterschiedlichen Wegen versucht zu erreichen. Grundsätzlich werden verhaltenspräventive und verhältnispräventive Maßnahmen unterschieden. Diese setzen entweder am individuellen Verhalten des Arbeiters (verhaltenspräventiv) oder an den Arbeitsverhältnissen des Arbeiters (verhältnispräventiv) an.[100] Verhaltenspräventive Maßnahmen sind z.B. im Laufe der Ausbildung stattfindende oder postgraduale Ergonomieschulungen und -lehrveranstaltungen. Den Teilnehmern werden hierbei Arbeitstechniken und Arbeitshilfen vermittelt, welche ihnen ein ergonomisches Arbeiten ermöglichen soll. Das Lehrprogramm und die Gewichtung der einzelnen Themenfelder entsprechen dabei der Lehrmeinung des Unterrichtenden. Grundsätzlich werden die Teilnehmer darin geschult wie sie ihr Equipment am besten nutzen. Den Teilnehmern wird beigebracht wie sie ihre Behandlungsstühle einzustellen und zu modifizieren haben, um eine optimale Körperhaltung und Unterstützung der Haltemuskulatur zu erzielen. Des Weiteren werden sie geschult wie sie sich in Relation zum Patient positionieren müssen und wie der Patientenstuhl sowie die Arbeitsleuchte eingestellt werden müssen für eine ergonomische Arbeitsweise bei maximaler Einsicht und Zugänglichkeit ins Arbeitsfeld. Auch das optimale Lagern der Patienten auf dem Stuhl wird vermittelt.[21, 22, 24, 101] Zudem beschreiben manche Autoren [24, 101, 102], wie z.B. erstmals Dr. Daryl Beach mit seinem Konzept der ,propriozeptiven Derivation“ $[101,102]$ und später Valachi [24], Techniken der Selbstwahrnehmung, die Behandlern helfen sollen ungesunde Arbeitshaltung frühzeitig selbst zu erkennen und zu korrigieren. 
Dabei herrscht keine Einigkeit darüber wie oft der Behandler seine Haltung während der Behandlung verändern soll. Während einige manche Universitäten und Lehrprogramme die Lehrmeinung einer einzigen Grundhaltung (,,home position“) vertreten, wird andererseits die Notwendigkeit multipler Grundhaltungen propagiert mit dem Ziel möglichst viele Muskelgruppen zu aktivieren, um die Arbeitsbelastung zu verteilen.[24, 39, 103, 104] Auch Techniken der Zusammenarbeit zwischen ZÄ und ZFAs, wie das vierhändige Arbeiten, die auf organisatorischer Ebene eine ergonomische Arbeitsweise ermöglichen, sind Teil der Eronomielehre.[105] Obwohl der Großteil dieser verhaltenspräventiven Maßnahmen bereits lange bekannt sind, gibt es bis jetzt nur verhältnismäßig wenige Studien, die eine Reduktion der MSE Prävalenz und Verbesserung der Arbeitshaltung in der Zahnmedizin zeigen.[4, 79, 101, 106-108] Insbesondere der Mangel klinischer Studien, die die Effektivität von Ergonomietraining und Ergonomieschulungen in der Zahnmedizin untersuchen wird deutlich.

$\mathrm{Zu}$ den verhältnispräventiven Maßnahmen zählen ergonomisches Equipment und Arbeitsplatzgestaltung, welche eine korrekte, gesunde Arbeitshaltung und gleichzeitig maximale Zugänglichkeit und Sicht ermöglichen sowie die Arbeitsbelastung verringern. Die „Occupational Safety and Health Administration“(OSHA) beschreibt technische und designtechnische Verbesserungen des Arbeitsplatzes als das schnellste und effektivste Mittel gegen ergonomische Gefahren.[109] Dabei ermöglicht, z.B. die Verwendung von Lupenbrillen und OP-Mikroskopen, dem Behandler eine verbesserte Ausleuchtung sowie detailreichere Darstellung des Arbeitsfeldes ohne die Notwendigkeit den Behandler-Patienten Abstand zu verringern im Sinne eines Verbeugens, Rotierens und letztlich Einnehmens ungeeigneter Arbeitshaltungen.[34, 35, 110, 111] Die ISO Standard 6385 beschreibt die ergonomischen Prinzipien im Design von Arbeitssystemen, auf dessen Grundlage die Gestaltung dentalem Equipments erfolgen soll.[94] Korrekt gestaltete Behandlerstühle ermöglichen dem Becken eine neutrale Position einzunehmen und verringern so eine Überbelastung der Wirbelsäule. Studien [24, 34, 35, 66, 112] zeigten, dass z.B. die Verwendung von Sattel-Stühlen dem Behandler ein schmerzfreies, ergonomisches Arbeiten ermöglicht. Beim Instrumentendesign konnte, z.B. im Fall von Küretten, gezeigt werden, dass leichtere Instrumente mit einem größeren Durchmesser die muskuläre Belastung der Hand verringern können. Das Resultat sind eine geringere Schmerzprävalenz der betroffenen Hand-, Unterarm- und Schulterregionen sowie ein erhöhter Be- 
handlungskomfort für den Behandler.[36, 37, 51] Die hier exemplarisch angeführten ergonomischen Anpassungen sind dabei nur ein Bruchteil der Möglichkeiten in der heutigen Zahnmedizin.

Außer Ergonomieschulungen und ergonomischen Equipment werden des Weiteren auch die Vorteile regelmäßiger Entlastungspausen sowie regelmäßigen Stretchings beschrieben.[24, 74, 113] Diese dienen als direkte Ausgleichübungen der Prävention von MSE, wobei verschiedene Übungen beschrieben sind, die während oder zwischen den Behandlungen von ZÄ und ZFAs ausgeführt werden können.[24, 108, 114] Lehto [39] beschrieb bereits 1991 den Zusammenhang zwischen besserer genereller physischer Fitness und besserer muskuloskelettaler Gesundheit. In einer systematischen Übersichtsarbeit [40] zum Thema Ergonomische Risiken und ihre Präventiven Maßnahmen in der Zahnmedizin wurden 29 relevante Studien untersucht. Als zentrale Präventivmaßnahme wurde modernes ergonomisches Instrumentarium in $61,5 \%$ der Studien angeführt, gefolgt von Strechingübungen in 53,8\%, dem Einhalten eines gesunden Lebensstils, einer korrekten ergonomischen Arbeitshaltung in 46\% und dem Einhalten kurzer Entlastungspausen sowie adäquater Arbeitsplatzausstattung bezüglich Belichtung und Lupenbrillen in 30,8\% der Studien. Weniger Beachtung findet das Abwechseln sitzender und stehender Arbeitshaltung sowie die Unterstützung durch eine Assistenz.[40] Unter präventiven ergonomischen Maßnahmen ist also die Wichtigkeit von körperlicher Aktivität und einer neutralen und ausgeglichenen Haltung weitgehend anerkannt. Trotzdem finden sich in der zahnmedizinischen Literatur [38, 53] nur vereinzelt und noch weniger qualitativ hochwertige Studien, die beschreiben welche Art von physischem Training adäquat ist zur Prävention von MSE.

Der Großteil der Informationen über eine gesunde, ergonomische Arbeitshaltung und die sich daraus abzuleitenden Paradigma der Ergonomie kursieren bereits seit über 20 Jahren.[21, 25, 87, 95, 96, 99] Trotz der gut belegten technischen, gestalterischen und organisatorischen Fortschritte in der Ergonomie, um den Beruf an ZÄ und ZFAs anzupassen, ist die Prävalenz Muskuloskelettaler Erkrankungen und Beschwerden nach wie vor in dieser Berufsgruppe sehr hoch. Das suggeriert, dass die Begebenheiten des Berufsfeldes von ZÄ und ZFAs nur eine eingeschränkte Effektivität verhältnispräventiver ergonomischer Interventionen zulassen.[7, 38] Außerdem wird eine wissenschaftliche Lücke über die Effektivität verhaltenspräventiver Maßnahmen, insbesondere dem Stellenwert körperlicher Fitness, in der Zahnmedizin deutlich. 


\subsection{Ergonomische Risikobeurteilung}

Die Ergonomie und die ergonomischen Risiken eines Arbeitsplatzes lassen sich anhand so genannter ,ergonomic risk assesment tools“ (ERAT) evaluieren.[115-117] Dabei lassen sich die zu evaluierenden Risiken nach Winkel und Mathiassen [118] in sechs Dimensionen unterscheiden. Erstens Kräfte, welche auf den Arbeitnehmer während Verrichtung der Arbeit wirken und solche, die er selbst aufbringt. Zweitens die Häufigkeit, mit der solche Kräfte wirken oder aufgebracht werden. Drittens die Dauer der körperlichen Arbeit. Viertens die Art der Arbeitshaltung und ihrer Variabilität, unterschieden in dynamische Bewegung und statische Haltung. Fünftens Vibrationen, die auf den Arbeitnehmer am Arbeitsplatz einwirken und sechstens psychologische Arbeitsbelastung.[119] Je nach Dimension der zu evaluierenden Risiken muss der Untersucher sich zwischen drei grundsätzlichen Werkzeugen entscheiden, die jeweils Vor- und Nachteile haben. Es wird dabei in Eigenangaben, observatorische Methoden und direkte Messungen differenziert. Eigenangaben werden beispielsweise erhoben über Fragebögen, Skalen oder Interviews, in denen die Probanden Angaben zu ihrer Arbeitsbelastung machen. Des Weiteren gibt es observatorische Methoden, bei denen die Arbeitsbelastungen von einem geschulten Beobachter evaluiert und oftmals über Punktesysteme (Scores) entsprechend notiert wird. Die Bepunktung ermöglicht eine Gesamteinschätzung von ergonomischen Risiken eines Arbeitsplatzes, ebenso können Risikoscores im zeitlichen Verlauf betrachtet werden.[117, 119] Der Beobachter kann die Evaluation anhand verschiedener Methoden durchführen, welche wiederum die ergonomischen Risiken unterschiedlich streng bewerten. Die Methoden können sich dabei z.B. auf die oben angeführten DIN- und ISO-Normen stützen. Eine zum Beispiel gängige internationale und wissenschaftlich fundiert Methode ist das „Rapid Upper Limp Assessment““ (RULA) [120], welches verhältnismäßig konservativ ist und Arbeitsabläufe- und -belastungen eher als ergonomisch riskant einstuft. Die Methode ermöglicht das Festlegen eines Körpergesamtscores, welcher Körperhaltung und Arbeitsbelastung des gesamten Körpers sowie insbesondere der NackenRumpf-, Schulterregionen, Arme und Hände berücksichtigt. Dabei fließen Zeit, Kraft und Repetition, mit den Haltungen eingenommen und Bewegungen ausgeführt werden, mit in die Wertung ein. Mehrere zahnmedizinische Studien [29, 121-123], in denen Z ̈̈ und ZFAs mit dem RULA evaluiert wurden, zeigten hohe Risikoscores, die als Handlungsempfehlung umgehende Anpassungen des Arbeitsfeldes suggerierten. In diesen Studien 
wurden Z̈̈ und ZFAs z.B. per Video aufgenommen [121] oder hochfrequent eingenommene Haltungen fotografiert $[122,123]$ und anschließend vom Untersucher nach RULA evaluiert. Ein anderes Beispiel ist der Quick Exposure Check (QEC) [124, 125]. Dieser evaluiert die Arbeitshaltung sowie Bewegungsfrequenz, Anstrengung, Exposition auf Vibrationen sowie psychosoziale Faktoren und ermöglicht Risikofaktoren am Arbeitsplatz für Rücken, Nacken oder obere Extremitäten zu erheben. Auch mit dem QEC wurden bei der Evaluation zahnmedizinischer Arbeitsplätze hohe Risikoscores festgestellt, die eine Anpassung erforderten.[121, 126] In Deutschland hat die Bundesanstalt für Arbeitsschutz und Arbeitsmedizin (BAuA) die sogenannten Leitmerkmalmethoden (LMM) entwickelt. Diese beinhalten Beurteilungsbögen zum Evaluieren von Halten, Heben, Tragen, Ziehen und Schieben von Lasten, zu manuellen Arbeitsprozessen, Ganzkörperkräften, Körperzwangshaltungen sowie Körperfortbewegung. Diese LMM werden auch von Sozial- und Unfallversicherungen anerkannt.[119, 127, 128] Eigenangaben und observatorische Methoden sind abhängig von den subjektiven Einschätzungen eines Probanden oder Untersuchers, dem es u.U. nur möglich ist, einen Gesamteindruck oder ein besonders einprägsames Ereignis festzuhalten und der somit die Risikobeurteilung verfälschen kann.[129]

Direkte Messungen ermöglichen es Arbeitsabläufe anhand objektiver Daten in ihrer Vollständigkeit zu erfassen. Außerdem ermöglichen sie die Risikobeurteilung observatorisch schwierig zu erfassender Körperbewegungen, wie z.B. der Handgelenke und Hände oder der Rumpfrotation.[129] Unterschiedliche Messsysteme eignen sich zur Evaluation der Ergonomie. Es lassen sich markerbasierte Systeme unterscheiden, bei denen multiple Kameras anhand definierter Marker die Bewegungen des Probanden erfassen. Diese Systeme sind sehr präzise, aber aufgrund des raumfordernden Aufbaus von Kameras gerade an räumlich begrenzten Arbeitsplätzen nicht umsetzbar. Nicht markerbasierte Systeme verwenden optische Sensoren, welche ohne Marker und ohne Kalibrierung funktionieren. Bei korrekter Sensorpositionierung können diese Systeme ebenfalls vergleichbar präzise Ergebnisse wie markerbasierte Systeme erzielen. Bei geringfügig falscher Platzierung liefern sie allerdings keine verlässlichen Ergebnisse mehr.[119, 130] Außerdem für direkte Messungen verwendbar sind Inertialsensoren.[115, 116, 119, 131] Diese Sensoren beinhalten u.a. Beschleunigungssensoren und Gyroskope und werden an fest definierten Stellen am Körper platziert. Aus der Gesamtheit der Sensoren kann am Computer ein biomechanisches Modell des Probanden erstellt werden. In einer Aufzeichnung der Tätigkeit 
am Arbeitsplatz können jetzt fortlaufend und kontinuierlich kinetische Parameter wie die Position von Körpersegmenten zueinander sowie daraus resultierende Gelenkwinkel mittels der Sensoren erfasst und anschließend ausgewertet werden.[119, 130] Mithilfe direkter Messungen wurde bereits eine Vielzahl von Arbeitsplätzen untersucht, wie Büros [115], Arbeitsplätze in der Autoindustrie [132] und Arbeitsplätze mit manuellen Tätigkeiten.[119, 133] Zum Beispiel untersuchten Ohlendorf et al. [2, 134] mit Hilfe von Inertialsensoren das ergonomische Risiko besonders häufig ausgeführter Tätigkeiten im Tagesgeschäft von Zahnärzten. Dabei stellten sie fest, dass ergonomisch ungünstige Haltungen vorwiegend während der Patientenbehandlung auftreten und dass ergonomisch designte Behandlungsstühle die Arbeitshaltung signifikant verbessern können.[2]

Um die Vorteile der einzelnen ERATs optimal zu nutzen und gleichzeitig ihre Nachteile auszugleichen macht es ggf. Sinn, verschiedene Werkzeuge zu kombinieren. Durch direkte Messungen erhobene objektive Daten können mithilfe der verfügbaren Modelle observatorischer Methoden ausgewertet werden. Dadurch lassen sich auch optisch schwierig zu erfassende Arbeitsabläufe und langandauernde Tätigkeiten objektivieren und bewerten.[135, 136] Ein großer Vorteil der Kombination beider Werkzeuge ist, dass Risikoscores kontinuierlich für alle erfassten Regionen erhoben werden und somit als Gesamtscore aber auch als Unterscore der einzelnen Regionen für die gesamte Messzeit sowie für einzelne Zeitabschnitte errechnet werden können. Dies erlaubt dem Untersucher auch den Anteil jeweiliger Risikoscores am Gesamtrisiko zu betrachten. Dem Untersucher wird somit eine präzisere und umfassendere ergonomische Risikobewertung möglich.[119] Vignais et al. [137] kombinierten die direkte Messung mittels Inertialsensoren mit der RULA Methode zur ergonomischen Risikobewertung von Aufgaben der Materialhandhabung. Dabei ermöglichte ihnen die detaillierte Auswertung der objektiven Daten lokale Risikoscores auszumachen, die Rückschlüsse auf besonders stark beanspruchte anatomische Strukturen zuließen. Die zeitliche Verteilung der Risikoscores erlaubte des Weiteren einzelne Tätigkeiten der Arbeitsabfolgen als ergonomisch besonders riskant einzustufen. Die Schwierigkeiten RULA an die erhobenen objektiven Daten anzupassen, z.B. aufgrund fehlender Schwellenwerte für Gelenkwinkel, wird von den Autoren dabei präzise beschrieben.[137] 


\subsection{Krafttraining und seine Relevanz in der Zahnmedizin}

Das Auftreten von muskuloskelettalen Beschwerden in der Zahnmedizin beginnt oftmals mit dem Ermüden der Haltemuskulatur von Rumpf und Schultern. Wenn diese, die Körperhaltung stabilisierenden Muskeln, ermüden, beginnt der Behandler in eine unergonomische, ungesunde Haltung zu verfallen, welche die Grundlage für Verletzungen bildet.[24] Aus diesem Grund sollten ZÄ und ZFAs spezifische Übungen zur Kräftigung der Haltemuskulatur durchführen, um eine gute körperliche Gesundheit zu gewährleisten. Dabei konnten verschiedene umfragebasierte Studien [8, 39, 138] den positiven Effekt einer besseren physischen Fitness und muskuloskelettaler Gesundheit auf die Prävalenz von Beschwerden zeigen. Geringere oder keine körperliche Betätigung wiederum waren mit einer erhöhten MSE-Prävalenz assoziiert. Unter kroatischen Studenten konnte gezeigt werden, dass die Teilnahme an einem Trainingskurs im Rahmen der universitären Ausbildung die Fitness der Teilnehmer deutlich verbessern konnte.[38] Außerdem hatten Probanden, die regelmäßig trainierten, signifikant weniger Schmerzen im unteren Rücken. In einer Befragung unter den Probanden während der Studie gaben 37\% an nur während des Trainingskurses regelmäßig Sport zu betreiben, 62,5\% der Teilnehmer bestätigten regelmäßige Rückenschmerzen.[38] Gopinadh et al. [139] beschreiben, dass in einer Umfrage unter $170 \mathrm{ZÄ}$ diese Physiotherapie $(39,1 \%)$ und Yoga $(28,6 \%)$ als wichtigste Faktoren zur Schmerzlinderung angaben.[139] Eine schwedische randomisierte Kontrollstudie zeigt, dass mit Training mittlerer bis hoher Intensität, die subjektiven Beschwerden der Oberkörperregion der Probanden sowie deren Blutglukosespiegel gesenkt werden konnten.[53] Trotz dem in vereinzelten Studien nachgewiesenem Erfolg, gibt es in der Zahnmedizin keine wissenschaftliche Einigkeit, welches körperliche Training adäquat ist, um MSE zu verhindern bzw. zu reduzieren. In der Literatur [5, 24, 41] werden z.B. Aerobicübungen, das Praktizieren von Yoga und Krafttraining empfohlen, allerdings ohne sich dabei auf experimentelle Daten zu stützen.

In der Sportwissenschaft ist bekannt: die Entwicklung einer gut ausgeprägten Muskulatur, dient aus präventiver Sicht der Entlastung und minimiert die Verletzungsgefahr des Halteund Stützapparates, insbesondere der Gelenke und Wirbelsäule[42]. Eine gut ausgeprägte Muskulatur als Stützkorsett ist Voraussetzung für jegliche Form körperlicher Aktivität.[43] Eine Vielzahl von Studien [140-143] an Leistungssportlern, welche ihren Körper höchsten physischen Belastungen aussetzen, hat gezeigt wie wichtig ein hohes Maximalkraftniveau für eine hohe körperliche Leistungsfähigkeit ist. Ein gezielter Aufbau von 
Muskelkraft ist für körperlich sehr Anspruchsvolle Tätigkeiten, neben seinem Charakter als Präventivmaßnahme, somit auch höchstrelevant zum Erreichen von einem hohen Leistungsniveau.[144-146] Auch im Rahmen der Rehabilitation kommt dem gezielten Muskelaufbau hier eine wichtige Bedeutung zu, um nach Verletzung, Krankheit oder degenerativer Veränderung den aktiven und passiven Bewegungsapparat wieder alltagstauglich zu machen. Durch das Training kommt es zu einer verbesserten Ansteuerung der Muskulatur über das Nervensystem, die entscheidende Basisgröße zur Steigerung der Maximalkraft ist dabei aber der Muskelquerschnitt.[42] In der Literatur kann ein hoher signifikanter Zusammenhang zwischen der isometrischen, dynamischen Maximalkraft und dem gemessenen Muskelquerschnitts festgestellt werden.[142, 147-149] Auf dieser Grundlage wird erkenntlich, dass ein Training zum Zweck der Prävention von Verletzungen sowie zur Leistungssteigerung bei Aktivitäten mit körperlich hohen Anforderungen auf Muskelwachstum ausgerichtet sein muss. Ein so genanntes Hypertrophietraining.[42] Die bereits beschriebenen hohen physischen Ansprüche aufgrund von unter Anderem prolongierten Einnehmen statischer Körperhaltungen mit isometrischer Anspannung der Muskulatur [3, 4, 12], die der Beruf an ZÄ und ZFAs stellt, macht diese Art des Trainings somit auch für diese Berufsgruppe höchstrelevant.

Dabei sind die Charakteristika eines Hypertrophietrainings in der Sportwissenschaft anhand der Belastungsnormativa, den Beschreibungsgrößen der Trainingsbelastung, definiert.[42, 43] Die Belastungsintensität beschreibt den Belastungsreiz, welcher während einer Übung auf den Bewegungsapparat einwirkt. In der gängigen Krafttrainingsliteratur [42, 150-152]liegen die Intensitätsvorgaben bei $60 \%$ bis $90 \%$ des 1RM. Das 1RM („one repetition maximum") beschreibt das Maximalgewicht, das bei nur einer Wiederholung bewegt werden kann.[42] Die Intensität entspricht der Höhe der Spannung, die an den Muskelfasern anliegt. Eine hohe Muskelspannung gilt als ausschlaggebender Einfluss auf Kraft-/Muskelzuwachs.[153, 154] Geringere Belastungsintensitäten unter 60\% des 1RM werden in der Literatur als zu gering beschrieben, um als adäquater Reiz für Kraft- bzw. Muskelmassezuwachs zu dienen.[155] Die Belastungsdauer beschreibt die Zeit, in der eine Trainingsübung auf den Bewegungsapparat einwirkt. Dabei ergibt sich die Dauer eines Satzes dementsprechend aus der Anzahl der Wiederholungen. Als optimal werden in der Literatur Wiederholungsanzahlen zwischen 6 und 15 für die Entwicklung von Muskelmasse empfohlen.[42] Die Belastungsdichte definiert sich als das Verhältnis und der 
zeitlichen Abfolge von Belastung- und Erholungsphasen. Als Einheit dient hier die Serienpause angegeben in Minuten oder Sekunden. Beim Hypertrophietraining soll eine interserielle Pause zum Hinauszögern der Ermüdung führen, um somit das Energiepotenzial der Muskulatur voll auszureizen.[156] Die Empfehlungen bezüglich eines konkreten Zeitraums gehen dabei auseinander. Mehrere Studien [42, 157, 158] stimmen darin überein, dass größerer Kraftzuwachs nach längeren interseriellen Pausen erzielt werden kann, da diese den ATP- und Keratinphosphatspeichern mehr Zeit zum wiederauffüllen geben. Häufig sind als optimal interserielle Pausen zwischen ein und fünf Minuten beschrieben, wobei aus der Literatur nicht genau hervorgeht, welcher Einfluss auf den Zugewinn an Muskelmasse besteht.[42] Der Belastungsumfang beschreibt die Gesamtheit der in einer Trainingseinheit gesetzten Belastungsreize und lässt sich anhand der Anzahl durchgeführter Sätze beschreiben. Die Literatur $[42,159,160]$ und trainingspraktische Erfahrung der vergangenen Jahrzehnte konnte dabei die eindeutige Überlegenheit eines Mehrsatzgegenüber eines Einsatztrainings zeigen. Des Weiteren scheinen hohe Belastungsumfänge erforderlich, um Veränderungen des passiven Bewegungsapparats zu erzielen.[161] Im Durchschnitt finden sich für ein Hypertrophietraining in der trainingswissenschaftlichen Literatur Empfehlungen im Bereich von 3 bis 6 Sätzen.[42] Die Belastungshäufigkeit beschreibt die Anzahl an Trainingseinheiten pro Trainingszyklus, z.B. angegeben in Trainingseinheiten pro Woche. Dabei divergiert die optimale Belastungshäufigkeit z.T. zwischen den Autoren [42, 162-164]. So wird unter Anderem diskutiert, ob Sportler mit einem höheren Trainingsniveau auch eine erhöhte Trainingsfrequenz benötigen oder ob diese aufgrund der höheren Belastungsumfänge längere Regenerationsphasen nötig haben.[162-164] Außerdem wird diskutiert, ob die involvierte Muskelmasse Einfluss auf die Trainingshäufigkeit haben muss. So sind einige Autoren der Ansicht, dass größere Muskelmassen längere Regenerationsvorgänge benötigen, auch im Hinblick auf die Gesamtbelastung des Organismus.[162, 164] Es kann angenommen werden, dass nach der aktuellen Literatur die optimale Belastungshäufigkeit für den Muskelaufbau bei zwei- bis drei Trainingseinheiten pro Woche liegt.[42]

Trotz der nur in sehr geringem Umfang vorhandenen Informationen zum Einfluss von körperlichem Training in der Zahnmedizin [38, 50, 53], konnten Studien [44-49, 52, 165 167] in Berufsgruppen ähnlicher Arbeitsverhältnisse den positiven Einfluss eines Krafttrainings bestätigen. In multiplen Interventionsstudien an Büroangestellten [46, 52, 165] 
mit Nacken- und Schulterbeschwerden konnte mithilfe eines Krafttrainings eine Reduktion der Schmerzen und der Schmerzintensität erreicht werden. Wie in der Zahnmedizin üben Büroangestellte ein Großteil ihrer beruflichen Tätigkeiten sitzend aus, im kurzem Arbeitsabstand zu ihrem Arbeitsfeld i.d.R. dem Computer. Dabei ist auch hier die Entwicklung von Arm-, Schulter- und Nackenbeschwerden üblich.[45, 165, 168]

Blangsted et al. [46] untersuchten 549 Büroangestellte, welche randomisiert in drei gleich große Gruppen in Krafttraings-, Allgemeintrainings- und Referenzgruppe aufgeteilt wurden. Die Probanden trainierten über 12 Monate drei Mal pro Woche je 20 Minuten; das Training fand dabei am Arbeitsplatz statt. Für das Krafttraining wurden verschiedene Übungen mit Kurzhanteln durchgeführt, die besonders auf die Schulter- und Nackenregion abzielten. Dabei konnte für die betroffenen Körperregionen sowohl für das Krafttraining als auch für das Allgemeintraining eine Reduktion der Intensität und Dauer der Schmerzsymptome $(\mathrm{p}<0,0001)$ festgestellt werden. Probanden, die vor Trainingsbeginn asymptomatisch waren, zeigten bei Folgeuntersuchungen die niedrigste Prävalenz für Nacken- und Schultersymptome, wenn sie in der Krafttrainingsgruppe waren. Ylinen et al. [52] untersuchten 180 Büroarbeiter mit chronischen, unspezifischen Nackenbeschwerden. Je 60 Probanden wurden randomisiert einem Krafttraining, einem Ausdauertraining in Form von Aerobic und einer Referenzgruppe zugeordnet. Die Krafttrainingsgruppe führt hierbei Nackenübungen mit einem Gummiband sowie Kurzhantelübungen zur Kräftigung der Schultern und oberen Extremitäten durch bei maximal 15 Wiederholungen und circa $80 \%$ des 1RM. Des Weiteren führten sie Übungen zur Kräftigung der Bein-, Rücken- und Rumpfmuskulatur gegen ihr eigenes Körpergewicht aus. Die Probanden sollten dabei drei Mal die Woche über einen Zeitraum von 12 Monaten trainieren. Sowohl das Krafttraining- als auch das Ausdauertraining zeigten nach 12 Monaten eine signifikante Reduktion der Schmerzintensität um mehr als 30mm auf einer Visuellen Analog-Skala [169] von 0 bis $100 \mathrm{~mm}(0 \mathrm{~mm}=$ keine Schmerzen, $100 \mathrm{~mm}=$ größter Schmerz $)(\mathrm{p}<$ 0,001). In der Krafttrainingsgruppe waren die gemessenen Maximalkraftwerte der Nackenmuskulatur 3 bis 4 Mal höher als die der Ausdauertrainingsgruppe. Nur die Krafttrainingsgruppe zeigte eine signifikante Verbesserung des Bewegungsumfangs in Lateralflexion, Flexion und Extension. Andersen et al. [165] untersuchte den Einfluss eines spezifischen Krafttrainings, welches direkt die betroffenen Muskelgruppen trainiert, auf Patienten mit chronischen Nackenschmerzen (diagnostiziert mit Trapeziusmyalgie). Nach 10-wöchigem Training konnte dabei zu einer signifikanten Reduktion der 
Schmerzintensität um 35mm auf einer Visuellen Analog-Skala von 0 bis $100 \mathrm{~mm}$ (p < 0,001) beschrieben werden. Spezifisches Krafttraining führte zu prolongierter Schmerzlinderung im Nacken.

Auch in Berufsgruppen, welche primär im Sitzen arbeiten und gekennzeichnet sind durch prolongierte statische Belastungen sowie monotone, repetitive Bewegungen der Hände und Arme, ist eine hohe Prävalenz von Beschwerden im Schulter-Nackenbereich sowie im unteren Rücken festzustellen.[166, 167, 170] Zebis et al. [49] untersuchten 537 Laborarbeiter, welche randomisiert in Trainings- und Kontrollgruppe aufgeteilt wurden. Das Krafttraining bestand aus fünf Kurzhantelübungen für Nacken-, Schulter- und Unterarmmuskulatur. Es wurden 8 bis 15 Wiederholungen bei 6 bis 12 Sätzen ausgeführt. Es wurde über 20 Wochen drei Mal die Woche für mindestens 20 Minuten trainiert. Das Training fand dabei am Arbeitsplatz statt und wurde von Zeit zu Zeit von Trainern überwacht. 85\% der Probanden führten das Trainingsprogramm jede Woche aus. Der Prä-Post-Vergleich zeigte in der Trainingsgruppe in der Nackenregion eine signifikante Reduktion der Schmerzprävalenz sowie eine Reduktion der Schmerzintensität von 49\%. Des Weiteren zeigte der Prä-Post-Vergleich für die Trainingsgruppe eine Reduktion der Schmerzprävalenz in der Schulterregion, wobei Arbeiter mit Schulterschmerzen in der Ausgangsmessung nach dem Training eine um den Faktor 3,9 höhere Wahrscheinlichkeit hatten keine Schmerzen mehr zu haben verglichen mit der Kontrollgruppe.

Der schmerzreduzierende Einfluss eines Krafttrainings ist des Weiteren auch in Berufen mittelschwerer körperlicher Arbeit beschrieben, bei denen, sitzende und-, stehende Haltungen sich abwechseln und z.T. große physische Arbeitsbelastungen auf die Arbeiter einwirken. Oldervoll et al.[47] untersuchten 65 Krankenhausmitarbeiter, welche in randomisiert einer Krafttrainingsgruppe, einer Aerobic-Trainingsgruppe und einer Kontrollgruppe zugeordnet wurden. Die Probanden der Trainingsgruppen trainierten für 15 Wochen zwei Mal die Woche je 60 Minuten. Das Krafttraining bestand dabei aus Übungen für Rumpf-, Arm/Schulter-, Hüft- und Beinmuskulatur, dabei wurden pro Übung 12 bis 15 Wiederholungen bei 2 bis 3 Sätzen durchgeführt. Zur Evaluation der Beschwerden wurde ein Schmerzindex verwendet, der sich aus den Regionen der Beschwerden, dem Andauern der Schmerzen sowie dessen Auswirkung auf Arbeit- und Freizeitaktivitäten errechnete. Der Schmerzindex reichte dabei von minimal 0 bis maximal 24 Punkte. Der Prä-Post-Test Vergleich konnte dabei sowohl für die Krafttrainingsgruppe als auch für 
die Aerobic-Trainingsgruppe eine signifikante Reduktion der Muskelschmerzen in Nacken, Schultern und Rücken zeigen, während die Kontrollgruppe nahezu unverändert blieb. In der Krafttrainingsgruppe wurde der Schmerzindex von 12.3 prä auf 5.3 post und somit um 57\% reduziert. In der Aerobic-Gruppe wurde der Schmerzindex von 13.5 prä auf 4.1 post und somit um 70\% reduziert. Auch in einer Folgeuntersuchung sieben Monate nach Training konnten signifikant weniger Schmerzen als vor der Intervention festgestellt werden. Der Schmerzindex lag für die Krafttrainingsgruppe mit 6.8 weiterhin 45\% unter dem Ausgangswert. In der Aerobic-Gruppe lag der Wert mit 5.7 weiterhin 58\% unter dem Ausgangswert. Die Studie zeigt, dass Aerobe Kapazität nicht wesentlich ist zur Schmerzreduktion bei MSE.[47]

Die Literatur [46-49, 52, 165, 166] zeigt, dass ein spezifisches Krafttraining die Prävalenz von Muskuloskelettalen Erkrankungen verringern und auch akut sowie chronisch auftretende Symptome und Schmerzen lindern kann.[171, 172] Außerdem ist erkennbar, dass für Berufe mit erhöhter Arbeitsbelastung ein spezifisches Krafttraining anderen Trainingsformen mindestens ebenbürtig und in einigen Aspekten überlegen ist.[46-49, 52, 165, 166] Dabei sind sich die beschriebenen Berufsgruppen im Anforderungsprofil an den Arbeiter der Zahnmedizin sehr ähnlich. Es ist folglich davon auszugehen, dass ein systematisches Krafttraining auch unter ZÄ und ZFAs zu einer Reduktion der Prävalenz und Intensität von muskuloskelettalen Beschwerden führen kann sowie, dass es ein adäquates Mittel zur Prävention von MSE ist. Notwendige Studien, die diese Annahme bestätigen fehlen allerdings in der zahnmedizinischen Literatur.

\subsection{Aktueller Literaturstand bezüglich der Auswirkung eines systematischen} Krafttrainings auf die ergonomische Arbeitsweise bei Zahnärzten und Zahnmedizinischen Fachangestellten

Obwohl weitgehend Einigkeit über die positiven Effekte physischer Aktivität auf die Reduktion von muskuloskelettalen Beschwerden herrscht, beruht dieser Konsens fast vollständig auf umfragebasierten Studien, die die Korrelation zwischen mangelnder physischer Aktivität und erhöhter Prävalenz von MSE beschreiben.[8, 39, 40] Interventionsstudien, die die Effektivität bestimmter Trainingsarten, spezifischer Übungen und systematischen Trainingsplänen beschreiben, sind nur bei der Zahnmedizin in Arbeitsbelastung und Arbeitshaltung verwandten Berufen beschrieben.[46, 47, 49, 165] Die Besonderheiten des zahnmedizinischen Arbeitsfeldes und ihre einzigartigen Anforderungen an 
ZÄ und ZFAs fordern hier dennoch die genaue Untersuchung des Effekts von körperlichem Training auf Z̈̈ und ZFAs mit muskuloskelettalen Beschwerden.

Die Ergonomie in der Zahnmedizin verfolgt das Hauptziel dem Behandler eine gesunde, entspannte, aufrechte und symmetrische Arbeitshaltung zu ermöglichen, um MSEs vorzubeugen bzw. bestehende Beschwerden zu entlasten und Schmerzen zu reduzieren. Der Großteil der aktuellen Literatur [4, 6, 12, 19, 28] über die ergonomischen Risikofaktoren der Zahnmedizin evaluieren diese ebenfalls nur auf Grundlage von erhobenen Umfragen unter ZÄ und ZFAs. Aktuelle klinische Studien [34-37, 51] zur Ergonomie in der Zahnmedizin beziehen sich zumeist auf verhältnispräventive Maßnahmen. Da bereits im Begriff Muskel-Skelett-Erkrankung, die Muskulatur an erster Stelle steht, ist es wenig verständlich, warum der Einfluss der Muskulatur in einer Berufsgruppe mit solch hoher Prävalenz so schlecht erforscht ist. Insbesondere, da diese Prävalenz trotz den verhältnispräventiven Interventionen und Innovationen weiterhin sehr hoch ist.

Während verhältnispräventive Maßnahmen in wenigen zahnmedizinischen Studien[37, 51] bereits zeigen konnten, dass sie anhand objektiver Daten zu einer ergonomischen Verbesserung führen, fehlen solche Daten für den Einfluss eines systematischen Trainings. Diese Lücken in der zahnmedizinischen Literatur machen es notwendig, den Einfluss eines systematischen Trainings auf die ergonomische Arbeitshaltung anhand von objektiven Daten zu untersuchen, da sowohl die körperliche Fitness als auch die ergonomische Arbeitshaltung entscheidenden Einfluss auf die Prävalenz und Intensität muskuloskelettaler Beschwerden haben.

\subsection{Arbeitshypothesen}

Wenige zahnmedizinische Studien [8, 39, 40] weisen bereits darauf hin, dass es einen Zusammenhang zwischen sportlicher Betätigung und einer reduzierten Prävalenz von MSE bei Berufen in der Zahnmedizin gibt. Der reduzierende Einfluss eines systematischen Krafttrainings auf MSE wurde des Weiteren schon für andere Berufsgruppen (z.B.: Büroangestellte, Laborarbeiter, Krankenhauspersonal) beschrieben, die in ihrer Arbeitshaltung und Arbeitsbelastung den zahnmedizinischen Berufen verwandt sind. [46-49, 165, 166] Werden die vielen Studien [3, 4, 6, 11, 18, 29, 67] zu ergonomischen Risikofaktoren und dem Berufsbild von ZÄ und ZFAs betrachtet, wird deutlich, dass die Natur der zahnmedizinischen Berufe und die mit ihr verbundene ergonomische Arbeitsweise 
ausschlaggebend zu sein scheint für die sehr hohe Prävalenz von MSE in diesen Berufsgruppen. Es ist folglich fraglich, ob Z̈̈ und ZFAs in ihrer tatsächlichen ergonomischen Arbeitsweise den vorgestellten ergonomischen Standards einer optimalen Ergonomie entsprechen können, für welche eine aufrechte sowie symmetrische Arbeitshaltung mit möglichst neutralen Gelenkwinkeln essentiell ist.[21-25, 97] In der Literatur wurde bereits gezeigt, dass ein systematisches Krafttraining subjektive Parameter wie z.B. die Prävalenz Muskuloskelettaler Beschwerden verbessern kann.[46-49, 165] Folglich bleibt zu untersuchen, ob auch ein direkter Einfluss auf die ergonomische Arbeitsweise besteht. Um den Einfluss eines systematischen Krafttrainings anhand objektiver Parameter im Prä-Post-Vergleich zu untersuchen wurden somit folgende Arbeitshypothesen formuliert:

1. Ein systematisches Krafttraining führt objektiv zu mehr Arbeitszeit in neutralen Gelenkwinkeln und Gelenkpositionen, gemessen mithilfe von direkten Messungen, in der
a. Körperregion des Nackens
b. Körperregion des Oberkörpers
c. Körperregion der Schultern und Oberarme

2. Ein systematisches Krafttraining führt zu einer verbesserten objektiven Bewertung der ergonomischen Arbeitsweise, bewertet mithilfe von RULA, für die
a. Körperregion des Nackens
b. Körperregion des Oberkörpers
c. Körperregion der Schultern und Oberarme

3. Ein systematisches Krafttraining führt objektiv zu weniger Arbeitszeit in gesundheitlich riskanten, ergonomischen Arbeitsweisen, bewertet mithilfe von RULA, für die
a. Körperregion des Nackens
b. Körperregion des Oberkörpers
c. Körperregion der Schultern und Oberarme 


\section{$3 \quad$ Material und Methode}

\subsection{Material}

\subsubsection{Probanden}

Das Probandenkollektiv bestand aus erwachsenen Frauen und Männern, die sich freiwillig zur Teilnahme an der Studie bereit erklärt haben. An der Studie nahmen 22 Probanden teil, davon 3 Männer und 19 Frauen. Das Probandenkollektiv lässt sich anhand der Berufsgruppen weiter unterteilen: 59,09\% der Probanden waren Zahnarzt/ärztin, 31,82\% Zahnmedizinische Fachangestellte und 9,09\% Studierende der Zahnmedizin (Abb. 7).
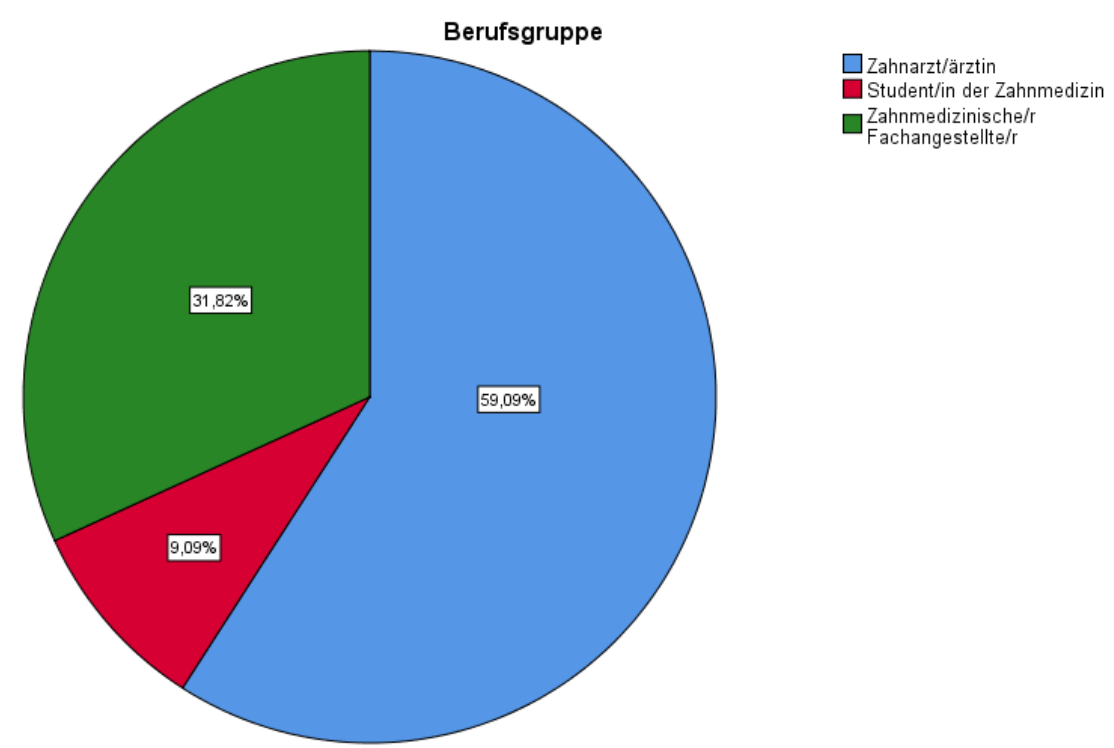

Abbildung 7: Verteilung Probandenkollektiv nach Berufsgruppe

Das Alter der Probanden lag zwischen 19 und 63 Jahren und im Mittel bei 38,52 \pm 14,12 Jahren (Mittelwert \pm Standartabweichung). Die Berufserfahrung in Jahren lag zwischen einem und 44 Jahren, mit einem Medianwert von fünf und einem Interquartilsabstand von 26,75 Jahren. Im Mittel waren die Probanden 169,24 \pm 7,15 cm groß. Das Gewicht der Probanden hatte einen Mittelwert von 64,67 $\pm 8,71 \mathrm{~kg}$.

Vor Studienbeginn wurden die Probanden außerdem regionenspezifisch zur Prävalenz muskuloskelettaler Beschwerden befragt, über die Zeiträume von zwölf Monaten, drei Monaten und sieben Tagen. Der prozentuelle Anteil der Probanden mit Beschwerden ist für die einzelnen Körperregionen und die jeweilige Prävalenz in der untenstehenden Tabelle 1 dargestellt. 
Tabelle 1: Prävalenz muskuloskelettaler Beschwerden

\begin{tabular}{|c|c|c|c|}
\hline & $\begin{array}{l}\text { 12-Monats Prä- } \\
\text { valenz [\%] }\end{array}$ & $\begin{array}{l}\text { 3-Monats Prä- } \\
\text { valenz }[\%]\end{array}$ & $\begin{array}{l}\text { 7-Tage Prä- } \\
\text { valenz }[\%]\end{array}$ \\
\hline Nackenregion & 86,4 & 72,7 & 63,6 \\
\hline Schulterregion & 81,8 & 68,2 & 40,9 \\
\hline Ellbogenregion & 4,5 & 9,1 & 4,5 \\
\hline Handgelenke/Hände & 40,9 & 36,4 & 31,8 \\
\hline $\begin{array}{l}\text { Oberer Rücken/Brust- } \\
\text { wirbelsäule }\end{array}$ & 31,8 & 31,8 & 22,7 \\
\hline $\begin{array}{ll}\text { Unterer } & \text { Rücken } \\
\text { (Kreuz/LWS) } & \end{array}$ & 63,6 & 59,1 & 36,4 \\
\hline Hüften/Oberschenkel & 22,7 & 13,6 & 13,6 \\
\hline Knie & 27,3 & 27,3 & 9,1 \\
\hline Knöchel/Füße & 0 & 0 & 0 \\
\hline
\end{tabular}

Die Probanden wurden des Weiteren gefragt, ob sie bereits zuvor an einer Rückenschule oder einer Ergonomieschulung teilgenommen haben. Außerdem wurde gefragt, ob die Probanden in ihrer Freizeit sporttreiben und ob sie dabei ein gezieltes Muskelaufbautraining betreiben. Der prozentuelle Anteil, der mit Ja oder Nein beantworteten Fragen ist unten in Tabelle 1 dargestellt.

Tabelle 2: Angaben zu Rücken-/Ergonomieschulung, Sport bzw. Training in Freizeit

$\begin{array}{lll}\text { Teilnahme an Rückenschule } & 22,7 & 77,3 \\ \text { Teilnahme an Ergonomieschu- } & 36,4 & 63,6 \\ \text { lung } & 100 & 0 \\ \text { Sport in Freizeit } & 36,4 & 63,6 \\ \text { Muskelaufbautraining } & \end{array}$

Für das systematische Krafttraining im Rahmen der Interventionsstudie wurden folgende Ein- und Ausschlusskriterien definiert:

Einschlusskriterien für die Teilnahme an der Interventionsstudie Krafttraining:

- Zahnarzt/ärztin

- Studierende der Zahnmedizin

- Zahnmedizinische/r Fachangestellte/r

- Auszubildender des Berufs Zahnmedizinische/r Fachangestellte/r

- Probanden (männlich/weiblich) im Alter von 18 bis 65 Jahren

- Beschwerden im Rücken- und/oder Nacken- und/oder Schulterbereich einmalig in den letzten zwölf Monaten (entsprechende Antwort im Fragebogen) 
Ausschlusskriterien für die Teilnahme an der Interventionsstudie Krafttraining:

- Aktuelle Verletzungen (z.B. Bandscheibenvorfälle, Wirbelsäulenverletzungen) im Kopfbereich oder im Bewegungsapparat

- rheumatische Erkrankungen

- Stark einschränkende Fehlformen (Skoliosen) der Wirbelsäule bzw. versteifte Wirbelsäulengelenke (krankhaft oder operativ bedingt)

- Genetisch bedingte Muskelerkrankungen

- Operationen, die weniger als 2 Jahre zurückliegen

- Einnahme von Muskelrelaxantien

Die Studie wurde von der Ethik Kommission der Goethe Universität Frankfurt am Main genehmigt (356/17).

\subsubsection{Messsystem}

Die kinematischen Messungen zur Erfassung der Körperhaltung während der Behandlungssimulation erfolgte mit dem inertialen Messsystem MVN BIOMECH Link der Firma XSens (Enschede, Niederlande). Es handelt sich um ein personengebundenes System, welches aus 17 Bewegungssensoren besteht, die über einen enganliegenden Lycra-Anzug am Probanden-Körper befestigt werden. Jeder Sensor ist dabei für ein spezifisches Körpersegment programmiert. Für die Messung werden die Bewegungssensoren zentral am Kopf über ein Stirnband, am Sternum und am Sacrum platziert. Des Weiteren bilateral an Schulter, Oberarm, Unterarm sowie Hand und am Ober-, Unterschenkel sowie Fuß platziert und über Kabel miteinander verbunden. Es handelt sich dabei um Inertialsensoren, die ausgestattet sind mit drei-Achsen Beschleunigungsaufnehmern, drei-Achsen Gyroskopen und drei-Achsen Magnetometer, welche Drehraten und Beschleunigungen messen können. Durch die Kombination mehrerer Sensoren können Lage- bzw. Winkelinformationen des gesamten menschlichen Körpers erhoben und ganze Bewegungen analysiert werden. Das System hat eine Abtastrate von $240 \mathrm{~Hz}$ und der Messfehler wird mit $\pm 1 \%$ vom Hersteller angegeben[54]. Die erhobenen Daten werden dann vom „Body Pack“, welches genau wie der Akku am Anzug befestigt ist, an einen Empfänger via WLan übermittelt. Dieser ermöglicht es die 
Daten am PC in Echtzeit und als 3D Animation mit der MVN ANALYZE SOFTWARE (Abb. 8) der Firma XSens (Enschede, Niederlande) darzustellen und auszuwerten.
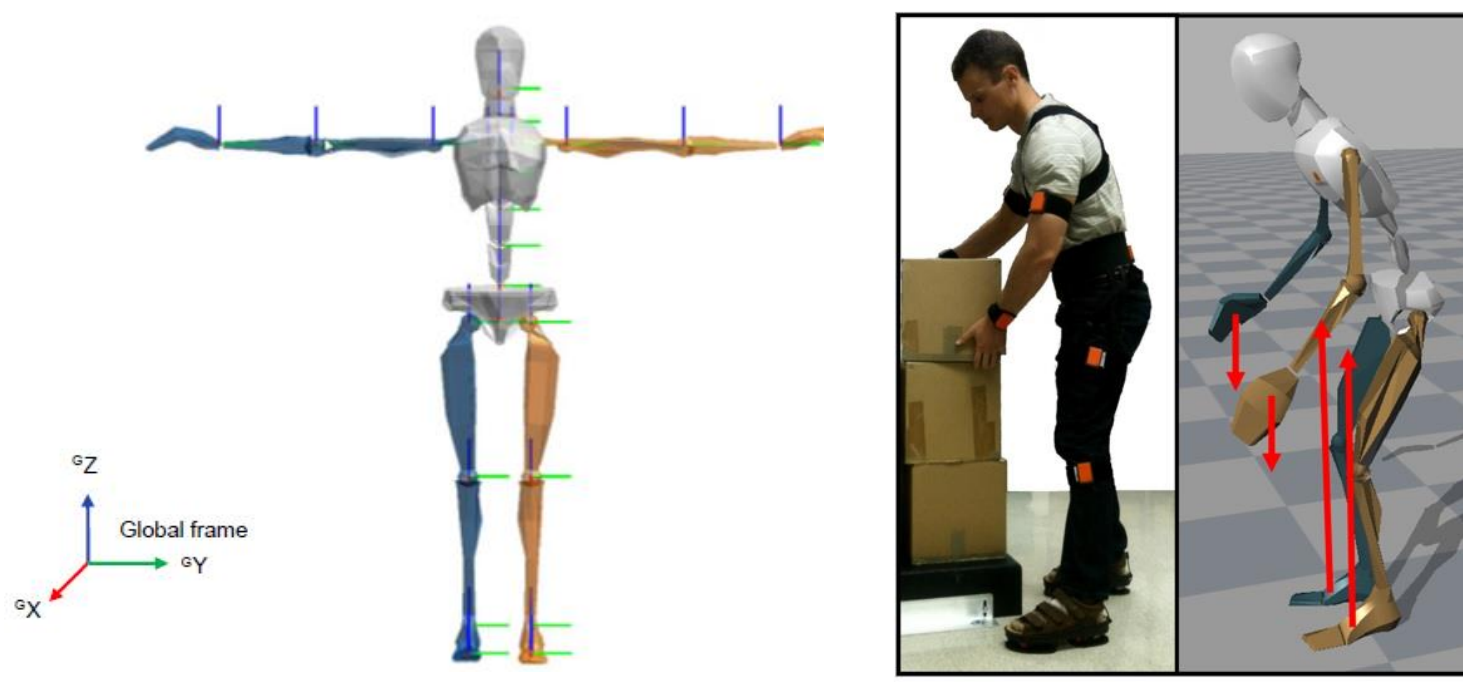

Abbildung 8: Animation in MVN ANALYZE [173]

\subsubsection{Datenauswertung}

Für die Auswertung der kinematischen Rohdaten wurde das Programm Matlab (MATrix LABoratory) der Firma The Mathworks (Natick, Massachusetts, USA) verwendet. Dies dient allgemein der numerischen Berechnung und Lösung mathematischer Probleme. Des Weiteren findet es Anwendung in der Datenerfassung bzw. Datenanalyse und ermöglicht auch die grafische Darstellung von Ergebnissen.

In Matlab erfolgte die Auswertung und Analyse, der mit Xsens erhobenen kinematischen Rohdaten, durch in Programmiersprache verfassten selbstgeschriebenen Routinen. Im Quellcode wurde dabei die für die ergonomische Beurteilung notwendigen Parameter definiert (Kapitel 3.2.4).

\subsubsection{SOPEZ Fragebogen}

Vor Beginn der Trainingsintervention füllte jeder Proband einen online Fragebogen aus. Der online Fragebogen wurde auf Grundlage des Nordic Questionnaire von Kuorinka et al. [174] und des Fragebogens nach Meyer et al. [175] erstellt und modifiziert. Dabei wurden aus dem Nordic Questionnaire Fragen über die Prävalenz Muskuloskelettaler Erkrankungen in den Regionen Nacken, oberer und unterer Rücken, Schultern, Ellenbogen, Handgelenke und Hände sowie Hüften/Oberschenkel, Knie, Knöchel und Füße erfasst. Dies geschah für eine Prävalenz über den vergangenen Zeitraum von sieben Tagen, drei 
Monaten, 12 Monaten und einer Lebenszeit [54]. Aus dem Fragebogen nach Meyer wurden Fragen bezüglich persönlichem/psychologischem arbeitsbedingtem Stress, Einschränkungen des Arbeitsumfeldes und Alltagsaktivitäten der Probanden ergänzt. Des Weiteren wurden Fragen zu muskuloskelettalen Vorerkrankungen und deren Behandlung sowie zu gesundheitsbezogenen Aktivitäten, wie z.B. vorangegangenem Ergonomietraining, Sport und Medikamenteneinnahme mit aufgenommen. Soziodemographische Daten wurden ebenfalls erhoben. Der SOPEZ Fragebogen wurde bereits im Rahmen der SOPEZ Studie zur Evaluation der Prävalenz Muskuloskelettalen Erkrankungen bei Zahnärzten und Zahnmedizinischen Fachangestellten verwendet. [10, 31, 54]

\subsubsection{Interventionstraining}

Für das Interventionstraining wurden, unter anderem, an Krafttrainingsgeräten der Firma Proxomed (Alzenau, Deutschland) trainiert. Darunter befanden sich Geräte aus der „Tergumed“-Linie, welche speziell für eine effiziente Therapie gegen Rückenschmerzen konzipiert sind. Genutzt wurden Maschinen zur Rückenflexion, Rückenextension, Lateralflexion und Rumpfrotation (Abb. 9 - 12). An diesen Maschinen wurde außerdem, eine isometrische Maximalkraftmessung durchgeführt, welche über einen angeschlossenen Computer in der entsprechenden Software ausgelesen werden konnte. Vom erzielten Maximalwert ausgehend wurde das Eingangsgewicht, mit dem der Proband trainierte festgelegt. Entsprechend einem Hypertrophietraining lag das Eingangsgewicht bei circa $80 \%$ der isometrischen Maximalkraftmessung[42]. 


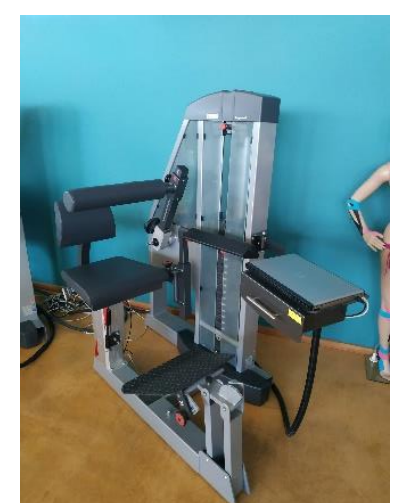

Abbildung 9: Rückenflexion

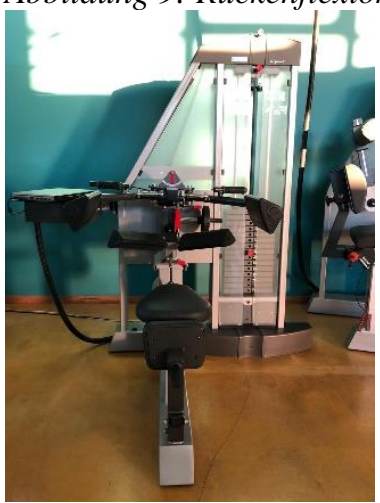

Abbildung 11: Lateralflexion

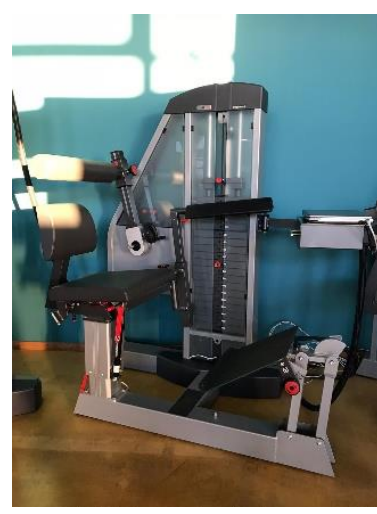

Abbildung 10: Rückenextension

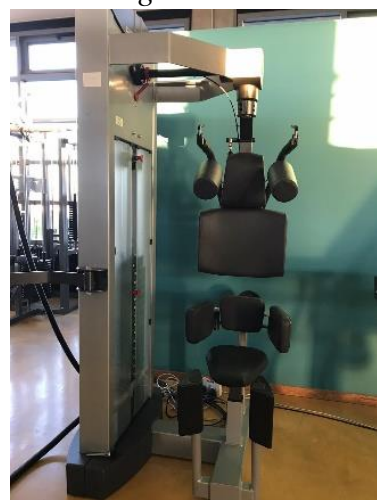

Abbildung 12: Rumpfrotation

Des Weiteren wurde an einer Beinpresse sowie einem Kabelzug trainiert (Abb. 13, 14). Diese Krafttrainingsgeräte gehörten zur „Compass“-Linie von Proxomed (Alzenau, Deutschland). Außerdem wurden für Übungen mit dem eigenen Körpergewicht eine Fitnessmatte, ein flacher Schaumstoffblock sowie ein Thera-Band® verwendet.

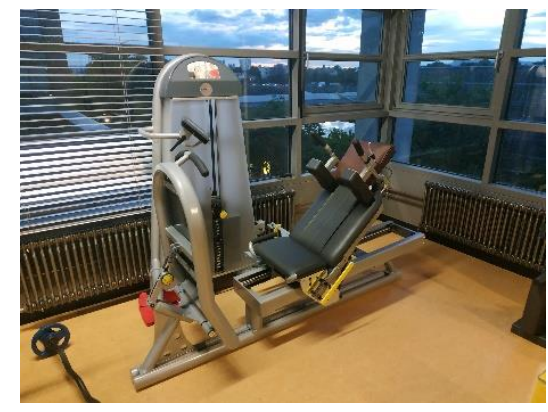

Abbildung 13: Beinpresse

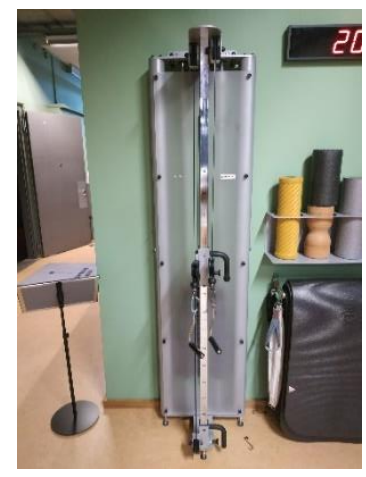

Abbildung 14: Kabelzug

Die Krafttrainingsintervention bestand aus einem 10- bis 12-wöchigen Trainingsprogramm, bei der wöchentlich zwei Trainingseinheiten à 60 Minuten durchgeführt wurden. Über den Trainingszeitraum mussten die Probanden minimal 18 und maximal 24 Trainingseinheiten absolvieren. Über den Trainingszeitraum durften die Probanden dabei maximal vier Einheiten verpassen, um nicht von der Studie ausgeschlossen zu werden. Verpasste Trainingseinheiten sollten dabei durch Ausweichtermine vor- oder nachgeholt 
werden. Zu Beginn der Trainingsintervention erfolgte eine Eingangsmessung zur Bestimmung der isometrischen Maximalkraft an den Krafttrainingsgeräten der „Tergumed“-Linie. Die einzelnen Übungen an den Geräten wurden in zwölf Wiederholungen à drei Sätze durchgeführt[42].

Das Training wurde in Kleingruppen von maximal vier Personen pro Trainer betreut. Die leitenden Trainer waren qualifizierte Sportwissenschaftler welche speziell für die Trainingsintervention geschult wurden. Über die Krafttrainingsintervention hinweg erfolgte eine sukzessive Steigerung der Belastung nach subjektiver Einschätzung der Trainer.

\subsection{Methode}

\subsubsection{Studienablauf}

Die biomechanischen Messungen, die im Rahmen der Studie durchgeführt wurden, fanden in den Einrichtungen des Instituts für Arbeitsmedizin, Sozialmedizin und Umweltmedizin des Universitätsklinikums Frankfurt am Main (Deutschland) statt. Dafür wurden in den Räumlichkeiten zwei zahnmedizinische Behandlungseinheiten installiert, an denen allen vier gängigen Basiskonzepte nach Kimmel [176, 177] simuliert werden konnten (Abb. 15). Die Krafttrainingsintervention fand in den Einrichtungen des Rehazentrums mediLoft (Frankfurt a.M., Deutschland) statt. 


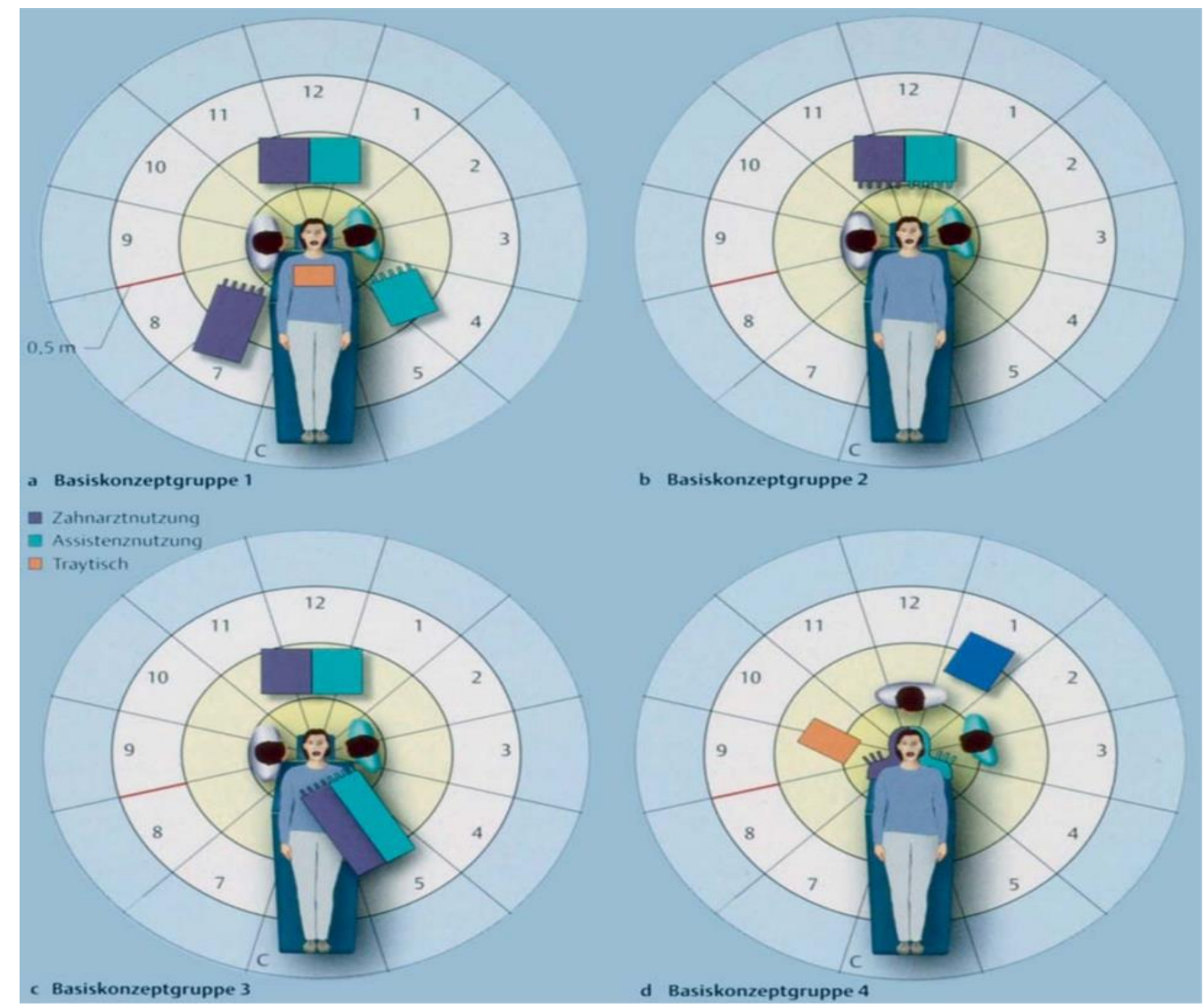

Abbildung 15: Basiskonzepte 1-4 nach Kimmel[177]

Zu Studienbeginn stand die schriftliche Einwilligungserklärung jedes Probanden, mit der dieser bestätigte, dass er über die Durchführung der Untersuchungen sowie über Natur, Bedeutung, Risiken und Tragweite der Studie aufgeklärt wurde. Des Weiteren unterschrieb der Proband, im Rahmen des Trainingsprogramms, dass ihm alle Trainingsgeräte erklärt und die Benutzung dieser erläutert wurden. Jeder Proband trainierte auf seine eigene Gefahr hin. Es wurde vereinbart, dass der Proband die Studie jederzeit auch ohne Angabe von Gründen verlassen könne.

Zuallererst füllten die Probanden online den SOPEZ Fragebogen aus, der Prävalenz muskuloskelettaler Beschwerden und weiterführende Belastungen und Einschränkungen des Arbeitsumfelds erfragte. Danach folgte die biomechanische Eingangsmessung, bei der jedes Behandlerteam am ihnen vertrauten Basiskonzept vermessen wurde und dabei eine ihrer Fachrichtung entsprechenden Behandlungssimulation durchführte (Kapitel 3.2.2). Zu Beginn des Trainingsprogramms stand am ersten Termin die Bestimmung der Maximalkraftwerte an den beschriebenen Geräten. Es folgte das 10- bis 12-wöchige Trainingsprogramm, mit jeweils zwei Einheiten pro Woche. Nach Abschluss des Trainingsprogramms 
erfolgte jetzt die biomechanische Ausgangsmessung für einen prä-post-Vergleich der arbeitsbedingten statischen und dynamischen Körperhaltungen. Dafür wurden die Probanden unter den exakt gleichen Bedingungen der Eingangsmessung vermessen. Die erhobenen Daten der biomechanischen Messungen wurden jetzt nach dem angepassten RULA Arbeitsbogen im Programm Matlab ausgewertet (Kapitel 3.2.4).

\subsubsection{Biomechanische Messung}

Um die biomechanischen Daten zu erheben, welche die für die Auswertung mit RULA (Kapitel 3.2.4) erforderlichen Informationen über Lage und Winkel der Gelenke liefern, erfolgte die Untersuchung während einer zahnmedizinischen Behandlungssimulation am Phantomkopf. Dafür wurden die Probanden in Behandlerteams von jeweils einem/r Zahnarzt/-ärztin und einem/r Zahnmedizinischen Fachangestellten (ZFA) gepaart und zusammen simultan vermessen (Abb. 16). Dabei erfolgten die Messungen in dem für das Behandlerpaar gewohnten Behandlungskonzept. Die aktuell bekannten vier Behandlungskonzepte unterscheiden sich grundsätzlich in der Anordnung der Elemente des zahnärztlichen Arbeitsplatzes und den im Behandlungsraum angeordneten Geräte und Schränke. Durch diese Anordnung wird das Arbeitsumfeld für den Zahnarzt sowie die ZFA bestimmt, der Bewegungsfreiraum eingeschränkt und Arbeitsabläufe in gewissem Maße vorherbestimmt. Nach Kimmel [176, 177] wird bei der Anordnung in ebendiese vier Basiskonzepte unterschieden, welche alle auf die sitzende Behandlung am Patienten ausgelegt sind (Abb. 15).

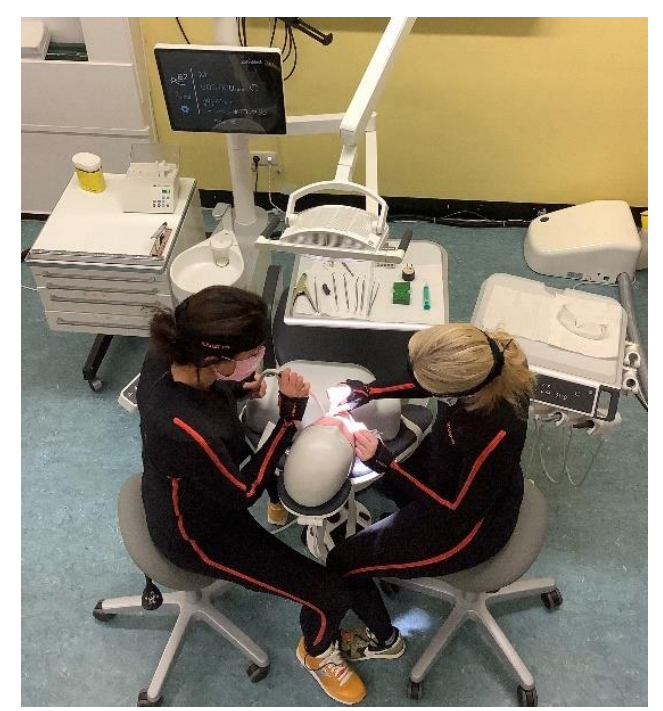

Abbildung 16: Behandlungssimulation Phantomkopf 
Das Behandlerteam wurde nun jeweils mit 17 inertialen Bewegungssensoren, des Systems MVN BIOMECH Link der Firma XSens (Enschede, Niederlande), ausgestattet, welche über einen Lycra Anzug am Probanden befestigt wurden. Der enganliegende Lycra-Anzug des Systems ermöglichte dabei zum einen die Sensoren reproduzierbar zu platzieren, zum anderen, dass die Sensoren während der Messung nicht in ihrer Position verändert wurden. Vorab der eigentlichen Messungen ist die Kalibrierung des Messsystems von Nöten. Die Kalibrierung diente der Zuordnung der Bewegungssensoren zum entsprechenden Körpersegment des jeweiligen Probanden. Das standardisierte Kalibrierungsprozedere erlaubt dem Programm, die individuelle Sensorposition und den Abstand zwischen einzelnen Sensoren zu erfassen. In der MVN ANALYZE SOFTWARE wurde dafür die Kalibrierung „Npose + Walk“ ausgewählt. Bei dieser Kalibrierung wurde der Proband zu Beginn in der „Npose“, als neutrale Ausgangshaltung, ausgerichtet. Für die „Npose“ nahm der Proband einen hüftbreiten Stand mit parallel ausgerichteten Füßen ein, der Rücken die Knie und die Arme waren durchgestreckt. Die Arme lagen dem Körper leicht an. Dann musste der Proband, auf Anweisung des Untersuchers, einige Schritte vorwärtslaufen, sich umdrehen, zum Ausgangspunkt zurückkehren und erneut die „Npose“ einnehmen [173].

Zur Simulation der Arbeitssituation wurden nun die Probanden nach ihrer jeweiligen Fachrichtung in Generalisten (allgemeintätige Zahnärzte), Oralchirurgen, Endospezialisten (Spezialisten für Wurzelkanalbehandlungen) und Kieferorthopäden einer spezifisch entworfenen Arbeitssimulation zugeordnet. Dabei erfolgte die Simulation in allen Fällen am Phantomkopf mit der Fachrichtung entsprechenden Kiefermodellen aus Kunststoff, welche bestückt waren mit passenden Kunststoffzähnen und Gingiva. Diese Kiefer werden eingeschraubt in den Phantomkopf, welcher mit einer Kunststoffmaske bestückt ist, die die Mundöffnung des Patienten simuliert. Die Probanden bearbeiteten jetzt über die Dauer von circa einer 25 Minuten eine vorgegebene Tätigkeitenreihenfolge, wobei definierte Schritte immer kurz zuvor durch den Untersucher angesagt und gemessen wurden. Dabei gab es jeweils eine Tätigkeit pro Kieferquadrant, welche wiederum in einzelne Arbeitsschritte segmentiert war.

Die Generalisten begannen mit einer Füllung des Zahns 16. Der erste Arbeitsschritt bestand in der Präparation einer Kavität mit rotierenden Instrumenten unter Verwendung von Keilen. Als zweites wurde eine Toffelmire Matrize dem Zahn angelegt und als dritte 
und letzter Schritt erfolgte das Legen einer Ketac®-Füllung unter Verwendung eines Heidemannspatels. Im zweiten Quadranten folgte die Präparation des Zahns 26 zur Aufnahme einer Krone in zwei Arbeitsschritten. Im ersten Schritt erfolgte die Reduktion des Zahns von okklusal, im zweiten Schritt eine zirkumferente Hohlkehlpräparation, jeweils unter Verwendung rotierender Instrumente. Die dritte Tätigkeit im dritten Quadranten bestand aus einer Wurzelkanalbehandlung des Zahns 35. Im ersten Schritt erfolgte die Präparation der Zugangskavität unter Verwendung rotierender Instrumente. Zweitens erfolgte das Auffinden der Kanaleingänge unter Verwendung einer Endofeile. Im dritten und letzten Schritt erfolgte das Aufbereiten und Spülen der Kanäle mit Endofeilen ISO 20-40 und Spülkanülen. Die Aufgabe im vierten Quadranten bestand im Entfernen von supra- und subgingivalem Zahnstein/Konkrementen unter Verwendung von Scalern und Küretten.

Im Falle der Endospezialisten bestand die Aufgabenstellung pro Quadrant jeweils in einer Wurzelkanalbehandlung des ersten Molaren. Es erfolgte also die Behandlung der Zähne 16, 26, 36, 46 mit gleicher Gliederung der Arbeitsschritte. Als erstes wurde jeweils ein Kofferdam um den Zahn gelegt. Im zweiten Schritt erfolgte die Präparation der Zugangskavität, sowie das Auffinden und Erweitern der Kanaleingänge. Zuletzt erfolgte die Aufbereitung und das Spülen der Kanäle auf eine gewisse Arbeitslänge, mit abschließender Entfernung des Kofferdams.

Die Oralchirurgen hatten die Aufgabe der chirurgischen Extraktion jeweils eines Zahns pro Quadrant. Im Oberkiefer erfolgte die chirurgische Extraktion eines palatinal impaktierten Zahnes 13. Der erste Arbeitsschritt war die palatinale und marginale Inzision von Regio 16 bis 13 unter Verwendung eines Skalpells. Im zweiten Schritt Darstellung des impaktierten Zahnes unter Verwendung einer chirurgischen Kugelfräse, sowie falls notwendig Teilung des Zahnes mittels einer Lindemann-Fräse. Dann Entfernen des Zahnes unter Verwendung eines Beinschen Hebels und/oder einer Dentalzange. Abschließend Kürretage des Zahnsäckchens. Analog erfolgte im zweiten Quadranten die Entfernung des vestibulär impaktierten Zahns 23. Im Unterkiefer mussten die Weisheitszähne 38 und 48 entfernt werden. Wobei sich das Vorgehen im ersten Schritt unterschied. Beim Zahn 38 erfolgte zuerst eine krestale Inzision in Regio 38 mit mesialer Entlastung. Hingegen erfolgte beim Zahn 48 eine krestale Inzision von Regio 48 bis 44 ohne Entlastung. Im zweiten Schritt erfolgte jeweils die Darstellung, Entfernung des Zahnes und Kürretage des Zahnsäckchens nach bereits beschriebenem Schema. 
In der Gruppe der Kieferorthopäden wurde die gleiche Aufgabe in allen vier Quadranten gestellt. Es erfolgte im jeweiligen Quadranten eine Multiband Behandlung mit Applikation von Brackets und einen Drahtbogen, sowie dessen Entfernung. Im ersten Schritt erfolgte das vorbereitende Anätzen der Zahnoberflächen der Zähne 1, 3, 4 und 6 im jeweilligen Quadranten. Als zweites wurde auf die Zähne Brackets geklebt und ein Drahtbogen eingeführt. Am Zahn 3 wurde im nächsten Schritt der Draht mit einer elastischen Ligatur integriert. Am Zahn 1 und 4 erfolgte die Integration mit einer Metallligatur. Im letzten Schritt erfolgte das Entfernen des Bogens und der Brackets von den Zähnen.

Auf diese Weise wird neben der individuellen biomechanischen Analyse synchron auch die Interaktion der beiden Probanden aufgezeichnet. Damit die Tätigkeiten exakt zugeordnet werden kann, wurde der gesamte Messablauf mit der objektiven Tätigkeitsanalyse simultan protokolliert und gleichzeitig in einer Totalansicht aus zwei Blickwinkeln gefilmt. Alle kinematischen Daten wurden im Anschluss mit denen der Tätigkeitsanalyse synchronisiert. Eine tabellarische Darstellung der spezifischen Tätigkeiten für die jeweilige Fachrichtung findet sich im bereits publizierten Methodenpaper über die gesamte SOPEZ-Studie [54].

\subsection{3 Übungen zur Kräftigung der stabilisierten Haltemuskulatur}

Für das Training wurden Übungen zur Kräftigung der stabilisierenden Haltemuskulatur ausgewählt, insbesondere im Rücken, Nacken, Schultern und Rumpf. Dies entspricht den, in der Literatur, vorherrschenden Regionen muskuloskelettaler Beschwerden unter Zahnärzten und zahnmedizinischem Fachpersonal.[6, 9, 12, 18]

Jedes Training begann mit einer fünfminütigen Aufwärmeinheit, die aus Treppen laufen bestand. Für das Krafttraining der Rücken-, Rumpf- und Beinmuskulatur wurden Übungen an Maschinen sowie Körpereigengewichtsübungen ausgewählt. Entsprechend des bereits beschriebenen Maximalkrafttraining wurden alle Übungen für drei Sätze à zwölf Wiederholungen ausgeführt, wobei zwischen den Sätzen jeweils eine Minute Erholungspause lag. Für den Rücken führte der Proband die Übungen Hyperextension an der Maschine, Lateralflexion an der Maschine und breites Rudern am Kabelzug aus (Abb. 17 19). 

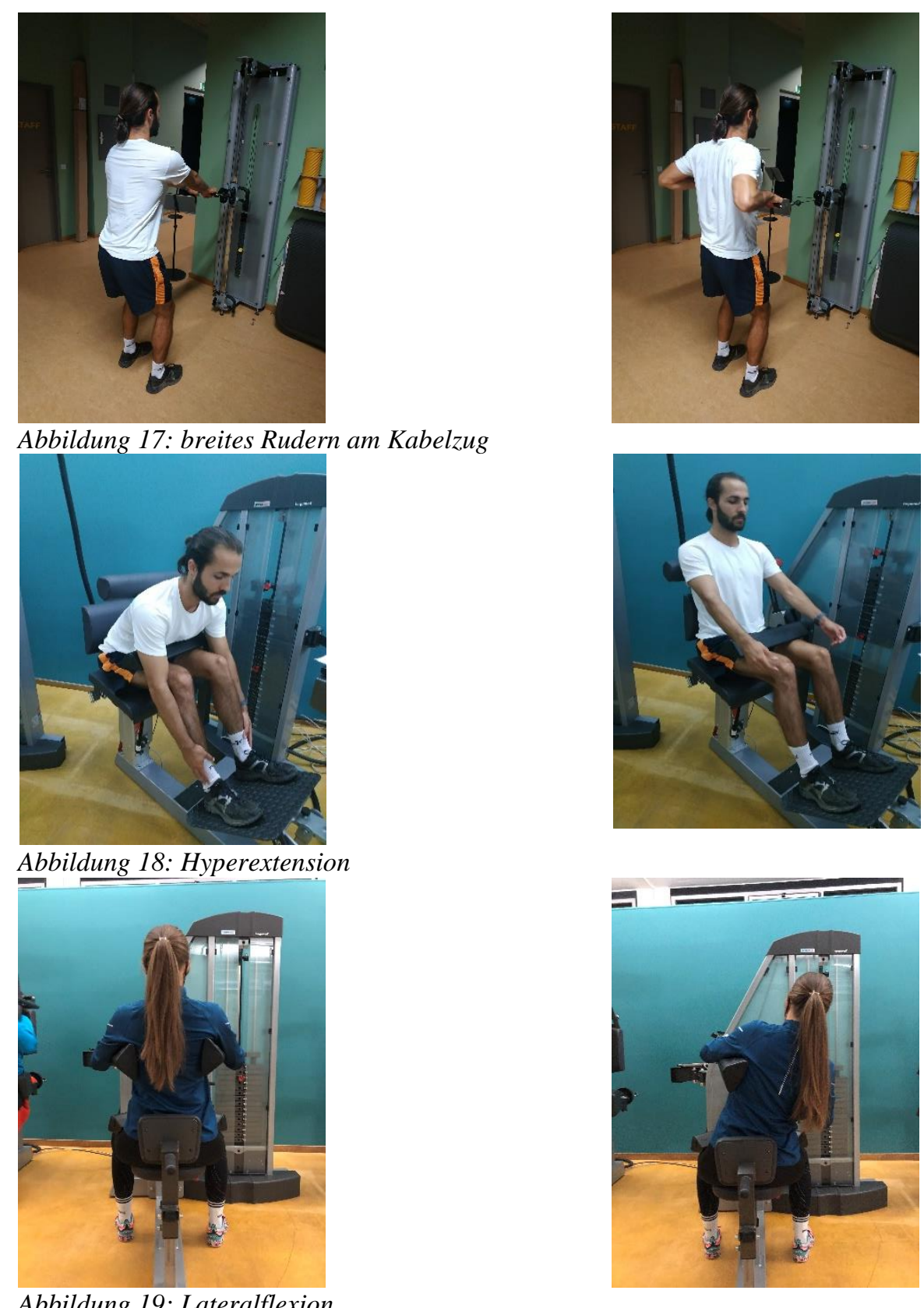

Für die Rumpfmuskulatur führte der Patient die Übungen Rumpfflexion an der Maschine und Rumpfrotation an der Maschine aus (Abb. 20, 21). Außerdem die Übung „Beine ablegen“, bei der der Proband auf dem Rücken liegt mit im 90-Winkel angezogenen Oberschenkeln und wiederum rechtwinklig abgewinkelten Unterschenkeln. Der Proband legt jetzt abwechselnd den linken oder rechten Fuß auf dem Boden ab und hebt ihn darauf wieder an in die Ausgangsposition (Abb. 22), zur Steigerung der Intensität kann die Übung über einen kurzen oder langen Hebel modifiziert werden, indem der Proband die Unterschenkel nicht abwinkelt sondern durchstreckt. 

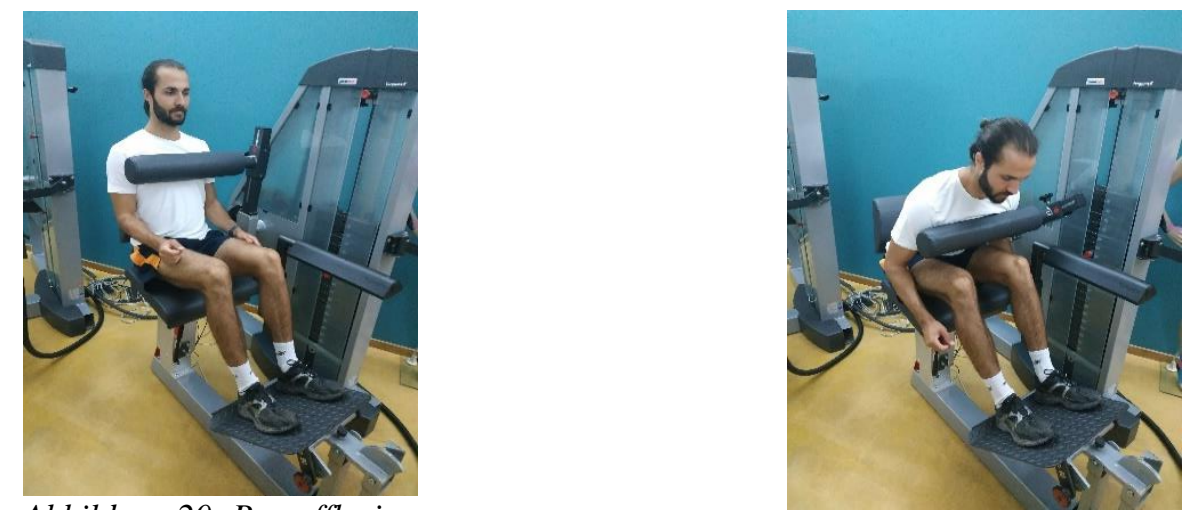

Abbildung 20: Rumpfflexion

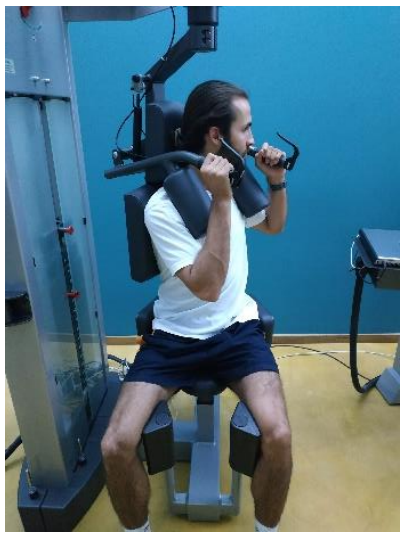

Abbildung 21: Rumpfrotation

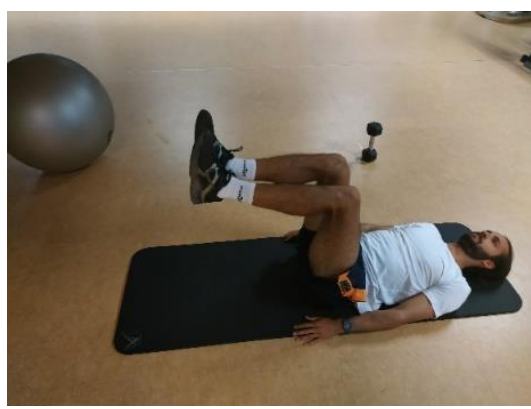

Abbildung 22: Beine ablegen

Für das Training der Beine trainierte der Patient an der Beinpresse und führte außerdem „Hipthrusts“, eine Brückenvariante mit aufliegenden Schulterblättern aus, zur Steigerung der Intensität konnte hier mit Gewichten oder einem Ball gearbeitet werden (Abb. 23, 24). Die Reihenfolge der Übungen wurde dabei randomisiert. 


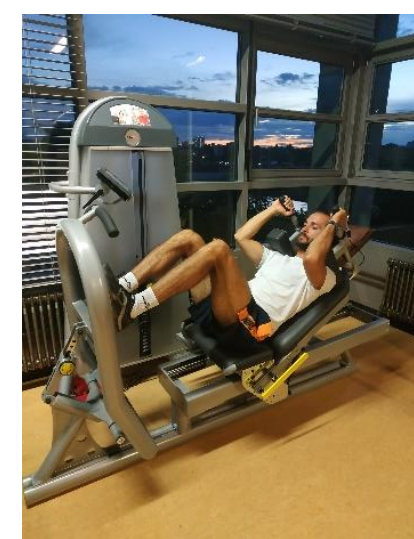

Abbildung 23: Beinpresse

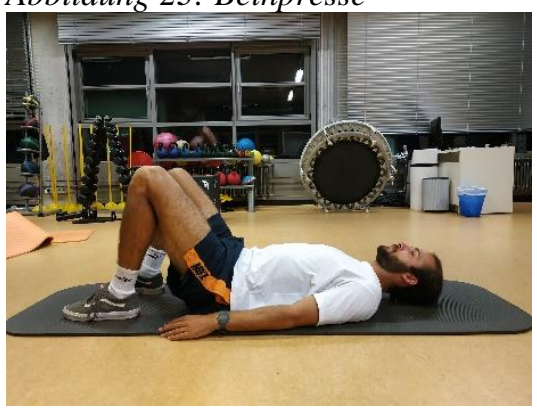

Abbildung 24: Hipthrusts
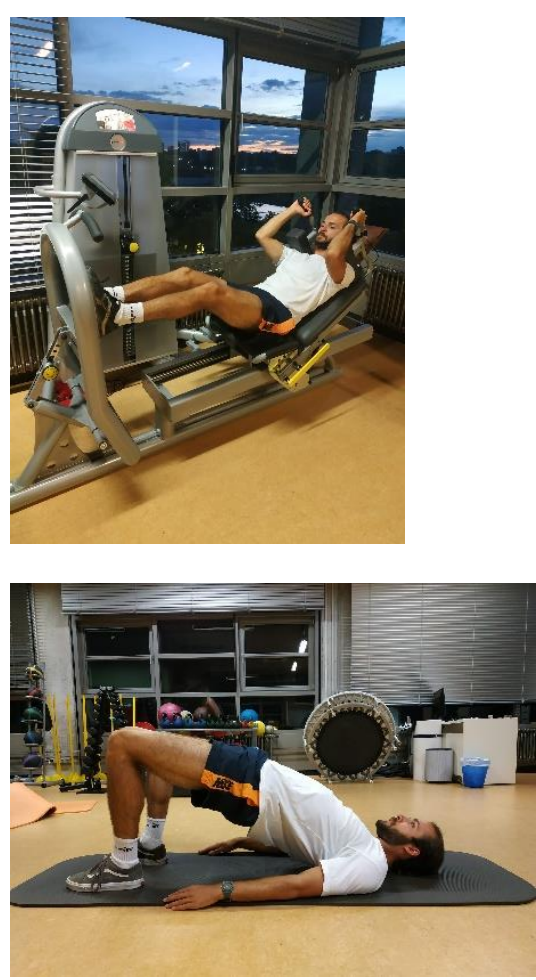

Zum Trainingsabschluss wurde ein Zirkeltraining bestehend aus drei Übungen über drei Sätze mit den Probanden durchgeführt, wobei die Belastung pro Übung jeweils abhängig vom Fitnesszustand des Probanden 20 bzw. 30 Sekunden dauerte und zwischen den Übungen 20 bzw. 30 Sekunden Pause lag. Zur Erhöhung der Intensität konnten die Zeiten je nach Fitnesszustand angepasst werden. Für die erste Übung, genannt „Wandwandern“, stand der Proband nah an einer Wand, mit den Handgelenken in einem geschlossenen Latex Band (Loop), die Handflächen zeigten dabei zum Probanden hin und mit rechtwinklig angezogenen Unterarmen wurden die Arme nun nach oben und unten bewegt (Abb. 24). Die Intensität der Übung konnte dabei durch die Stärke des Loops beeinflusst werden. Als zweite Übung, genannt „Superman“, lag der Proband bäuchlings auf dem Boden mit nach vorne ausgestreckten Armen (Abb. 26). Die Arme und Beine wurden jetzt gleichzeitig angehoben und abgesenkt, wobei eine Steigerung der Intensität durch ein diagonales Anheben und ein erhöhtes Zeitintervall erzielt werden konnte. Für letzte Übung des Zirkels, genannt „Arme wackeln“, stand der Proband hüftbreit mit gebeugten Knien und leicht vorgebeugtem Oberkörper. Die Arme sind ausgestreckt und der Proband wackelt abwechselnd mit den Armen auf und ab, wobei die Intensität durch die Zuhilfenahme von Gewichten und ein erhöhtes Zeitintervall erhöht werden konnte (Abb. 27). 


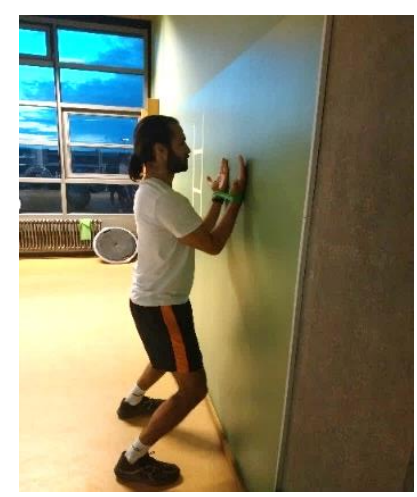

Abbildung 25: Wandwandern

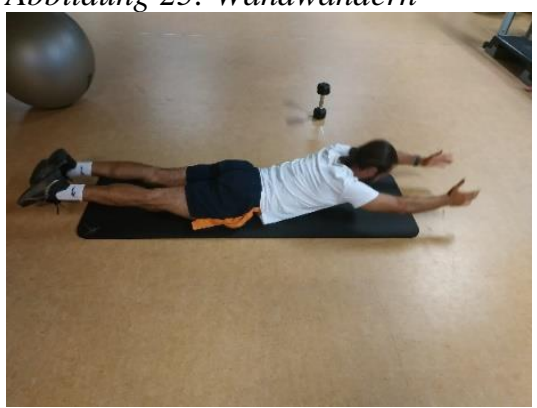

Abbildung 26: Superman

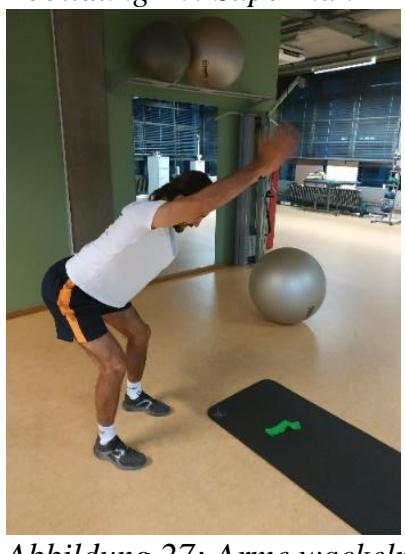

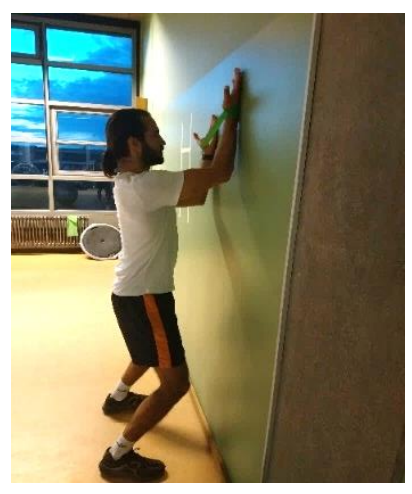
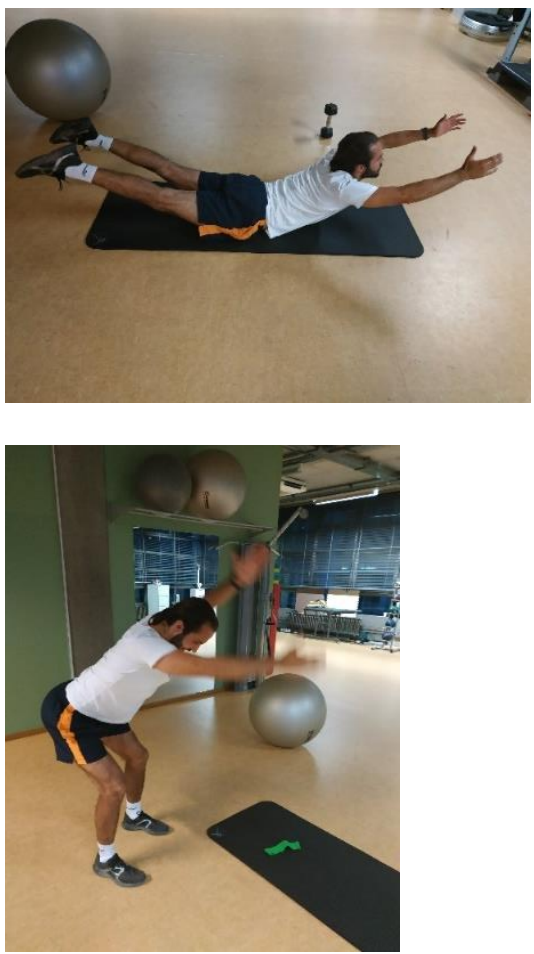

Abbildung 27: Arme wackeln

Die Anzahl der absolvierten Wiederholungen sowie die Intensität der Übungen jedes Probanden wurden für jede Trainingseinheit notiert, sodass sich die Trainingsbedingungen reproduzieren, anpassen und im Verlauf veranschaulichen lassen konnten.

\subsubsection{Auswertung der kinematischen Daten}

Als Grundlage für die Abschätzung des ergonomischen Risikos diente das „Rapid Upper Limp Assessement“ (RULA) [120], das von McAtamney und Corlett 1993 zur ergonomischen Begutachtung von Arbeitsplätzen entwickelt wurde. Das Verfahren wurde dazu entwickelt anhand eines Punktesystems (Scores) Handlungsempfehlungen abzuleiten, ob eine Veränderung am Arbeitsplatz aus ergonomischer Sicht notwendig ist bzw., ob weitere Untersuchung notwendig sind. 
Ursprünglich wurde RULA als observatorische Methode entwickelt, um die ergonomische Arbeitsweise an einem Arbeitsplatz zu bewerten und bezüglich ihrer gesundheitlichen Risiken zu evaluieren. Im Rahmen dieser Studie wurde mit objektiven Daten aus den biomechanischen Messungen gearbeitet, um computergestützt auf Basis des RULA Arbeitsbogens eine ganzheitliche und präzise Bewertung der Arbeitsweise von Z̈̈ und ZFAs zu errechnen.

Das RULA besteht konventionell aus einem Arbeitsbogen, welcher während der Untersuchung ausgefüllt wird. Dieser teilt die zu erhebenden Körperregionen in Region A Arm und Handgelenke, sowie B - Nacken Oberkörper und Bein. Der Region entsprechend lassen sich nun Teilwerte (Subscores) für Region A und B ermitteln. Die Belastung, ausgehend von der Körperhaltung und Bewegung, lässt sich für jeden Körperteil anhand der Piktogramme auf dem Arbeitsbogen (Abb. 28) ermitteln und dieser Belastung entsprechend wird ein Wert notiert. In der Region A wird zuallererst der Oberarm hinsichtlich seiner Extension nach hinten und Flexion nach vorne eingeschätzt. Anhand des Piktogramms und der dazugehörigen Winkel, lässt sich eine entsprechende Bepunktung vergeben, wird die Schulter nun angehoben oder abduziert wird ein Wert addiert; wird die Schulter hingegen abgestützt oder angelehnt wird ein Punkt subtrahiert vom zuerst ermittelten Wert. Daraus ergibt sich der Oberarmwert (Upper Arm Position), welcher in Tabelle A eingetragen wird.

Im nächsten Schritt wird nach gleichem Schema ein Unterarmwert (Lower Arm Position) ermittelt. Arbeitet der Unterarm außerdem über die Körpermitte hinaus bzw. ist nach innen oder außen rotiert, wird ein weiterer Punkt zum Unterarmwert addiert.

Im dritten Schritt wird die Extension und Flexion der Handgelenke beurteilt, wobei eine seitliche Abweichung der Handgelenke wiederum zur Addition von Punkten zum Handgelenkswert führt.

Zuletzt wird jetzt der Rotationswert (Wrist Twist) der Handgelenke beurteilt. Anhand der vier Haltungswerte der vermessenen lässt sich nun in der dazugehörigen Tabelle A der Haltungswert (Posture Score) für die Region A ablesen. Dieser wird nun mit den Werten für Muskelarbeit (Muscle Use Score) und Kraft/Last (Force/Load Score) verrechnet. Der Wert für Muskelarbeit beschreibt wie repetitiv oder statisch eine Haltung ist. Wird eine hauptsächlich statische Haltung für über zehn Minuten eingenommen oder eine Bewegung innerhalb einer Minute mehr als vier Mal wiederholt, wird ein Punkt addiert. Der 
Kraft/Last Wert beschreibt die Arbeitslast, die auf den Probanden während der Untersuchung einwirkt. Bei einer Last über $2 \mathrm{~kg}$ bis $10 \mathrm{~kg}$ mit intermittierter Belastung wird ein Punkt addiert. Ist die Belastung repetitiv oder statisch werden zwei Punkte addiert. Eine Belastung über 10kg oder eine schockartige Belastung führt zur Addition von drei Punkten. Somit wird miteinbezogen wie repetitiv oder statisch eine Haltung ist bzw. wie groß die einwirkenden Kräfte sind, bevor sich der Gesamtwert der Region A errechnet.

Analog ist das Vorgehen für die Region B. Auch hier lassen sich zuerst die Haltungswerte anhand der Piktogramme und Winkel ermitteln. Für den Nackenwert (Neckscore) wird wieder die Extension bzw. Flexion des Hals beurteilt, wobei eine Seitneigung oder Rotation jeweils einen weiteren Punkt addiert. Zweitens wird für den Oberkörperwert (Trunk Posture score) die Position des Oberkörpers bestimmt, eine Rotation oder ein Lehnen zur Seite addiert wiederum Punkte. Zuletzt wird der Beinwert (Leg score) erhoben, wobei darauf geachtet wird, ob Beine und Füße unterstützt sind oder nicht. Die drei ermittelten Teilwerte lassen sich nun wieder in der korrespondierenden Tabelle B eintragen und der Haltungswert für Region B kann ausgelesen werden. Wie in der Region A werden jetzt wieder die Werte für Muskelarbeit und Kraft/Last erhoben und addiert, um den Gesamtwert der Region B zu erhalten.

Mit den beiden Gesamtwerten A und B lässt sich jetzt in der Tabelle C die Gesamtpunktzahl auslesen. Anhand dieser kann eine direkte Handlungsempfehlung abgeleitet werden. Gesamtpunktwerte von 1 bis 2 bedeuten akzeptable ergonomische Verhältnisse, die keiner Änderung bedürfen. Werte von 3 bis 4 erfordern weitere Untersuchungen, da eine Veränderung notwendig sein kann. Werte von 5 bis 6 erfordern neben weiteren Untersuchungen eine baldige Veränderung. Bei einem Wert von 7 sind sofortige Veränderungen notwendig. 


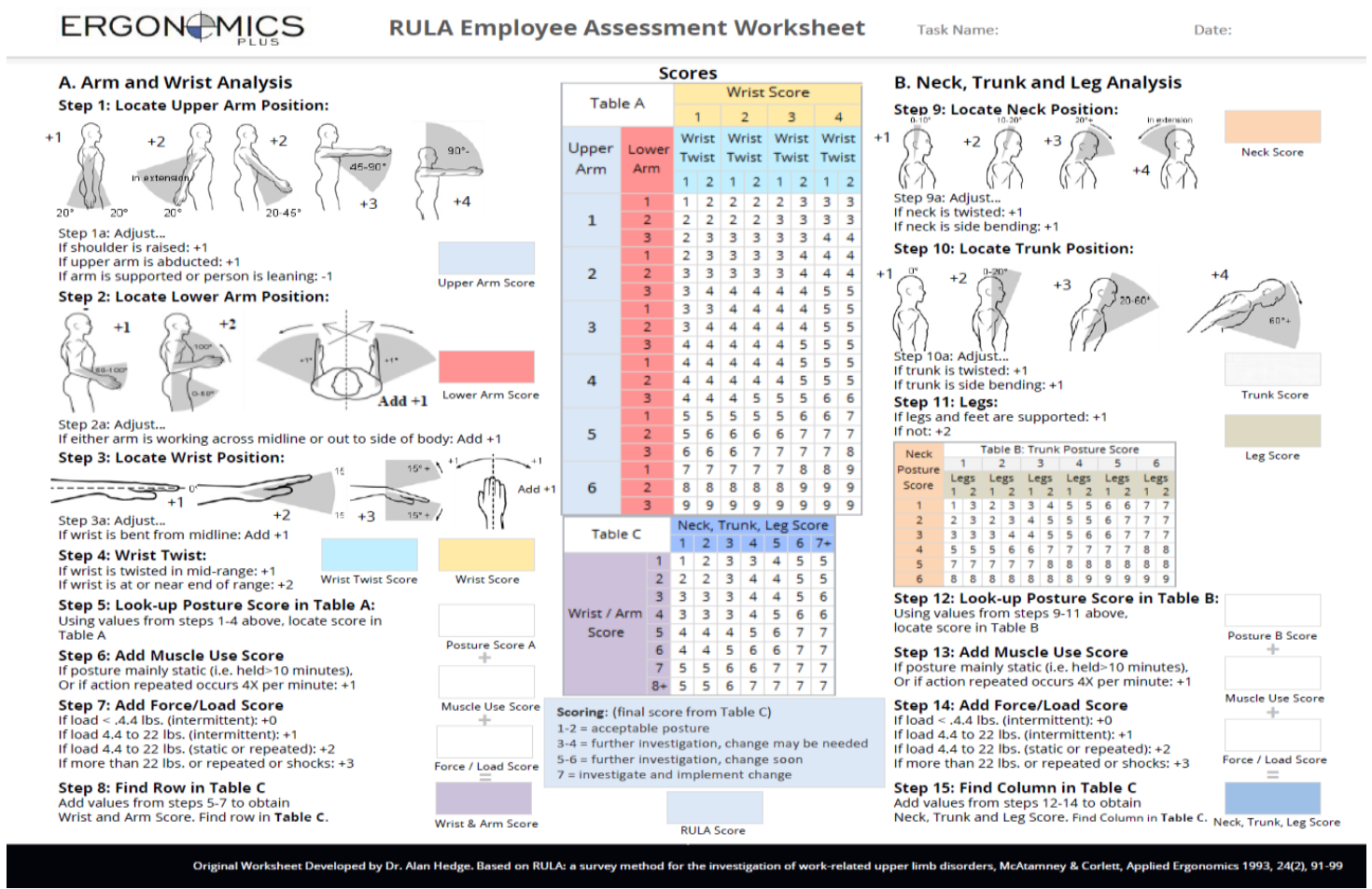

Abbildung 28: RULA Employee Assessment Worksheet

Für die automatische Auswertung über das Programm Matlab (MATrix LABoratory) der Firma The Mathworks (Natick, Massachusetts, USA) wurde der RULA Arbeitsbogen angepasst. Um die kinematischen Rohdaten mit der ursprünglich observatorische Methode RULA kompatibel zu machen, mussten fehlende Schwellenwerte z.B. für Gelenkwinkel und Bewegungsfrequenzen vor der Auswertung definiert und in im Quellcode des Auswertungsprogramms festgelegt werden. Dabei wurden Anpassungen am RULA Arbeitsbogen sowie auch pauschale Punktbewertungen auf Grundlage der praktischen zahnmedizinischen Tätigkeiten vorgenommen. Diese Anpassungen erfolgten dabei in Anlehnung an aktuelle Studienergebnisse [137] sowie den DGUV Informationen 208-053 „Mensch und Arbeitsplatz - Physische Belastungen“.[178] Die vorgenommenen Anpassungen werden im folgenden Abschnitt aufgeführt.

Für die Bewertung des Oberarms wurde ein Anheben der Schulter als eine Elevation des Schultergürtels $>5^{\circ}$ festgelegt. Eine Abduktion des Oberarms in der Frontalebene wurde definiert als Abduktion $>45^{\circ}$. Das Ablegen oder Anlehnen des Arms wurde aus der Bewertung entfernt. Bei der Unterarmposition wurden negative (-) Gelenkpositionen als arbeiten außerhalb der Körpermitte definiert. 0m entspräche bei diesem Parameter einer komplett neutralen Haltung des Unterarms. Bei der Haltung des Handgelenks, in der 
Sagittalebene, wurde für eine seitliche Krümmung die Radialdeviation (+) bzw. die Ulnadeviation (-) mit $>+10^{\circ}$ bzw. $<-10^{\circ}$ definiert.[137] Für die Bewertung des Wrist Twist (Rotation der Handgelenke) wurde die Bewertung +1 Punkt für Rotation im Neutralbereich $45^{\circ}$ bis $-45^{\circ}$ definiert. Sowie die Bewertung +2 Punkte für Rotation im endgradigen Bewegungsbereich $90^{\circ}$ bis $45^{\circ}$ und $-45^{\circ}$ bis $-90^{\circ}$ definiert.

Für Bewertung des Nackens wurde, in der Sagittalebene, eine Halsdrehung (Rotation) mit $>+10^{\circ}$ oder $<-10^{\circ}$ und eine Halsflexion, in der Frontalebene, ebenfalls mit $>+10^{\circ}$ oder $<-10^{\circ}$ festgelegt. Auch bei der Oberkörperhaltung wurde eine Rotation sowie eine Oberkörperneigung (Frontalebene) mit $>+10^{\circ}$ oder $<-10^{\circ}$ definiert.[137] Für die Bewertung der Beine wurde eine Bepunktung von +1 für unterstützte, ausgewogen belastete Füße/Beine und eine Bepunktung +2 für einbeinig, einseitig belastete Füße/Beine festgelegt.

Bei der Bewertung der Muskelarbeit wurde für statische oder repetitive Arbeit +1 Punkt addiert. Die statische Muskelarbeit wurde dabei auf Basis der Winkelgeschwindigkeiten ( $\omega)$ des Nackens (Halswirbelsäule) und des Unteren Rückens (Lendenwirbelsäule) in Zeitintervallen definiert. Der Anfang der statischen in Abgrenzung zur dynamischen Muskelarbeit wurde als $\omega<5 \%$ und das Ende als $\omega \geq 10 \%$ s oder Winkeldifferenz (Anfang, Ende) $\geq 7,5^{\circ}$ und Dauer $>10$ s definiert. Voraussetzung war dabei, dass alle drei Freiheitsgrade der Hals- und Lendenwirbelsäule $<5 \%$ s sein müssen um als statische Muskelarbeit gewertet zu werden. Für die repetitive Muskelarbeit wurde die Mean Power Frequency (MPF) betrachtet, wobei wiederum alle drei Freiheitsgrade berücksichtigt wurden. Das Kriterium für eine repetitive Arbeit wurde dabei definiert, dass eine Bewegung im entsprechenden Gelenk eine MPF über 0,5 Hertz anzeigt.[178]

Für die Kraft/Last Bewertung wurde eine intermittierte Belastung mit $<2 \mathrm{~kg}$ mit +0 Punkten sowie eine intermittierte Belastung mit 2 bis $10 \mathrm{~kg}$ mit +1 Punkten bewertet. Eine statische oder repetitive Belastung mit 2 bis $10 \mathrm{~kg}$ wurde mit +2 Punkten bewertet. Belastungen $>10 \mathrm{~kg}$ oder Erschütterungen bzw. Vibrationen wurden mit +3 Punkten bewertet. Für die Auswertung der Studienergebnisse im Prä-Post-Vergleich wurden bestimmte Schritte bzw. Scores ausgewählt. Verglichen wurden Upper Arm Position (Oberarmwert) rechts und links, der Lower Arm Position (Unterarmwert) rechts und links, der Wrist Position und Twist Score (Handgelenk- und Rotation swert) rechts und links, der Neckscore (Nackenwert), der Trunk Score (Oberkörperwert) und der RULA Gesamtwert. Für die jeweiligen Scores bzw. Teilscores wurden folgende Parameter erhoben: 
(1) Medianwerte, Quartile sowie Interquartilsabstände für Prä- und Postmessung

(2) Relative zeitliche Verteilung der Bewertungsparameter (RULA Scores) für Prä- und Postmessung

(3) Relative zeitliche Verteilung der riskanten Bewegungsparameter (RULA Scores) für Prä- und Postmessung

(4) Relative zeitliche Verteilung der Gelenkwinkel und Gelenkpositionen für Prä- und Postmessung

\subsubsection{Statistische Auswertung}

Nach der Auswertung der kinematischen Rohdaten durch Mathlab wurden die Daten jedes Probanden für alle Parameter als Tabelle im Programm Microsoft Excel (2019) ausgegeben. Die statistische Auswertung erfolgte mit den Programmen BiAS Version 11.12 (Epsilon Verlag, Darmstadt, Deutschland, 2020) und IBM SPSS Statistics Version 26 (IBM, New York, USA, 2019). Mit dem Programm BiAS wurden die erhobenen Daten anhand des KOLMOGOROFF-SMIRNOFF-LILLIEFORS-TEST (KSL-Test) und dem korrigierten p-Wert zum KSL-Test nach Dallal und Wilkinson auf Normalverteilung überprüft. Der korrigierte p-Wert zum KSL-Test nach Dallal und Wilkinson wurde zur genaueren Beurteilung bei $\mathrm{p}<0,1$ im KSL-Test angewandt. Überprüfte Parameter mit $\mathrm{p}$ $<0,05$ für den jeweiligen Test waren nicht normalverteilt. Keiner der überprüften Parameter unterlag einer Normalverteilung.

Im Programm Microsoft Excel wurden für die einzelnen Auswertungsparameter die Medianwerte, das erste Quartil und dritte Quartil sowie der Quartilsabstand errechnet.

Mit dem Programm IBM SPSS Statistics erfolgte der Prä-Post-Vergleich der jeweiligen Parameter sowie ein Seitenvergleich zwischen rechts und links der Oberarm-, Unterarm, Handgelenk \& Rotation s-Regionen. Für die jeweils verbundenen Stichproben der Präund Postmessung bzw. rechten und linken Körperhälfte wurden nicht parametrische Tests durchgeführt. Entsprechend der Nicht-Normalverteilung wurde der Wilcoxon-MatchedPairs Test angewandt. Der Wilcoxon-Matched-Pairs Test ist ein Vorzeichen-Rang-Test der sowohl die Richtung als auch die Höhe der Differenzen zwischen zwei Stichproben miteinschließt. Er überprüft die gepaarten Stichproben auf die Gleichheit ihrer Grundgesamtheit anhand der Nullhypothese. Die Nullhypothese geht davon aus, dass es zwischen den Medianwerten der Differenzen beider Stichproben keine signifikanten Unterschiede gibt. Das Signifikanzniveau ist $\mathrm{p} \leq$ bei 0,050 definiert. Liegt die Signifikanz bei $\mathrm{p} \leq 0,05$, 
wird die Nullhypothese abgelehnt und es liegt ein signifikanter Unterschied zwischen den Stichproben vor. Um bei multiplen Tests das Signifikanzniveau $\alpha$ anzupassen und dem Effekt der Alpha-Fehler-Kumulierung entgegen zu wirken, welche die Fehlerwahrscheinlichkeit erhöht und zu irrtümlich signifikanten Ergebnissen führen kann, wurde außerdem die Bonferroni-Holm-Korrektur angewandt.[179] Dabei wird das angestrebte Signifikanzniveau $\alpha$ durch die Anzahl der durchgeführten Tests geteilt, um auf das angepasste Signifikanzniveau $\alpha$ für den einzelnen Test zu kommen.

Für eine genaue und nicht vereinfachte statistische Überprüfung der kinematischen Rohdaten, wurden die jeweiligen Gelenkwinkel mithilfe des Systematic Parametric Mapping überprüft.[180] Alle statistischen Analysen erfordern ein Zufallsmodell auf dessen Grundlage die Wahrscheinlichkeit berechnet wird, dass zufällige Datensätze das beobachtete Ergebnis erzielen.[181] Diese Methode beruht auf der Erkenntnis, dass für die korrekte statistische Überprüfung von mehrdimensionalen Datensätzen, wie z.B. spatiotemporalen (raumzeitliche) Datensätzen, eine Anwendung einer ebenso mehrdimensionalen Zufallsmethode nötig ist. Mit Hilfe des Systematic Parametic Mapping (SPM) erfolgt eine kontinuierliche Überprüfung der Datensätze ohne, dass es zu Abstraktion von Informationen kommt, um den Datensatz mit der statistischen Methode kompatibel zu machen.[181, 182] Das SPM überprüfte die Gelenkwinkel der Prä- und Postmessung anhand eines gepaarten T-Tests. Der gepaarte T-Test untersucht die beiden Stichproben auf den Mittelwert eines ausgewählten Merkmals. Der gepaarte T-Test wurde im SPM mit einem Bootstrapping-Verfahren durchgeführt. 


\section{$4 \quad$ Ergebnisse}

In diesem Kapitel werden im Zuge der biomechanischen Messungen erhobene und nachfolgend ausgewertete Parameter dargestellt. Die Ergebnisse der durchgeführten Auswertung werden anhand der im RULA Arbeitsbogen vorgegebenen Reihenfolge präsentiert. Für die ausgewerteten Regionen bzw. Scores werden die Unterschiede zwischen Prä- und Postmessung der Probanden anhand des Medianwertes, den oberen und unteren Quartilen sowie dem Quartilsabstand beschrieben. Da sich diese Scores aus der relativen zeitlichen Verteilung der Bewertungsparameter errechnen, wird auch der Unterschied zwischen der relativen Zeitverteilung der Scores dargestellt. Ergänzend erfolgt zusätzlich für einzelne Regionen die Darstellung der relativen Zeitverteilung als riskant eingestufter Scores (Risky Scores) zwischen Prä- und Postmessung. Abschließend werden die Ergebnisse der gemessenen Gelenkwinkel und Gelenkpositionen nach ihrer relativen zeitlichen Verteilung präsentiert.

\subsection{Prä-Post-Auswertung der Medianwerte und Quartilsabstände}

4.1.1 Prä-Post-Auswertung der Medianwerte und Quartilsabstände - Oberarm, Unterarm, Handgelenk \& Rotation linke Körperhälfte

Tabelle 3 zeigt die gebildeten Medianwerte und Quartilsabstände aller Probanden der RULA Scores für die linke Körperhälfte. Statistisch kann mit dem Wilcoxon-MatchedPairs Test kein signifikanter Unterschied $(\mathrm{p} \geq 0,05)$ zwischen den untersuchten Werten der jeweiligen Messungen beschrieben werden. Für den linken Oberarm liegt die Signifikanz bei $\mathrm{p}=0,70$ Im Vergleich von Prä- und Postwerten des linken Oberarms (Abb. 29) hat sich der Median marginal von 1 auf 1,5 erhöht. Die ergonomische Risikoeinschätzung anhand des RULA Scores für den linken Oberarm änderte sich dadurch nicht und blieb unter 5, der definierten Risikoschwelle. Das erste Quartil (unteres Quartil) lag vor und nach dem Interventionszeitraum bei 1 und hat sich nicht verändert. Im dritten Quartil (oberes Quartil) hat sich der Wert von prä 1,5 auf post 2 geringfügig erhöht. Der Quartilsabstand zwischen dem 1. und 3. Quartil hat sich für den linken Oberarm ebenfalls von 0,5 auf 1 erhöht. 
Tabelle 3: RULA Scores - Median- und Quartilswerte Linksseitig (Left) Oberarm (Upper Arm), Unterarm (Lower Arm), Handgelenk \& Rotation (Wrist Position and Twist)

Fett Markierung: Score wird gesundheitlich riskant eingestuft

\begin{tabular}{llllll}
\multicolumn{2}{l}{ RULA Left Upper } & \multicolumn{2}{l}{ RULA Left Lower } & \multicolumn{2}{l}{ RULA Left } \\
\multicolumn{2}{c}{$\begin{array}{l}\text { Arm Position } \\
\text { prä }\end{array}$} & post & \multicolumn{2}{l}{ Arm Position } & \multicolumn{2}{l}{$\begin{array}{l}\text { Postion and Twist } \\
\text { prä }\end{array}$} & post & prä & post \\
1 & 1 & 2 & 2 & 4,5 & 4 \\
1 & 1,5 & 2 & 2,5 & $\mathbf{5}$ & $\mathbf{5}$ \\
1,5 & 2 & $\mathbf{3}$ & $\mathbf{3}$ & $\mathbf{5}$ & $\mathbf{5}$ \\
0,5 & 1 & 1 & 1 & 0,5 & 1
\end{tabular}

Die statistische Signifikanz des linken Unterarms liegt bei $p=1,000$. Die Medianwerte für den linken Unterarm (Abb. 30) haben sich von einem Präscore bei 2 geringfügig auf 2,5 im Postscore erhöht. Die ergonomische Risikobeurteilung für den linken Unterarm änderte sich dadurch nicht und blieb unter 3 der definierten Risikoschwelle. Das untere Quartil war unverändert bei einem Score von 2. Auch das obere Quartil war im Präscore bei 3 und blieb auch im Postscore unverändert bei einem Wert von 3 somit wurde die ergonomische Arbeitsweise von jeweils 25\% der Probanden als gesundheitlich riskant eingestuft. Der Quartilsabstand änderte sich nicht und war weiterhin bei 1.

Für den linken Handgelenks- \& Rotationswert liegt die statistische Signifikanz bei p=0,465. Der RULA Medianscore (Abb. 31) hat sich im Vergleich vor und nach dem Interventionszeitraum nicht verändert und bleibt bei einem Wert von 5. Sowohl der Präals auch der Postwert erreichen somit die Riskoschwelle, die bei 5 definiert ist. Anhand der Medianwerte wird die Arbeitsweise für Handgelenk \& Rotation unverändert als gesundheitlich riskant eingestuft und erfordert nach dem RULA Arbeitsbogen eine umgehende Korrektur. Das erste Quartil verringert sich geringfügig von einem Präscore von 4,5 auf 4 im Postscore. Im dritten Quartil lässt sich keine Veränderung beschreiben. Der Quartilsabstand erhöht sich geringfügig von 0,5 auf 1. 


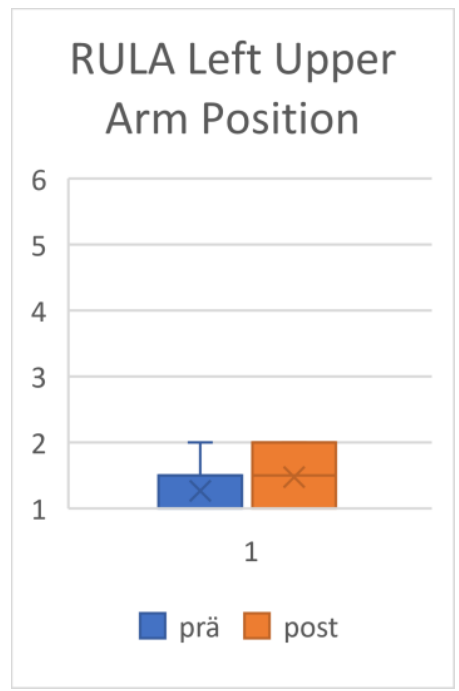

Abbildung 29: RULA linker Oberarmwert

Beschriftung der Ordinate beginnt bei niedrigmöglichstem Punktwert (1) und endet beim Höchstwert (6) dieser Region.

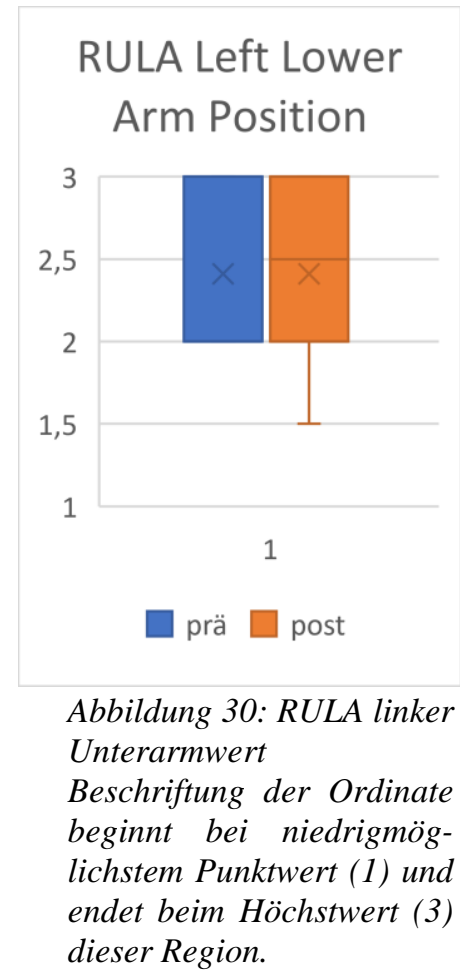

RULA Left Wrist

Postion and Twist

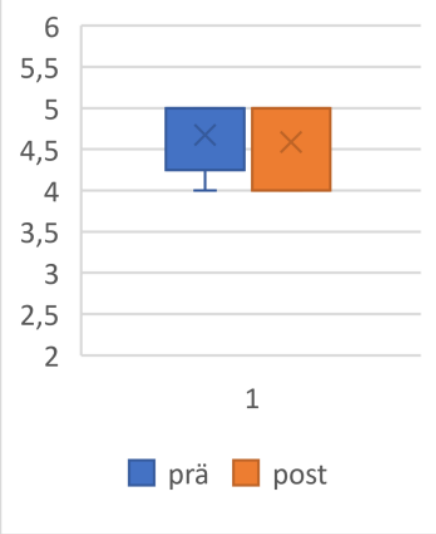

Abbildung 31: RULA linker Handgelenk- und Rotationswert

Beschriftung der Ordinate beginnt bei niedrigmöglichstem Punktwert (2) und endet beim Höchstwert (6) dieser Region.

4.1.2 Prä-Post-Auswertung der Medianwerte und Quartilsabstände - Oberarm, Unterarm, Handgelenk \& Rotation rechte Körperhälfte

Tabelle 4 zeigt die gebildeten Medianwerte und Quartilsabstände aller Probanden der RULA Scores für die rechte Körperhälfte. Statistisch lässt sich auch für die rechte Körperhälfte kein signifikanter Unterschied $(p \geq 0,05)$ zwischen den jeweils untersuchten Werten der beiden Messungen beschreiben. Die stat. Signifikanz des rechten Oberarms liegt bei $\mathrm{p}=0,366$. Im Prä-Post-Vergleich bleibt der Medianwert des rechten Oberarms (Abb. 32) unverändert bei einem Score von 2. Beide Werte erreichen nicht den definierten Schwellenwert von 5 und somit bleibt auch die ergonomische Risikoeinschätzung unverändert. Der Wert des unteren Quartils ist vor der Intervention bei 1,5 und verändert sich ebenfalls nicht im Postscore. Das obere Quartil liegt sowohl im Prä- als auch im Postwert bei einem Score von 3. Der Quartilsabstand bleibt im Prä-Post-Vergleich unverändert bei 0,5 .

Für den rechten Unterarm liegt die stat. Signifikanz bei $\mathrm{p}=0,366$. Der RULA Medianscore (Abb. 33) liegt vor dem Interventionszeitraum bei 2 und danach ebenfalls bei 2. Die ergonomische Risikoschwelle bei Score 3 wird jeweils nicht erreicht und die Risikobewertung bleibt unverändert. Das erste Quartil liegt bei einem Präscore von 2 und auch im Postscore hat sich mit 2 an der Lage nichts verändert. Der Wert des dritten Quartils erhöht 
sich geringfügig von 2 im Präscore zu 2,5 im Postscore. Der Interquatilsabstand erhöht sich von Prä- zu Postwert ebenfalls um 0,5.

Tabelle 4: RULA Scores - Median- und Quartilwerte Rechtsseitig (Right) Oberarm (Upper Arm), Unterarm (Lower Arm), Handgelenk \& Rotation (Wrist Position and Twist)

Fett Markierung: Score wird gesundheitlich riskant eingestuft

\begin{tabular}{|c|c|c|c|c|c|}
\hline \multicolumn{2}{|c|}{$\begin{array}{l}\text { RULA Right Up- } \\
\text { per Arm Position }\end{array}$} & \multicolumn{2}{|c|}{$\begin{array}{l}\text { RULA Right Lower } \\
\text { Arm Position }\end{array}$} & \multicolumn{2}{|c|}{$\begin{array}{l}\text { RULA Right Wri } \\
\text { Position and Twist }\end{array}$} \\
\hline prä & post & prä & post & prä & post \\
\hline 1,5 & 1,5 & 2 & 2 & 4 & 4 \\
\hline 2 & 2 & 2 & 2 & 4,5 & 4,5 \\
\hline 2 & 2 & 2 & 2,5 & 5 & 5 \\
\hline 0,5 & 0,5 & 0 & 0,5 & 1 & 1 \\
\hline
\end{tabular}

Für den rechten Handgelenks- \& Rotationswert liegt die stat. Signifikanz bei p=1,000. Der Medianwert (Abb. 34) hat sich vom Präwert 4,5 nach dem Interventionszeitraum nicht verändert und bleibt bei einem Postwert von 4,5. Der Medianwert der Probanden erreicht somit weder vor noch nach dem Interventionszeitraum die bei einem Wert von 5 definierte Risikoschwelle, bei einer unveränderten Risikoeinschätzung. Das erste Quartil liegt sowohl prä als auch post bei einem Wert von 4. Auch das dritte Quartil liegt im Präsowie Postscore gleich bei einem Wert von 5. Somit liegen jeweils für die Prä- und Postmessungen mindestens 25\% der Probanden bei einem Score $\geq 5$, deren ergonomische Arbeitsweise dadurch als gesundheitlich riskant eingestuft wird. Der Interquartilsabstand liegt im Präwert bei 1 und bleibt auch im Postwert unverändert bei 1 . 


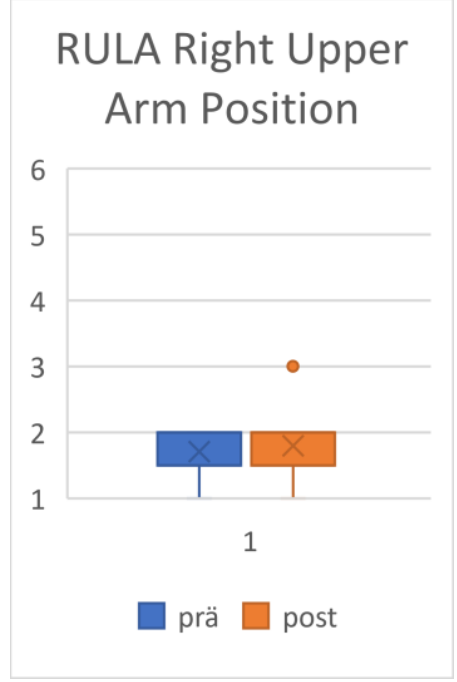

Abbildung 32: Rula rechter Oberarmwert

Beschriftung der Ordinate beginnt bei niedrigmöglichstem Punktwert (1) und endet beim Höchstwert (6) dieser Region.
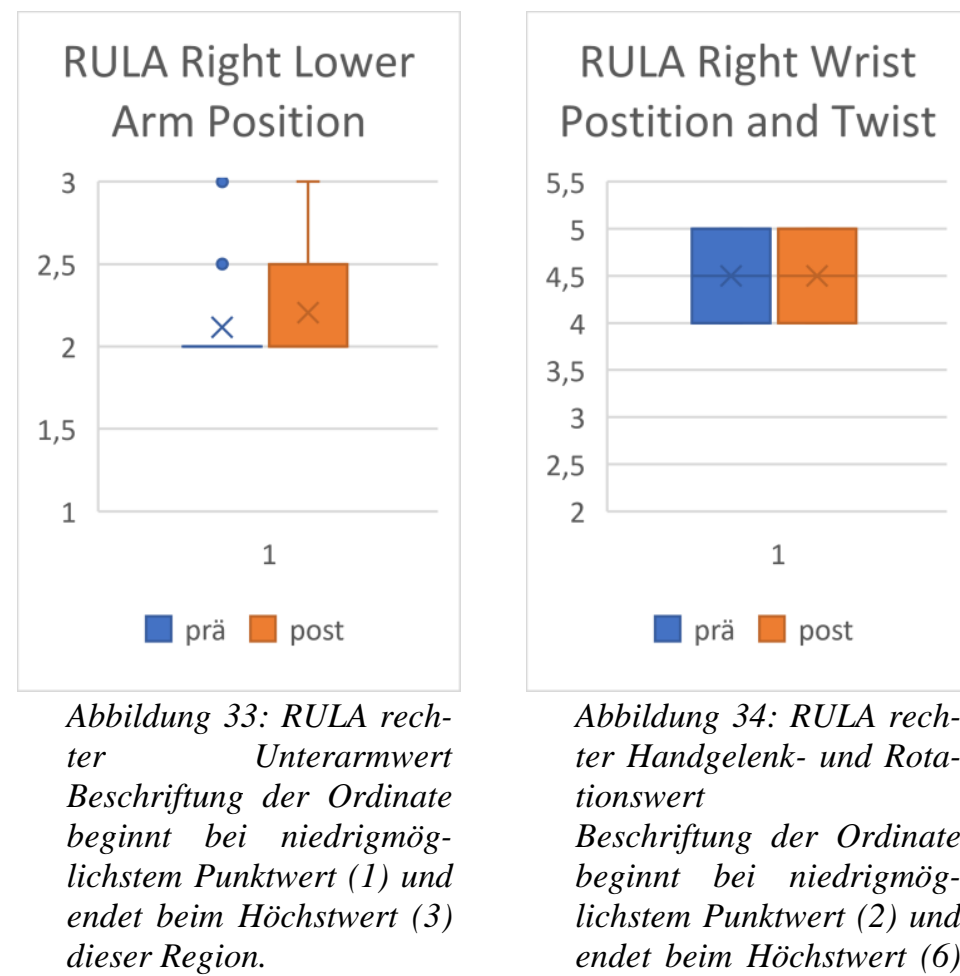

Abbildung 34: RULA rechter Handgelenk- und Rotationswert

Beschriftung der Ordinate beginnt bei niedrigmöglichstem Punktwert (2) und endet beim Höchstwert (6) dieser Region.

4.1.3 Prä-Post-Auswertung der Medianwerte und Quartilsabstände - Oberarm, Unterarm, Handgelenk \& Rotation im Seitenvergleich

Im Seitenvergleich von rechter und linker Körperhälfte fallen z.T. Abweichungen zwischen den jeweiligen Regionen auf. Exemplarisch werden im Folgenden die Medianwerte und Interquartilsabstände verglichen. Der statistische Vergleich der erhobenen Werte des rechten und linken Oberarms zeigt sowohl für die Prämessung einen signifikanten Unterschied $(\mathrm{p} \leq 0,01)$ als auch für die Postmessung $(\mathrm{p} \leq 0,05)$. Die stat. Signifikanz liegt bei $\mathrm{p}=0,007$ für die Prämessung und $\mathrm{p}=0,047$ für die Postmessung. Bei den Medianwerten fällt für die Oberarmregion eine um 1 Punkt im Präwert und um 0,5 Punkte im Postwert höhere Bepunktung für die rechte Körperhälfte auf, ohne dass sich eine unterschiedliche Risikobewertung ergeben würde. In der Unterarmregion zeigt der statistische Vergleich von rechts und links einen signifikanten Unterschied für die Prämessung ( $p \leq 0,05$ ), für die Postmessung liegt kein statistisch signifikanter Unterschied ( $\mathrm{p} \geq 0,05)$ vor. Der Signifikanzwert lag bei $p=0,028$ für die Prämessung und $p=0,158$ für die Postmessung. Deskriptiv zeigt sich, dass alle Medianwerte für die rechte und linke Unterarmregion prä und post einheitlich bei 2 liegen, außer dem Median der Postmessung für die linke Körperhälfte, dieser ist mit 2,5 um 0,5 Punkte höher bewertet. 
Für die Handgelenks und Rotationswerte lässt sich im Seitenvergleich kein signifikanter $(p \geq 0,05)$ Unterschied zwischen den jeweils untersuchten Werten der beiden Messungen beschreiben. Die statistische Signifikanz liegt während der Prämessung bei p=0,294 und während der Postmessung bei p=0,825. Deskriptiv liegen sowohl Prä- als auch Postwert der linken Körperhälfte mit einem Score von 5 um 0,5 Punkte höher als die jeweiligen Scores der rechten Körperhälfte. Dadurch erreicht der Wert auf der linken Körperhälfte die Risikoschwelle und die ergonomische Arbeitsweise wird linksseitig als gesundheitlich riskant bewertet während dies rechts nicht der Fall ist. Im direkten Vergleich sind 3/6 Medianwerte der linken Körperhälfte und 2/6 Medianwerte der rechten Körperhälfte geringfügig höher als auf der Gegenseite. Ein Wert ist gleich.

Die Interquartilsabstände der jeweiligen Oberarmregion sind bei allen Messungen bei 0,5 außer bei der Postmessung des linken Oberarms hier ist der Abstand mit 1 geringfügig höher. Im Vergleich der Quartilsabstände des linken und rechten Unterarms, ist der Quartilsabstand der Präwerte linksseitig um 1 Punkt größer und im Postwert linksseitig um 0,5 Punkte größer. Die Quartilsabstände für die Handgelenks- und Rotationswerte liegen prä und post alle bei 1 außer dem Abstand der linksseitigen Prämessung der geringfügig schmaler bei 0,5 liegt. Somit sind die Interquartilsabstände im direkten Vergleich 2/6-mal gleich, 1/6-mal rechtsseitig geringfügig breiter und 3/6-mal linksseitig geringfügig breiter.

4.1.4 Prä-Post-Auswertung der Medianwerte und Quartilsabstände - Nacken und Oberkörper

Tabelle 5 zeigt die gebildeten Medianwerte und Quartilsabstände aller Probanden der RULA Scores für die Nacken- und Oberkörperregion. Statistisch kann mit dem Wilcoxon-Matched-Pairs Test kein signifikanter $(\mathrm{p} \geq 0,05)$ Unterschied zwischen den untersuchten Werten der jeweiligen Messungen beschrieben werden. Der Signifikanzwert liegt bei $\mathrm{p}=0,527$. Der Medianwert der Nackenregion (Abb. 35) liegt im Präwert bei einem Score von 3 und bleibt auch im Postwert unverändert bei 3. Die ergonomische Risikobeurteilung verändert sich somit nicht und die Arbeitsweise wird prä und post nicht als gesundheitlich riskant eingestuft. Das erste Quartil liegt sowohl im Prä- als auch im Postscore bei einem Wert von 3. Das dritte Quartil liegt im Präwert bei 4 und somit erreich mindestens 25\% der Probanden einen Score größer gleich der ergonomischen Risiko- 
schwelle. Im Postwert liegt das dritte Quartil bei 3,5; somit wird die ergonomische Arbeitsweise nach der Intervention nicht mehr als gesundheitlich riskant eingestuft. Der Quartilsabstand verringert sich von 1 vor dem Interventionszeitraum auf 0,5 danach.

Tabelle 5: RULA Scores - Median- und Quartilwerte: Nacken (Neck) und Oberkörper (Trunk)

Fett Markierung: Score wird gesundheitlich riskant eingestuft

RULA Neckscore RULA Trunk Score

$\begin{array}{lllll} & \text { prä } & \text { post } & \text { prä } & \text { post } \\ \text { 1. Quartil } & 3 & 3 & 2 & 2 \\ \text { Median } & 3 & 3 & 2 & 2 \\ \text { 3. Quartil } & 4 & 3,5 & 2,5 & 3 \\ \text { Quartilsabstand } & 1 & 0,5 & 0,5 & 1\end{array}$

In der Oberkörperregion ist die stat. Signifikanz bei $\mathrm{p}=0,340$. Im Prä-Post-Vergleich der Medianwerte (Abb. 36), liegt der Score jeweils prä und post bei 2. Für beide Messungen wird die ergonomische Risikoschwelle somit nicht erreicht. Das untere Quartil der Region zeigt ebenfalls keine Abweichung der beiden Messungen und liegt jeweils bei einem Wert von 2. Das obere Quartil lag bei der Prämessung bei einem Wert von 2,5 und hat sich bei der Postmessung marginal um 0,5 Punkte auf 3 erhöht. Der Interquartilsabstand war vor dem Interventionszeitraum bei einem Abstand von 0,5 Punkten und hat sich nach dem Interventionszeitraum ebenfalls geringfügig um 0,5 Punkte auf einen Abstand von 1 erhöht. 


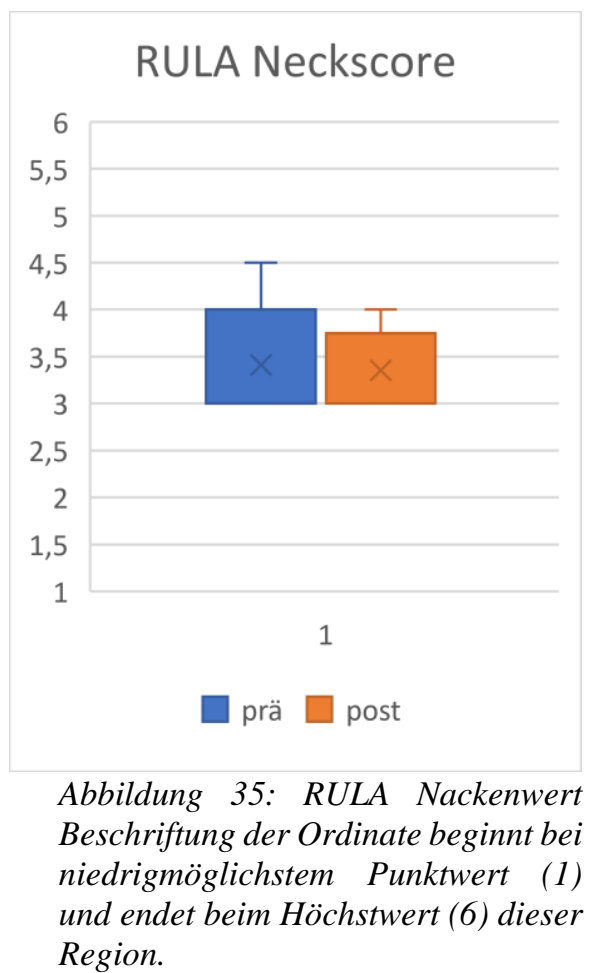

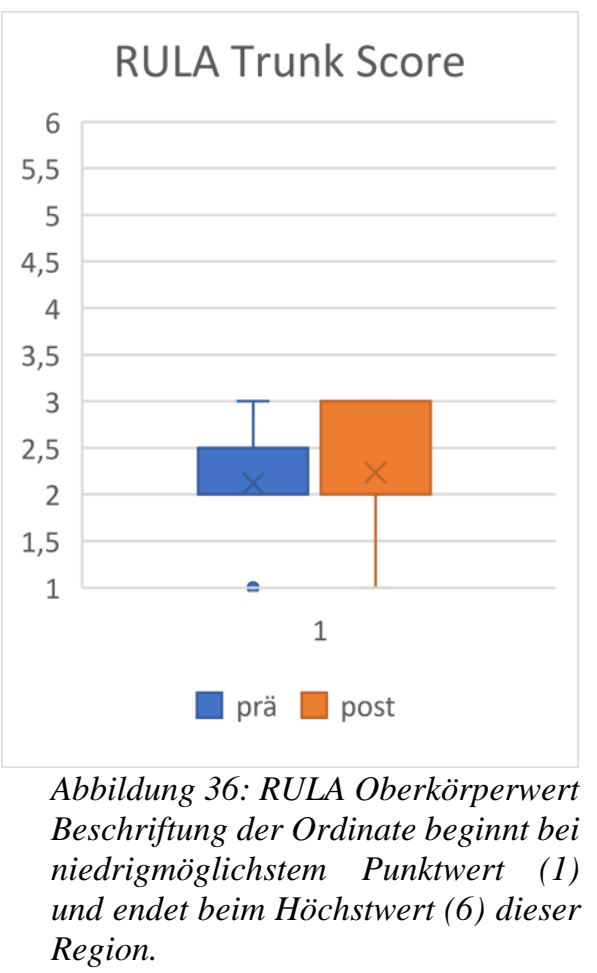

Region.

\subsubsection{Prä-Post-Auswertung der Medianwerte und Quartilsabstände - RULA Gesamtscore}

Tabelle 6 zeigt die gebildeten Medianwerte und Quartilsabstände aller Probanden der RULA Gesamtscores. Statistisch kann kein signifikanter $(\mathrm{p} \geq 0,05)$ Unterschied zwischen den untersuchten Werten der jeweiligen Messungen beschrieben werden. Der Signifikanzwert ist $\mathrm{p}=0,317$. Die Prä- und Postwerte für den Median der RULA Gesamtscores (Abb. 37) haben sich im Vergleich vor und nach dem Interventionszeitraum nicht verändert und liegen jeweils bei 7. Die Probanden erreichen somit den erzielbaren Höchstwert und ebenfalls die ergonomische Risikoschwelle. Die ergonomische Arbeitsweise wird als gesundheitlich riskant eingestuft. Die aus dem RULA Arbeitsbogen abgeleitete Handelsempfehlung sieht eine weitere Untersuchung und sofort notwendige Veränderung der Arbeitsweise vor. Das untere Quartil hat sich ebenfalls nicht verändert und liegt prä sowie post unverändert bei 7. Das obere Quartil zeigt keine Veränderung im Prä-Post-Vergleich und liegt jeweils beim Maximalwert von 7. Somit erreichen mindestens 75\% der Probanden einen RULA Gesamtscore von 7. Die Abbildung 34 zeigt, dass es im Prä-Post-Vergleich unter den Probanden bei der Postmessung einen Ausreißer mit einem RULA Gesamtscore von 6,5 gibt. Dieser Proband erreicht die ergonomische Risikoschwelle nicht. Die Gesamtscore liegt zwischen 5 und 6 die abgeleitete Handlungsempfehlung aus dem 
RULA Arbeitsbogen lautet für diesen Probanden: weitere Untersuchung sowie eine baldige Veränderung der ergonomischen Arbeitsweise vor.

Tabelle 6: RULA Scores - Median- und Quartilwerte: Gesamtwerte

Fett Markierung: Score wird gesundheitlich riskant eingestuft

\begin{tabular}{lll} 
& $\begin{array}{l}\text { RULA } \\
\text { score }\end{array}$ & Gesamt- \\
prä & post \\
\hline 1. Quartil & $\mathbf{7}$ & $\mathbf{7}$ \\
Median & $\mathbf{7}$ & $\mathbf{7}$ \\
3. Quartil & $\mathbf{7}$ & $\mathbf{7}$ \\
Quartilsabstand & 0 & 0
\end{tabular}

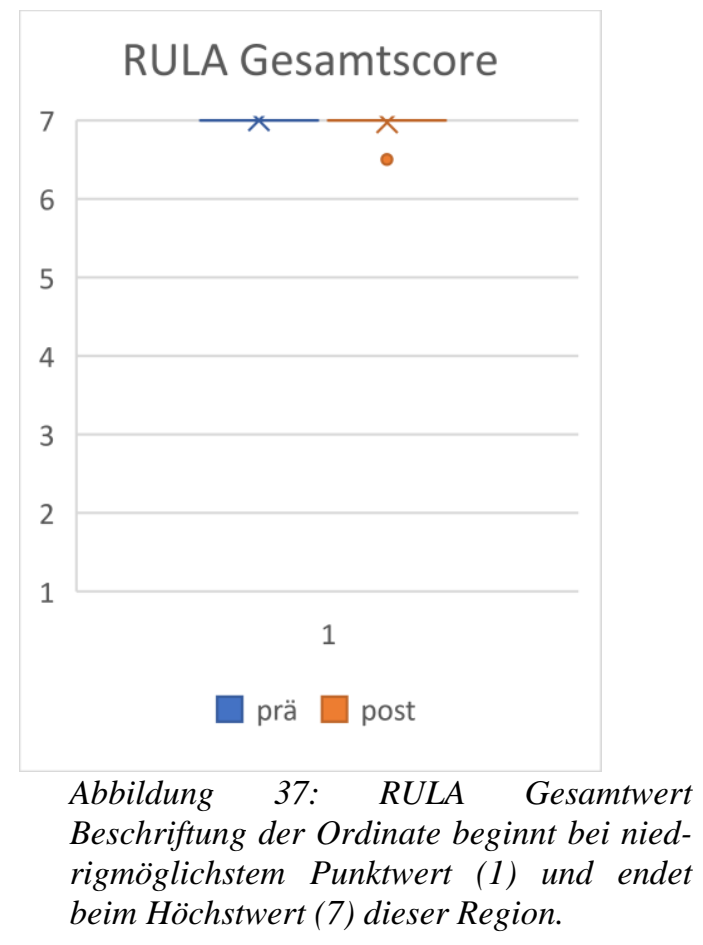

4.1.6 Prä-Post-Auswertung der Medianwerte und Quartilsabstände - Berufsgruppenvergleich

Im folgenden Kapitel werden die bereits angeführten Ergebnisse der Prä-Post-Auswertung der Medianwerte und Quartilsabstände für die gesamte Probandenkohorte verglichen mit den Ergebnissen der Prä-Post-Auswertung für die isolierte Berufsgruppe der Zahnärzte. Statistisch können keine signifikanten Unterschiede ( $\mathrm{p} \geq 0,05)$ zwischen den Gruppen beschrieben werden. Es lassen sich vereinzelte Varianzen deskriptiv beschreiben.

Tabelle 7 zeigt die Medianwerte sowie den Interquantilsabstand der Zahnärzte für die linke Körperhälfte. Im Vergleich der linken Oberarmregion zwischen allen Probanden und der Gruppe der ZÄ lässt sich für Medianwert kein Unterschied beschreiben und somit bleibt auch die Risikobeurteilung gleich. Der Interquatilsabstand der Postmessung ist in der Gruppe der Z $\overparen{A}$ mit 0,75 marginal kleiner als der Abstand der Gesamtkohorte von 1. 
Bei Betrachtung des linken Unterarms für beide Gruppen lässt sich beim Medianwert in der Prämessung ein mit 2,5 geringfügig höherer Score für die ZÄ im Vergleich zur Gesamtgruppe mit einem Score von 2 beschreiben. Die Risikobeurteilung ändert sich dadurch nicht. Der Interquartilsabstand liegt für alle betrachteten Werte bei 1, zwischen den Gruppen zeigt sich demnach kein Unterschied.

Tabelle 7: RULA Scores Z̈̈ - Median- und Quartilsabstand Linksseitig (Left) Oberarm (Upper Arm), Unterarm (Lower Arm), Handgelenk \& Rotation (Wrist Position and Twist)

Fett Markierung: Score wird gesundheitlich riskant eingestuft

\begin{tabular}{|c|c|c|c|c|c|c|}
\hline & \multicolumn{2}{|c|}{$\begin{array}{l}\text { RULA Left Upper } \\
\text { Arm Position }\end{array}$} & \multicolumn{2}{|c|}{$\begin{array}{l}\text { RULA Left Lower } \\
\text { Arm Position }\end{array}$} & \multicolumn{2}{|c|}{$\begin{array}{l}\text { RULA Left Wrist } \\
\text { Postion and Twist }\end{array}$} \\
\hline & prä & post & prä & post & prä & post \\
\hline Median & 1 & 1,5 & 2,5 & 2,5 & 5 & 5 \\
\hline Quartilsabstand & 0,5 & 0,75 & 1 & 1 & 0,5 & 0,5 \\
\hline
\end{tabular}

Der linksseitige Vergleich der Handgelenks- \& Rotationswerte ergibt für den Medianwert zwischen den Gruppen kein Unterschied. Der Wert ist auch für die isolierte Gruppe der ZÄ Prä- und Post bei 5 und somit wird die ergonomische Arbeitsweise als gesundheitlich riskant eingestuft. Der Interquantilsabstand für die Prämessung beider Gruppen ist mit 0,5 identisch. In der Postmessung gibt es eine kleine Abweichung zwischen 1 für die gesamte Kohorte und 0,5 für die Z $\ddot{A}$.

Tabelle 8 zeigt die die Medianwerte sowie den Interquartilsabstand rechtsseitig. Für den rechten Oberarm ist der Medianwert von 2 für beide Gruppen und beide Messungen identisch. Demnach verändert sich die ergonomische Arbeitsweise nicht. Der Interquartilsabstand liegt in der Prä- und Postmessung für die ZÄ bei jeweils 0,25 und ist somit marginal geringer als in der Gesamtgruppe. Hier liegt er für beide Messungen bei jeweils 0,5.

Der Vergleich des Medianwerts des rechten Unterarmes zwischen den beiden Gruppen so ist kein Unterschied erkennbar. Der Wert ist in beiden Gruppen für beide Messungen bei 2. Die Risikobeurteilung bleibt somit gleich. Für den Interquartilsabstand lässt sich nur in der Postmessung der beiden Gruppen ein geringfügiger Unterschied beschreiben. Der Score für die Gesamtgruppe ist mit 0,5 etwas höher als bei den ZÄ mit 0 .

Die Werte für Handgelenk \& Rotation rechtsseitig zeigen bei den Medianwerten nur für die Prämessung eine kleine Varianz. Der Medianwert ist in der Gruppe aller Probanden 0,5 Punkte höher als der Wert von 4 bei den ZÄ. Das gesundheitliche Risiko der ergonomischen Arbeitshaltung wird weiterhin gleich eingeschätzt. Der Interquartilsabstand für beide Gruppen und alle Messungen liegt bei 1 und zeigt demnach keinen Unterschied. 
Tabelle 8: RULA Scores Z̈̈ - Median und Quartilsabstand Rechtsseitig (Right) Oberarm (Upper Arm), Unterarm (Lower Arm), Handgelenk \& Rotation (Wrist Position and Twist)

Fett Markierung: Score wird gesundheitlich riskant eingestuft

\begin{tabular}{|c|c|c|c|c|c|c|}
\hline & \multirow{2}{*}{\multicolumn{2}{|c|}{$\begin{array}{l}\text { RULA Right Upper } \\
\text { Arm Position }\end{array}$}} & \multirow{2}{*}{\multicolumn{2}{|c|}{$\begin{array}{l}\text { RULA Right } \\
\text { Lower Arm Posi- } \\
\text { tion }\end{array}$}} & \multicolumn{2}{|c|}{ RULA Right Wrist } \\
\hline & & & & & \multicolumn{2}{|c|}{$\begin{array}{l}\text { Postition and } \\
\text { Twist }\end{array}$} \\
\hline & prä & post & prä & post & prä & post \\
\hline Median & 2 & 2 & 2 & 2 & 4 & 4,5 \\
\hline Quantilsabstand & 0,25 & 0,25 & 0 & 0 & 1 & 1 \\
\hline
\end{tabular}

Tabelle 9 zeigt die die Medianwerte sowie den Interquartilsabstand für Nacken und Oberkörper. Für den Vergleich der Medianwerte in der Nackenregion ist dieser in beiden Gruppen sowie für Prä- und Postmessung bei 3. Es lässt sich folglich kein Unterschied beschreiben und auch das ergonomische Risiko bleibt unverändert. Der Interquartilsabstand ist bei beiden Gruppen prä bei 1 und post bei 0,5 und zeigt somit keinen Unterschied.

Wird die Oberkörperregion betrachtet liegt der jeweilige Score für die Prämessung beider Gruppen bei 2. In der Postmessung lässt sich ein Unterschied zwischen einem Score von 2 in der Gesamtgruppe und 3 in der Z beurteilung ändert sich dadurch nicht. Die Interquartilsabstände sind bei beiden Gruppen in der Prämessung bei jeweils 0,5 Punkten, in der Postmessung bei 1 Punkt und somit identisch.

Tabelle 9: Tabelle 5: RULA Scores Z̈̈ - Median und Quartilsabstand: Nacken (Neck) und Oberkörper (Trunk)

Fett Markierung: Score wird gesundheitlich riskant eingestuft

\begin{tabular}{lllll} 
& \multicolumn{2}{l}{ RULA Neckscore } & \multicolumn{2}{l}{ RULA Trunk Score } \\
& prä & post & prä & post \\
Median & 3 & 3 & 2 & 3 \\
Quantilsabstand & 1 & 0,5 & 0,5 & 1
\end{tabular}

Tabelle 10 zeigt den RULA Gesamtscore der Zahnärztegruppe. Im Vergleich der Medianscores zwischen den Z̈̈ und der gesamten Probandenkohorte sind die Werte für beide Messungen und beide Berufsgruppen jeweils beim Höchstwert 7. Die ergonomische Arbeitsweise wird demnach auch für die isolierte Gruppe der Z $\overparen{A}$ als gesundheitlich riskant eingestuft. Der Interquartilsabstand ist bei der Gesamtgruppe sowie bei den Zahnärzten bei beiden Messungen bei 0 . Es lässt sich folglich kein Unterschied beschreiben. 
Tabelle 10: RULA Scores Z̈̈ - Median und Quartilsabstand: Gesamtwerte

Fett Markierung: Score wird gesundheitlich riskant eingestuft

RULA Gesamtscore

$\begin{array}{lll} & \text { prä } & \text { post } \\ \text { Median } & 7 & 7 \\ \text { Quantilsabstand } & 0 & 0\end{array}$

\subsection{Prä-Post-Auswertung der Bewertungsparameter nach relativer zeitlicher Ver- teilung}

Die mit RULA bestimmten Werte für den Gesamtscore und die einzelnen Körperregionen errechnen sich aus der relativen zeitlichen Verteilung der Bewertungsparameter (Scores). Der Unterschied zwischen der relativen Zeitverteilung der Scores wird im Prä-Post-Vergleich in diesem Kapitel dargestellt. In der statistischen Auswertung wurden keine signifikanten Unterschiede $(\mathrm{p} \geq 0,05)$ im Prä-Post-Vergleich der untersuchten Parameter festgestellt. Es lassen sich allerdings deskriptive Veränderung beschreiben.

4.2.1 Prä-Post-Auswertung der Bewertungsparameter nach relativer zeitlicher Verteilung - Oberarm linksseitig

Tabelle 7 zeigt die relative zeitliche Verteilung der Bewertungsparameter in Prozent für den linken Oberarm im Prä-Post-Vergleich. Der Signifikanzwert liegt bei $\mathrm{p}=0,981$. Sowohl für die Prä- als auch die Postmessung wurde jeweils 0\% in den Scores 5 und 6 verbracht.

\begin{tabular}{|c|c|c|c|c|c|c|}
\hline \multirow{2}{*}{ Spalte1 } & RULA & RULA & RULA & RULA & RULA & \\
\hline & Score 1 & Score 2 & Score 3 & Score 4 & Score 5 & Score 6 \\
\hline prä & $50,79 \%$ & $47,57 \%$ & $1,64 \%$ & $0,00 \%$ & $0,00 \%$ & $0,00 \%$ \\
\hline post & $40,34 \%$ & $56,78 \%$ & $2,86 \%$ & $0,02 \%$ & $\mathbf{0 , 0 0 \%}$ & $0,00 \%$ \\
\hline
\end{tabular}

Folglich verbrachten die Probanden bei beiden Messungen keine Zeit im als gesundheitlich riskant eingestuften Bewertungsbereich (Abb. 38). Die Zeit, die im RULA Score 4 verbracht wurde, ändert sich marginal von prä $0 \%$ auf post $0,02 \%$. Die relative zeitliche 
Verteilung im RULA Score 3 erhöht sich geringfügig von 1,64\% in der Prämessung auf 2,86\% in der Postmessung. Im RULA Score 2 verbrachten die Probanden während der Prämessung 47,57\% der Zeit und in der Postmessung 9,21\% mehr mit 56,78\%. Der relative Zeitanteil im Score 1 war in der Prämessung bei 50,79\% und verringerte sich in der Postmessung auf 40,34\%.

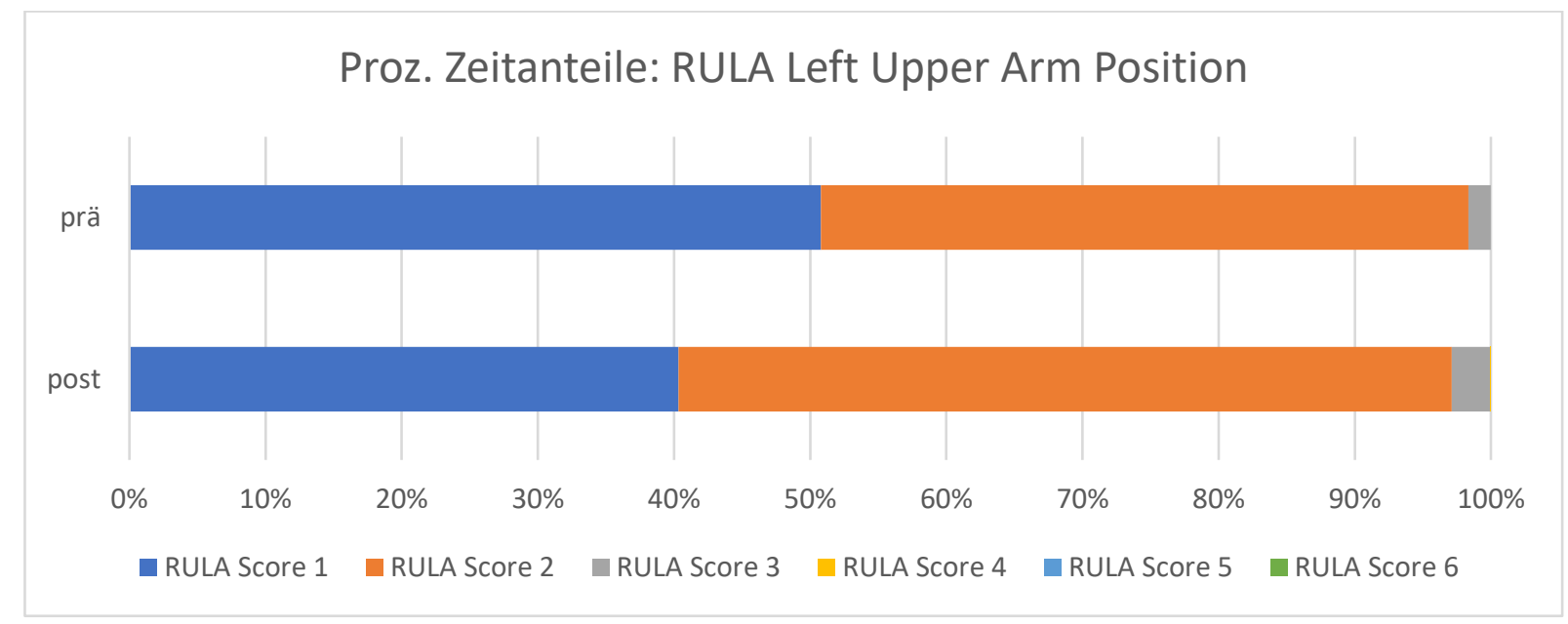

Abbildung 38: Proz. Zeitanteile: RULA Oberarm (Upper Arm) linksseitig

4.2.2 Prä-Post-Auswertung der Bewertungsparameter nach relativer zeitlicher Verteilung - Unterarm linksseitig

Tabelle 8 zeigt die relative zeitliche Verteilung der Bewertungsparameter in Prozent für den linken Unterarm im Prä-Post-Vergleich. Die stat. Signifikanz liegt bei p=0,981. Im als gesundheitlich riskant eingestuften RULA Score 3 verbringen während der Prämessung die Probanden 42,24\% der gemessenen Zeit.

Tabelle 12: Prozentuelle Zeitanteile: RULA Unterarm (Lower Arm) linksseitig

Fett Markierung: Score wird gesundheitlich riskant eingestuft

$\begin{array}{llll} & \text { RULA } & \text { RULA } & \text { RULA } \\ \text { prä } & \text { Score 1 } & \text { Score 2 } & \text { Score 3 } \\ \text { post } & 2,56 \% & 55,20 \% & \mathbf{4 2 , 2 4 \%} \\ & 2,91 \% & 46,31 \% & \mathbf{5 0 , 7 8 \%}\end{array}$

Dieser Wert erhöht sich in der Postmessung auf 50,78\%. Die Probanden verbringen in der Postmessung geringfügig mehr Zeit in einer ergonomisch riskanten Arbeitshaltung (Abb. 39).

Im Score 2 verbrachten die Probanden vor der Trainingsintervention 55,2\% der Zeit, nach der Intervention geringfügig weniger mit 46,31\% der Zeit. Für den RULA Score 1 lässt 
sich eine marginale Veränderung zwischen 2,56\% in der Prämessung und 2,91\% in der Postmessung ausmachen.

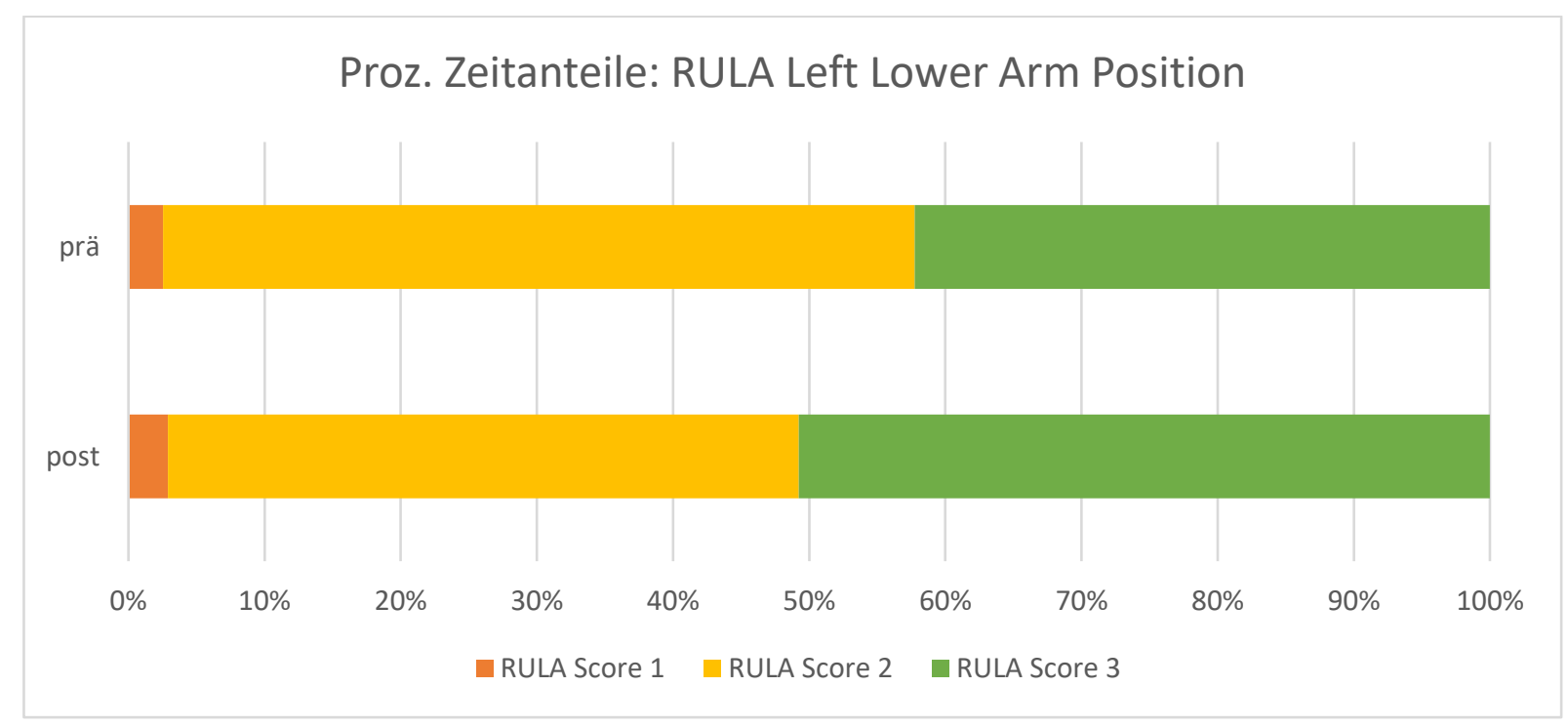

Abbildung 39: Proz. Zeitanteile: RULA Unterarm (Lower Arm) linksseitig

4.2.3 Prä-Post-Auswertung der Bewertungsparameter nach relativer zeitlicher Verteilung - Handgelenk \& Rotation linksseitig

Tabelle 9 zeigt die relative zeitliche Verteilung der Bewertungsparameter in Prozent für Handgelenk- und Rotation auf der linken Seite im Prä-Post-Vergleich. Der stat. Signifikanzwert liegt bei $\mathrm{p}=0,619$. Weder vor noch nach dem Interventionszeitraum wurde bei den Probanden der Höchstwert RULA Score 6 gemessen; die jeweiligen Werte waren somit bei $0 \%$. Im als Risikoschwelle definierten Score 5 verbrachten die Probanden in der Prämessung 62,22\% der Zeit und in der Postmessung 69,18\% der Zeit. Somit erhöhte sich die Zeit in einer gesundheitlich riskanten Arbeitsweise geringfügig um 6,96\%.

Tabelle 13: Prozentuelle Zeitanteile: RULA Handgelenk \& Rotation (Wrist Position and Twist) linksseitig Fett Markierung: Score wird gesundheitlich riskant eingestuft

$\begin{array}{lllllll} & \text { RULA } & \text { RULA } & \text { RULA } & \text { RULA } & \text { RULA } & \text { RULA } \\ \text { prä } & \text { Score } 1 & \text { Score } 2 & \text { Score 3 } & \text { Score 4 } & \text { Score 5 } & \text { Score 6 } \\ \text { post } & 0,00 \% & 0,21 \% & 1,87 \% & 35,70 \% & \mathbf{6 2 , 2 2 \%} & \mathbf{0 , 0 0 \%} \\ & 0,00 \% & 0,21 \% & 1,93 \% & 28,68 \% & \mathbf{6 9 , 1 8 \%} & \mathbf{0 , 0 0 \%}\end{array}$

Der RULA Score 4 verringerte sich geringfügig von 35,7\% in der Prämessung auf 28,68\% in der Postmessung (Abb. 40). Im RULA Score 3 kann eine marginale Veränderung zwischen prä $1,87 \%$ und post $1,93 \%$ der Messzeit beschrieben werden. Die Werte für 
RULA Score 2 sind mit 0,21\% für beide Messungen identische geblieben. Die Probanden arbeiteten weder vor noch nach der Intervention im RULA Score 1, der Wert ist jeweils bei $0 \%$.

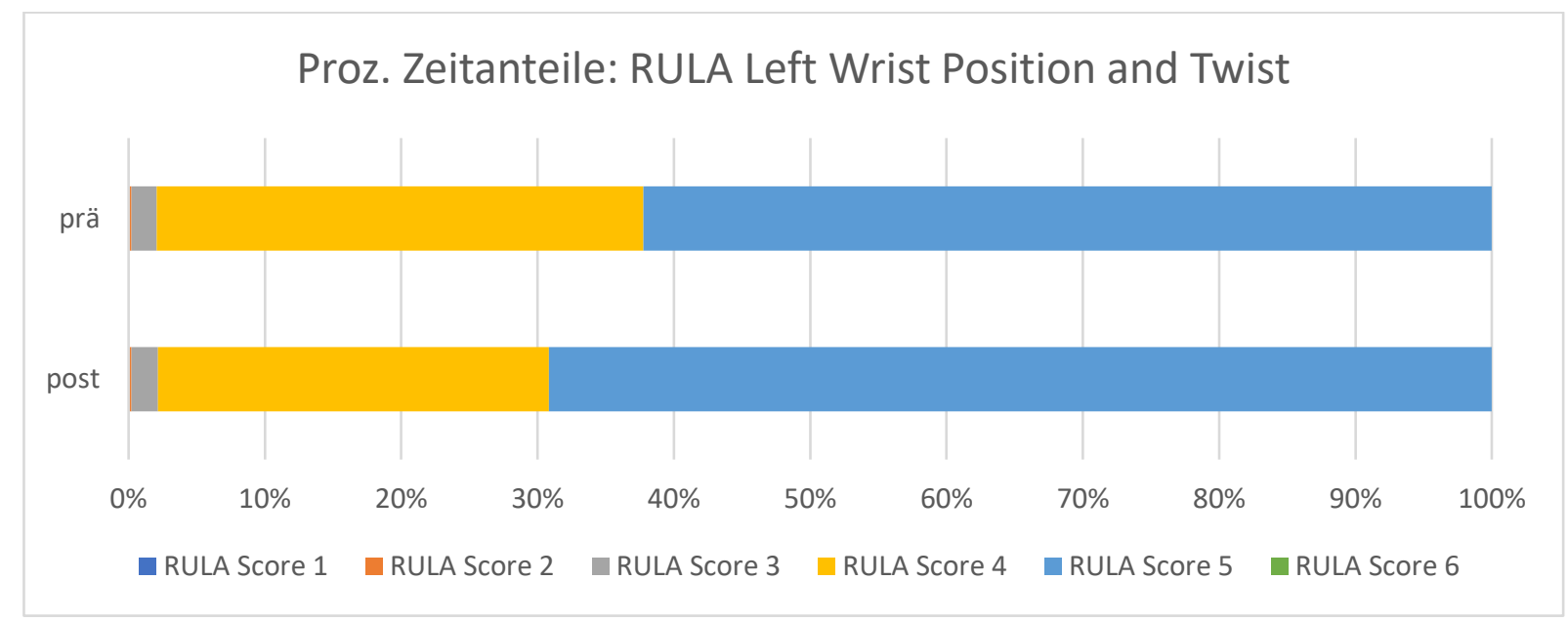

Abbildung 40: Prozentuelle Zeitanteile: RULA Handgelenk \& Rotation (Wrist Position and Twist) linksseitig

4.2.4 Prä-Post-Auswertung der Bewertungsparameter nach relativer zeitlicher Verteilung - Oberarm rechtsseitig

Tabelle 10 zeigt die relative zeitliche Verteilung der Bewertungsparameter in Prozent für Handgelenk- und Rotation auf der rechten Seite im Prä-Post-Vergleich. Statistisch liegt der Signifikanzwert bei $\mathrm{p}=0,435$. In den für die ergonomische Risikobewertung als riskant eingestuften RULA Scores 5 und 6 liegt der Wert bei beiden Messungen bei 0\%. Es wurde somit in der rechten Oberarmregion keine Zeit in gesundheitlich riskanter Arbeitshaltung verbracht.

Tabelle 14: Prozentuelle Zeitanteile: RULA Oberarm (Upper Arm) rechtsseitig Fett Markierung: Score wird gesundheitlich riskant eingestuft

$\begin{array}{lllllll} & \text { RULA } & \text { RULA } & \text { RULA } & \text { RULA } & \text { RULA } & \text { RULA } \\ \text { prä } & \text { Score } 1 & \text { Score 2 } & \text { Score 3 } & \text { Score 4 } & \text { Score 5 } & \text { Score 6 } \\ \text { post } & 40,17 \% & 53,31 \% & 4,93 \% & 1,59 \% & \mathbf{0 , 0 0 \%} & \mathbf{0 , 0 0 \%} \\ & 32,92 \% & 57,38 \% & 8,17 \% & 1,53 \% & \mathbf{0 , 0 0 \%} & \mathbf{0 , 0 0 \%}\end{array}$

Die prozentuellen Zeitanteile im RULA Score 4 haben sich nicht marginal verändert, von 1,59\% in der Prämessung auf 1,53\% in der Postmessung. Die im RULA Score 3 verbrachte Zeit hat sich von prä 4,93\% auf post $8,17 \%$ geringfügig erhöht. 53,31\% der Zeit 
arbeiteten die Probanden in der Prämessung in RULA Score 2, dieser Wert erhöhte sich geringfügig auf 57,38\% in der Postmessung. Die prozentuellen Zeitanteile im RULA Score 1 verringerten sich von 40,17\% in der Prämessung auf 32,92\% in der Postmessung (Abb. 41).

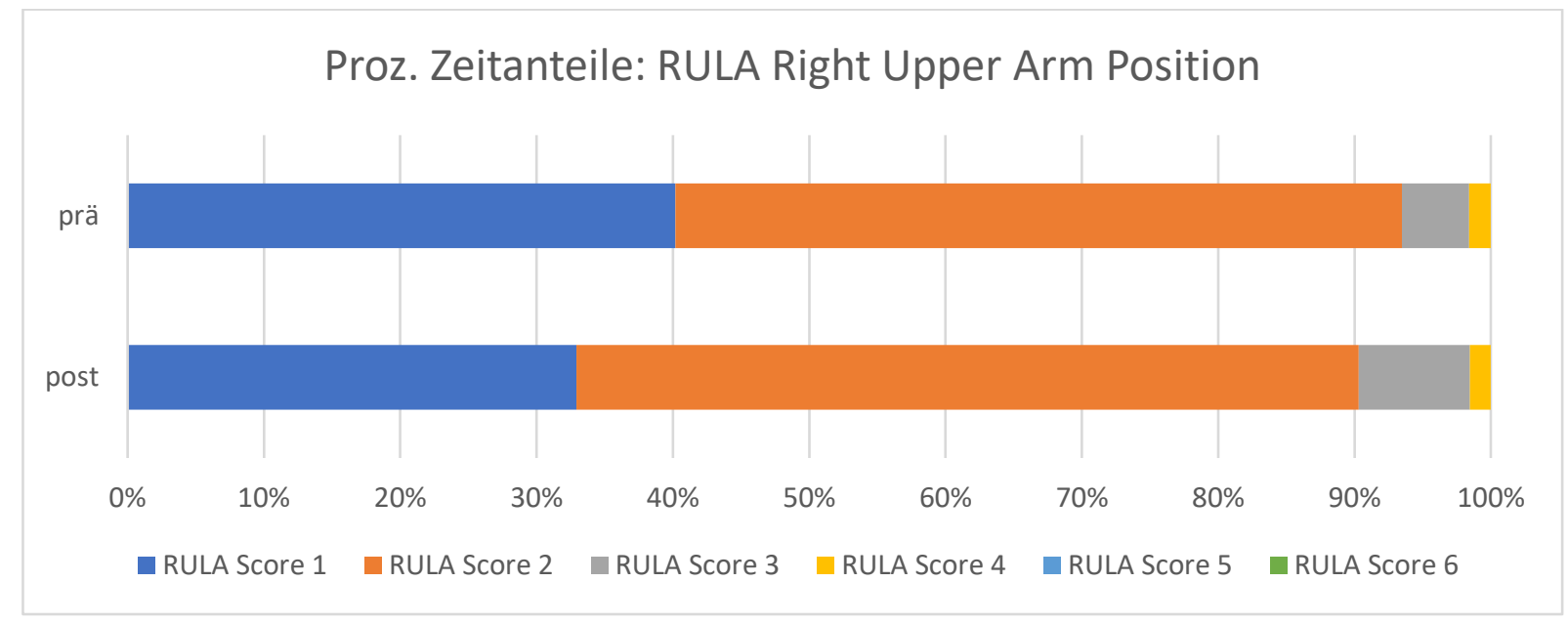

Abbildung 41: Proz. Zeitanteile: RULA Oberarm (Upper Arm) rechtsseitig

4.2.5 Prä-Post-Auswertung der Bewertungsparameter nach relativer zeitlicher Verteilung - Unterarm rechtsseitig

Die Tabelle 11 zeigt die relative zeitliche Verteilung der Bewertungsparameter in Prozent für den Unterarm auf der rechten Seite im Prä-Post-Vergleich. Die stat. Signifikanz liegt bei p=0,163. Im RULA Score 3 verbrachten die Probanden vor der Krafttrainingsintervention 20,45\% und danach 35,42\% der Zeit. Die Probanden arbeiteten in der Postmessung 14,97\% mehr in einer gesundheitlich riskanten Arbeitshaltung.

Tabelle 15: Prozentuelle Zeitanteile: RULA Unterarm (Lower Arm) rechtsseitig

Fett Markierung: Score wird gesundheitlich riskant eingestuft

$\begin{array}{llll} & \text { RULA } & \text { RULA } & \text { RULA } \\ & \text { Score } 1 & \text { Score 2 } & \text { Score 3 } \\ \text { prä } & 3,72 \% & 75,83 \% & \mathbf{2 0 , 4 5 \%} \\ \text { post } & 2,13 \% & 62,45 \% & \mathbf{3 5 , 4 2 \%}\end{array}$

Im RULA Score 2 lagen die prozentuellen Zeitanteile in der Prämessung bei 75,83\% und in der Postmessung geringfügig weniger mit 62,45\% der Zeit. Die Veränderung im Prä-Post- 
Vergleich für RULA Score 1 sind margnial mit 3,72\% vor und 2,13\% nach dem Trainingszeitraum (Abb. 42).

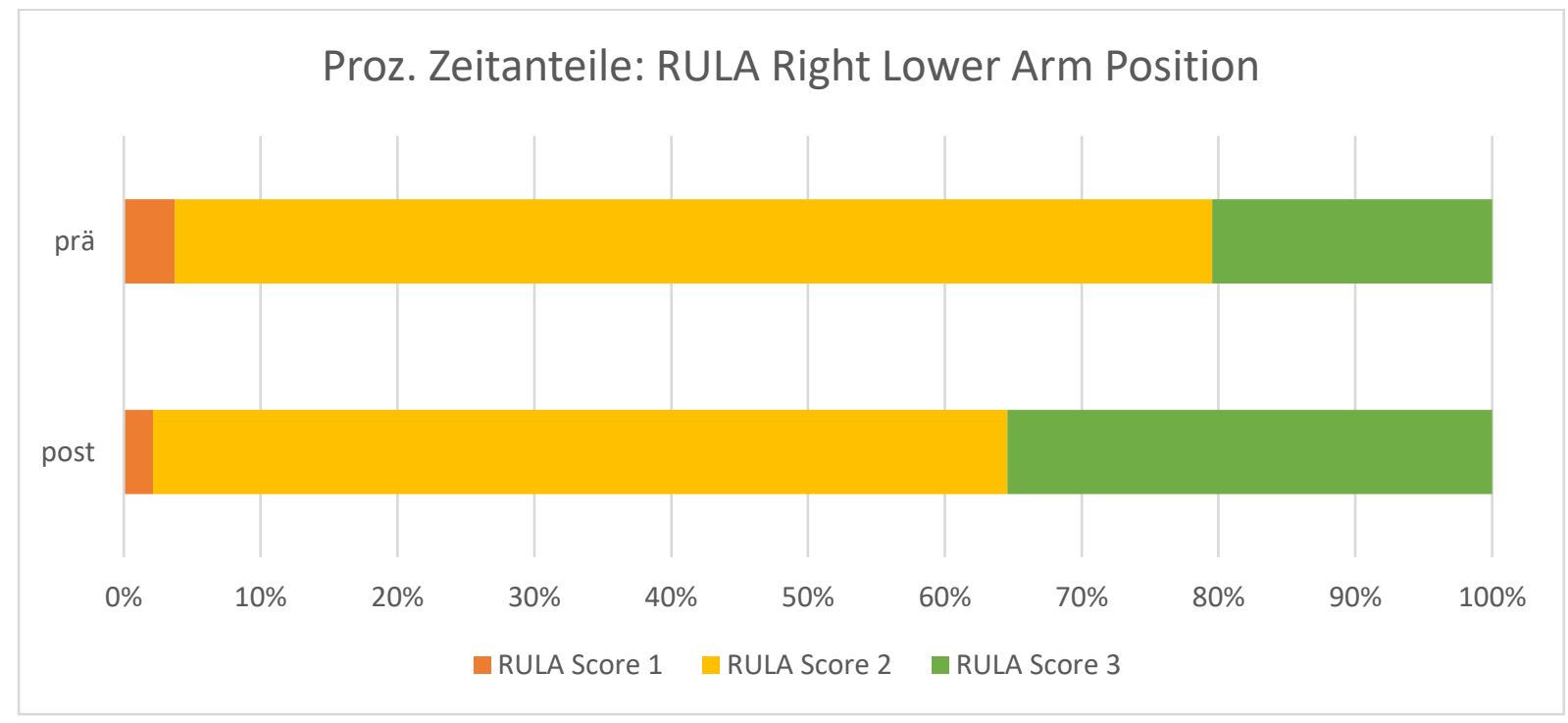

Abbildung 42: Proz. Zeitanteile: RULA Unterarm (Lower Arm) rechtsseitig

4.2.6 Prä-Post-Auswertung der Bewertungsparameter nach relativer zeitlicher Verteilung - Handgelenk \& Rotation rechtsseitig

Tabelle 12 zeigt die relative zeitliche Verteilung der Bewertungsparameter in Prozent für den Unterarm auf der rechten Seite im Prä-Post-Vergleich. Die stat. Signifikanz liegt bei p=0,188. Im RULA Score 6 arbeiteten die Probanden in 0\% der Zeit während Prä- und Postmessung. Der prozentuelle Anteil, den die Probanden im RULA Score 5 und somit definierten gesundheitlich riskanten Wertebereich verbrachten, lag während der Prämessung bei 45,17\% und während der Postmessung geringfügig höher bei 52,87\%.

Tabelle 16: Prozentuelle Zeitanteile: RULA Handgelenk \& Rotation (Wrist Position and Twist) rechtsseitig Fett Markierung: Score wird gesundheitlich riskant eingestuft

$\begin{array}{lllllll}\text { Spalte1 } & \text { RULA } & \text { RULA } & \text { RULA } & \text { RULA } & \text { RULA } & \text { RULA } \\ & \text { Score } 1 & \text { Score } 2 & \text { Score } 3 & \text { Score } 4 & \text { Score 5 } & \text { Score 6 } \\ \text { prä } & 0,00 \% & 0,72 \% & 3,44 \% & 50,67 \% & \mathbf{4 5 , 1 7 \%} & \mathbf{0 , 0 0 \%} \\ \text { post } & 0,00 \% & 0,37 \% & 2,18 \% & 44,57 \% & \mathbf{5 2 , 8 7 \%} & \mathbf{0 , 0 0 \%}\end{array}$

Der prozentuale Anteil des RULA Score 4 war prä bei 50,67\% und post etwas geringer bei 44,57\%. Im RULA Score 3 lassen sich nur marginale Veränderungen beschreiben zwischen $3,44 \%$ in der Prämessung und $2,18 \%$ in der Postmessung. Auch im RULA 
Score 2 verringern sich die Anteile sehr geringfügig mit $0,72 \%$ in der ersten und $0,37 \%$ in der zweiten Messung (Abb. 43).

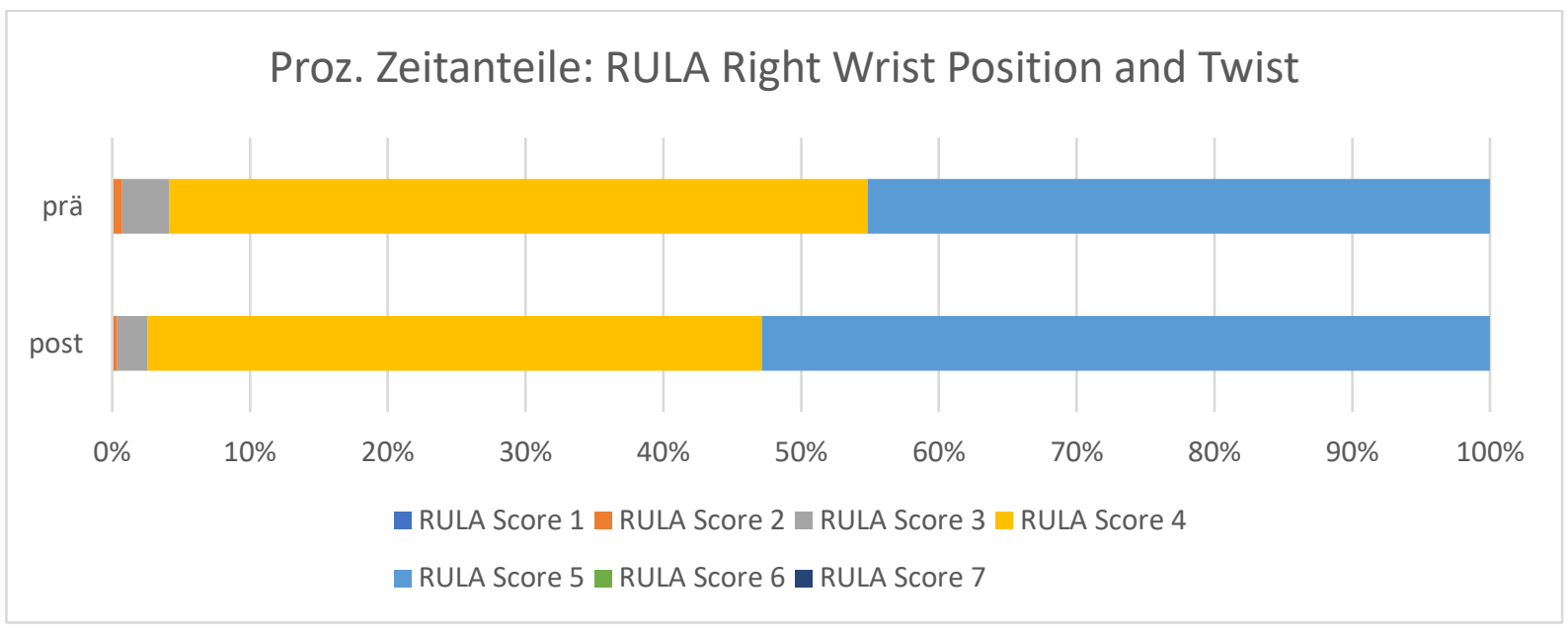

Abbildung 43: Proz. Zeitanteile: RULA Handgelenk \& Rotation (Wrist Position and Twist) rechtsseitig

4.2.7 Prä-Post-Auswertung der Bewertungsparameter nach relativer zeitlicher Verteilung - Nacken

Die Tabelle 13 zeigt die relative zeitliche Verteilung der Bewertungsparameter in Prozent für den Nacken im Prä-Post-Vergleich. Der Signifikanzwert liegt bei $\mathrm{p}=0,527$. 0\% der Zeit verbrachten die Probanden während der Prä- und Postmessung im höchsten Bewertungsparameter RULA Score 6. Die prozentualen Zeitanteile im gesundheitlich riskanten Score 5 verringerten sich geringfügig von 6,92\% in der Prämessung auf 2,94\% in der Postmessung. Die beschreibbare Veränderung in dem als Risikoschwelle definierten RULA Score 5 ist mit 37,17\% vor und 37,44\% nach der Trainingsintervention nur marginal.

Tabelle 17: Prozentuelle Zeitanteile: RULA Nacken (Neck)

Fett Markierung: Score wird gesundheitlich riskant eingestuft

$\begin{array}{lllllll} & \text { RULA } & \text { RULA } & \text { RULA } & \text { RULA } & \text { RULA } & \text { RULA } \\ & \text { Score } 1 & \text { Score } 2 & \text { Score 3 } & \text { Score 4 } & \text { Score 5 } & \text { Score 6 } \\ \text { prä } & 0,00 \% & 0,08 \% & 55,82 \% & \mathbf{3 7 , 1 7 \%} & \mathbf{6 , 9 2 \%} & \mathbf{0 , 0 0 \%} \\ \text { post } & 0,00 \% & 0,00 \% & 59,62 \% & \mathbf{3 7 , 4 4 \%} & \mathbf{2 , 9 4 \%} & \mathbf{0 , 0 0 \%}\end{array}$

Im RULA Score 3 verbrachten die Probanden prä 55,82\% und post ein wenig mehr mit 59,62\% der Zeit. In der Prämessung arbeiteten die Probanden 0,08\% der Zeit im RULA Score 2, dessen prozentueller Zeitanteil sich marginal auf 0\% in der Postmessung verringerte (Abb. 44). 


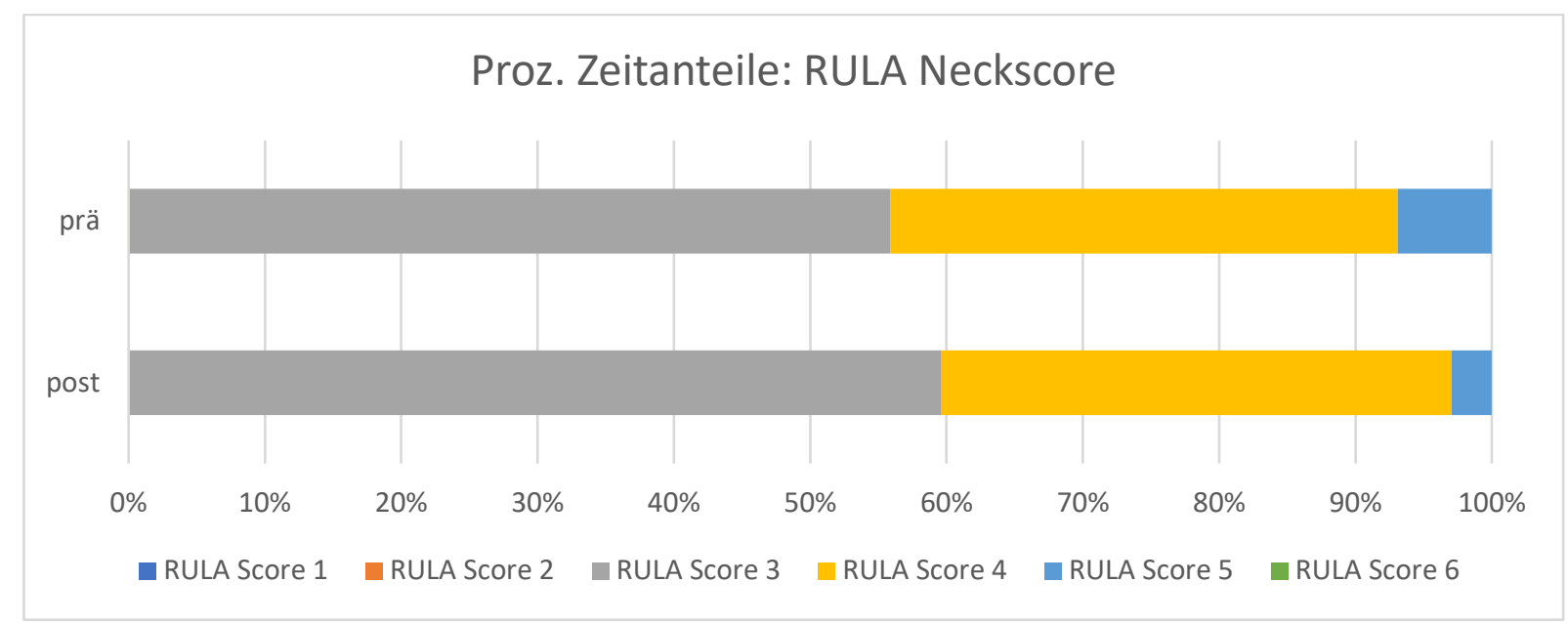

Abbildung 44: Proz. Zeitanteile: RULA Nacken (Neck)

4.2.8 Prä-Post-Auswertung der Bewertungsparameter nach relativer zeitlicher Verteilung - Oberkörper

Tabelle 14 zeigt die relative zeitliche Verteilung der Bewertungsparameter in Prozent für den Oberkörper im Prä-Post-Vergleich. Der statistische Signifikanzwert liegt bei $\mathrm{p}=0,463$. In den Bewertungsparametern RULA Score 5 und 6 lag der prozentuelle Zeitanteil für beide Messungen bei 0\%. Im RULA Score 4, der als ergonomische Risikoschwelle definiert ist, lag der Anteil vor dem Trainingszeitraum bei 2,47\% und danach bei $1,95 \%$. Die Probanden arbeiteten nach der Intervention marginal weniger im gesundheitlich riskanten Bewertungsbereich.

Tabelle 18: Prozentuelle Zeitanteile: RULA Oberkörper (Trunk)

Fett Markierung: Score wird gesundheitlich riskant eingestuft

$\begin{array}{lllllll} & \text { RULA } & \text { RULA } & \text { RULA } & \text { RULA } & \text { RULA } & \text { RULA } \\ \text { prä } & \text { Score } 1 & \text { Score } 2 & \text { Score 3 } & \text { Score 4 } & \text { Score 5 } & \text { Score 6 } \\ \text { post } & 0,51 \% & 74,15 \% & 22,88 \% & \mathbf{2 , 4 7 \%} & \mathbf{0 , 0 0 \%} & \mathbf{0 , 0 0 \%} \\ & 2,25 \% & 76,71 \% & 19,09 \% & \mathbf{1 , 9 5 \%} & \mathbf{0 , 0 0 \%} & \mathbf{0 , 0 0 \%}\end{array}$

Die Anteile des RULA Score 3 waren bei der Prämessung 22,88\% und bei der Postmessung 19,09\% und verringerten sich somit geringfügig. Zwischen den Anteilen des RULA Score 2 lassen sich nur kleine Unterschiede beschreiben zwischen prä 74,15\% und post 76,71\%. Der Anteil des RULA Scores 1 erhöhte geringfügig sich von 0,51\% vor der Trainingsintervention auf 2,25\% nach der Trainingsintervention (Abb. 45). 


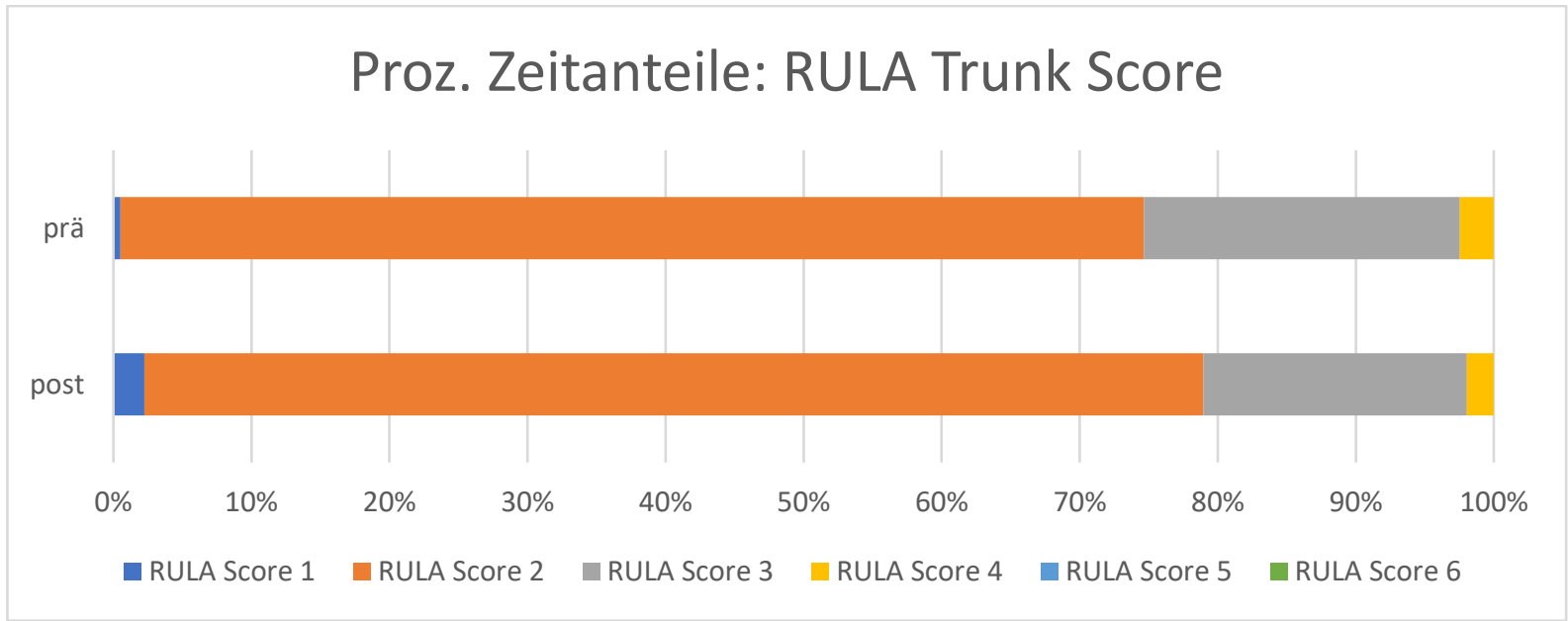

Abbildung 45: Prozentuelle Zeitanteile: RULA Oberkörper(Trunk)

4.2.9 Prä-Post-Auswertung der Bewertungsparameter nach relativer zeitlicher Verteilung - RULA Gesamtscore

Tabelle 15 zeigt die relative zeitliche Verteilung der Bewertungsparameter in Prozent für den RULA Gesamtscore im Prä-Post-Vergleich. Statistisch liegt der Signifikanzwert bei $\mathrm{p}=0,523$. Der prozentuelle Zeitanteil des als gesundheitlich riskant definierten RULA Score 7 verändert sich geringfügig von 75,88\% in der ersten Messung auf 81,30\% in der zweiten Messung. Der Zeitanteil in dem eine weiterführende Untersuchung sowie sofortige Änderung der Arbeitsweise empfohlen wird erhöht sich somit geringfügig.

Tabelle 19: Prozentuelle Zeitanteile: RULA Gesamtscore

Fett Markierung: Score wird gesundheitlich riskant eingestuft

$\begin{array}{llllllll} & \text { RULA } & \text { RULA } & \text { RULA } & \text { RULA } & \text { RULA } & \text { RULA } & \text { RULA } \\ & \text { Score 1 } & \text { Score 2 } & \text { Score 3 } & \text { Score 4 } & \text { Score 5 } & \text { Score 6 } & \text { Score 7 } \\ \text { prä } & 0,00 \% & 0,00 \% & 0,00 \% & 0,10 \% & 2,75 \% & 21,28 \% & \mathbf{7 5 , 8 8 \%} \\ \text { post } & 0,00 \% & 0,00 \% & 0,00 \% & 0,15 \% & 2,14 \% & 16,40 \% & \mathbf{8 1 , 3 0 \%}\end{array}$

Im RULA Score 6 verbrachten die Probanden während der Prämessung 21,28\% und während der Postmessung etwas weniger mit 16,4\% der Zeit. Die beschreibbaren Unterschiede im RULA Score 5 sind marginal zwischen prä 2,75\% und post 2.14\%. Insgesamt verbringen die Probanden im Vergleich während der Postmessung weniger Zeit in den RULA Scores 5 und 6, für welche der RULA Arbeitsbogen eine weitere Untersuchung und baldige Änderung der Arbeitsweise empfiehlt. Im RULA Score 4 arbeiteten die Probanden während der Prämessung in $0,1 \%$ und während der Postmessung in $0,15 \%$ der Zeit. Der Zeitanteil für den RULA Score 3 liegt für beide Messungen jeweils bei $0 \%$. Somit erhöht sich der Zeitanteil, den die Probanden in RULA Scores 3 und 4 verbracht 
haben minimal.Für diese Scores empfiehlt RULA eine weitere Untersuchung und eine eventuell notwendige Veränderung der Arbeitsweise. Die prozentuellen Anteile der RULA Scores 2 und 1 liegen jeweils für Prä- und Postmessung bei 0\%. Die Probanden arbeiteten also zu keinem Zeitpunkt in einer nach dem RULA Arbeitsbogen akzeptablen Arbeitshaltung (Abb. 46).

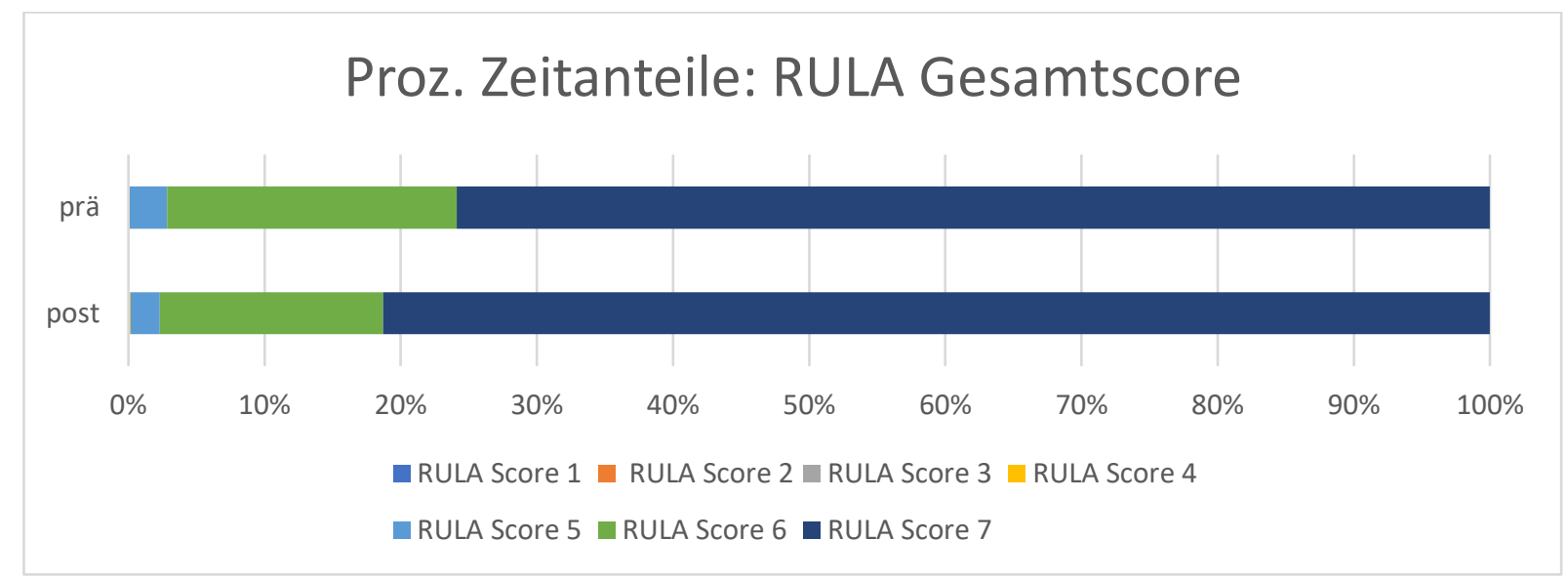

Abbildung 46: Proz. Zeitanteile: RULA Gesamtscore

\subsection{Prä-Post-Auswertung der riskanten Bewertungsparameter nach relativer zeit- licher Verteilung und Berufsgruppe}

Die als riskant definierten Bewerbungsparameter (Risky Scores) werden in diesem Kapitel anhand ihrer relativen Zeitverteilung im Prä-Post-Vergleich dargestellt. Die Risky Scores sind definiert als alle RULA Scores ab der in Kapitel 4.2 aufgeführten Risikoschwellen. Da die Probanden, außer für die Nackenregion, in jeweils nur einem riskanten Bewegungsparameter pro Körperregion gearbeitet haben sind für diese Regionen die Veränderungen der Risky Scores bereits übersichtlich im Kapitel 4.2 dargestellt. In der Nackenregion verbrachten die Probanden während der Prä- und Postmessung Zeit in mehreren Risky Scores, deren gemeinsamer Anteil an der Gesamtzeit für eine bessere Übersicht in diesem Kapitel nochmals beschrieben wird. In der statistischen Auswertung der Risky Scores aller ausgewerteten Regionen wurden nur für die Nackenregion ein signifikanter Unterschied $(\mathrm{p} \leq 0,05)$ im Prä-Post-Vergleich festgestellt. Der Signifikanzwert lag bei $\mathrm{p}=0,035$. Exemplarisch wird anhand des RULA Risky Neckscore in diesem Kapitel der unterschiedliche Einfluss der Trainingsintervention auf die gesamte Kohorte sowie die isolierte Gruppe der Z $\overparen{A}$ deskriptiv beschrieben (Abb. 47). 


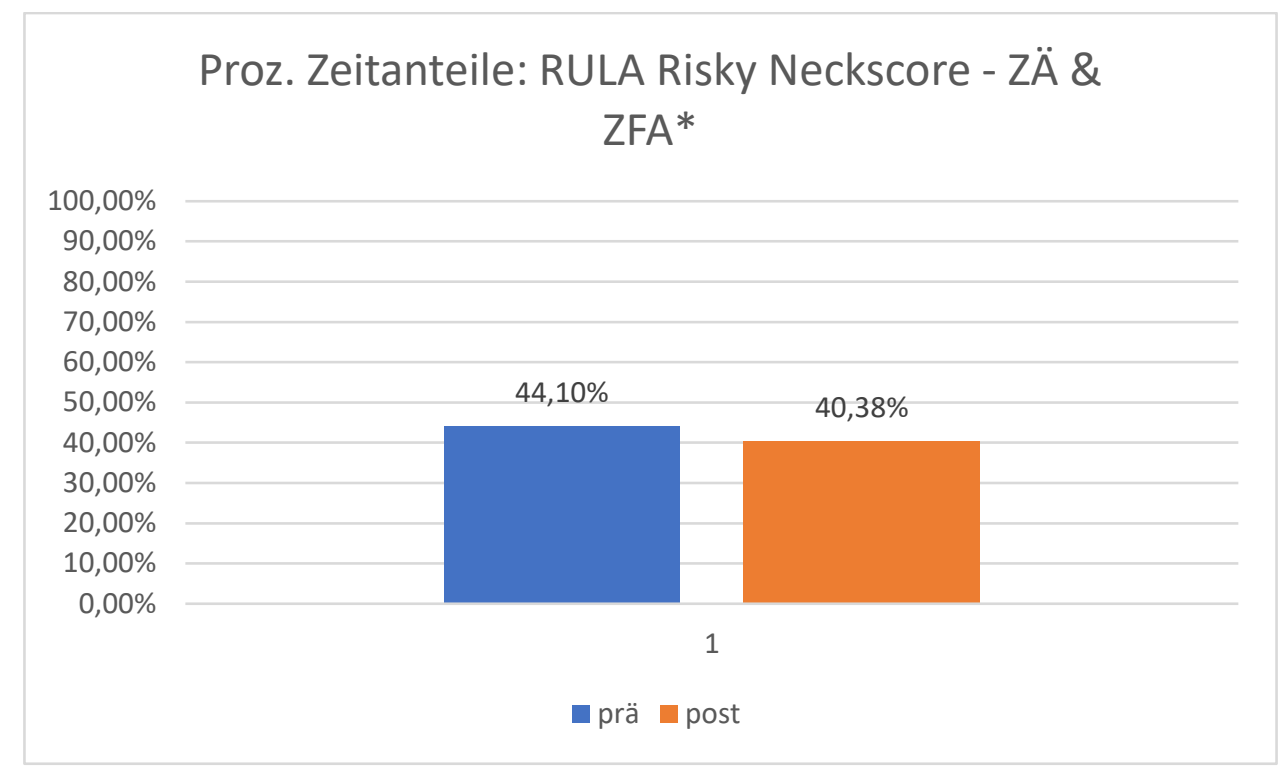

Abbildung 47: Proz. Zeitanteile RULA riskante Nackenwerte für alle Probanden (ZÄ \& ZFAs); signifikante $(p \leq 0,05)$ Unterschiede im Prä-Post-Vergleich mit * markiert

Statistisch lässt sich zwischen der Gesamtkohorte und den ZÄ kein statistisch signifikanter Unterschied ( $\mathrm{p} \geq 0,05)$ bezüglich des prozentuellen Zeitanteils der Risky Scores beschreiben. Für die nach ZÄ aufgeteilte Probandengruppe lässt sich deskriptiv in der Prämessung ein marginaler Unterschied für den prozentuellen Zeitanteil, der in riskanten Bewegungsparametern gearbeitet wurde, erkennen. Die ZÄ verbrachten 43,85\% der Zeit in einem Risky Neckscore und somit 0,88\% marginal weniger als die gesamte Kohorte (Abb.48) mit 44,1\%. Während der Postmessung arbeiteten die Z̈̈ 39,72\% der Zeit in einer riskanten Arbeitsweise und damit 0,66\% weniger als die das gesamte Probandenkollektiv.

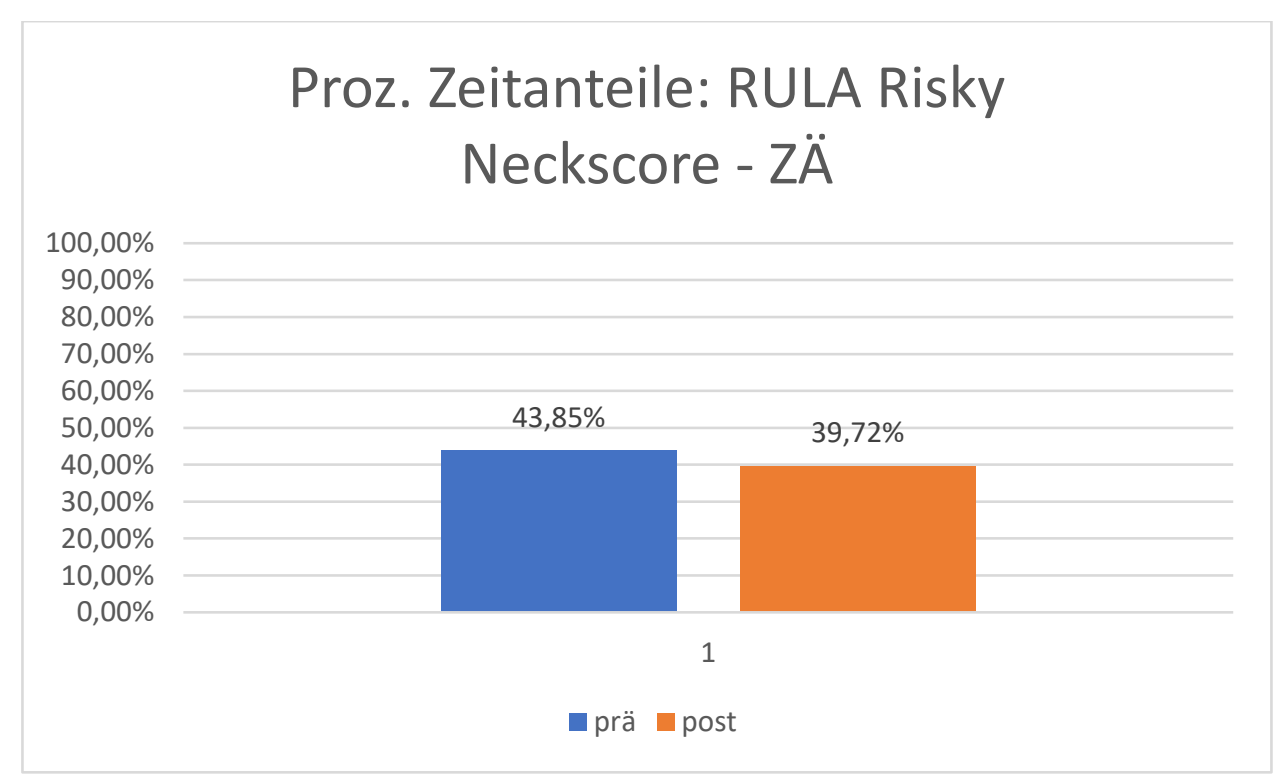

Abbildung 48: Proz. Zeitanteile RULA riskante Nackenwerte für Zahnärzte 
In der Einzelbetrachtung lässt sich bei den Zahnärzten beschreiben, dass die Zeit, welche in Risky Scores verbracht wird, sich von Prä- zu Postmessung mit 4,13\% geringfügig verringert hat. Für das gesamte Probandenkollektiv ist der Wert der relativen Zeitanteile, die in riskanten Bewegungsparametern verbracht wurden, in der Nackenregion vor der Trainingsintervention bei 44,1\%. Nach der Trainingsintervention arbeiteten die Probanden 40,38\% der gemessenen Zeit in einem Risky Score. Der Wert verringerte sich von Prä- zu Postmessung um 3,72\%. Somit verändern sich der relative Zeitanteil, die beide Gruppen in Risky Scores verbrachten, in ähnlichen Ausmaßen.

\subsection{Prä-Post-Auswertung der Gelenkwinkel und Gelenkpositionen nach relativer zeitlicher Verteilung}

Für die Auswertung mit RULA wurden in den biomechanischen Messungen die Gelenkwinkel erfasst. Die relative Häufigkeit im Verhältnis zur Gesamtzeit, mit der ein Gelenkwinkel gemessen wurde, wird im folgenden Kapitel für die bewerteten Körperregionen im Prä-Post-Vergleich beschrieben. Einige RULA Scores setzen sich aus verschiedenen Bewegungsmustern in der jeweiligen Region zusammen, die entsprechenden Gelenkwinkel dieser Bewegungen werden im folgenden Kapitel separat beschrieben. Die Bewertung der Gelenkwinkel nach dem RULA Bogen ist von einem niedrigen zu einem hohen Score anhand von hell nach dunkel abgestuften Grautönen in den unten abgebildeten Histogrammen hinterlegt. Die dickgezeichnete Linie beschreibt den Medianwert des jeweiligen Parameters und wird im Folgenden als Medianlinie bezeichnet. Die dünngezeichneten Linien sind die Linien des ersten bzw. dritten Quartils. Alle Gelenkwinkel wurden im Prä-Post-Vergleich statistisch gegeneinander getestet. Aus Gründen der Übersichtlichkeit werden im Folgenden die Signifikanzwerte nur bei einem statistisch signifikanten Unterschied $(p \leq 0,05)$ angegeben.

4.4.1 Prä-Post-Auswertung der Gelenkwinkel nach relativer zeitlicher Verteilung - Oberarm linksseitig

Für die Oberarmregion wurden drei unterschiedliche Bewegungsmuster und dazugehörige Gelenkwinkel betrachtet. Statistisch kann keine signifikante Veränderung $(p \geq 0,05)$ 
der Gelenkwinkel zwischen Prä- und Postmessung beschrieben werden. Im linken Oberarm lässt sich im Vergleich der Prä- und Postmessung deskriptiv nur eine geringfügige Varianz zwischen der relativen Häufigkeit der angewählten Gelenkwinkel ausmachen. Den größten relativen Zeitanteil verbringen die Probanden jeweils im Bereich zwischen 0 und $30^{\circ}$. Mit der relativ größten Häufigkeit wurde ein Gelenkwinkel um $15^{\circ}$ gemessen. Die in Abb 49 dargestellte blaue Medianlinie der Postmessung verschiebt sich im Bereich von $0^{\circ}$ bis $35^{\circ}$ marginal in Richtung größerer positiver Gelenkwinkel und somit in Richtung Flexion.

Abb 50 zeigt die Elevation der linken Schulter. Statistisch lassen sich keine signifikanten Veränderungen ( $p \geq 0,05)$ beschreiben. Die Medianlinien der Prä- und Postmessung variieren auch deskriptiv nur marginal. In beiden Messungen verbringen die Probanden den relativ größten Anteil zwischen $-13^{\circ}$ und $-3^{\circ}$. Somit arbeiten sie zum deutlichen Großteil nicht mit elevierter linker Schulter. Im Bereich um $-8^{\circ}$ wurde der Gelenkwinkel mit der relativ größten Häufigkeit eingenommen. Er wurde minimal öfter während der Postmessung verzeichnet.

In Abb 51 ist die Abduktion des linken Oberarms dargestellt. Statistisch lassen sich keine signifikanten Unterschiede $(\mathrm{p} \geq 0,05)$ zwischen den Messungen vor und nach der Intervention beschreiben. Es zeigen sich deskriptiv zwischen den Linien für Prä- und Postmessung nur minimale Unterschiede. Die Probanden arbeiteten den größten Zeitanteil in einem Gelenkwinkel zwischen $10^{\circ}$ und $35^{\circ}$ und somit noch nicht in einer abduzierten Haltung des Oberarms. Am öftesten wurde ein Gelenkwinkel von $20^{\circ}$ gemessen. In der Postmessung wurde geringfügig öfter in einem Gelenkwinkel zwischen $15^{\circ}$ und $20^{\circ}$ gearbeitet. 

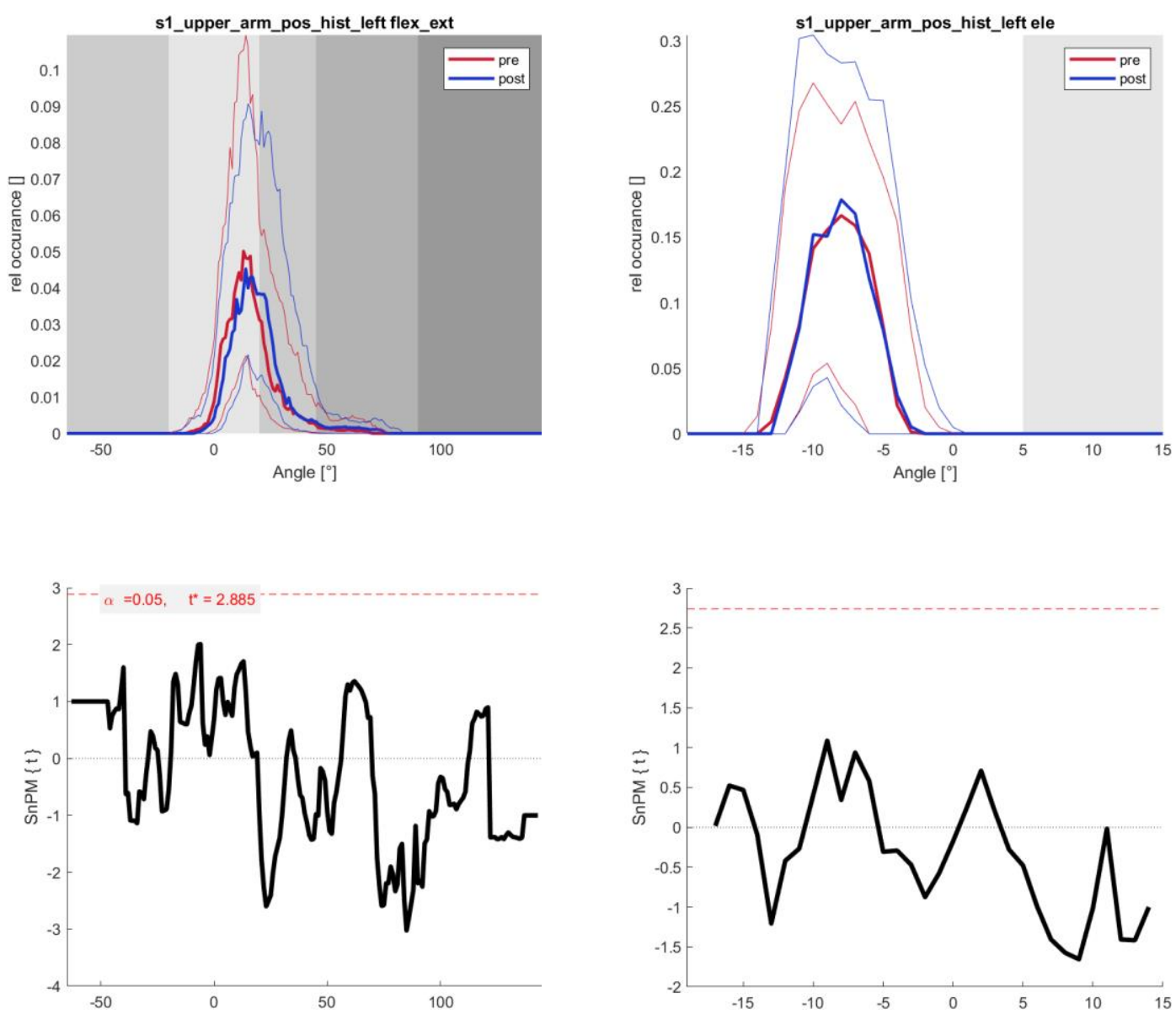

Abbildung 49: Gelenkwinkel Oberarm linksseitig - Flexion/Extension

Oben: Abszisse = Gelenkwinkel (positive Gradzahlen = Flexion, negative Gradzahlen $=$ Extesion $) ;$ Ordinate $=$ rel. zeitl. Verteilung (Gesamtzeit = 1); Graustufen = RULA Scores aufsteigend von hell (niedriger Score) nach dunkel (hoher Score) hinterlegt

Unten: Abzisse = Gelenkwinkel; Ordinate

$=t$-Werte, rote Linie $=$ Signifikanzgrenze 

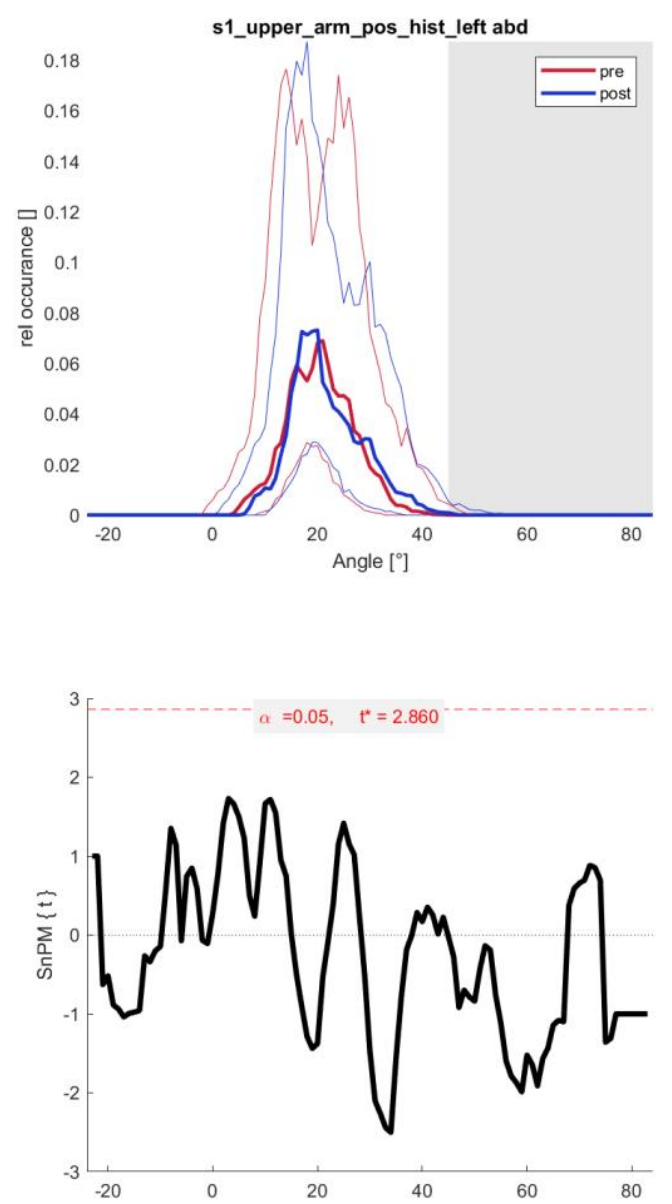

Abbildung 51: Gelenkwinkel Oberarm linksseitig - Abduktion

Oben: Abszisse $=$ Gelenkwinkel $\left(\right.$ Abduktion $\left.>45^{\circ}\right)$; Ordinate $=$ rel. zeitl. Verteilung $($ Gesamtzeit $=1)$; Graustufen = RULA Scores aufsteigend von hell (niedriger Score) nach dunkel (hoher Score) hinterlegt Unten: Abzisse $=$ Gelenkwinkel; Ordinate $=t$-Werte, rote Linie $=$ Signifikanzgrenze

4.4.2 Prä-Post-Auswertung der Gelenkwinkel/-position nach relativer zeitlicher Verteilung - Unterarm linksseitig

Für die Unterarmregion wurden zwei unterschiedliche Bewegungsmuster und dazugehörige Gelenkwinkel betrachtet. Es kann statistisch kein signifikanter Unterschied $(p \geq 0,05)$ zwischen Prä- und Postmessung beschrieben werden. Abb. 52 zeigt im Prä-Postvergleich die Flexion und Extension des linken Unterarms. Zwischen den Medianlinien der Präund Postmessung lassen sich nur minimalste Varianzen ausmachen. Während beiden Messungen arbeiteten die Probanden größtenteils in einem Gelenkwinkel zwischen $80^{\circ}$ und $120^{\circ}$. Am meisten arbeiteten die Probanden in einem Gelenkwinkel um die $100^{\circ}$. In Abb. 53 ist die Gelenkposition des linken Unterarms dargestellt. Es lässt sich statistisch keine signifikante Veränderung $(\mathrm{p} \geq 0,05)$ der Gelenkwinkel zwischen den Messungen 
beschreiben. Zwischen der Prä- und Postmessung kann nur sehr vereinzelt ein geringer Unterschied deskriptiv beschrieben werden. In beiden Messungen arbeiteten die Probanden den zeitlich größten Anteil mit dem linken Unterarm in einer Position zwischen $0,2 \mathrm{~m}$ und $-0,35 \mathrm{~m}$ und somit über die Körpermitte hinaus. Am öftesten behandelten sie in einer Gelenkposition von $-0,25 \mathrm{~m}$.
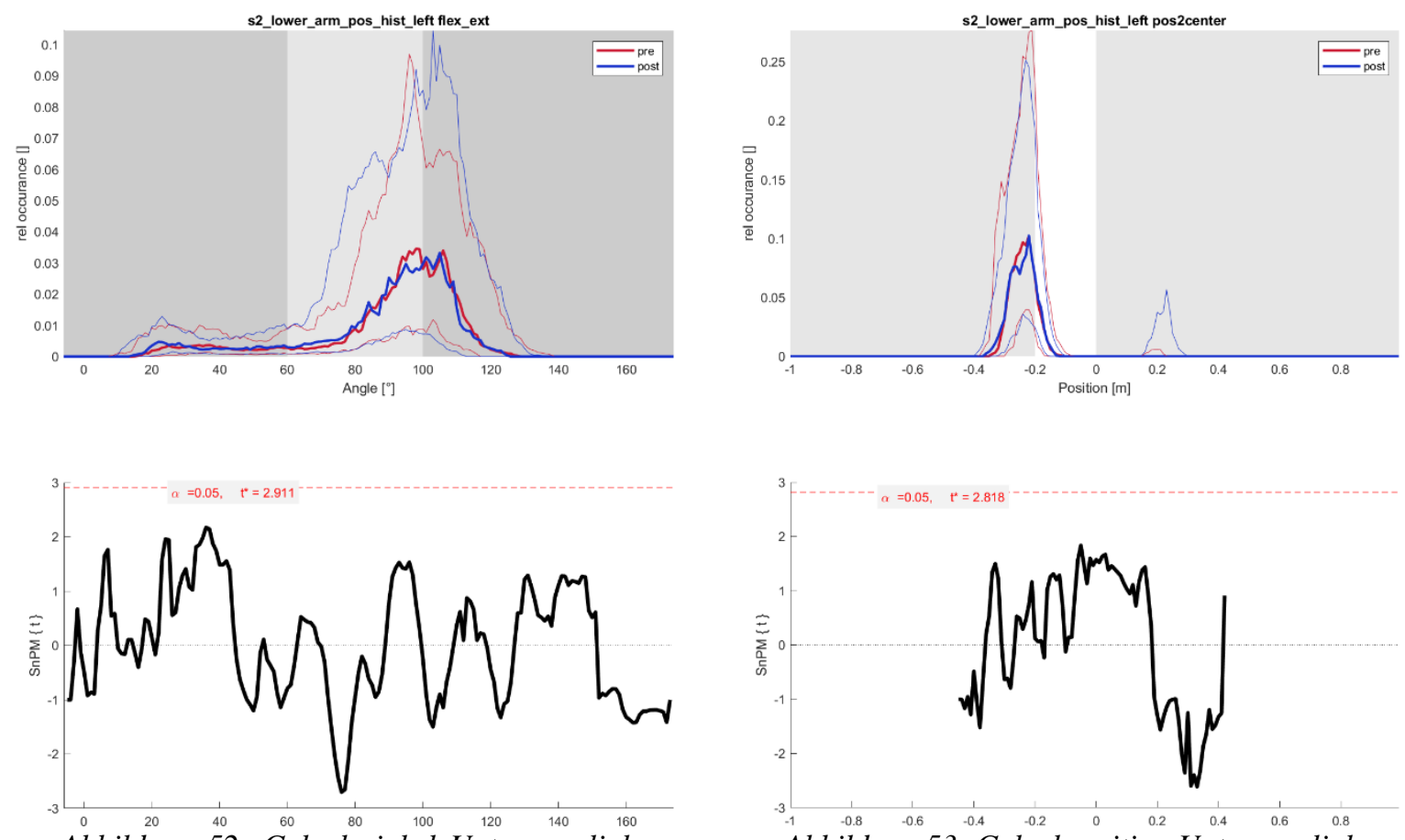

Abbildung 52: Gelenkwinkel Unterarm linksseitig - Flexion/Extension

Oben: Abszisse = Gelenkwinkel (positive Gradzahlen = Flexion); Ordinate = rel. zeitl . Verteilung (Gesamtzeit $=1)$; Graustufen = RULA Scores aufsteigend von hell (niedriger Score) nach dunkel (hoher Score) hinterlegt Unten: Abzisse = Gelenkwinkel; Ordinate $=t$ Werte, rote Linie $=$ Signifikanzgrenze

Abbildung 53: Gelenkposition Unterarm linksseitig - Position Transversalebene Oben: Abszisse $=$ Position (Arbeitet Unterarm außerhalb Körpermitte; über Körpermitte hinaus); Ordinate = rel. zeitl. Verteilung (Gesamtzeit $=1)$; Graustufen $=$ RULA Scores aufsteigend von hell (niedriger Score) nach dunkel (hoher Score) hinterlegt

Unten: Abzisse = Gelenkwinkel; Ordinate $=t$ Werte, rote Linie $=$ Signifikanzgrenze

4.4.3 Prä-Post-Auswertung der Gelenkwinkel nach relativer zeitlicher Verteilung - Handgelenk \& Rotation linksseitig

Für Handgelenk und Rotation wurden zwei unterschiedliche Bewegungsmuster und dazugehörige Gelenkwinkel betrachtet. Die statistische Auswertung konnte keine signifikante Veränderung $(\mathrm{p} \geq 0,05)$ für den Gelenkwinkel der Flexion und Extension des linken Handgelenks feststellen. In der Auswertung der Gelenkwinkel des Handgelenks gibt es bei den Bewegungen der Flexion und Extension zwischen Prä- und Postmessung nur geringfügige Unterschiede. Sowohl vor als auch nach der Trainingsintervention arbeiten die 
Probanden zum Großteil in einem Wertbereich zwischen $-55^{\circ}$ und $-20^{\circ}$ und folglich einer Flexion des Handgelenks. Der am meisten gemessene Gelenkwinkel lag bei $-35^{\circ}$. Die in Abb. 54 dargestellte blaue Mediankurve der Postmessung ist marginal in Richtung einer stärkeren Flexion verschoben.

Abb. 55 zeigt die Deviation des linken Handgelenks unterschieden in Radial- und Ulnardeviation. Statistisch wurde ein Gelenkwinkel im Bereich um $0^{\circ}$ während der Prämessung signifikant $(p \leq 0,01)$ öfter eingenommen im Vergleich zur Postmessung. Der Signifikanzwert lag hier bei $p=0,003$. Dieser Unterschied lässt sich auch deskriptiv beschreiben. Es lässt sich außerdem im deskriptiven Vergleich der Medianlinien von Prä- und Postmessung erkennen, dass im Bereich um $15^{\circ}$ während der Postmessung im relativen Zeitvergleich öfter zur Einnahme dieses Gelenkwinkels kam. Während der Prämessung arbeiteten die Probanden vorwiegend im Bereich zwischen $-10^{\circ}$ und $25^{\circ}$; am öftesten bei $11^{\circ}$. Während der Postmessung arbeiteten sie vermehrt im Bereich zwischen $-5^{\circ}$ und $+30^{\circ}$; am öftesten bei $15^{\circ}$. Die Mediankurve der Postmessung verschiebt sich im Vergleich in Richtung einer verstärkten Radialdeviation. 

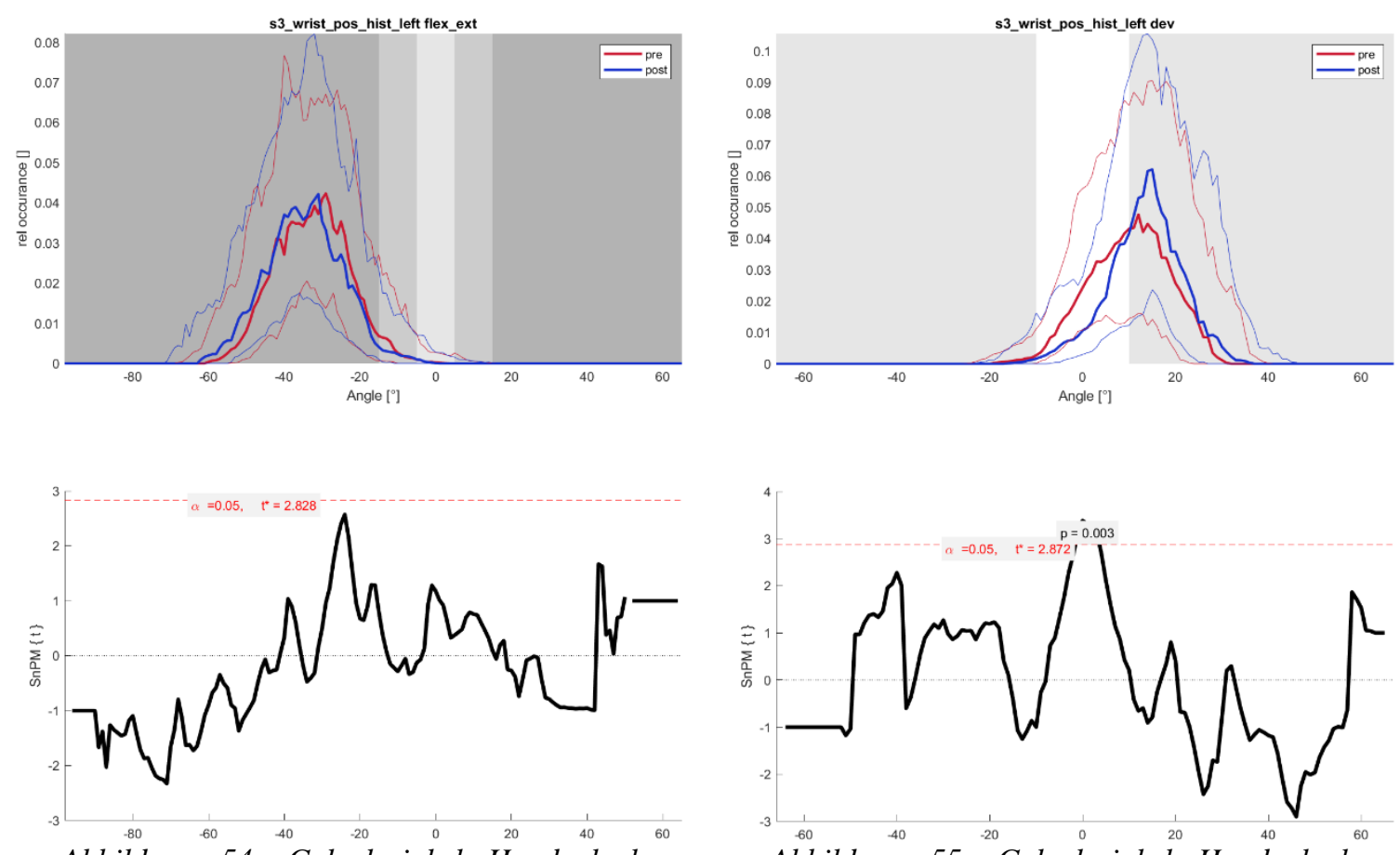

Abbildung 54: Gelenkwinkel Handgelenk Abbildung 55: Gelenkwinkel Handgelenk linksseitig - Deviation

Oben: Abszisse = Gelenkwinkel (positive Gradzahlen = Radialdeviation, negative Gradzahlen = Ulnardeviation $) ;$ Ordinate $=$ rel. zeitl. Verteilung (Gesamtzeit = 1); Graustufen = RULA Scores aufsteigend von hell (niedriger Score) nach dunkel (hoher Score) hinterlegt

Unten: Abzisse = Gelenkwinkel; Ordinate = $t$-Werte, rote Linie $=$ Signifikanzgrenze

4.4.4 Prä-Post-Auswertung der Gelenkwinkel nach relativer zeitlicher Verteilung - Oberarm rechtsseitig

Abb. 56 veranschaulicht die Flexion/Extension des rechten Oberarms. Statistisch ließen sich keine signifikanten Unterschiede $(\mathrm{p} \geq 0,05)$ zwischen den Messungen vor und nach der Trainingsintervention beschreiben. Im Prä-Post-Vergleich konnten deskriptiv nur geringfügige Unterschiede zwischen der relativen Häufigkeit der gemessenen Gelenkwinkel beschrieben werden. Die Probanden arbeiteten in beiden Messungen größtenteils in einem Wertebereich von $0^{\circ}$ bis $60^{\circ}$ mit jeweils der höchsten relativen Häufigkeit um $25^{\circ}$. Die Probanden arbeiteten somit größtenteils in einer Nackenflexion. Im direkten Vergleich mit der Prämessung zeigt die Postmessung eine marginal höhere relative Häufigkeit um den Gelenkwinkel von $25^{\circ}$. 
In Abb. 57 ist die Elevation des rechten Oberarms dargestellt. Statistisch konnte kein signifikanter $((\mathrm{p} \geq 0,05)$ Unterschied zwischen den beiden Messungen beschrieben werden. Im deskriptiven Vergleich der relativen Häufigkeiten für Prä- und Postmessung ließen sich nur geringgradige Unterschiede ausmachen. Größtenteils arbeiteten die Probanden in einem Wertebereich zwischen $<-10^{\circ}$ und $>+5^{\circ}$. Am meisten arbeiteten sie in einem Gelenkwinkel um $-5^{\circ}$ und somit nicht mit einer Elevation der rechten Schulter. Während der Postmessung arbeiteten die Probanden marginal mehr in einem Gelenkwinkelbereich von -5 bis $-10^{\circ}$.

Bei Betrachtung der Abduktion des rechten Oberarms, ließen sich statistisch keine signifikanten Unterschiede ( $\mathrm{p} \geq 0,05)$ zwischen Prä- und Postmessung feststellen. Deskriptiv war nur eine geringe Varianz zwischen den Medianlinien der relativen Häufigkeiten für Prä- und Postmessung erkennbar (Abb. 58). Die Probanden arbeiteten zum Großteil in Gelenkwinkeln zwischen $10^{\circ}$ und $50^{\circ}$. Die höchste relative Häufigkeit lässt sich für beide Messungen jeweils im Bereich um $15^{\circ}$ beschreiben, wobei in der Postmessung geringfügig öfter ein Gelenkwinkel im Bereich zwischen $15^{\circ}$ und $20^{\circ}$ gemessen wurde. Während beiden Messungen arbeiteten die Probanden zu einem deutlichen Mehranteil im Bereich $<45^{\circ}$ und somit nicht mit abduziertem Oberarm. Die relative Häufigkeit für Gelenkwin$\mathrm{kel} \leq 45^{\circ}$ war in beiden Messungen gering. 

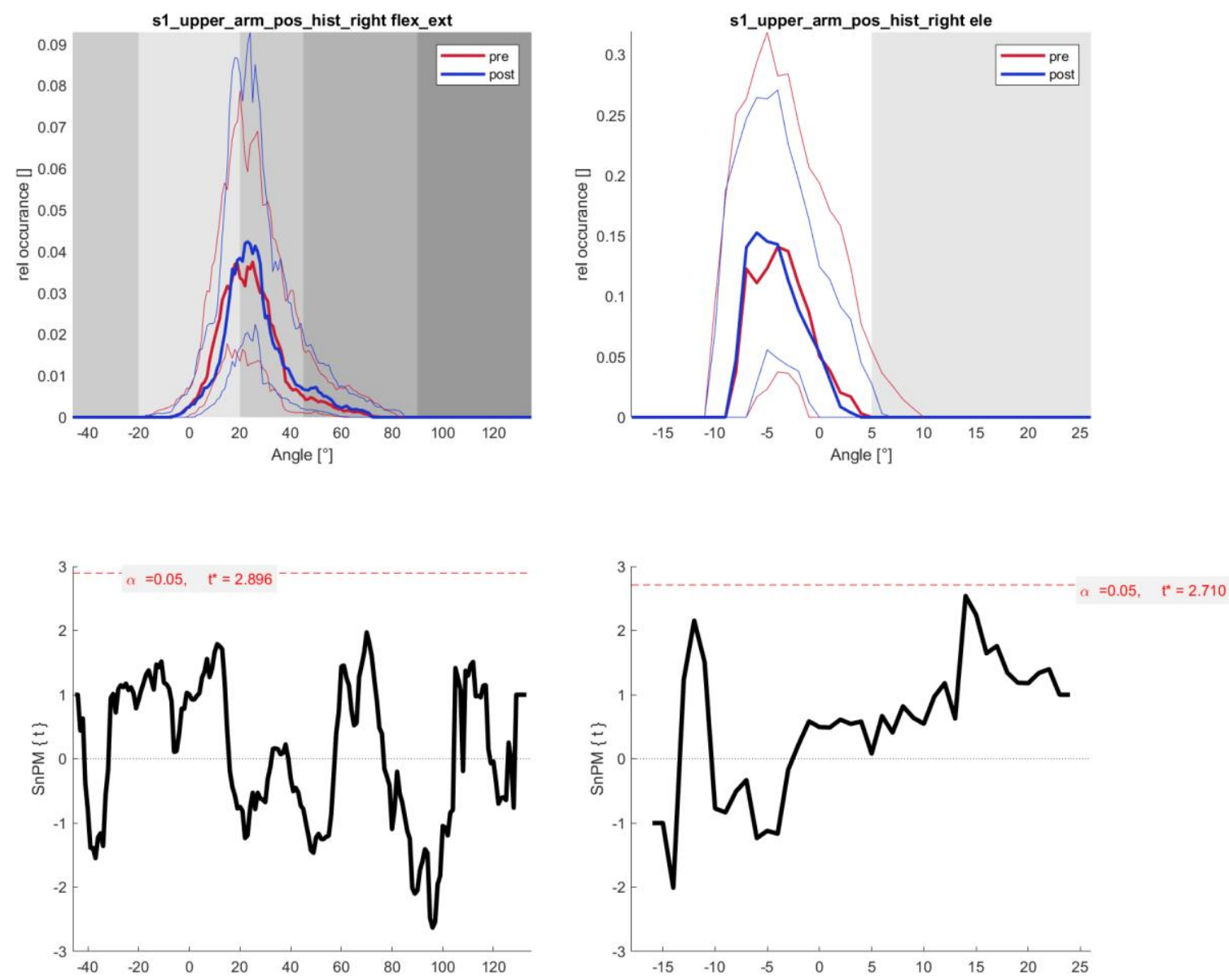

Abbildung 56: Gelenkwinkel Oberarm rechtsseitig - Flexion/Extension

Oben: Abszisse = Gelenkwinkel (positive Gradzahlen = Flexion, negative Gradzahlen = Extesion $) ;$ Ordinate $=$ rel. zeitl. Verteilung (Gesamtzeit $=1)$; Graustufen $=$ RULA Scores aufsteigend von hell (niedriger Score) nach dunkel (hoher Score) hinterlegt

Unten: Abzisse = Gelenkwinkel; Ordinate $=t$-Werte, rote Linie $=$ Signifikanzgrenze

Abbildung 57: Gelenkwinkel Oberarm rechtsseitig - Elevation Schulter

Oben: Abszisse $=$ Gelenkwinkel $\left(\right.$ Elevation $\left.>5^{\circ}\right)$; Ordinate $=$ rel. zeitl. Verteilung $($ Gesamtzeit $=1)$; Graustufen $=$ RULA Scores aufsteigend von hell (niedriger Score) nach dunkel (hoher Score) hinterlegt

Unten: Abzisse $=$ Gelenkwinkel; Ordinate $=t$ Werte, rote Linie $=$ Signifikanzgrenze 

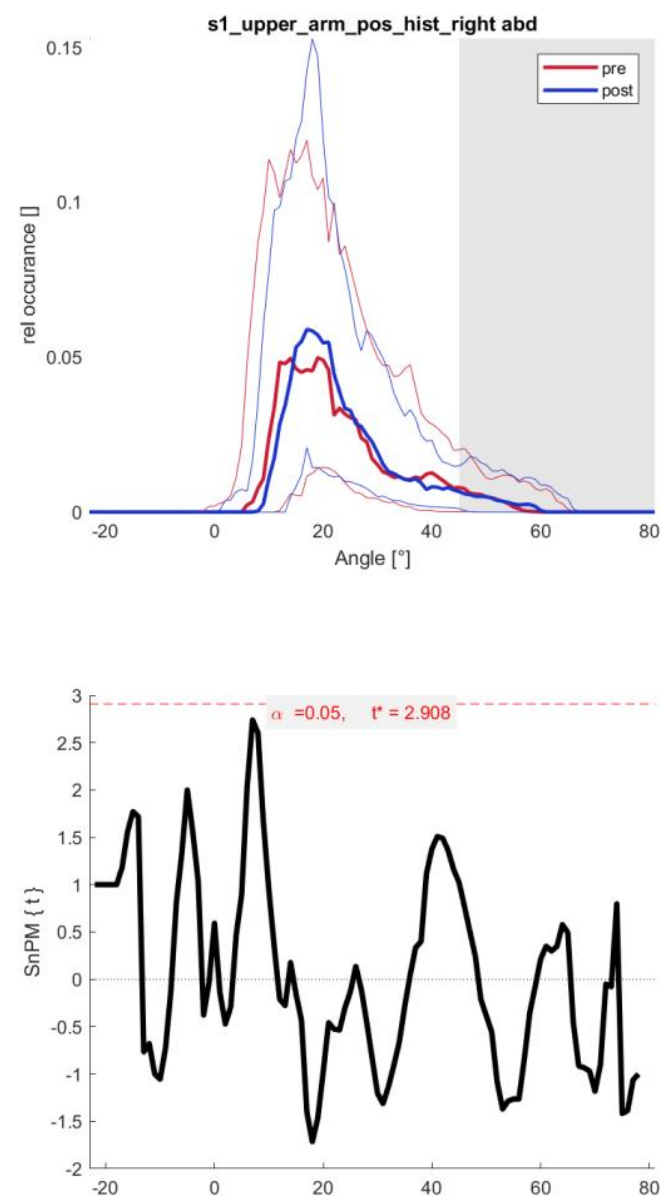

Abbildung 58: Gelenkwinkel Oberarm rechtsseitig - Abduktion

Oben: Abszisse = Gelenkwinkel $($ Abduktion > 45); Ordinate $=$ rel . zeitl. Verteilung $($ Gesamtzeit $=$ 1); Graustufen = RULA Scores aufsteigend von hell (niedriger Score) nach dunkel (hoher Score) hinterlegt

Unten: Abzisse $=$ Gelenkwinkel; Ordinate $=t$-Werte, rote Linie $=$ Signifikanzgrenze

4.4.5 Prä-Post-Auswertung der Gelenkwinkel nach relativer zeitlicher Verteilung - Unterarm rechtsseitig

Abb. 59 stellt die relative Häufigkeit der Gelenkwinkel des rechten Unterarms für Flexion und Extension dar. Statistisch wurde eine Gelenkwinkel um $70^{\circ}$ in der Prämessung signifikant $(p \leq 0,01)$ öfter gemessen. Die stat. Signifikanz lag hier bei $p=0,001$. Die Probanden arbeiteten in beiden Messungen zum Großteil im Bereich zwischen $50^{\circ}$ und $110^{\circ}$. Deskriptiv lässt sich zwischen den Medianlinien der Prä- und Postmessung eine Varianz zwischen den am häufigsten gemessenen Gelenkwinkeln beschreiben. Für die Prämessung liegt der relativ am häufigsten eingenommene Gelenkwinkel im Bereich um $70^{\circ}$. Während der Postmessung lag er im Bereich um $90^{\circ}$ und somit verschoben hin zu einer stärkeren Flexion. 
In Abb. 60 ist die Gelenkposition des rechten Unterarms dargestellt. Es konnte sich statistisch keine signifikante Veränderung $(\mathrm{p} \geq 0,05)$ der Gelenkwinkel zwischen den Messungen beschreiben lassen. Zwischen der Prä- und Postmessung konnte deskriptiv vereinzelt ein geringer Unterschied beschrieben werden. In beiden Messungen arbeiteten die Probanden den zeitlich größten Anteil mit dem rechten Unterarm in einer Position zwischen 0,2m und 0,4m und somit über die Körpermitte hinaus. Während der Postmessung verbrachten die Probanden geringfügig mehr Zeit in einer Position um 0,2m und somit weniger über die Körpermitte hinaus.
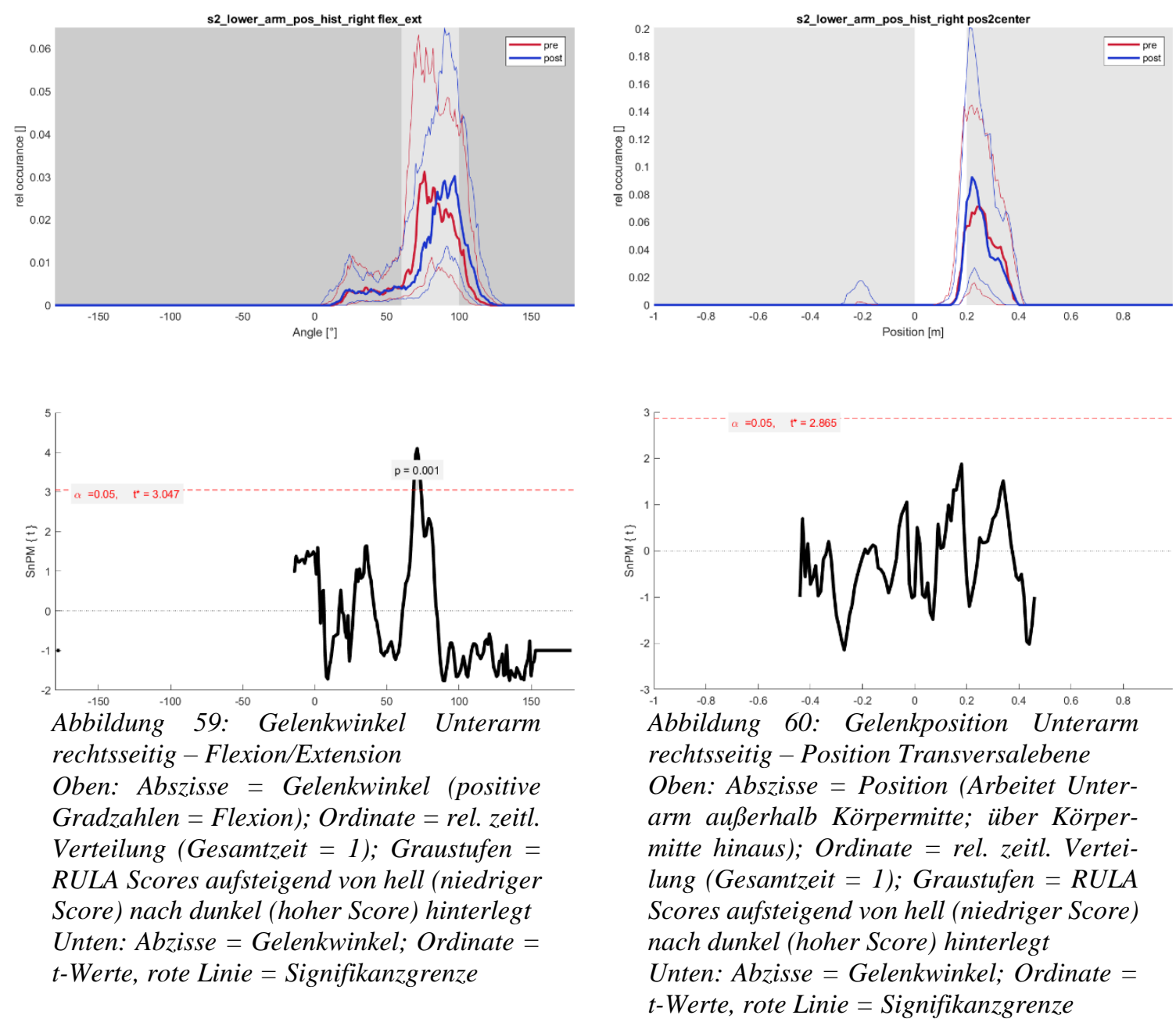
4.4.6 Prä-Post-Auswertung der Gelenkwinkel nach relativer zeitlicher Verteilung - Handgelenk \& Rotation rechtsseitig

In der Auswertung der Gelenkwinkel des Handgelenks gab es bei den Bewegungen der Flexion und Extension zwischen Prä- und Postmessung geringe Varianzen (Abb. 61). Im statistischen Vergleich ließen sich keine signifikanten Unterschiede $(p \geq 0,05)$ zwischen den beiden Messungen beschreiben. Während beiden Messungen wurde von den Probanden größtenteils Gelenkwinkel zwischen $-60^{\circ}$ und $0^{\circ}$ eingenommen. Am öftesten wurde ein Gelenkwinkel um $-45^{\circ}$ gemessen. Somit arbeiteten sie jeweils zum Großteil mit stark extendiertem rechtem Handgelenk. Im deskriptiven Vergleich ist die Mediankurve der Postmessung um $<-5^{\circ}$ in Richtung einer stärkeren Extension des Handgelenks verschoben.

Wird die Deviation des rechten Handgelenks betrachtet wurde im statistisch Prä-PostVergleich während der Prämessung signifikant $(\mathrm{p} \leq 0,01)$ öfter ein Gelenkwinkel um $-10^{\circ}$ eingenommen. Der Signifikanzwert lag hier bei $\mathrm{p}=0,001$. Deskriptiv lassen sich ebenfalls deutliche Unterschiede erkennen (Abb. 62). Während der Prämessung behandelten die Probanden größtenteils in einem Gelenkwinkelbereich zwischen $-15^{\circ}$ und $20^{\circ}$, am häufigsten wurde dabei ein Gelenkwinkel zwischen $0^{\circ}$ und $5^{\circ}$ gemessen. In der Postmessung arbeiteten die Probanden zum Großteil in einem Gelenkwinkelbereich zwischen $-15^{\circ}$ und $25^{\circ}$, am häufigsten wurde dabei ein Gelenkwinkel um $10^{\circ}$ gemessen. In beiden Messungen arbeiten die Probanden mit dem rechten Handgelenk einen deutlichen Zeitanteil in einer Radialflexion und einen geringeren Anteil in einer Ulnardeviation. Die Mediankurve für die Postmessung verschiebt sich im Vergleich zur Prämessung in Richtung einer Radialdeviation. 

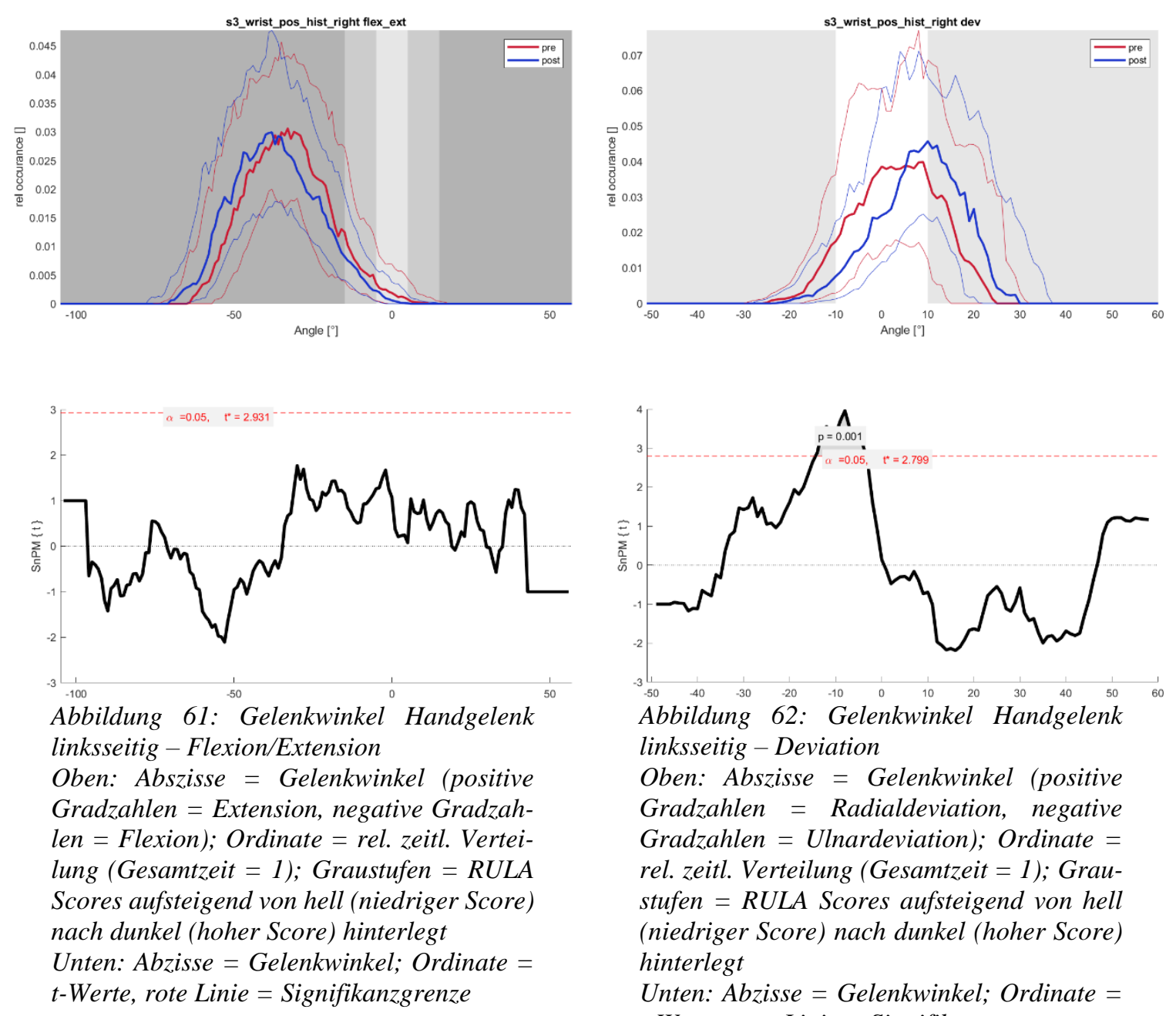

Abbildung 62: Gelenkwinkel Handgelenk linksseitig - Deviation

Oben: Abszisse = Gelenkwinkel (positive Gradzahlen = Radialdeviation, negative Gradzahlen $=$ Ulnardeviation $) ;$ Ordinate $=$ rel. zeitl. Verteilung (Gesamtzeit = 1); Graustufen = RULA Scores aufsteigend von hell (niedriger Score) nach dunkel (hoher Score) hinterlegt

Unten: Abzisse = Gelenkwinkel; Ordinate = $t$-Werte, rote Linie $=$ Signifikanzgrenze

4.4.7 Prä-Post-Auswertung der Gelenkwinkel nach relativer zeitlicher Verteilung - Nacken

Für die Nackenregion wurden drei unterschiedliche Bewegungsmuster und dazugehörige Gelenkwinkel betrachtet. Abb. 63 zeigt die Flexion bzw. Extension des Nackens. Im Gelenkwinkelbereich um $45^{\circ}$ war ein statistischer Unterschied zwischen Prä- und Postmessung beschreibbar dieser wurde während der Prämessung signifikanter $(\mathrm{p} \leq 0,01)$ öfter eingenommen. Die stat. Signifikanz lag hier bei $p=0,001$. Es lassen sich auch deskriptiv deutliche Unterschiede zwischen der relativen Häufigkeit der gemessenen Gelenkwinkel erkennen. Die Probanden arbeiteten während der Prämessung hauptsächlich in einem Gelenkwinkelbereich zwischen $20^{\circ}$ und $53^{\circ}$, die relativ größte Häufigkeit wurde im Bereich um $45^{\circ}$ gemessen. Während der Postmessung arbeiteten die Probanden hauptsächlich im Bereich von $20^{\circ}$ bis $58^{\circ}$ mit einer relativ größten Häufigkeit bei $43^{\circ}$. Die gemessenen 
Gelenkwinkel der Postmessung verschieben sich in Richtung einer stärkeren Flexion. Deskriptiv wurde ein Gelenkwinkel im Bereich von $45^{\circ}$ während der Prämessung circa 1,5 Mal so oft gemessen wie in der Postmessung.

Wird die Rotation des Nackens im Vergleich der Messungen vor und nach dem Interventionszeitraum betrachtet (Abb. 64), konnten sich statistisch keine signifikanten ( $p \geq 0,05)$ Differenzen zwischen Prä- und Postmessung beschrieben werden. Es ließen sich deskriptiv nur marginale Unterschiede beschreiben. Die Probanden arbeiteten sowohl vor als auch nach der Trainingsintervention hauptsächlich im Gelenkwinkelbereich zwischen $20^{\circ}$ und $+10^{\circ}$, mit der höchsten relativen Häufigkeit im Bereich um ca. $0^{\circ}$. Somit arbeiten die Probanden größtenteils in einer neutralen Nackenrotation, der Kopf wird im Seitenvergleich bei beiden Messungen vermehrt nach links rotiert.

Für die Beugung des Nackens in der Frontalebene konnte statistisch ein signifikanter Unterschied ( $\mathrm{p} \leq 0,05)$ zwischen Prä- und Postmessung im Bereich um $+15^{\circ}$ beschrieben werden. Der Signifikanzwert lag hier bei $\mathrm{p}=0,030$. Im Vergleich der beiden Messungen waren deskriptiv nur geringe Varianzen beschreibbar (Abb. 65). Hauptsächlich arbeiten die Probanden in beiden Messungen im Bereich der Gelenkwinkel von -15 bis +15 , dabei wurde die größte relative Häufigkeit im Bereich um einen Gelenkwinkel von $5^{\circ}$ gemessen. Die Mediankurve der Postmessung ist dabei geringfügig in Richtung negativer Gradzahlen und somit einer Linksbeugung verschoben. 

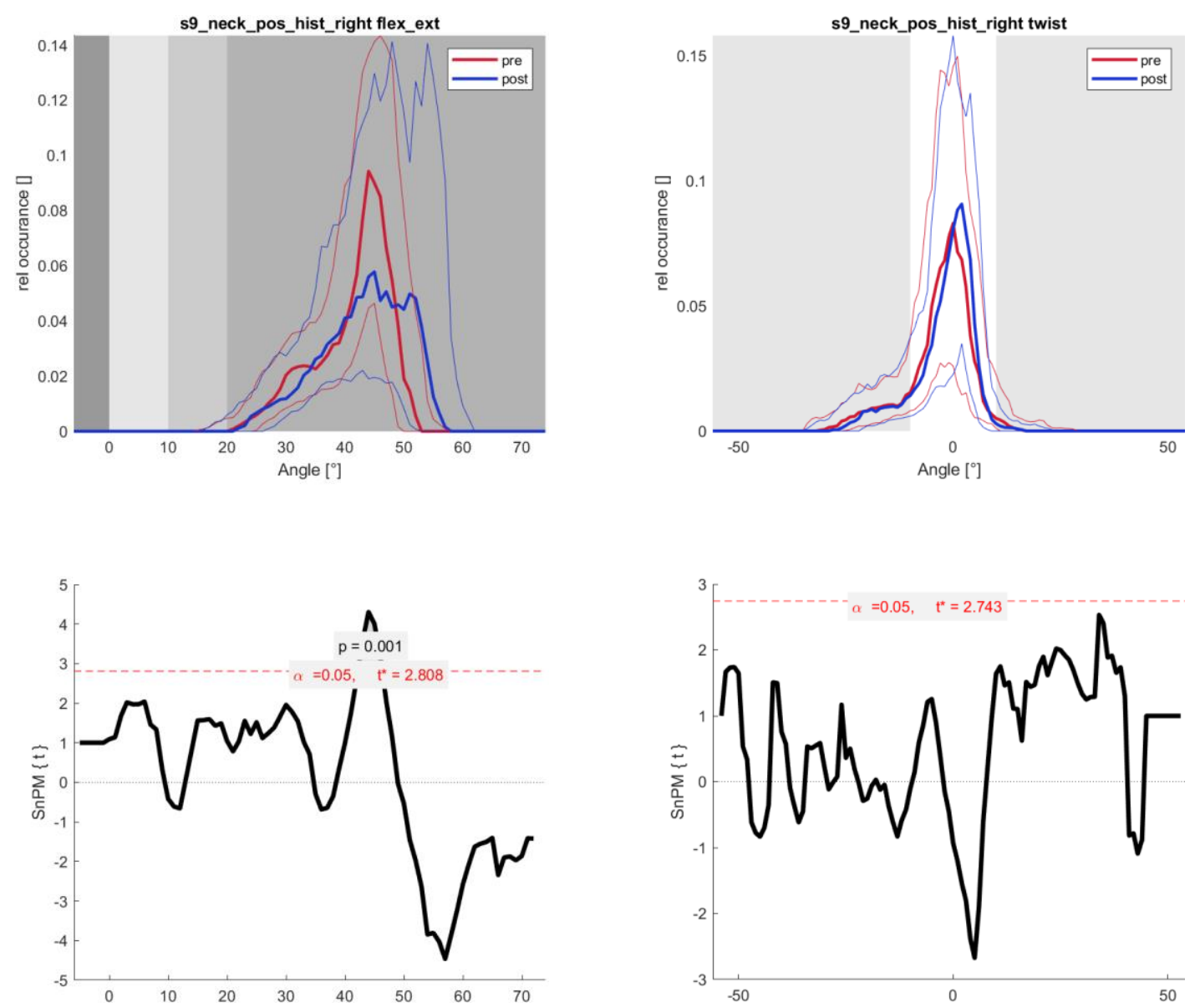

Abbildung 63: Gelenkwinkel Nacken - Flexion/Extension

Oben: Abszisse = Gelenkwinkel (positive Gradzahlen $=$ Flexion, negative Gradzahlen $=$ Extension $) ;$ Ordinate $=$ rel. zeitl . Vertei lung $($ Gesamtzeit $=1)$; Graustufen $=$ RULA Scores aufsteigend von hell (niedriger Score) nach dunkel (hoher Score) hinterlegt Unten: Abzisse $=$ Gelenkwinkel; Ordinate $=$ $t$-Werte, rote Linie $=$ Signifikanzgrenze

Abbildung 64: Gelenkwinkel Nacken - Rotation

Oben: Abszisse = Gelenkwinkel (positive Gradzahlen = Rechtsrotation, negative Gradzahlen = Linksrotation); Ordinate = rel. zeitl. Verteilung (Gesamtzeit $=1)$; Graustufen $=$ RULA Scores aufsteigend von hell (niedriger Score) nach dunkel (hoher Score) hinterlegt

Unten: Abzisse = Gelenkwinkel; Ordinate = $t$-Werte, rote Linie $=$ Signifikanzgrenze 

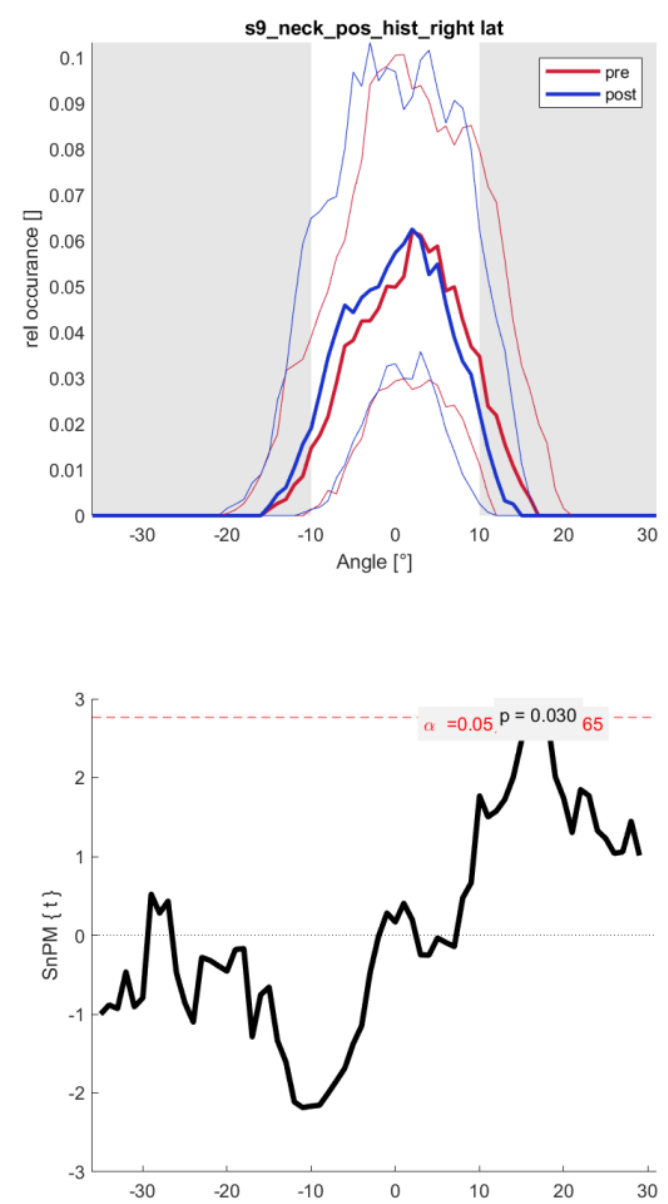

Abbildung 65: Gelenkwinkel Nacken - Beugung (Frontalebene)

Oben: Abszisse = Gelenkwinkel (positive Gradzahlen = Rechtsbeugung, negative Gradzahlen = Linksbeugung); Ordinate = rel. zeitl. Verteilung $($ Gesamtzeit $=1)$; Graustufen $=$ RULA Scores aufsteigend von hell (niedriger Score) nach dunkel (hoher Score) hinterlegt

Unten: Abzisse $=$ Gelenkwinkel; Ordinate $=t$-Werte, rote Linie $=$ Signifikanzgrenze

4.4.8 Prä-Post-Auswertung der Gelenkwinkel nach relativer zeitlicher Verteilung - Oberkörper

Für die Oberkörperregion wurden drei unterschiedliche Gelenkwinkel und entsprechende Bewegungsmuster ausgewertet. Abb. 66 zeigt die Flexion/Extension des Oberkörpers. Statistisch zeigte sich im Gelenkwinkelbereich um $15^{\circ}$ eine signifikanter $(p \leq 0,01)$ Unterschied zwischen den beiden Messungen; dieser wurde deutlich öfter während der Postmessung verzeichnet. Die stat. Signifikanz lag hier bei $p=0,001$. Es ließen sich ebenso deskriptive Unterschiede beschreiben. In beiden Messungen arbeiteten die Behandler größtenteils in einem Gelenkwinkelbereich zwischen $5^{\circ}$ und $25^{\circ}$. Somit behandeln sie mit einer leichten Flexion des Oberkörpers. In der Prämessung wurde relativ am Häufigsten 
ein Gelenkwinkel im Bereich um $18^{\circ}$ gemessen. Während der Postmessung lag der Gelenkwinkelbereich, welcher am häufigsten eingenommen wurde, um $10^{\circ}$ und somit in Richtung einer neutralen Oberkörperhaltung verschoben.

Die in Abb. 67 dargestellten Gelenkwinkel der Rotation zeigen für die Messungen vor und nach dem Trainingszeitraum nur eine marginale Varianz. Statistisch konnte kein signifikanter Unterschied ( $\mathrm{p} \geq 0,05)$ zwischen Prä- und Postmessung beschrieben werden. Die Behandler arbeiteten in beiden Messungen größtenteils in einem Gelenkwinkelbereich von $-10^{\circ}$ bis $+10^{\circ}$ mit der höchsten relativen Häufigkeit im Bereich um $0^{\circ}$. Somit arbeiteten sie zumeist in einem neutralen Rotationsbereich des Oberkörpers.

Abb. 68 zeigt die Beugung des Oberkörpers in der Frontalebene. Statistisch konnte ein signifikanter Unterschied ( $\mathrm{p} \leq 0,05)$ zwischen den beiden Messungen für die relative Häufigkeit der Gelenkwinkel im Bereich um $5^{\circ}$ gezeigt werden. Dieser wurde während der Postmessung öfter gemessen. Es lassen sich deskriptiv geringe Varianzen beschreiben. In beiden Messungen behandelten die Probanden zum Großteil in einem Gradbereich der Gelenkwinkel zwischen $-5^{\circ}$ und $+10^{\circ}$. Sie sind somit verstärkt nach rechts gebeugt. Die höchste relative Häufigkeit wurde für einen Gelenkwinkel um $2^{\circ}$ gemessen. Die blau Mediankurve der Postmessung ist dabei geringfügig in Richtung positiver Gradzahlen verschoben. 

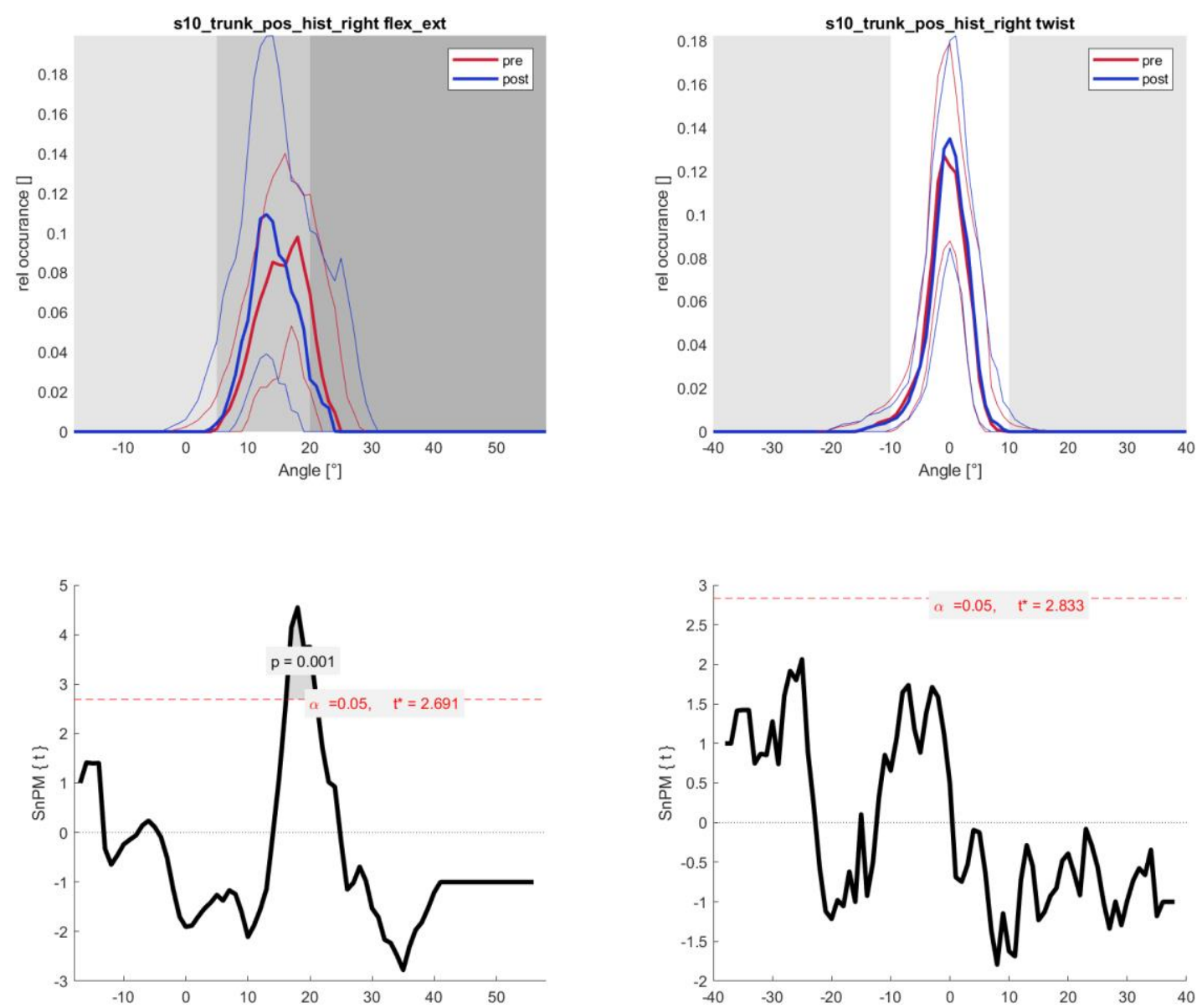

Abbildung 66: Gelenkwinkel Oberkörper Flexion/Extension

Oben: Abszisse = Gelenkwinkel (positive Gradzahlen $=$ Flexion, negative Gradzahlen $=$ Extension $) ;$ Ordinate $=$ rel. zeitl . Verte lung $($ Gesamtzeit $=1)$; Graustufen $=$ RULA Scores aufsteigend von hell (niedriger Score) nach dunkel (hoher Score) hinterlegt Unten: Abzisse $=$ Gelenkwinkel; Ordinate $=$ $t$-Werte, rote Linie $=$ Signifikanzgrenze

Abbildung 67: Gelenkwinkel Oberkörper Rotation

Oben: Abszisse = Gelenkwinkel (positive Gradzahlen $=$ Rechtsrotation, negative Gradzahlen $=$ Linksrotation $) ;$ Ordinate $=$ rel. zeitl. Verteilung (Gesamtzeit $=1)$; Graustufen $=$ RULA Scores aufsteigend von hell (niedriger Score) nach dunkel (hoher Score) hinterlegt

Unten: Abzisse = Gelenkwinkel; Ordinate = $t$-Werte, rote Linie $=$ Signifikanzgrenze 

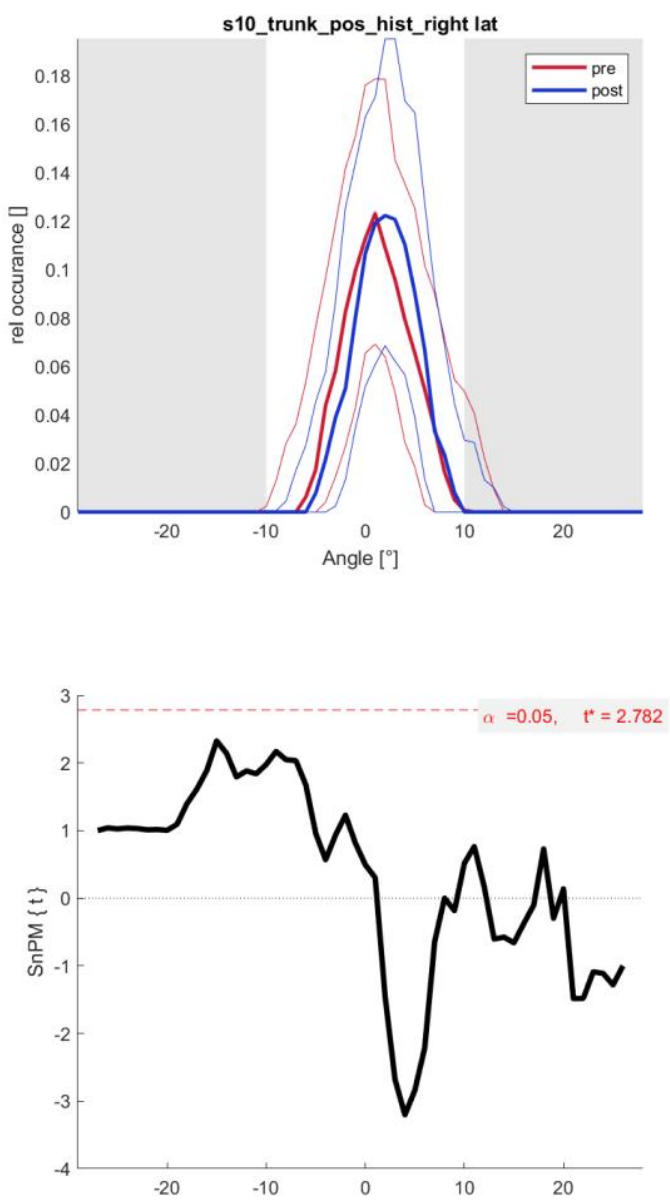

Abbildung 68: Gelenkwinkel Oberkörper - Beugung (Frontalebene)

Oben: Abszisse = Gelenkwinkel (positive Gradzahlen = Rechtsbeugung, negative Gradzahlen = Linksbeugung); Ordinate = rel. zeitl. Verteilung $($ Gesamtzeit $=1)$; Graustufen $=$ RULA Scores aufsteigend von hell (niedriger Score) nach dunkel (hoher Score) hinterlegt

Unten: Abzisse $=$ Gelenkwinkel; Ordinate $=t$-Werte, rote Linie $=$ Signifikanzgrenze 


\section{Diskussion}

\subsection{Die ergonomische Arbeitsweise von Zahnärzten und Zahnmedizinischen Fach- angestellten}

5.1.1 Ergebnisanalyse: Ergonomische Arbeitsweise von Z̈̈ und ZFAs anhand der relativen zeitlichen Verteilung von Gelenkwinkeln und Gelenkpositionen

In diesem Kapitel wird die ergonomische Arbeitsweise von ZÄ und ZFAs anhand der in Kapitel 4.4 dargestellten Ergebnisse analysiert. Für die folgende Analyse wurden die Ergebnisse der Prä- und Postmessungen aller Probanden gemeinsam betrachtet. Die mit Hilfe der Inertialsensoren durchgeführten kinematischen Messungen liefern präzise objektive Daten, in welchem Gelenkwinkel bzw. in welcher Gelenkposition die Behandler während der durchgeführten Behandlungssimulationen arbeiteten. Es sind dabei zum einen Aussagen darüber möglich, in welchem Bereich Gelenkwinkel und -positionen während der Behandlung von Z̈̈ und ZFAs eingenommen werden. Zum anderen lässt sich analysieren welche Gelenkwinkel und -positionen am häufigsten eingenommen wurden bzw. wie oft bestimmte Gelenkwinkel und -positionen in der relativen zeitlichen Verteilung gemessen wurden.

Für die Flexion bzw. Extension des Nackens konnte gezeigt werden, dass die Probanden in einem Gelenkwinkelbereich zwischen $20^{\circ}$ und $55^{\circ}$ arbeiteten. Am häufigsten wurde zwischen $30^{\circ}$ und $40^{\circ}$ gearbeitet. Eine neutrale Nackenhaltung entspricht z.B. nach den Kriterien des RULA Arbeitsbogens dem Bereich zwischen $0^{\circ}$ und $10^{\circ}$. Somit nahmen die Probanden Gelenkwinkel im Bereich zwischen moderater bis sehr starker Nackenflexion ein und arbeiteten zumeist mit stark vorgebeugtem Kopf. Die Rotation des Nackens lag während der Behandlung in einem Bereich zwischen $-20^{\circ}$ und $+10^{\circ}$. Am häufigsten wurde ein Gelenkwinkel um $0^{\circ}$ gemessen. Die Probanden behandelten folglich zum Großteil in einer neutralen Position. Der Nacken wurde i.d.R. nur gering rotiert, wobei sich eine Tendenz erkennen lässt, dass der Kopf verstärkt zur linken Seite rotiert wurde. Bei Betrachtung der Lateralflexion, wurden hauptsächlich Gelenkwinkel zwischen $-15^{\circ}$ und $+15^{\circ}$ gemessen. Der Kopf wurde von den Probanden also zeitweise nach links oder rechts gebeugt. Am häufigsten wurde mit $0^{\circ}$ eine neutrale Haltung verzeichnet. 
In der Oberkörperregion, einschließlich oberem und unterem Rücken, arbeiteten die Probanden für die Gelenkwinkel der Flexion bzw. Extension in einem Bereich zwischen $5^{\circ}$ und $25^{\circ}$. Am häufigsten wurde ein Winkel von $15^{\circ}$ gemessen. RULA definiert eine neutrale Haltung in einem Bereich von $0^{\circ}$ bis $10^{\circ}$, ab $20^{\circ}$ spricht der Arbeitsbogen von einer starken Flexion. Die Probanden arbeiteten somit in einem Bereich zwischen neutraler Oberkörperhaltung bis zeitweise stark vorgebeugtem Oberkörper, zumeist war der Oberkörper moderat vorgebeugt. Die Rotation des Oberkörpers lag im Bereich zwischen $-10^{\circ}$ und $+10^{\circ}$, am häufigsten wurde ein Gelenkwinkel bei $0^{\circ}$ gemessenen. Somit wurde vorwiegend mit geringer oder keiner Oberkörperrotation gearbeitet. Für die Beugung seitwärts in der Frontalebene wurden Winkel im Bereich von $-5^{\circ}$ bis $+10^{\circ}$ gemessen, am öftesten wurde ein Gelenkwinkel um $2^{\circ}$ erfasst. Die Probanden arbeiteten folglich meist in einer neutralen Haltung z.T. aber mit seitwärts geneigtem Oberkörper, wobei sich eine etwas stärkere Tendenz zur Beugung nach rechts beschreiben lässt.

In der Betrachtung der Schultern und oberen Gliedmaßen definiert RULA den neutralen Bereich für Flexion bzw. Extension des Oberarms zwischen $-20^{\circ}$ und $+20^{\circ}$. $\mathrm{Ab}+45^{\circ}$ spricht das Arbeitsblatt von einer starken Flexion. Der linke Oberarm der Probanden dieser Studie nahm i.d.R. in einem Gelenkwinkelbereich zwischen $0^{\circ}$ und $30^{\circ}$ ein, zumeist wurde ein Winkel um $15^{\circ}$ gemessen. Somit lässt sich schlussfolgern, dass in einem Bereich zwischen neutraler Haltung und moderater Flexion des linken Oberarms gearbeitet wurde, zumeist aber in neutraler Haltung. Die Elevation der dazugehörigen linken Schulter war i.d.R. zwischen $-13^{\circ}$ und $-5^{\circ}$. Es lässt sich schlussfolgern, dass i.d.R. mit neutraler Schulterhaltung gearbeitet wurde. Die Abduktion des linken Oberarms in der Frontalebene lag in einem Gelenkwinkelbereich zwischen $10^{\circ}$ und $35^{\circ}$, wobei die Probanden am häufigsten in einem Gelenkwinkel um $20^{\circ}$ arbeiteten. In Anlehnung an Vignais et al. [137] wurde in eine Abduktion des Oberarms von den Untersuchern als ein Winkel von $>45^{\circ}$ definiert. Die Behandler arbeiteten folglich in der Frontalebene i.d.R. in einer neutralen Haltung bzw. mit geringgradig abduzierten Oberarmen.

Der linke Unterarm der Probanden arbeitete gewöhnlich in einem Bereich von Flexion bzw. Extension zwischen $80^{\circ}$ und $120^{\circ}$. Zumeist wurde in einem Gelenkwinkel um $100^{\circ}$ behandelt. Eine neutrale Arbeitshaltung des Unterarms beschreibt der RULA Arbeitsbogen bei $80-100^{\circ}$, welche folglich zumeist von den Probanden eingenommen wurde. Die Probanden zeigten eine klar erkennbare Tendenz, den linken Unterarm verstärkt anzuwinkeln. Die Gelenkposition lag meist im Bereich zwischen -0,2m und -0,35m, mit einer 
am häufigsten gemessenen Position um -0,25m. Folglich arbeiteten die Probanden zum Großteil der gemessenen Zeit mit ihrem linken Unterarm links außerhalb der Körpermitte. Das linke Handgelenk zeigt in den Ergebnissen, dass die Probanden in Flexion bzw. Extension in einem Gelenkwinkelbereich zwischen $-55^{\circ}$ und $-20^{\circ}$ arbeiteten, meistens in einem Winkel von $-35^{\circ}$. Im RULA Arbeitsbogen ist eine starke Flexion bei einem Wert $>-15^{\circ}$ definiert. Folglich wurde zum Großteil der Behandlungszeit mit einer starken bis sehr starken Flexion des linken Handgelenks gearbeitet. Die Deviation verweist auf Gelenkwinkel in einem Bereich zwischen $-5^{\circ}$ und $25^{\circ}$. Die Probanden arbeiteten folglich in einem Bereich zwischen leichter Ulnardeviation und verstärkter Radialdeviation.

Für den rechten Oberarm ist bei Flexion bzw. Extension erkennbar, dass die Probanden für gewöhnlich in einem Gelenkwinkelbereich zwischen $0^{\circ}$ und $60^{\circ}$ am meisten in einem Winkel von $25^{\circ}$ arbeiteten. Folglich lagen die Messwerte zwischen neutraler Haltung bis hin zu einer starken Flexion des Oberarms und zumeist bei einer moderaten Flexion. Die Elevation der rechten Schulter liegt in Gelenkwinkelbereichen zwischen $\left\langle-10^{\circ}\right.$ und $>+5^{\circ}$, am meisten wurden Gelenkwinkel um $-5^{\circ}$ gemessen. Es lässt sich schlussfolgern, dass die Probanden meist nicht mit elevierter rechter Schulter arbeiteten, aber dass z.T. eine Tendenz hin zu einer angehobenen Schulter erkennbar ist. Für die Abduktion zeigen die Ergebnisse, dass Z $\ddot{\mathrm{A}}$ und ZFAs in einem Gelenkwinkelbereich von $10^{\circ}-50^{\circ}$ arbeiteten und am häufigsten ein Gelenkwinkel von $15^{\circ}$ gemessen wurde. Somit arbeiteten die Behandler zeitweise mit abduziertem rechtem Oberarm, i.d.R. in einer neutralen Haltung. Für den rechten Unterarm lässt sich erkennen, dass die Flexion bzw. Extension während der Behandlungssimulation in einem Bereich von $50^{\circ}$ und $110^{\circ}$ lag. Am meisten wurde in einem Gelenkwinkel um $80^{\circ}$ gearbeitet. Daraus kann geschlussfolgert werden, dass die Behandler zeitweise in einer moderaten Flexion bzw. einer moderaten Extension arbeiteten. Zumeist konnte die Haltung aber als neutral betrachtet werden. Wird die Gelenkposition betrachtet, so lag diese i.d.R. zwischen $0,2 \mathrm{~m}$ bis $0,4 \mathrm{~m}$. Hier arbeiteten die Behandler also mit dem Unterarm nach links über die Körpermitte hinaus und nahmen zumeist keine neutrale Haltung ein.

Die Flexion bzw. Extension des rechten Handgelenks zeigt, dass ZÄ und ZFAs zumeist in einem Bereich zwischen $-60^{\circ}$ und $0^{\circ}$ arbeiteten und $-45^{\circ}$ der am häufigsten eingenommenen Gelenkwinkel ist. Folglich wurden Winkel zwischen einer neutralen Haltung und einer sehr starken Flexion eingenommen. Größtenteils behandelten die Probanden mit einem stark gebeugten rechten Handgelenk. Werden die Ergebnisse der Deviation des 
linken Handgelenks betrachtet, so lag diese in einem Gelenkwinkelbereich zwischen $-15^{\circ}$ und $25^{\circ}$ und zumeist wurde ein Gelenkwinkel um $5^{\circ}$ eingenommen. Folglich nahmen die Probanden zumeist eine neutrale Haltung des Handgelenks bezüglich der Deviation ein, arbeiteten aber zeitweise auch im Bereich der Radial- und Ulnarflexion mit einer verstärkten Tendenz zur Radialflexion.

In der Zahnmedizin ist die Untersuchung der ergonomischen Arbeitsweise anhand von objektiven Daten, ermöglicht durch sensorbasierte Messsysteme, bisher nur in vereinzelten Studien beschrieben.[2, 11, 134, 183] Ohlendorf et al. [2, 11] untersuchten anhand von CUELA, einem Inertialsensorsystem, das ergonomische Risiko besonders häufig ausgeführter Tätigkeiten im Tagesgeschäft von Zahnärzten. 21 Z̈̈ (11 w/10 m) wurde bei der täglichen Arbeit zur Erfassung kinematischer Daten untersucht. Dabei wurden die eingenommenen Gelenkwinkel für die Segmente Kopf/Nacken sowie Rücken untersucht. Die während der Patientenbehandlung erfassten Daten bzw. Gelenkwinkel wurden anhand der Perzentile 5, 25, 50 (Median), 75 und 95 ausgewertet. Für die Nackenregion, genauer gesagt, die Halswirbelsäule stellten die Untersucher in der Flexion bzw. Extension Medianwerte zwischen $-6^{\circ}$ und $26^{\circ}$ fest und schlussfolgerten daraus, dass die Nackenhaltung median immer in einem neutralen bis moderaten Bereich lag. Im Vergleich zur vorliegenden Studie wurden also insgesamt deutlich neutralere Gelenkwinkel gemessen. Der Abstand der jeweils gemessenen Gelenkwinkelbereiche stimmt allerdings überein und liegt jeweils bei ca. $30^{\circ}$. Die Abweichungen der gemessenen Gelenkwinkel voneinander lassen sich ggf. durch eine Platzierung der Sensoren erklären, je nach Messsystem können die jeweils vorgegebenen Positionen der Sensoren variieren. Außerdem wurden in der Vergleichsstudie nur ZÄ und keine ZFAs untersucht. Das abweichende Aufgabenfeld und die kontralaterale Positionierung der ZFAs können somit in der vorliegenden Studie zu Abweichungen geführt haben. Dennoch besteht Einigkeit darüber, dass Probanden beider Studien meist mit nach anterior gebeugtem Nacken arbeiteten. Dass wiederum die Nackenflexion von Ohlendorf et al. [2, 11] als neutral bis moderat und nicht, wie in der vorliegenden Studie, als moderat bis sehr stark bewertet wurde ist auch darauf zurück zu führen, dass die Auswertung nicht dem RULA Arbeitsbogen zugrunde lag. Die der Vergleichsstudie zugrundeliegende Auswertungsmethode Ovako Working posture Analysis (OWAS) definiert den neutralen Gelenkwinkelbereich zwischen $0^{\circ}$ und $25^{\circ}$, bei RULA hingegen liegt er zwischen $0^{\circ}$ und $10^{\circ}$ und bewertet somit konservativer. 
In der Lateralflexion wurden in der Vergleichsstudie Gelenkwinkel in einem Bereich zwischen $-5^{\circ}$ und $+5^{\circ}$ beschrieben. Somit wurde die ergonomische Arbeitsweise diesbezüglich als symmetrisch betrachtet. Die vorliegende Studie bestätigt diese Aussage, auch wenn insgesamt höhere Winkel gemessen wurden.

Für die Oberkörperregion stellten die Untersucher Gelenkwinkel im Bereich zwischen $15^{\circ}$ bis $38^{\circ}$ fest, welche als zumeist neutrale Haltungen bewertet wurden. Die Spannweite des gemessenen Gelenkwinkelbereichs der beiden Studien ist somit annähernd gleich, während in der vorliegenden Studie allerdings der gesamte Bereich um $10^{\circ}$ in Richtung $0^{\circ}$ verschoben liegt. Beide Bereiche stimmen in einem Großteil der gemessenen Winkel überein und es besteht Einigkeit darüber, dass Probanden beider Studien zumeist mit vorgebeugtem Oberkörper arbeiteten. Auch hier lassen sich die Abweichungen anhand der abweichenden Sensorpositionierung erklären. In ihrer Bewertung stimmen beide Studien wieder darin überein, dass die Probanden zumeist in neutralen Gelenkwinkeln und zeitweise mit einer verstärkten Flexion bezüglich des Oberkörpers arbeiteten. Bezüglich der Lateralflexion des Oberkörpers beschrieben Ohlendorf et al.[2, 11] einen Gelenkwinkelbereich zwischen $-7^{\circ}$ und $+15^{\circ}$ und schlussfolgerten daraus, dass die Probanden zwar größtenteils im neutralen Bereich arbeiteten allerdings mit verstärkter Inklination nach rechts. Der gemessene Gelenkwinkelbereich der vorliegenden Studie stimmt weitgehend mit der Vergleichsstudie überein und auch die Schlussfolgerung einer verstärkten rechtsseitigen Lateralflexion wird bestätigt.

Trotz der Unterschiede bezüglich Messsystem, Auswertungssystem, Probandenkollektiv und Aufgabenstellung lässt sich zusammenfassend schlussfolgern, dass die ergonomische Arbeitsweise, wie sie von Ohlendorf et al. [2, 11] bereits untersucht und beschrieben wurde, durch die Ergebnisse dieser Studie bestätigt wird. Der Oberkörper ist zumeist gekippt bzw. vorgebeugt, während es verstärkt zu einer leichten Inklination nach rechts kommt. Der Nacken ist ebenfalls vorgebeugt und zeitweise rotiert, um eine bessere Einsicht in die Mundhöhle zu ermöglichen. Die in beiden Studien für bestimmte Segmente gemessenen ungleichen Winkelbereiche weisen auf eine asymmetrische Arbeitshaltung hin. 
5.1.2 Die ergonomische Arbeitsweise unter Berücksichtigung ergonomischer Vorgaben aus der Zahnmedizin

Im Folgenden wird, die oben analysierte, ergonomische Arbeitsweise der Probanden dieser Studie verglichen mit den in Kapitel 2.5 vorgestellten Vorgaben für eine ideale Ergonomie in der Zahnmedizin.

Die ISO 11226 [20] dient oftmals als Grundlage von der ergonomische Standards in der Zahnmedizin abgeleitet werden.[21-24] Sie findet Anwendung bei Berufen mit statischen Arbeitshaltungen. Dabei gibt sie als Ideal eine sitzende, aktive, aufrechte und symmetrische Arbeitshaltung vor. Die Probanden dieser Studie arbeiteten alle während der Behandlungssimulation im Sitzen. Wie bereits im vorherigen Kapitel ausgeführt, lässt sich die Körperhaltung der Probanden nicht als aufrecht beschreiben, sondern ist gekennzeichnet durch eine nach anterior gebeugte Haltung des Oberkörpers und Rückens. Neben ungleichen Winkelbereichen einzelner Körpersegmente (z.B. Lateralflexion Oberkörper) fällt auch im Seitenvergleich der oberen Extremitäten eine Asymmetrie der Haltung auf. Der rechte Oberarm zeigt eine stärkere Flexion sowie Abduktion. Außerdem wurde die rechte Schulter von den Probanden tendenziell stärker eleviert. Diese Tendenzen des rechten Oberarms hin zu einer belastenderen ergonomischen Arbeitsweise außerhalb neutraler Gelenkwinkel lassen sich vermutlich dadurch erklären, dass die Behandler dominant rechtshändig behandelten. Zum Erreichen der Mundhöhle für die Durchführung der zahnärztlichen Aufgaben war dementsprechend eine erhöhte Mobilität des rechten Arms notwendig.

Im Vergleich mit speziell für die Zahnmedizin gemachten Vorgaben bezüglich einer optimalen ergonomischen Arbeitsweise wird deutlich, dass auch von diesen z.T. deutlich abgewichen wird. Diverse Autoren [21-25, 97, 99] beschreiben eine optimale ergonomische Arbeitshaltung. Ihre Vorgaben werden im Folgenden kurz zusammengefasst, um dann die abweichenden Ergebnisse dieser Studie gegenüber zu stellen. Der Oberkörper soll neutral gehalten werden und maximal $10^{\circ}$ bis $20^{\circ}$ von der Hüfte vorgebeugt werden [21, 25, 99]. Der Kopf sollte wenn möglich in einer neutralen Haltung bleiben; maximal aber $20^{\circ}$ vorgebeugt sein. [21, 23-25, 99] Des Weiteren sollte eine Lateralflexion und Rotation von Oberkörper und Nacken vermieden werden [21, 23-25, 99]. Bezüglich der oberen Extremitäten sollte der Oberarm neutral gehalten werden und dem Oberkörper anliegen, wobei die Schultern ebenfalls in neutraler Position bleiben; maximal soll in Flexion/Extension einen Gelenkwinkel von $25^{\circ}$ eingenommen werden. In der Frontalebene 
soll der Oberarm maximal 15-20 abduziert sein.[21-23, 25] Die Unterarme sollten idealerweise parallel zum Boden gehalten werden und einen Winkel zum Oberarm zwischen $70^{\circ}$ und $100^{\circ}$ einnehmen. [21-25, 99] Die Handgelenke sollen entspannt gehalten werden und möglichst nicht angewinkelt oder rotiert sein.

Die Probanden dieser Studie arbeiten meistens mit vorgebeugtem Oberkörper im akzeptablen Bereich; maximal wurden die Vorgaben um bis zu ca. $5^{\circ}$ überschritten. Die Flexion des Nackens war fast ausschließlich $>20^{\circ}$ und zumeist zwischen $30^{\circ}$ bis $40^{\circ}$ (maximal $55^{\circ}$ ). Somit wurde der Kopf größtenteils deutlich weiter vorgebeugt als empfohlen. Bezüglich Rotation und Lateralflexion von Oberkörper und Nacken bezüglich Rotation und Lateralflexion von Oberkörper und Nacken wichen die Probanden nur einen geringen Anteil der Zeit von den empfohlenen neutralen Gelenkwinkeln ab (maximal bis zu $10^{\circ}$ in Lateralflexion bzw. $20^{\circ}$ in Rotation). Bezüglich der oberen Extremitäten arbeiteten die Probanden auf der linken Seite zumeist mit dem Oberarm im neutralen Gelenkwinkelbereich, vereinzelt auch außerhalb mit $>25^{\circ}$. Durchschnittlich wurde der Arm nicht zu stark vom Oberkörper abduziert. Maximal erreichte Gelenkwinkeln bis zu $35^{\circ}$ lagen allerdings z.T. weit außerhalb der empfohlenen Körperhaltung. Die linke Schulter wich i.d.R. nicht von den Empfehlungen ab. Auf der rechten Seite behandelten die Probanden am häufigsten mit einer Flexion des Oberarms von ca. $25^{\circ}$ und somit an der Grenzschwelle der ergonomischen Empfehlungen. Einen großen Anteil der Messzeit arbeiteten sie aber auch in Gelenkwinkeln $>25^{\circ}$ bis $60^{\circ}$ und wichen so deutlich vom empfohlenen Gelenkwinkelbereich ab. In der Frontalebene entsprach die Abduktion durchschnittlich den empfohlenen Gelenkwinkeln, maximal allerdings abduzierten die Probanden den deutlich stärker bis zu einem Gelenkwinkel von 50 . Die Probanden zeigten auf der rechten Seite eine Tendenz zu einer zeitweiligen Elevation der Schulter. Die Flexion des linken Unterarms lag meist in einem Gelenkwinkelbereich um $100^{\circ}$ und somit bereits am oberen Ende der zahnärztlichen Empfehlungen. Einen großen Teil der Zeit arbeiteten sie allerdings auch in Gelenkwinkelbereichen $>100^{\circ}$ bis $120^{\circ}$. Mit dem rechten Unterarm arbeiteten die Probanden zumeist im empfohlenen Bereich: maximal wurden Gelenkwinkel bis $110^{\circ}$ gemessen. Das linke Handgelenk arbeitete fast ausschließlich in einer Flexion und folglich nicht in der geforderten neutralen Position. Die Probanden arbeiteten mit deutlich bis stark angewinkeltem Handgelenk (maximal $-55^{\circ}$ ). Außerdem konnte auf der linken Seite zumeist eine Deviation des Handgelenks festgestellt werden (maximal $25^{\circ}$ ). Auf der rechten Seite konnte bei den Probanden eine i.d.R. noch stärkere Flexion des Handgelenks 
beschrieben werde (maximal $-60^{\circ}$ ). Auch hier zeigte das Handgelenk zum Großteil der Zeit eine Deviation und keine neutrale Haltung (maximal 25).

Zusammenfassend lässt sich für den Vergleich zwischen zahnmedizinischen Vorgaben für die ergonomische Arbeitsweise [16, 20-25, 67, 97] und die in dieser Studie anhand objektiver Daten evaluierten tatsächlichen ergonomischen Arbeitsweise sagen, dass die ZÄ und ZFAs nur selten die Anforderungen einer optimalen Arbeitsweise erfüllen. Zwar arbeiten die Probanden bezüglich mancher Gelenkwinkeln meist in empfohlenen Winkelbereichen, wie z.B. für die Flexion des Oberkörpers, die Rotation und Lateralflexion des Nackens oder die Flexion von rechtem und linkem Oberarmarm. Allerdings ist fast immer eine zeitweilige Abweichung zu beschreiben. Am weitesten weichen die Probanden bezüglich der Flexion des Nackens, der Flexion des rechten Oberarms sowie für die Flexion und Deviation der beiden Handgelenke ab. Es wird angenommen, dass das das Risiko MSE zu entwickeln umso größer ist, je stärker ein Gelenkwinkel von seiner neutralen Haltung abweicht.[97] Somit lässt sich für die untersuchten Berufsgruppen herleiten dass sie einem hohem gesundheitliches Risiko ausgesetzt ist MSE zu entwickeln. Dieses Risiko wird im folgenden Kapitel diskutiert.

5.1.3 Die ergonomische Arbeitsweise unter Berücksichtigung des Berufsbilds von Zahnärzten und Zahnmedizinischen Fachangestellten

Das Berufsbild von ZA und ZFA und die mit ihm verbundenen beruflichen gesundheitlichen Risikofaktoren sind in Kapitel 2.3 umfassend beschrieben und die mit ihnen verbundenen gesundheitlichen Folgen [3, 4, 7, 11-13, 15, 18, 67, 69] . In diesem Kapitel werden die bereits erforschten gesundheitlich riskanten Arbeitsweisen mit der in dieser Studie analysierten ergonomischen Arbeitsweise von Z̈̈ und ZFA verglichen.

In der zahnmedizinischen Literatur [3, 4, 12, 13] wird oftmals beschrieben, dass die Behandler eine krumme, vorgebeugte Haltung mit runden Schultern einnehmen, da ihr Arbeitsfeld (der Patientenmund) zumeist unter ihnen liegt und zudem klein und schlecht einsehbar ist. Marklin und Cherney [67] untersuchten die Arbeitshaltung von $10 \mathrm{ZÄ}$ und 10 ZFAs jeweils vier Stunden lang während der Patientenbehandlung anhand von Videoaufnahmen. analysiert wurde die Haltung des Oberkörpers sowie der Nackenregion wobei die eingenommenen Haltungen kategorisiert wurden in $30^{\circ}$ Intervallen: $0^{\circ}$ (neutrale Gelenkhaltung), $\geq 30^{\circ}, \geq 60^{\circ}, \geq 90^{\circ}$. Anhand der Kategorien wurde die zeitliche Verteilung der eingenommenen Haltung für das jeweilige Gelenk berechnet. Dabei stellten sie fest, 
dass beide Professionen über die Hälfte ihrer Arbeitszeit mit einem vorgebeugten Oberkörper um mindestens $\geq 30^{\circ}$ verbrachten. In der vorliegenden Studie konnte anhand der objektiven Daten gezeigt werden, dass die Probanden in ausschließlich in einem Gelenkwinkelbereich zwischen $5^{\circ}$ und $25^{\circ}$, am häufigsten mit einer Ventralflexion des Oberkörpers von circa $15^{\circ}$. Die Probanden dieser Studie weichen insgesamt also weniger von einer neutralen Position ab als in der Vergleichsstudie und arbeiten zeitlich nie im Bereich $\geq 30^{\circ}$. Die Abweichungen könnten sich allerdings dadurch erklären lassen, dass in der Vergleichsstudie nicht mit objektiven Daten gearbeitet wurde. Das Bewerten des Gelenkwinkels unterlag somit der subjektiven Einschätzung der Untersucher, welcher diese ggf. nicht richtig erfassen konnte oder ihn zu hoch einschätzten. Marklin und Cherney [67] beschrieben außerdem in der gleichen Studie, dass beide Professionen 85\% ihrer Arbeitszeit mit einem vorgebeugten Nacken um mindestens $\geq 30^{\circ}$ behandelten. Die objektiven Daten der vorliegenden Studie zeigten ebenfalls, dass die Behandler den Großteil der Zeit mit einer Flexion des Nackens zwischen $35^{\circ}$ bis $45^{\circ}$ arbeiteten, zeitweise auch mit deutlich stärker vorgebeugtem Nacken bis zu $55^{\circ}$. In diesem Fall stimmen die Einschätzungen von Marklin und Cherney [67] folglich weitgehend mit den Ergebnissen dieser Studie überein. Morse et al. [12] untersuchten 160 ZFAs und Auszubildene bezüglich ihrer Selbsteinschätzung von Nacken- und Schulterbeschwerden und biomechanischen Risikofaktoren des Berufsfelds. In diesem Zusammenhand wurde beschrieben, dass über $90 \%$ der erfahrenen ZFAs Nackenbeugen als beruflichen Risikofaktor für die Entwicklung von MSE ansehen.

Zusätzlich zum Vorbeugen des Oberkörpers beschreibt die Literatur [3, 4, 11, 12, 69] außerdem, dass ZÄ und ZFAs für eine bessere Einsicht in die Mundhöhle und um die Präzisionsanforderungen ihres Berufes zu erfüllen, den Oberkörper und Nacken zusätzlich rotieren und seitlich beugen. Anhand der objektiven Daten lässt sich zweifellos beschreiben, dass die Probanden dieser Studie regelmäßig mit rotiertem Oberkörper und ebenfalls rotiertem Nacken arbeiteten. Zwar wurden am meisten neutrale Gelenkwinkel gemessen, dennoch zeigen die Gelenkwinkelbereiche, dass regelmäßig nach rechts oder links rotiert wurde. Für den Oberkörper wurden Rotationen bis zu $10^{\circ}$, für den Nacken bis zu $20^{\circ}$ gemessen. Ähnliches gilt für die Lateralflexion der beiden Gelenke in der Frontalebene. Die Behandler beugten den Oberkörper sowie den Nacken während der Messungen nach lateral, wenn auch meist nur geringgradig. Für den Oberkörper wurden hierbei Lateralflexionen bis zu $10^{\circ}$ und für den Nacken bis zu $15^{\circ}$ gemessen. Morse et al. [12] 
beschrieben in der bereits angeführten Studie, dass über 70\% der erfahrenen ZFAs Nackenrotation als beruflichen Risikofaktor betrachten. In Folge von Torsion, Flexion sowie Lateralflexion des Rumpfes kann es wiederum zu einer Störung des muskulären Gleichgewichts kommen.[3, 4, 10, 12, 29]

In der Literatur $[3,7,12,15]$ ist des Weiteren beschrieben, dass während der Behandlung ZÄ und ZFAs oftmals über lange Zeit mit angehobenem und abduziertem Arm arbeiten müssen. Beispielsweise um einen Spiegel, Sauger oder ein anderes Instrumentarium zu halten, damit eine Behandlung unter übersichtlichen Bedingungen möglich ist. Finsen et al. [7] beschrieben in ihrer Studie, die die Arbeitshaltung von Zahnärzten anhand der drei üblichsten Arbeitsaufgaben untersuchte, dass Behandler lange Perioden mit erhobenem Arm und ungefähr ein Drittel der Arbeitszeit mit einer Abduktion des Armes über $30^{\circ}$ arbeiteten. Die Ergebnisse dieser Studie zeigen, dass die Probanden linksseitig mit einer Flexion des Oberarms bis zu $30^{\circ}$ arbeiteten. Am häufigsten wurden $15^{\circ}$ gemessen. Die kinematischen Daten zeigen allerdings keine Elevation der linken Schulter. Für die linke Seite lässt sich folglich sagen, dass die Probanden dieser Studie zeitweise, meist aber nicht mit angehobenem Arm arbeiteten. In der Abduktion des linken Oberarms arbeiteten die Probanden in Gelenkwinkeln $>30^{\circ}$; am meisten wurde ein Gelenkwinkel von $18^{\circ}$ gemessen. Es lässt sich demnach eine ähnliche Tendenz zu den von Finsen et al.[7] beschriebenen Ergebnissen erkennen. Für den rechten Oberarm zeigen die Ergebnisse dieser Studie, dass die Probanden rechtsseitig mit einer Flexion bis zu $60^{\circ}$ arbeiten; am häufigsten wurde ein Gelenkwinkel um $25^{\circ}$ gemessen. Für die rechte Schulter ist außerdem anhand der kinematischen Daten die Tendenz einer Elevation erkennbar. Rechtsseitig arbeiteten die Probanden folglich öfters und länger mit angehobenem Oberarm. In der Abduktion des rechten Oberarms behandelten die Z̈̈ und ZFAs in Gelenkwinkeln bis zu $50^{\circ}$ und dementsprechend lange in einem Bereich mit stärker abduziertem Oberarm. Es lässt sich festhalten, dass die von Finsen et al .[7] beschriebene Arbeitsweise mit den Ergebnisse dieser Studie besonders auf der rechten Seite übereinstimmt, während sich auf der linken Seite eine ähnliche Tendenz erkennen lässt. Diese Arbeitsweise und die verbundene Arbeitsbelastung stehen in engem Zusammenhang zu erhöhten MSE-Prävalenzen, zum Beispiel zum vermehrten Auftreten von Schulterproblemen.[3, 7, 15] Zusammenfassend lässt sich sagen, dass die ergonomische Arbeitsweise der Probanden dieser Studie sich zum Großteil in Einklang bringen lässt mit dem Berufsbild und den beruflichen Risiken des Arbeitsumfelds, wie sie andere Studien [3, 4, 7, 11-13, 15, 18, 
67, 69] beschreiben. Die erhobenen Gelenkwinkel stimmen oft mit denen von anderen Autoren [3, 4, 7, 11, 13, 67, 69] als gesundheitlich riskant definierten Arbeitsweisen von ZÄ und ZFAs überein. Die in dieser Studie erhobenen objektiven Daten zeichnen ein höchstpräzises Bild der ergonomischen Arbeitsweise von Z̈̈ und ZFAs. Anhand des inertialsensorbasierten direkten Messssystems XSens, konnten die ergonomische Arbeitsweise der Probanden ganzheitlich und über den gesamten Behandlungszeitraum hinweg evaluiert werden, ohne den subjektiven Einfluss der Untersucher. In den verglichenen Studien [7, 67] erfolgte das Erfassen der ergonomischen Arbeitsweise zumeist durch die Beobachtungen des Untersuchers, welcher in seiner Kapazität limitiert ist den gesamten Behandlungsverlaufs objektiv zu erfassen oder z.B. schwer einsehbare Körperhaltungen (z.B. Handgelenke, Rumpfrotation) zu beurteilen (siehe Kapitel 5.2.2).[119, 129] Das beschriebene hohe gesundheitliche Risiko für die zahnmedizinischen Berufe kann somit anhand dieser präzisen experimentellen, objektiven Daten bestätigt werden. Die in Kapitel 2.3 beschriebenen gesundheitlichen Folgen sind dementsprechend auch potenzielle Gefahren für die Probanden dieser Studie.

\subsection{Die ergonomische Risikobeurteilung von Zahnärzten und Zahnmedizinischen Fachangestellten}

5.2.1 Ergebnisanalyse: ergonomische Risikobeurteilung von ZÄ und ZFAs anhand RULA Scores und ihrer relativen zeitlichen Verteilung

In diesem Kapitel wird die ergonomische Risikobeurteilung von ZÄ und ZFAs anhand der in Kapitel 4.1 bis 4.3 dargestellten Ergebnisse analysiert. Die Ergebnisse der Prä- und Postmessungen aller Probanden wurden erneut gemeinsam betrachtet. Die Bewertung mit RULA Scores und die relative zeitliche Verteilung der Bewegungsparameter lassen eine differenzierte Einschätzung des gesundheitlichen Risikos der ergonomischen Arbeitsweise zu.[120] Die folgende Analyse beginnt allgemein und wird dann für die einzelnen Regionen spezifisch.

Der RULA Gesamtscore lässt, im Gegensatz zu den Teilscores der einzelnen Regionen, eine im RULA Arbeitsbogen beinhaltete Handlungsempfehlung zu, die dem Untersucher 
bzw. Untersuchten dabei unterstützt die Ergebnisse adäquat zu interpretieren. Die ergonomische Arbeitsweise der Probanden dieser Studie erhielt im Median einen RULA Gesamtscore von 7, der höchsten erreichbaren Punktzahl. Nach dem RULA Arbeitsbogen soll eine solche ergonomische Arbeitsweise weiteren Untersuchungen unterzogen werden und außerdem so bald wie möglich geändert werden, da sie ein sehr hohes gesundheitliches Risiko darstellt.[120] Wird die relative zeitliche Verteilung des RULA Gesamtscores betrachtet, wird deutlich, dass die Probanden knapp 80\% im höchsten erreichbaren Score 7 und circa 20\% in den Scores 5 und 6, welche nach dem RULA Arbeitsbogen ebenfalls mit einem hohen gesundheitlichen Risiko assoziiert sind, weshalb eine weitere Untersuchung sowie baldige Änderung der Arbeitsweise empfohlen werden.

Die Evaluation des ergonomischen Risikos von Z̈̈ und ZFAs ist bereits durch mehrere Autoren [29, 121-123] in zahnmedizinischen Studien beschrieben worden. Einige dieser Studien $[29,121,123]$ nutzten ebenfalls für die ergonomische Risikobeurteilung die klassische, observatorische Methode von RULA und lassen dadurch eine erhöhte Vergleichbarkeit zu. Rafie et al.[29] untersuchten 130 ZÄ während ihrer sitzenden Arbeit am Patienten für 20 Minuten. Die Bewertung der ergonomischen Arbeitsweise erfolgte mit RULA, wobei allerdings lediglich die von den Probanden als dominant angegebene Körperhälfte bewertet wurde. Die Ergebnisse des RULA Gesamtscores zeigte, dass 18.2\% der Probanden mit RULA Score 3 und 4, 57\% RULA Score 5 und 6 sowie 24,8\% RULA Score 7 bewertet wurden. Im Vergleich zu den Ergebnissen dieser Studie, in der annähernd alle Probanden einen RULA Gesamtscore von 7 hatten, zeigt sich, dass Rafie et al. [29]die ergonomische Arbeitsweise insgesamt als weniger gesundheitlich riskant einschätzen. Die Autoren selbst kommen dennoch zum Schluss, dass inkorrekte ergonomische Gewohnheiten und Arbeitsweisen unter Z $\ddot{A}$ stark verbreitet sind, welche mit der hohen Prävalenz von MSE in dieser Berufsgruppe assoziiert sind. Diese Schlussfolgerung lässt sich anhand der Ergebnisse dieser Studie bestätigen. Es lässt sich festhalten, dass auch die Vergleichsstudie die Arbeitsweise von keinem Probanden als akzeptabel einschätzte, sondern der RULA Arbeitsbogen immer eine weitere Untersuchung sowie potenzielle oder definitive Änderungen der Arbeitsweise empfahl. Die Vergleichbarkeit der Studien muss allerdings relativiert werden, da in der Studie von Rafie et al.[29] nur die ergonomische Arbeitsweise der dominanten Körperseite untersuchten. In der vorliegenden Studie hingegen wurde die ergonomische Arbeitsweise des gesamten Körpers bewertet, da trotz einer dominanten Seite ZÄ und ZFAs oft während der Behandlung beidhändig 
arbeiten müssen, um Einsehbarkeit und Präzision zu gewährleisten (Bsp. Z ̈̈: rechte Hand Winkelstück, linke Hand Spiegel). Um das ergonomische Risiko präzise zu erfassen dürfen beteiligte Körperregionen somit nicht aus der Bewertung genommen werden. Choobineh et al. [123] untersuchten die ergonomische Arbeitsweise des gesamten Körpers mittels RULA an 139 ZÄ. Dabei wurden während der Behandlung am Patienten eingenommene Körperhaltungen fotografiert und die repetitivsten ausgewählt um sie mit RULA zu bewertet. Wie die repetitivsten Körperhaltungen ausgewählt wurden wird von den Autoren nicht spezifiziert. Als Ergebnisse wurden ein RULA Gesamtscore für die linke Kör-

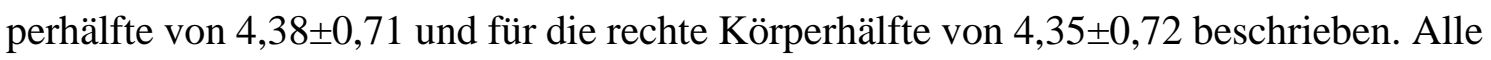
Probanden lagen in einem RULA Gesamtscore zwischen 3 und 6. Die ergonomische Arbeitsweise der $\mathrm{ZÄ}$ wurde entsprechend wiederum als weniger riskant bewertet als in dieser Studie, doch auch hier wurde keine ergonomische Arbeitsweise als akzeptabel bewertet. Die abweichende Methodik der Untersuchung schränkt die Vergleichbarkeit allerdings ein, was in einem folgenden Abschnitt dieses Kapitels (Kapitel 5.2.2) noch diskutiert wird. Auch Choobineh et al.[123] schlussfolgerten, dass das Z ̈̈ somit einem hohen ergonomischen Risiko ausgesetzt sind und ein hohes Risiko haben MSE zu entwickeln. In den angeführten Studien wurde dabei nur der RULA Gesamtscore nicht aber die RULA Teilscores der jeweiligen Probanden in ihre Analysen hinzugezogen. Die Analyse der einzelnen Teilscores kann allerdings Aufschluss darüber geben, welche Körperregionen als besonders riskant bewertet werden und somit u.U. einen besonderen Beitrag zum RULA Gesamtscore bzw. gesundheitlichen Risiko leisten. Wird in der vorliegenden Studie der RULA Teilscore der Oberkörperregion betrachtet, so lag der Medianwert der Probanden für diesen RULA Score bei 2 von 6 erzielbaren Punkten. In Anlehnung an die Arbeitsgruppe von Vignais et al. [137] wurden auch für die Teilscores Risikoschwellen definiert, ab denen die ausgewerteten Scores als gesundheitlich riskant gelten. Für die Oberkörperregion war die Risikoschwelle bei einem Score $\geq 4$ definiert. Unter Betrachtung der relativen zeitlichen Verteilung lässt sich erkennen, dass die Probanden nur gute $2 \%$ der gemessenen Arbeitszeit im RULA Score 4 und 0\% im jeweiligen RULA Score 5 und 6 verbrachten. Somit ist die Arbeitsweise insgesamt nicht als gesundheitsschädlich einzuschätzen.

Der RULA Teilscore der Nackenregion, wurde im Median mit 3 von 6 Punkten bewertet (Risikoschwelle Score $\geq 4$ ). Das obere Quartil lag allerdings zwischen den Scores 3,5 und 4 und auch in der relativen zeitlichen Verteilung der RULA Scores wird deutlich, dass 
die Probanden über $40 \%$ der Zeit in gesundheitlich riskant bewerteten Scores arbeiteten. Die ergonomische Arbeitsweise der Probanden ist folglich insgesamt noch nicht gesundheitsschädlich eingestuft, allerdings arbeiten die Probanden bereits fast ausschließlich direkt unterhalb oder bereits oberhalb der definierten Risikoschwelle.

Der Medianwert des RULA Teilscores für den Oberarm linksseitig lag zwischen 1 und 1,5 von 6 möglichen Punkten. Auch die relative zeitlich Verteilung zeigt, dass die Probanden nicht in den gesundheitlich riskanten Scores $\geq 5$ arbeiteten. Es ist von einer ergonomisch akzeptablen Arbeitshaltung auszugehen. Die Ergebnisse für den linken Unterarm zeigen, dass der Medianwert zwischen 2 und 2,5 und somit unterhalb der Risikoschwelle (Score $\geq 3$ ) lag. Das obere Quartil lag allerdings bei 3 und auch die relative zeitliche Verteilung zeigt, dass die Probanden insgesamt circa 47\% der Zeit im RULA Score 3 arbeiteten. Zusammenfassend kann die ergonomische Arbeitsweise der Probanden in der Region des linken Unterarms insgesamt noch nicht als gesundheitsschädlich eingestuft werden, die Probanden behandelten aber fast ausschließlich direkt unterhalb oder bereits oberhalb der Risikoschwelle. Der Handgelenk- \& Rotations-Score linksseitig zeigt für den Medianwert 5 von 6 erreichbaren Punkten und liegt somit gleichzeitig auf der ergonomischen Risikoschwelle. Die relative zeitliche Verteilung macht erkenntlich, dass die Arbeitsweise der Probanden zu ungefähr 65\% der gemessenen Zeit als Score 5 bewertet wurden. Insgesamt ist die Arbeitsweise der Probanden in der linken Handgelenk$\&$ Rotations-Region als gesundheitlich riskant zu bewerten.

Auf der rechten Seite wurde der Oberarm im Median mit 2 von 6 Punkten bewertet. Der relativen zeitlichen Verteilung ist erkennbar, dass die Probanden in $>90 \%$ der Arbeitszeit mit einem RULA Score von 1 oder 2 bewertet wurde. Somit ist von einer ergonomisch akzeptablen Arbeitshaltung auszugehen. Der rechte Unterarm wurde im Median mit einem Score von 2 bewertet und lag somit unterhalb der gesundheitlichen Risikoschwelle. Unter Betrachtung der relativen zeitlichen Verteilung fällt auf, dass die Probanden zwischen $20 \%$ und $35 \%$ der gemessenen Arbeitszeit mit einer Haltung arbeiten, die als gesundheitlich riskant eingestuft wurde. Folglich lässt sich feststellen, dass die ergonomische Arbeitsweise der Z̈̈ und ZFAs zwar insgesamt nicht als gesundheitlich riskant einzustufen ist, dass aber eine Tendenz zu riskanten Arbeitshaltungen erkennbar ist. Für den rechten Handgelenk- \& Rotations-Score ist erkennbar, dass dieser im Median mit 4,5 von 6 Punkten bewertet wurde und somit knapp unter der Risikoschwelle ( $\geq 5$ ) liegt. Das dritte 
Quartil liegt allerdings bei einem RULA Score von 5 und in der relativen zeitlichen Verteilung lässt sich erkennen, dass circa 49\% der Arbeitszeit mit einem Score von 5 bewertet wurden, welcher als gesundheitlich riskant eingestuft wird.

Auch die Studiengruppe Park et al.[121] untersuchten anhand von RULA den Einfluss verschiedener zahnmedizinischer Aufgaben auf die ergonomische Arbeitshaltung von ZÄ, welche sie bei der Arbeit filmten. Dabei untersuchten sie den jeweiligen Einfluss auf den RULA Gesamtscore sowie die RULA Teilscores. Für die Analyse wurden die körperlich belastendsten und komfortabelsten Körperhaltungen ausgewählt und bewertet. Für den Oberkörper Teilscore zeigte die Studie RULA Scores zwischen 3 und 5 von maximal 6 Punkten. Damit lag die Bepunktung z.T. deutlich höher als in dieser Studie. Für die Nackenregion beschrieben Park et al. [121] ebenfalls einen RULA Teilscores zwischen 3 und 5 von maximal 6 Punkten, was mit dem medianen RULA Teilscore dieser Studie (3) tendenziell übereinstimmt. Der Oberarm wurde mit einem RULA Teilscore zwischen 1 und 3 Punkten von 6 bewertet und stimmt somit weitgehend mit der Bewertung in dieser Studie (zwischen 1 und 2 Punkten) überein. Der Unterarm wurde von Park et al. [121] mit 2 von 4 Punkten bewertet, was mit dem medianen Teilscore dieser Studie für den Unterarm (zwischen 2 und 2,5) übereinstimmt. Wird als letzte Region der Handgelenk \& Rotationswert betrachtet, wird dieser zwischen 3 und 6 von maximal 6 möglichen Punkten angegeben, was im Mittel mit dem medianen RULA Teilscore dieser Studie $(4,5)$ übereinstimmt. Die in der Vergleichsstudie erhobenen Teilscores bestätigen folglich zumeist die Ergebnisse vorliegenden Studinresultatee. Insgesamt kommt die Vergleichsstudie unter den Probanden je nach Aufgabe zu RULA Gesamtscores zwischen 5 und 7 und bewertet die ergonomische Arbeitsweise insgesamt folglich ähnlich streng wie die vorliegende Studie. Park et al. [121] schlussfolgern, dass die ergonomische Arbeitsweise von ZÄ weitere Untersuchung und Änderung bedarf, da sie ursächlich für hohen physischen Belastungen und einem erhöhtes Risiko für MSE ist. Die Ergebnisse der vorliegenden Studie führen zur gleichen Schlussfolgerung.

In den angeführten Studien [29, 121, 123] finden sich z.T. abweichende Ergebnisse, welche sich auch durch ein abweichendes Studiendesign erklären lassen. So waren die Messbedingungen der einzelnen Probanden in den verglichenen Studien ungleich, da z.B. nicht alle Probanden die gleichen Patienten behandelt und somit nicht die gleiche Aufgabenstellung hatten während ihre ergonomische Arbeitsweise evaluiert wurde. Da die Patien- 
tensituation und mit ihr die an die Behandler gestellte physiologische Anforderung erheblich variieren, lassen sich eingenommenen Körperhaltungen während der Arbeit an verschiedenen Patientenfällen z.T. nur schwer miteinander vergleichen. In der vorliegenden Studie wurden deshalb die gleichen Voraussetzungen für alle geschaffen, um diese Vergleichbarkeit zu gewährleisten. Dafür wurde eine repräsentative Behandlungssimulation am Phantomkopf entworfen, welche alle Probanden in der gleichen Einrichtung und mit dem gleichen Inventar zu bearbeiten hatten. Jeder Proband konnte so den gleichen Patientenfall bearbeiten. In den Vergleichsstudien behandelten die Probanden entweder in den ihnen vertrauten Räumlichkeiten [121] oder die Studien machten keine Angaben [29, 123] zu Räumlichkeiten und Inventar der Messungen. Ein solches Studiendesign verzerrt die Vergleichbarkeit zwischen den einzelnen Probanden, da ein mehr oder weniger ergonomisches Instrumentarium die ergonomische Arbeitsweise des einzelnen Probanden und letztendlich dessen Bewertung beeinflusst.[34-37, 51] Des Weiteren waren die Vergleichsstudien [121-123] weniger komplex und weniger datenintensiv. So wurde die Arbeitsweise von Z̈̈ und ZFAs, z.B. nur videoprotokolliert oder fotografiert. Das Videobzw. Fotomaterial wurde dabei im Nachhinein analysiert und ausgewertet. Z.T. wurden nur besonders hochfrequent eingenommene oder von den Untersuchern als repräsentativ empfundene Arbeitshaltungen zur Bewertung herangezogen. Aufgrund dieser Divergenz der Vergleichsstudien [121-123] ist damit ein Transfer zu den Daten dieser Studie nur eingeschränkt möglich, wodurch auch das Ziehen einheitlicher Schlussfolgerungen limitiert ist. Es bleibt bei den Vergleichsstudien bei einer subjektiven Analyse und Bewertung durch den Untersucher. Diesem ist es u.U. nur möglich einen Gesamteindruck oder besonders einprägsame Arbeitsweisen zu erfassen. Werden z.B. nur häufig eingenommene oder repräsentative Körperhaltungen analysiert und ausgewertet, sind zwar einzelne Arbeitsschritte oder spezifische Risikofaktoren bewertbar, eine ganzheitliche Einschätzung der ergonomischen Arbeitsweise kann aber nicht getroffen werden.[129] Des Weiteren entziehen sich so optisch schwer zu erfassende Bewegungen, wie z.B. die der Handgelenke oder Rumpfrotation z.T. einer präzisen Beurteilung durch den Untersucher und verfälschen so die Bewertung der ergonomischen Arbeitsweise.[129] Im Umkehrschluss ist im direkten Vergleich in der vorliegenden Studie somit von einer präziseren Beurteilung der ergonomischen Arbeitsweise der jeweiligen Probanden auszugehen. Die anhand direkter Messungen gewonnen objektiven Daten lassen keine Verzerrung der Bewertung zu und ermöglichen außerdem die ergonomische Arbeitsweise ganzheitlich und zu jedem 
Zeitpunkt des gesamten Behandlungsverlaufs zu erfassen und zu bewerten. Dennoch besteht Einigkeit darüber, dass die ergonomische Arbeitsweise von Z̈̈ und ZFA weiterer Untersuchung und Änderung bedarf, da sie zumeist als gesundheitsschädlich bzw. gesundheitlich riskant eingestuft ist.

5.2.2 Die ergonomische Risikobeurteilung und die Prävalenz Muskuloskelettaler Erkrankungen von Zahnärzten und Zahnmedizinischen Fachangestellten

Das arbeitsbedingte gesundheitliche Risiko und mit ihr assoziierte gesundheitliche Beeinträchtigung werden in der allgemein hohen Prävalenz von MSE unter ZÄ und ZFAs deutlich.[6, 10, 12, 26, 30] Zwischen der MSE-Prävalenz der Probanden dieser Studie und der MSE-Prävalenz, welche die aktuelle Literatur angibt, findet sich eine große Übereinstimmung. Die Probanden dieser Studie gaben für die letzten 12 Monate an, dass die am öftesten betroffenen Regionen Nacken, Schultern, Oberkörper bzw. Rücken und Handgelenke waren. 86,4\% der Probanden gaben an, in den letzten 12 Monaten unter Beschwerden in der Nackenregion gelitten zu haben. 86,4\% der Probanden hatten Schulterbeschwerden. In der Oberkörperregion gaben die Probanden an, zu 63,6\% Beschwerden im unteren Rücken und zu 31,8\% Beschwerden im oberen Rücken gehabt zu haben. 40,9\% litten an Beschwerden der Handgelenke und Hände. Auch Rafie et al. [29] befragten ihre Probanden zur MSE-Prävalenz der letzten 12 Monate. Die vier meistbetroffenen Körperregionen waren die Nackenregion mit 48,6\% der Probanden, die Schultern mit 42\%, die Oberkörperregion mit 38,8\% und die Handgelenksregion mit 28\%. Statistisch konnten Sie einen signifikanten Zusammenhang $(\mathrm{p} \leq 0,05)$ zwischen der Nacken- und Schulterregion und dem korrespondierenden RULA Score zeigen.

Diese unter den Probanden erfasste Prävalenz muskuloskelettaler Beschwerden stimmt neben der Studie von Rafie et. al [29] auch mit weiterer aktueller Literatur [6, 10, 12, 26, 30] bezüglich Prozentzahlen als auch den am meisten betroffenen Regionen überein. Auch Lietz et al. [6], Ohlendorf et al. [10, 31] und Leggat et al. [26] beschrieben bereits Nacken, Schultern und unterer Rücken als die meistbetroffenen Regionen. Unter tschechischen Zahnärzten gaben 78,1\% an in den letzten 12 Monaten an Nackenbeschwerden gelitten zu haben, 75\% gaben Beschwerden des unteren 49,7\% Beschwerden des oberen Rückens an, 52,2\% hatten Schulterschmerzen und 38,8\% gaben Handgelenksschmerzen an.[30] Ohlendorf et al. [10] beschrieben die 12-Monatsprävalenz für ZFAs. In den letz- 
ten 12-Moanten gaben 85,2\% der Probanden Nackenbeschwerden, 70,2\% Schulterbeschwerden, 60,1\% Beschwerden im unteren und $48 \%$ im oberen Rücken sowie 31,8\% Beschwerden im Handgelenk an. Obwohl muskuloskelettale Beschwerden in den letzten 12 Monaten das Einschlusskriterium für die Teilnahme an dieser Studie war und somit von einer höheren Prävalenz auszugehen wäre, weicht die vorliegende Probandenkohorte in ihrer MSE-Prävalenz nicht bedeutend von den Ergebnissen der anderen Studien ab, sondern bestätigen diese sowohl in den meistbetroffenen Regionen als auch in der Höhe der Prävalenz. Dies betont wie üblich und weitverbreitet MSE unter zahnmedizinischem Personal ist. Außerdem bestärkt die übereinstimmende MSE-Prävalenz unserer Probandenkohorte in ihrem repräsentierenden Charakter für diese Berufsgruppen.

\subsection{Einfluss eines systematischen Krafttrainings auf die ergonomische Arbeitsweise von Zahnärzten und Zahnmedizinischen Fachangestellten}

5.3.1 Ergebnisanalyse und Hypothesenprüfung: Einfluss eines systematischen Krafttrainings auf die Gelenkwinkel und Gelenkpositionen

In diesem Kapitel werden die in Kapitel 4.4 dargestellten Gelenkwinkel und Gelenkpositionen der Prä- und Postmessung auf statistische und deskriptive Unterschiede analysiert, um den Einfluss eines systematischen Krafttrainings zu diskutieren. Dabei werden die in Kapitel 2.9 formulierten Arbeitshypothesen 1a., 1b., 1c. überprüft.

Vor der Diskussion der Ergebnisse, ist darauf hinzuweisen, dass diese Studie in ihrem Aufbau als wissenschaftlich neuwertig zu erachten ist. Die innovative Messtechnik anhand von direkten Messungen und insbesondere den Inertialsensoren erreichte erst in den vergangenen Jahren die notwendige Präzision, um die ergonomische Arbeitsweise adäquat zu untersuchen.[119] Es wurden zwar zahlreiche Arbeitsplätze in unterschiedlichen Berufsspaten mittels dieser Technik überprüft, allerdings nur um einmalig Risikofaktoren des jeweiligen Arbeitsumfelds auszumachen bzw. die jeweilige ergonomische Arbeitsweise zu erfassen.[2, 115, 119, 132, 133, 137, 183] Die Überprüfung des Einflusses eines systematischen Krafttrainings auf die ergonomische Arbeitsweise am selben Arbeitsplatz anhand von objektiven Daten, die durch Inertialsensoren gewonnen wurden, ist in dieser 
Konstellation allerdings ein wissenschaftliches Novum. Die Veränderungen der Gelenkwinkel und Gelenkpositionen sind dabei hilfreich, um unabhängig von speziell zur ergonomischen Risikoevaluation entwickelten Instrumenten die Veränderung der Arbeitshaltung der Probanden unvoreingenommen einzuschätzen.

In der Nackenregion zeigt sich für Extension bzw. Flexion des Nackens, dass während der Prämessung statistisch signifikant $(\mathrm{p} \leq 0,01)$ öfter ein Gelenkwinkel von circa $45^{\circ}$ eingenommen wurde als in der Postmessung. Deskriptiv lässt sich dafür beschreiben, dass während der Postmessung geringfügig öfter höhere Gelenkwinkel $\left(>50^{\circ}\right)$ gemessen wurden. Die Veränderung ist dabei aber auf eine Umverteilung der gemessenen Gelenkwinkel aus dem Bereich um $45^{\circ}$ (Prämessung) in den Bereich $>50^{\circ}$ (Postmessung) zurückzuführen. Es gibt keineklar erkennbare Entwicklungstendenz. Eine klinische Relevanz des signifikanten p-Werts ist somit nicht gegeben. Für die Rotation und Lateralflexion des Nackens zeigen die Ergebnisse keine statistisch signifikante Veränderung beschreibbar und auch deskriptiv sind die beschreibbaren Unterschiede nur marginal. Für die Rotation und Lateralflexion wurden bereits während der Prämessung zum Großteil neutrale Gelenkwinkel gemessen, wodurch eine ausbleibende Veränderung u.U. zu erklären ist. Für die Überprüfung der Arbeitshypothese 1a. lässt sich insgesamt nicht erkennen, dass ein systematisches Krafttraining in der Nackenregion zu mehr Arbeitszeit in neutralen Gelenkwinkeln geführt hat. Somit kann die Arbeitshypothese 1a. falsifiziert werden.

In der Flexion bzw. Extension der Oberkörperregion wurde ein Gelenkwinkel um $15^{\circ}$ statistisch signifikant ( $\mathrm{p} \leq 0,01)$ öfter während der Postmessung verzeichnet. Deskriptiv ist außerdem erkennbar, dass sich der am häufigsten gemessene Gelenkwinkel sich von circa $18^{\circ}$ in der Prämessung auf $10^{\circ}$ in der Postmessung reduziert hat. Die Probanden arbeiteten folglich in neutraleren Gelenkwinkeln während der Postmessung und somit mir aufrechterem Oberkörper. Der signifikante p-Wert ist klinisch somit relevant und lässt sich ggf. dadurch erklären, dass die ausgeprägtere Muskulatur des Oberkörpers den Probanden nach der Trainingsintervention ermöglichte über einen längeren Zeitraum arbeiten zu können ohne dabei der an sie gestellten Arbeitsbelastung nachzugeben zu müssen und sich folglich vorzubeugen. Wird die Rotation des Oberkörpers betrachtet lässt sich weder statistisch noch deskriptiv eine Veränderung erkennen. Für die Lateralflexion konnte ein kleiner Gelenkwinkel um $5^{\circ}$ während der Postmessung statistisch signifikant $(\mathrm{p} \leq 0,05)$ öfter gemessen werden. Deskriptiv zeigen sich insgesamt allerdings nur mini- 
male Varianzen zwischen den beiden Messungen. Eine klinische Relevanz des signifikanten p-Wertes ist folglich nicht gegeben. Für den Oberkörper konnte in 1/3 Gelenkwinkeln gezeigt werden, dass ein systematisches Krafttraining zu mehr Arbeitszeit in neutraleren Gelenkwinkeln geführt hat. Die marginalen Varianzen der anderen beiden Gelenkwinkel lassen sich allerdings dadurch erklären, dass die gemessenen bereits vor der Trainingsintervention fast ausschließlich im neutralen Bereich lagen und dementsprechend nicht noch neutraler werden konnten. Somit kann die Arbeitshypothese 1b. insgesamt bestätigt werden.

Wird nun zuerst die linke Schulter- und Oberarmregion betrachtet, lässt sich für die Flexion bzw. Extension statistisch keine signifikante Veränderung $(\mathrm{p} \geq 0,05)$ beschreiben und auch deskriptiv gibt es nur minimale Varianzen. Für die Elevation der linken Schulter zeigen sich weder statistisch noch deskriptiv relevante Veränderungen. Gleiches gilt für die Abduktion des linken Oberarms. In den beiden letztgenannten Gelenkwinkeln arbeiteten die Probanden bereits während der Prämessung in neutralen bzw. annähernd neutralen Gelenkwinkeln, wodurch sich eine ausbleibende Veränderung erklären lässt. Trotzdem kann insgesamt für die linke Schulter- und Oberarmregion die Arbeitshypothese 1c. nicht bestätigt werden.

Auf der rechten Seite lässt sich für den Oberarm in allen drei gemessenen Gelenkwinkeln statistisch ebenfalls keine signifikante Veränderung ( $\mathrm{p} \geq 0,05)$ erkennen und auch deskriptiv nur wenige Varianzen beschreiben, die keine klare Entwicklungstendenz zeigen. Auch hier arbeiteten die Probanden bereits während der Prämessung bezüglich der Schulterelevation und Abduktion in neutralen Bereichen, wodurch sich eine ausbleibende Veränderung erklären lässt. Auch für die rechte Schulter- und Oberarmregion kann die Arbeitshypothese 1c. nicht bestätigt werden.

Nach der nun abgeschlossenen Hypothesenprüfung für die Gelenkwinkel und Gelenkpositionen können die noch die übrigen Gelenkwinkel für die Unterarmregion sowie die Handgelenks- \& Rotationswerte für beide Körperhälften hinsichtlich des Einflusses eines systematischen Krafttrainings betrachtet werden. Für die linke Unterarmregion ist zwischen Prä- und Postmessung weder für in der Flexion/Extension noch die Gelenkposition des Unterarms eine relevante Veränderung erkennbar. Wird Handgelenk \& Rotation auf der linken Seite betrachtet so ist auch hier keine Veränderung erkennbar und ein Einfluss lässt sich nicht erkennen. Bei Betrachtung der Handgelenksdeviation fällt auf, dass ein Gelenkwinkel im Bereich um $0^{\circ}$ während der Prä-Messung signifikant $(p \leq 0,01)$ häufiger 
eingenommen wird als im Vergleich zur Postmessung. Deskriptiv ist erkennbar, dass während der Postmessung tendenziell weiter von neutralen Gelenkwinkeln abgewichen wurde und sich die entsprechende Mediankurve um ca. $5^{\circ}$ verschoben hat (Abb. XX) Der signifikante p-Wert ist somit klinisch relevant und folglich kann für diese Gelenkwinkel kein Einfluss eines systematischen Krafttrainings hin zu neutraleren Gelenkwinkeln gezeigt werden.

Wird nun die kontralaterale Körperseite betrachtet, lässt sich für den rechten Unterarm beschreiben, dass in der Flexion/Extension ein niedriger Gelenkwinkel um $70^{\circ}$ in der Prämessung signifikant $(\mathrm{p} \leq 0,01)$ häufiger gemessen wurde. Deskriptiv ist eine Verschiebung der gemessenen Gelenkwinkel zwischen Prä- und Postmessung erkennbar, hin zu einer stärkeren Flexion des Unterarms. Für beide Messungen liegen die jeweils am häufigsten gemessenen Gelenkwinkel allerdings im annährend neutralen Bereich und ansonsten sind keine relevanten Varianzen beschreibbar. Der signifikante p-Wert zeigt folglich nur eine eingeschränkte klinische Relevanz und könnte aber als ein beginnender Einfluss eines systematischen Krafttrainings interpretiert werden. Genau beurteilen lässt sich der Einfluss des Krafttrainings folglich nicht. Für die Gelenkposition lässt sich weder statistisch eine signifikante ( $p \geq 0,05)$ noch deskriptiv eine relevante Veränderung beschreiben, während die Probanden in beiden Messungen fast ausschließlich über die Körpermitte bzw. außerhalb der Körpermitte arbeiteten. Folglich ist hier kein Einfluss des systematischen Krafttrainings auf die ergonomische Arbeitsweise erkennbar.

Zusammenfassend lässt sich also sagen, dass nur eine von drei Arbeitshypothesen durch die Ergebnisse bestätigt wurde. Nur in der Oberkörperregion konnte gezeigt werden, dass das systematische Krafttraining in 1/3 Gelenkwinkeln zu mehr Arbeitszeit in neutralen Gelenkwinkeln geführt hat. Nur die Arbeitshypothese 1a. wurde somit verifiziert. Für die Nackenregion sowie die Region von Schultern und Oberarmen wiederum mussten die entsprechenden Arbeitshypothesen 1b. und 1c. falsifiziert werden, da keine Verbesserungen erkennbar waren. Die größtenteils unveränderten Gelenkwinkel suggerieren primär, dass ein systematisches Krafttraining nicht oder nur eingeschränkt zu einer aufrechteren und symmetrischeren Arbeitshaltung führt wie sie für die zahnmedizinischen Berufe gefordert wird.[20-25, 97] Neben der Flexion bzw. Extension des Oberkörpers ist für einige Gelenkwinkel (Flexion/Extension des Nackens, Flexion/Extension Unterarm rechtsseitig) zwar nicht die Aussage machbar, dass das Krafttraining zu mehr Arbeit in neutraleren Gelenkwinkeln geführt hat, es lassen sich aber dennoch zwischen Prä- und Post-Messung 
Varianzen erkennen, die ggf. auf einen einsetzenden posturalen Effekt des Krafttrainings hinweisen könnten. Der Effekt des Krafttrainings auf die Arbeitshaltung wird im folgenden Kapitel 5.3.2 diskutiert.

Aufgrund der Neuartigkeit der verwendeten Technik sowie des hohen betriebenen Aufwands dieser Studie lassen sich die Ergebnisse nur eingeschränkt mit Studien aus der Zahnmedizin oder anderen Berufsgruppen vergleichen. Bisher untersuchte keine Studie die kinematischen Effekte eines systematischen Krafttrainings mittels direkten Messsystemen (z.B. Xsens). Grundsätzlich mangelt es in der wissenschaftlichen Literatur an Untersuchungen, die die posturalen Effekte präventiver Maßnahmen anhand direkter Messungen untersuchen. In der zahnmedizinischen Ergonomieliteratur [4, 101, 106-108] sind zwar verhaltenspräventive Maßnahmen beschrieben, die zu einer Verbesserung der Arbeitshaltung und insbesondere einer Verbesserung der MSE-Prävalenz führen sollen, doch stützen sich die Studien auf subjektive Einschätzungen der Untersucher sowie Eigenangaben von Probanden. Die Beurteilung der Effektivität dieser Maßnahmen beruhte somit nicht auf objektive Daten. Faust et al. [107] untersuchten 41 Zahnmedizinstudenten bezüglich des Einflusses einer 10-minütigen Ergonomieschulung auf die ergonomische Arbeitsweise. Die Untersucher bewerteten in einem Punktesystem während der Behandlung die ergonomische Arbeitsweise des Probanden. Zwischen der Interventionsgruppe und der Vergleichsgruppe beschrieben die Untersucher statistisch signifikante Unterschiede bezüglich des Scores für die ergonomische Position $(p=0,006)$ sowie der Schulterabduktion $(\mathrm{p}=0,03)$ und der Lateralflexion der Wirbelsäule $(\mathrm{p}=0,02)$. Es wurde geschlussfolgert, dass eine solche verhaltenspräventive Maßnahme die ergonomische Compliance und Arbeitsweise von Zahnmedizinern positiv beeinflussen kann. Die Aussagekraft ist dabei aber durch das zugrunde liegende Studiendesign limitiert. Da sich die Untersucher auf ihre subjektive Einschätzung berufen, ist eine ganzheitliche und unvoreingenommene Bewertung nicht möglich.[119, 129] Des Weiteren ist fraglich, ob eine solche ergonomische Intervention den gleichen Effekt auf arbeitserfahrene ZÄ hätte, die bereits seit Jahren an ihre ergonomische Arbeitsweise gewöhnt sind und somit vermutlich schwerer zu beeinflussen wären. Ein Transfer zwischen den subjektiven Daten der Vergleichsstudie und den objektiven Daten dieser Studie ist aufgrund der zahlreichen Unterschiede im Studiendesign nicht möglich. Deghan et al.[108] untersuchten 102 ZÄ bezüglich des Einflusses einer ergonomischen Intervention, welche aus Ergonomieschulungen, 
Modifikationen des Arbeitsplatzes, Ergonomietrainings und einem regelmäßigen Bewegungsprogramm (Stretching-Übungen) bestand, auf die MSE-Prävalenz, die sie anhand eines Fragebogens unter den Probanden erhoben. Sie konnten zeigen, dass eine 8-wöchige ergonomische Intervention die MSE-Prävalenz unter den Probanden signifikant ( $\mathrm{p} \leq$ $0,05)$ verringern konnte und bestätigten somit den positiven Einfluss verhaltenspräventiver Maßnahmen und eines regelmäßigen Bewegungsprogramms. Auch in diesem Fall stützt sich die Studie auf subjektive Daten, die diesmal dem Wohlbefinden der Probanden zugrunde liegen. Dieses wurde von den Untersuchern anhand des Nordic Questionnaire erhoben.[174] Aufgrund der multiplen verhaltenspräventiven Ansätze der Studie lässt sich außerdem kein Rückschluss darüber ziehen, ob ein regelmäßiges Bewegungsprogramm eine adäquate verhaltenspräventive Maßnahme ist. Ob eine einzelne der angewandten Maßnahmen letztendlich zum beschriebenen positiven Effekt geführt hat oder sie nur in ihrer Gesamtheit effektiv sind, bleibt folglich unklar. Der Einfluss der Intervention auf die ergonomische Arbeitsweise wurde wie so oft nicht untersucht und somit lässt sich kein Vergleich zur in dieser Studie untersuchten Effektivität eines systematischen Krafttrainings ziehen.

Neben den wenigen Studien, die verhaltenspräventive Maßnahmen untersuchen, herrschen in der aktuellen zahnmedizinischen Literatur [34, 35, 37, 40, 108] Studien vor, welche den Einfluss verhältnispräventiver Maßnahmen untersuchen. Analysiert wurde unter anderem der Einfluss von Lupenbrillen und OP-Mikroskopen sowie ergonomisch designten Behandlungsstühlen oder ergonomisch designtem Instrumentarium. Fast alle Studien stützten sich wiederum auf subjektive Bewertungen, z.B. anhand observatorischer Methoden und Eigenangaben der Probanden. Dable et al.[34] untersuchten 90 Zahnmedizinstudenten bezüglich des Einflusses von Sattelstühlen auf die ergonomische Arbeitsweise. Diese wurde von den Untersuchern mit RULA bewertet. Die Untersucher beschrieben, dass die Probanden, die mit konventionellen Stühlen arbeiteten, signifikant schlechtere RULA Scores erhielten als mit Sattelstühlen. Der RULA Gesamtscore eines konventionellen Stuhls $(7.01 \pm 0.45)$ war mehr als doppelt so groß wie der eines Sattelstuhls $(2.93 \pm 0.69)$. Die Untersucher kamen zu dem Schluss, dass diese verhaltenspräventiven Maßnahmen helfen, die natürliche Kurvatur des Rückens aufrecht zu erhalten und zu einer aufrechteren Arbeitsweise führen. In der vorliegenden Studie wiederum konnte keine Verbesserung des RULA Gesamtscores und des RULA Oberkörperscores beschrieben werden (siehe Kapitel 5.3.2). Dennoch ließ sich die Arbeitshypothese 1a. 
bestätigen, dass ein systematisches Krafttraining als verhaltenspräventive Maßnahme zu einer insgesamt weniger vorgebeugten Haltung des Rückens bzw. mehr Arbeitszeit in neutralen Gelenkwinkeln geführt hat. Ein Vergleich der RULA Scores und somit Rückschlüsse auf die jeweilige Effektivität der Intervention ist aufgrund der Divergenz zwischen subjektiver und objektiver Bewertung der ergonomischen Arbeitsweise aber nur schwer möglich.

Obwohl die Informationen für eine korrekte und gesunde ergonomische Arbeitsweise in der Zahnmedizin bereits seit vielen Jahren kursieren und Studien, wie oben angeführt, den positiven Einfluss von verhaltens- bzw. verhältnispräventiven Maßnahmen auf die Arbeitshaltung und MSE-Prävalenz bestätigt haben, ist diese Prävalenz in den zahnmedizinischen Berufen nach wie vor sehr hoch.[6, 10, 12, 26, 31] Es wird davon ausgegangen wird, dass die hohe MSE-Prävalenz unter Z̈̈ und ZFAs in der ergonomischen Arbeitsweise und somit auch dem Arbeitsumfeld begründet ist. Dies suggeriert zum einen, dass anhand der bisherigen Studien und ihrer Untersuchungsmethoden die der hohen MSEPrävalenz zugrundeliegenden ergonomischen Risiken und biomechanischen Belastungen nicht ausreichend ergründet werden konnten. Zum anderen stellte sich die Frage, inwiefern bisher untersuchte und implementierte verhaltenspräventive und verhältnispräventive Maßnahmen, trotz ihrer in Studien nachgewiesenen Effektivität, tatsächlich Einfluss auf die ergonomische Arbeitsweise nehmen können. Es stellt sich außerdem die Frage, ob verhaltens- und verhältnispräventive Maßnahmen überhaupt an der richtigen Stelle ansetzen. Wie bereits mehrfach erwähnt wurde gibt es bisher in der zahnmedizinischen Literatur nur wenige Studien, die ergonomische Arbeitsweise unter ZÄ und ZFAs anhand objektiver Daten untersucht haben.[11, 37, 51, 183] Suedbeck et al.[37] untersuchten den Einfluss ergonomisch designter Instrumente auf die Muskelaktivität des Unterarms in einer Behandlungssimulation am Phantomkopf. 27 ZFAs wurden während Scalingübungen mit unterschiedlich designten Küretten mittels einer Oberflächenelektromyographie untersucht. Die objektiven Ergebnisse konnten zeigen, dass schwerere Instrumente eine signifikant höhere Muskelaktivität erforderten. In Übereinstimmung gaben die Probanden der Studie an, dass sie Instrumente mit weniger Gewicht präferierten. Auf Grundlage dieser Daten schlussfolgerten die Untersucher, dass ZFAs ihre Arbeitsbelastung verringern und ergonomische Arbeitsweise verbessern können, wenn sie leichtere Instrumente mit einem größeren Durchmesser verwenden. Wie in der vorliegenden Studie wurde für alle Probanden gleiche Messbedingungen geschaffen, um die Vergleichbarkeit der gewonnen 
Daten zu gewährleisten. Die Arbeit mit objektiven Daten erlaubte den Untersuchern dabei die Effektivität des Instrumentendesigns unvoreingenommen und präzise zu beurteilen.Dadurch konnte die Effektivität dieser verhältnispräventiver Maßnahme von den Untersuchern bestätigt werden. Die subjektive Bestätigung dieser Daten durch die Präferenz der Probanden unterstreicht dabei den Wert der gewonnenen Informationen.[37] Hauck et al.[183] untersuchten anhand des direkten Messsystems CUELA 21 Kieferorthopäden bei ihrer alltäglichen Arbeit bezüglich ihrer ergonomischen Arbeitsweise und bewerteten diese anhand von OWAS. Die gewonnenen kinematischen Daten zeigten, dass die Probanden während der Patientenbehandlung bezüglich der Haltung von Kopf und Wirbelsäule schlechter bewertet wurde als während anderer Aktivitäten. Während der Behandlung nahmen die Probanden 23,4\% der Zeit eine rotierte oder gebeugte Haltung ein. Auch hier griffen die Untersucher auf ein direktes Messsystem zurück, um die ergonomische Arbeitsweise ihrer Probanden präzise, unverfälscht und in ihrer Gesamtheit zu erfassen. Die gewonnen objektiven Daten ermöglichte es den Untersuchern, die Tätigkeiten im Tagesgeschäft von Kieferorthopäden zu isolieren, welche ein hohes ergonomisches Risiko haben. Sie konnten außerdem wie die vorliegende Studie anhand objektiver Daten zeigen, wie oft ein ergonomisches Risiko besteht, wenn die Probanden in einer vorgebeugten und rotierten Haltung arbeiteten.[183] Die angeführten Beispiele zeigen, dass objektive Daten ein präziseres Erfassen vom Arbeitsumfeld bzw. der ergonomischen Arbeitsweise von ZÄ und ZFA ermöglichen als subjektive Daten es können. Aus der unverändert hohen MSE-Prävalenz erschließt sich folglich die Forderung einer neuen, präziseren Einschätzung des Berufsrisikos und der Herkunft von MSE anhand objektiver Daten und ebenso einer Überprüfung der Effektivität ihr zugrunde liegender verhaltens- und verhältnispräventive Maßnahmen.

Die unverändert hohe MSE-Prävalenz $[6,10,12,31]$ trotz der beschriebenen Interventionsmöglichkeiten suggeriert außerdem, dass sowohl verhältnispräventive als auch verhaltenspräventive Maßnahmen u.U. nur einen eingeschränkten Einfluss auf die ergonomische Arbeitsweise in zahnmedizinischen Berufen haben. Das hieße im Umkehrschluss, dass das Arbeitsumfeld und insbesondere der Patient und sein Mund die ergonomische Arbeitsweise in gewissem Ausmaße diktieren. Die Behandler sind somit in ihrer Haltung regelrecht gefangen und haben nur eingeschränkt die Möglichkeit Einfluss zu nehmen. $\mathrm{Zu}$ einem ähnlichen Schluss kam bereits die Arbeitsgruppe von Finsen et al.[7]. Diese untersuchte 107 Z̈̈ bezüglich ergonomischer Risikofaktoren, die zur Entwicklung von 
MSE beitragen. Anhand eines Fragebogens wurden die Arbeitsbedingungen/-gewohnheiten sowie die MSE-Prävalenz der Probanden erhoben. Die drei meistregistrierten Arbeitsaufgaben wurden von 8 Probanden durchgeführt, welche dabei auf ihre ergonomische Arbeitsweise und ihre Arbeitsbelastung anhand einer Elektromyographie untersucht wurden. Die Untersucher registrierten eine hohe statische Muskelaktivität des Musculus Trapezius und Musculus Splenius sowie prolongierte Einnahme von gebeugten Haltungen des Nackens und abduziertem Oberarm während der Behandlung. Die Untersucher konnten diese Arbeitsweise mit einer permanent hohen Präsenz ergonomischer Risikofaktoren im Arbeitsumfeld der Probanden in Verbindung bringen. Finsen et al.[7] schlussfolgerten, dass Arbeitsabläufe in der Zahnmedizin kaum anzupassen sind, da sie anatomisch durch die Mundhöhle vorgegeben werden und ergonomische Interventionen somit stark limitiert sind. Das bereits diskutierte Berufsbild von Z̈̈ und ZFAs macht es notwendig, dass die Behandler ihre Arbeitsweise an die individuellen Gegebenheiten des Patienten anpassen. Die oftmals während der Patientenbehandlungen eingenommenen Zwangshaltung mit statischer Muskelkontraktion, wie Finsen et al.[7] und andere Studien [3, 4, 6, 12, 28, $66,73]$ sie beschreiben, lassen sich mit diesem Bild sehr gut in Einklang bringen. Die hohen MSE-Prävalenzen sowie die Ergebnisse dieser Studie suggerieren, dass Z̈̈ und ZFAs während der Behandlung oftmals von neutralen, aufrechten und symmetrischen Körperhaltungen abweichen und dass auch aktuell implementierte verhaltens- und verhältnispräventive Maßnahmen (z.B. Ergonomieschulungen/-Training, Strechtchingübungen, Sattelstühle, Lupenbrillen/OP-Mikroskope, Instrumentendesign) [34, 35, 37, 90, 108] dies nicht ausreichend verhindern können. Für ein tieferes Verständnis wie sich ergonomische Arbeitsweisen effektiv beeinflussen lassen, sind zukünftige Untersuchungen anhand objektiver Daten bzw. direkter Messungen zwingend. Die vorliegende Studie konnte somit insgesamt nur einen geringen bzw. eingeschränkten Einfluss eines 10- bis 12-wöchigen Krafttrainings auf die ergonomische Arbeitsweise zeigen. Von der Arbeitsweise unabhängig konnten aber dennoch bereits einige Studien[8, 39, 40, 46, 47, 49, 165] den positive Einfluss von körperlichem Training auf das subjektive Wohlbefinden sowie das Auftreten muskuloskelettaler Beschwerden zeigen. Dieser Einfluss wird im folgenden Kapitel 5.3.2 diskutiert. 
5.3.2 Ergebnisanalyse und Hypothesenprüfung: Einfluss eines systematischen Krafttrainings auf die ergonomische Risikobeurteilung

In diesem Kapitel wird die in Kapitel 4.1 und 4.2 dargestellte ergonomische Risikobeurteilung bzw. die relative zeitliche Verteilung gesundheitlich riskanter Bewertungsparameter der Prä- und Postmessung auf deskriptive und statistische Unterschiede analysiert, um den Einfluss eines systematischen Krafttrainings zu diskutieren. Da beide Auswertungsparameter aufeinander aufbauen und gemeinsam auf der Bewertung durch den RULA Arbeitsbogen[120] fußen, werden beide in diesem Kapitel für die jeweiligen Regionen gemeinsam diskutiert. Dabei werden die in Kapitel 2.9 formulierten Arbeitshypothesen 2a., 2b., 2c. sowie 3a., 3b., 3c. überprüft.

Werden zuerst die RULA Gesamtscores der vorliegenden Studie betrachtet so ist weder statistisch $(\mathrm{p} \geq 0,05)$ noch deskriptiv eine Veränderung erkennbar. Der mediane Gesamtscore und auch das obere wie untere Quartil liegen bei Prä- sowie Postmessung beim Höchstwert 7. Wird die relative zeitliche Verteilung der Bewertungsparameter betrachtet so kann statistisch keine signifikante $(\mathrm{p} \geq 0,05)$ Veränderung beschrieben werden. Deskriptiv zeigt sich, dass die Probanden vor der Trainingsintervention 75,88\% und nach der Trainingsintervention 81,30\% im als gesundheitlich riskant eingestuften Score 7 verbrachten. Somit arbeiteten sie während der Postmessung nicht weniger in einer insgesamt als gesundheitlich riskant bewerteten Arbeitshaltung.

Werden als nächstes die Arbeitshypothesen der ergonomischen Risikobeurteilung 2a., 2b., 2c. sowie 3a., 3b., 3c überprüft lässt sich vorwegnehmen, dass von allen Parametern nur die relative zeitliche Verteilung der Risikoscores des Nackens eine statistisch signifikante $(\mathrm{p} \leq 0,05)$ Veränderung zeigt. Die anderen Parameter zeigen dennoch z.T. deskriptive Varianzen. Für den RULA Nackenscore lässt sich deskriptiv eine Veränderung des oberen Quartils beschreiben, welches sich von einem Wert von 4, der gesundheitlich riskant eingestuft ist, auf einen Wert von 3,5, der nicht als gesundheitlich riskant eingestuft ist, reduziert. Die Arbeitshypothese 2a. lässt sich somit bestätigen, da ein systematischen Krafttraining zu einer besseren objektiven Bewertung der Nackenregion geführt hat. Die zeitliche Verteilung der riskanten RULA Bewertungsparameter reduzierte sich statistisch signifikant $(\mathrm{p} \leq 0,05)$ zwischen Prä- und Postmessung. Da sich der prozentuelle Anteil, den die Probanden in riskanten Bewertungsparametern verbrachten, nur um 3,72\% von $44,10 \%$ auf $40,38 \%$ verringerte lässt sich allerdings nur von einer geringen klinischen 
Relevanz sprechen. Trotzdem lässt sich auch die Arbeitshypothese 3a., dass ein systematisches Krafttraining objektiv zu weniger Arbeitszeit in gesundheitlich Riskanten ergonomischen Arbeitsweisen führt, für die Nackenregion bestätigen. Da sich der RULA Teilscore der Nackenregion aus der Bepunktung der Flexion/Extension, Rotation und Lateraflexion zusammengesetzt, ist eine verbesserte Bewertung u.U. damit zu erklären, dass das systematische Krafttraining bei einigen Probanden die stützende und haltende Muskulatur dieser Region in solchem Maße gestärkt wurde, sodass diese während der Postmessung länger in ergonomisch adäquaten Haltung arbeiten konnten, ohne zu ermüden und folglich weniger in gebeugte/rotierte Haltungen verfielen. Die geringeren gemessenen Gradzahlen dieser Probanden führten somit zu einer niedrigeren Bepunktung.

Wird der Oberkörper betrachtet, lässt sich deskriptiv nur eine Veränderung des oberen Quartils beschreiben, welches von einem Wert von 2,5 vor der Trainingsintervention sich auf einen Wert von 3 geringfügig erhöht. Die Arbeitshypothese 2b. kann somit falsifiziert werden. Die relative zeitliche Verteilung der gesundheitlich als riskant eingestuften Parameter verändert sich nur marginal von 2,47\% während der Prämessung auf 1,95\% während der Postmessung. Die Arbeitshypothese 3b., dass ein systematisches Krafttraining objektiv zu weniger Arbeitszeit in gesundheitlich Riskanten ergonomischen Arbeitsweisen führt, lässt sich somit zwar verifizieren, hat aber aufgrund des geringen Unterschieds nur eine eingeschränkte Aussagekraft. Die ausbleibende bzw. marginale Veränderung lässt sich u.U. damit erklären, dass bereits während der Prämessung die ergonomische Arbeitsweise der Probanden so gut wie nicht als gesundheitlich riskant eingestuft wurde und das systematische Krafttraining so keinen großen Effekt auf die Bewertung erzielen konnte.

In der linken Schulter- und Oberarmregion erhöhen sich sowohl der Medianwert als auch das obere Quartil geringfügig. Die relative zeitliche Verteilung der Risikoscores verändert sich nicht; sie lag sowohl vor als auch noch dem Interventionszeitraum bei 0\%. Somit kann die Arbeitshypothese 2c. abgelehnt werden, da ein systematisches Krafttraining bei den Probanden dieser Studie nicht zu einer verbesserten Bewertung der ergonomischen Arbeitsweise geführt hat. Die Arbeitshypothese 3c. kann ebenfalls falsifiziert werden, da die Arbeitsweise der Probanden aber bei beiden Messungen zu jeweils keinem Zeitpunkt als gesundheitlich riskant eingeschätzt wurde ist die Aussagekraft der Falsifikation folglich eingeschränkt. Die ausbleibende Veränderung lässt sich ggf. damit erklären, dass die 
Bewertung der ergonomischen Arbeitsweise bereits bei der Prämessung sehr niedrig ausfiel und als nicht gesundheitlich riskant eingestuft wurde. Somit waren keine deutlichen Verbesserungen möglich.

Für die rechte Körperregion der Schultern und Oberarme lässt sich weder statistisch noch deskriptiv eine Veränderung erkennen. Ähnlich wie links zeigt auch auf der rechten Seite die relative Zeitliche Verteilung der als gesundheitlich riskant eingestuften Bewertungsparameter keine Veränderung, da sie jeweils bei 0\% liegt. Somit lassen sich auch hier die Arbeitshypothesen 2c. und 3c. nicht bestätigen. Die Erklärung der ausbleibenden Veränderung ist äquivalent der für die linke Schulter- und Oberarmregion.

Für die Unterarmregion der linken Seite hat sich bei der Bewertung der ergonomischen Arbeitsweise lediglich der Medianwert der Bewertungen geringfügig erhöht. Auch die relative zeitliche Verteilung zeigt, dass die Probanden nach der Trainingsintervention mehr Zeit in gesundheitlich riskant Parametern arbeiteten. Es kommt folglich zu keiner Verbesserung der objektiven Bewertung der ergonomischen Arbeitsweise. Für die linke Handgelenks- und Rotationsregion bleiben Medianwert und oberes Quartil der Bewertungen unverändert bei gesundheitlich riskant eingestuften Werten. Die relative zeitliche Verteilung zeigt, dass der prozentuelle Anteil der in riskanten Scores verbrachten Arbeitszeit von Prä- zu Postmessung zunahm. Als einzige Veränderung lässt sich beschreiben, dass das untere Quartil der Bewertungen sich während der Postmessung geringfügig in Richtung einer niedrigeren Bewertung vorschoben hat. Insgesamt kann aber nicht davon gesprochen werden, dass das Training einen positiven Einfluss auf die ergonomische Arbeitsweise der Probanden ausgeübt hat. Eine ausbleibende Veränderung lässt sich dadurch erklären, dass das systematische Krafttraining primär auf die Körperregionen (Nacken, Rücken, Schultern/Oberarme) mit den höchsten MSE-Prävalenzen unter ZÄ und ZFAs ausgerichtet war. Übungen wie das horizontale Rudern und „Wandwandern“ beanspruchten zwar auch die Unterarmmuskulatur, grundsätzlich war das systematische Krafttraining aber nicht explizit auf die Stärkung dieser Region ausgerichtet. Um Aussagen über den Effekt eines systematischen Krafttrainings auf die Unterarm- und Handgelenksregion zu machen, müssten also ggf. spezifischere Übungen durchgeführt werden. Auf der anderen Körperseite verändert sich die Bewertung des rechten Unterarms nur im dritten Quartil geringfügig zu einer höheren Bewertung. Die relative zeitliche Verteilung zeigt, dass die Probanden während der Postmessung deutlich mehr in gesundheitlich ris- 
kanten Scores arbeiteten als während der Prämessung. Es lässt sich folglich keine verbesserte Bewertung der ergonomischen Arbeitsweise feststellen. Für das rechte Handgelenk ist die Bewertung der ergonomischen Arbeitsweise unverändert auf der definierten gesundheitlichen Risikoschwelle. Die relative zeitliche Verteilung der Bewertungsparameter zeigt, dass während der Postmessung die Arbeitsweise der Probanden nicht seltener mit gesundheitlich riskanten Scores bewertet wurde als während der Prämessung. Das systematische Krafttraining hat demnach auch hier zu keiner Verbesserung der Bewertung der ergonomischen Arbeitsweise geführt.

Zusammenfassend kann folglich nur für die Nackenregion und in geringem Maße für die Oberkörperregion die gestellten Arbeitshypothesen 2a., 3a und 3b. verfiziert werden. Für alle anderen Regionen kann bei den jeweiligen Parametern die Arbeitshypothese nicht bestätigt werden bzw. wurden abgelehnt, weil entweder keine Veränderung oder keine Verbesserung erkennbar ist. Arbeitshypothesen 2b., 2c. und 3c können somit falsifiziert werden.

Grundsätzlich ist darauf hinzuweisen, dass der Einfluss von körperlicher Aktivität auf die ergonomische Arbeitsweise und ergonomische Risikobeurteilung bisher nur unzureichend erforscht ist, sowohl in der Zahnmedizin als auch die Arbeitshaltung und -belastung ihr verwandten Berufe. Vergleichbare Interventionsstudien, die den Einfluss von körperlichem Training untersuchten, taten dies fast ausschließlich anhand von Eigenangaben der Patienten bezüglich der Prävalenz ihrer muskuloskelettalen Beschwerden.[4649, 52, 53, 165] Einzig eine australische Pilotstudie [50] untersuchte bisher den Einfluss eines 10-wöchigen körperlichen Trainings auf die Arbeitshaltung von 23 Zahnmedizinstudenten, welche vor und nach der Trainingsintervention mit RULA bewertet wurde. Auch diese Studie unterscheidet sich aber grundlegend in ihrem Aufbau, wodurch nur eine relative Vergleichbarkeit zur vorliegenden Studie gegeben ist. Das durchgeführte Training wurde zwar ebenfalls als Krafttraining („Resistance Training“) beschrieben, in den Übungen der Vergleichsstudie trainierten die Probanden aber ausschließlich gegen den Widerstand von Thera-Bändern. Das Training beinhaltete 4 Übungen à 2 Minuten für Nacken, oberen Rücken und Schultern, die 5-mal die Woche durchgeführt werden sollten. Die Probanden wurden angewiesen jede Übung bis zur Ermüdung durchzuführen. Bezüglich einiger Belastungsnormativa, wie z.B. Belastungsdauer und Belastungsintensität, machte die Studie allerdings keine präzisen Angaben. Die Belastungsintensität sollte für eine ausreichend hohe Muskelspannung z.B. zwischen $60 \%$ bis $90 \%$ des 1RM liegen, da 
geringere Intensitäten nicht als adäquater Reiz für den Kraft- und Muskelzuwachs angesehen werden.[153-155] Die Belastungsdauer und der Belastungsumfang sollte nach den Angaben der Literatur [42, 159, 160] zwischen 6 bis 15 Wiederholung einer Übung pro Satz liegen, wobei jeweils 3 bis 6 Sätze durchgeführt werden sollen. Die Überlegenheit eines Mehrsatztraining wurde dabei wissenschaftlich belegt. Außerdem scheinen hohe Belastungsumfänge erforderlich, um Veränderungen des passiven Bewegungsapparats zu erzielen.[161] Da weder über die Belastungsintensität noch über die genaue Belastungsdauer genauere Angaben gemacht wurden, ist fraglich, ob eine ausreichender Belastungsreiz erzielt wurde und ob die Anzahl der durchgeführten Wiederholungen einem Krafttraining entsprechend war. Beim geringen Belastungsumfang erscheint es außerdem fraglich, ob das Training einen ausreichenden Einfluss auf den passiven Stütz- und Bewegungsapparat ausüben konnte. In der vorliegenden Studie wurde zu Trainingsbeginn die adäquate 1RM an den dafür ausgerüsteten Trainingsgeräten anhand einer Eingangsmessung objektiv bestimmt. Die Übungen wurden außerdem auf 12 Wiederholungen pro Satz à 3 Sätze ausgelegt. Belastungsumfang und -intensität wurden adäquat gewählt für einen gezielte Muskelaufbau, da eine ausgeprägte Muskulatur als Stützkorsett bei jeglicher körperlichen Aktivität dient und aus präventiver Sicht zudem zur Entlastung von Gelenken sowie der Minimierung der Verletzungsgefahr des Halte- und Stützapparats führt.[42, 43] Die Qualität und Quantität der durchgeführten Übungen ist des Weiteren zu hinterfragen, da die Dokumentation sowie Ausführung den Probanden größtenteils selbst überlassen war und die Probanden der australischen Studie nur bei jeder fünften Trainingseinheit von einem Trainer betreut wurden. Falsch ausgeführte Übungen sowie fälschliche Angaben der Probanden bezüglich ihres Trainings oder ihrer Trainingsbeteiligung könnten so die Ergebnisse der Studie verfälscht haben, da sie sich größtenteils der Kontrolle der Untersucher entzogen. Durch die Betreuung jeder Trainingseinheit in Kleingruppen konnte in der vorliegenden Studie zum einen für alle Probanden gleiche Trainingsbedingungen und eine gleichmäßig hohe Trainingsbeteiligung geschaffen werden. Zum anderen konnte eine hohe Trainingsqualität, z.B. im Sinne einer korrekten und sauberen Ausführung der Übungen, aber auch einer adäquaten Belastungssteigerung über den gesamten Trainingszeitraum durch exaktes Protokollieren und die Expertise der qualifizierten Trainer aufrecht erhalten werden. In einer systematischen Übersichtsarbeit untersuchten Coury et al. [172] 18 Studien, welche die Effektivität körperlichen Trainings zur Kontrolle von Nacken-, Schulter- und Rückenbeschwerden beschrieben. Von 12 Studien mit Betreuung 
der Probanden zeigten 8 Studien einen positiven Effekt bezüglich der Kontrolle von Beschwerden, 2 der Studien wurden als qualitativ hochwertig bewertet. Von 5 Studien ohne Betreuung wiederum zeigten 4 Studien keinen Effekt. Ein Training ohne Betreuung wurde von den Untersuchern folglich als ineffektiv eingeschätzt.

Die Vergleichsstudie von Yiu et al.[50] ist innovativ, da sie als erste den Einfluss eines körperlichen Trainings auf die Körperhaltung und das ergonomische Risiko anhand von RULA untersucht. Die Resultate der Auswertung mit RULA werden im Folgenden mit den Ergebnissen dieser Studie verglichen. Da die ergonomische Beurteilung allerdings auch hier anhand der subjektiven Einschätzung der Untersucher stattfand und nicht anhand objektiver Daten, ist eine Aufschlüsselung der Ergebnisse in die relative zeitliche Verteilung der Bewertungsparameter nicht gegeben, ebenso wie die Aufschlüsselung der Bewertung in Teilscores der einzelnen Regionen. Somit lassen sich nur die RULA Gesamtscores vergleichen. Yiu et al.[50] konnten zeigen, dass in ihrer Studie der mittlere RULA Gesamtscore sich statistisch signifikant $(\mathrm{p} \leq 0,01)$ reduzierte. Nach dem 10-wöchigen Interventionszeitraum sinkt der Präscore von 6 auf einen Postscore von 4,3. Hier konnte die Bewertung der ergonomischen Arbeitsweise folglich deutlich verbessert werden und es wäre nach dem Training von einer aufrechteren, entspannteren Arbeitshaltung auszugehen, die gekennzeichnet ist durch weniger statische Arbeitsweisen und geringere Arbeitslasten. Die Ergebnisse der subjektiven Daten der Vergleichsstudie widersprechen somit den Ergebnissen der vorliegenden Studie Studie. Die Einschränkungen von Daten, die auf subjektiver Einschätzung der Untersucher beruhen, wurden bereits hinreichend in vorangegangenen Kapiteln diskutiert. Es ist somit möglich, dass die beschränkte Wahrnehmung und eine Mögliche Voreingenommenheit der Untersucher $\mathrm{zu}$ niedrigeren RULA-Scores geführt hat. Da das Probandenkollektiv, im Gegensatz zur vorliegenden Studie, außerdem aus Zahnmedizinstudenten und nicht aus zum praktizierenden Z $\ddot{A}$ und ZFAs bestand, sondern aus Zahnmedizinstudenten, könnte eine verbesserte Arbeitshaltung somit genauso zurückzuführen sein auf eine fortgeschrittene Gewöhnung an das Arbeitsumfeld und eine gewachsene Arbeitserfahrung. Da die Studenten gerade erst vertraut werden mit ihrem Arbeitsumfeld, dem Instrumentarium und dem Umgang mit Patienten machen 10 Wochen ggf. einen deutlichen Unterschied bezüglich ihrer Arbeitsroutine. Die Probanden waren so bei der Postmessung u.U. weniger angespannt und hatten eine bessere propriozeptive Wahrnehmung bezüglich ihrer Ergonomie als beim ersten Mal. Eine Kontrollgruppe, die dies ausschließt, fehlt. 
Obwohl das Trainingsprogramm der vorliegenden Studie exakt nach den wissenschaftlichen Vorgaben [42, 43, 144-146, 154] eines systematischen Krafttrainings bzw. eines Hypertrophietrainings aufgebaut war und alle Probanden das Trainingsprogramm strikt einhielten, was von den Trainern und Untersuchern genauestens protokolliert wurde, konnte am Ende des Trainingszeitraums nur ein geringer bzw. eingeschränkter Einfluss auf die ergonomische Arbeitsweise nachgewiesen werden. In der Zahnmedizin in Arbeitsbelastung und Arbeitshaltung verwandten Berufe wurde der positive Effekt eines systematischen Krafttrainings zwar bereits vielfach beschrieben, allerdings untersuchten diese Studien den Einfluss auf die MSE-Prävalenz der jeweiligen Probanden und nicht auf ihre ergonomische Arbeitsweise.[44, 46, 47, 49, 165] Andersen et al. [165] untersuchte z.B. den Einfluss eines spezifischen Krafttrainings, welches direkt die betroffenen Muskelgruppen trainiert, anhand von Probanden mit chronischen Nackenschmerzen (diagnostiziert mit Trapeziusmyalgie). Nach 10-wöchigem Training konnte dabei zu einer signifikanten Reduktion der Schmerzintensität um 35mm auf einer Visuellen AnalogSkala von 0 bis $100 \mathrm{~mm}(\mathrm{p}<0,001)$ beschrieben werden. Spezifisches Krafttraining führte zu prolongierter Schmerzlinderung im Nacken. Eine dänische Studie [48] untersuchte, unter 40 Laborarbeitern, den Einfluss eines Ganzkörperkrafttrainings am Arbeitsplatz. Die Probanden führten dabei drei Mal die Woche über 8 Wochen ein 20-minütiges Training mit Kettelbelts durch. In der Trainingsgruppe konnte dabei verglichen mit einer Kontrollgruppe auf einem Schmerz-Intensität-Index von 1 bis 10 die Schmerzintensität im Nacken- und Schulterbereich um 2,1 Punkte und im unteren Rücken um 1,4 Punkte gesenkt werden. Die schmerz- und beschwerdelindernden Effekte von Krafttraining werden dabei z.B. einer verstärkten Freisetzung von endogenen Opioiden durch angeregte Spannungsrezeptoren der Muskulatur zugeschrieben.[171, 172] Außerdem wird beschrieben, dass Krafttraining zu einem Wachstum der Blutkapillaren und somit einer besseren Versorgung der Muskulatur und Beseitigung von Stoffwechselrückständen führt. Viele weitere Zusammenhänge in der Beziehung zwischen Krafttraining und einer reduzierten MSE-Prävalenz wurden bereits untersucht.[171, 172] Im Gegensatz dazu bleibt die Beziehung zwischen körperlichem Training und der Körperhaltung weitgehend unbekannt.[50] Die Ergebnisse der vorliegenden Studie suggerieren, dass ein 10 bis 12 wöchiges systematisches Krafttraining die Körperhaltung von Z̈̈ und ZFAs während der Arbeit nur in einem sehr eingeschränkten Ausmaß beeinflussen kann. Dies lässt sich ver- 
mutlich damit erklären, dass die ergonomische Arbeitsweise während der Patientenbehandlung auf ein koordinatives Muster zurückzuführen ist, dass die Probanden sich über viele Arbeitsjahre angewöhnt haben. Da im Rahmen der Studie die Probanden keine neuen Techniken zur ergonomischen Arbeit am Patienten erlernt haben und die ergonomische Arbeitsweise nicht korrigiert wurde, ist es naheliegend, dass die Probanden trotz einer ausgeprägteren Muskulatur weiterhin auf die gewohnte Art und Weise arbeiteten. Unter Umständen ist der z.T. ausbleibende Effekt aber auch auf eine zu kurze Dauer der Trainingsintervention zurückzuführen. Es konnte festgestellt werden, dass in den ersten 8 bis 12 Wochen eines Krafttrainings primär neuronale Adaptionsmechanismen (interund intramuskuläre Koordination) in der betroffenen Muskulatur zu einem Maximalkraftzuwachs führen, ohne dass es $\mathrm{zu}$ vergleichbaren Zunahmen von Muskelmasse kommt.[42] Morphologische Adaptionsmechanismen im Zuge eines Hypertrophieeffekts der Muskulatur führen erst nach mehreren Monaten des Trainings zum entscheidenden Faktor für Maximalkraftzuwachs.[184, 185] Es kann also davon ausgegangen werden, dass es unter den Probanden dieser Studie zu einer verbesserten inter- und intramuskuläre Koordination und in Folge dessen einer erhöhten Maximalkraft gekommen ist. Ob sich ein Hypertrophieeffekt der Muskulatur eingestellt hat, lässt sich aber nicht abschließend sagen, da die Muskelquerschnitte der Probanden nicht gemessen wurden. Unter Umständen könnten aber gerade diese morphologischen Adaptionsmechanismen einen Einfluss auf die Körperhaltung und somit auch die ergonomische Arbeitsweise ausüben. Um diese und viele weitere Fragen bezüglich des Zusammenhangs von körperlichem Training und der Körper- bzw. Arbeitshaltung zu klären, sind in Zukunft weitere Untersuchungen mit u.U. längeren Interventionszeiträumen nötig.

Die Krafttrainingsstudien [46-49, 52, 165] mit anderen Berufsgruppen bestätigen dennoch, so wie es in der Sportwissenschaft beschrieben wird, dass eine gut ausgeprägte Muskulatur zu einem Muskelkorsett führt, welches die auf Halte- und Stützapparat einwirkende Last besser tragen und tolerieren kann und folglich weniger Beschwerden auftreten. [42] Für die vorliegenden Studienergebnisse heißt dies im Umkehrschluss, dass obwohl die objektive Beurteilung der ergonomischen Arbeitsweise von ZÄ und ZFA nur teilweise eine Verbesserung zeigt, ein systematisches Krafttraining dennoch einen subjektiven Einfluss auf die die muskuloskelettalen Beschwerden und das individuelle Wohlbefinden haben kann. Wie es die zahnmedizinische und die Literatur anderer Berufsgruppen $[46-49,52,165]$ suggeriert, ist es folglich wahrscheinlich, dass ein systematisches 
Krafttraining die MSE-Prävalenz unter Z ̈̈ und ZFAs senken kann, auch wenn die ergonomische Arbeitshaltung selbst sich nur wenig oder nicht verändert. Der Zusammenhang, dass eine verbesserte physische Fitness und einen positiven Effekt auf die Prävalenz von MSE hat, wurde außerdem bereits mehrfach in umfragebasierten Studien unter Z ̈̈ und ZFAs bestätigt.[8, 39, 40, 138] Die angeführten Studien zeigten, dass Probanden, die regelmäßig ein körperliches Training durchführten, weniger muskuloskelettale Beschwerden angaben, als ihre untätigen Kollegen. Talpos-Niculescu et al. [8] schlussfolgerten, dass eine erhöhte muskuläre Belastbarkeit zu einer größeren Toleranz gegenüber den extremen körperlichen Anforderungen von dentalen Professionen führt und damit ein effektives Mittel ist, um MSE präventiv entgegen zu wirken und existierende Beschwerden zu minimieren. Lehto et al. [39] beschrieben als mögliche muskelphysiologische Zusammenhänge dieses Effekts eine verbesserte Versorgung der Muskulatur mit Sauerstoff und anderen Nährstoffen, welche z.B. das Auftreten von Hypoxie verhindert. Außerdem wurde ein positiver Einfluss einer verbesserten funktionellen Motorik auf nicht-muskuläre Elemente des Bewegungsapparats, wie z.B. Gelenke und Bänder, als mögliche Ursache diskutiert. Der genaue Zusammenhang zwischen der Wirkung von körperlichem Training und seinem Effekt auf die MSE-Prävalenz in der Zahnmedizin bleibt allerdings unklar.

Um diesen Zusammenhang zu erklären, fehlen in der zahnmedizinischen Literatur experimentelle Vergleichsdaten bezüglich des Einflusses körperlicher Aktivität bzw. körperlichen Trainings. Die wenige verfügbare Literatur [38, 53] werteten andere Parameter als die vorliegende Studie aus, konnten aber denn positiven Einfluss von körperlichen Training bei zahnmedizinischem Personal zeigen. Eine schwedische, randomisierte Kontrollstudie [53] untersuchte 62 Probanden auf die Effekte eines Trainings mittlerer bis hoher Intensität zwei Mal wöchentlich für ca. eine Stunde während der Arbeitszeit. Die Ergebnisse zeigten, dass die subjektiven Beschwerden der Oberkörperregion der Probanden sowie deren Blutglukosespiegel gesenkt werden konnten. Die Art des Trainings wurde dabei allerdings den Probanden überlassen und welches Training die Probanden letztendlich durchführten, wurde nicht in die Studie mit aufgenommen. Peros et al.[38] untersuchten den Effekt körperlicher Fitness auf die Prävalenz von MSE unter 50 Zahnmedizinstudierenden anhand von Fragebögen und dem Astrand Bicycle Ergometer Test. Sie konnten beschreiben, dass Zahnmedizinstudierende, die während des Semesters zwei Mal wöchentlich für mindestens 90 Minuten an einem universitären Trainingskurs teilnahmen, 
eine deutlich bessere körperliche Fitness hatten. Probanden die regelmäßig trainierten hatten ausßerdem weniger muskuloskelettale Beschwerden. Der universitäre Trainingskurs enthielt dabei Auswahlmöglichkeiten zwischen Teamsportarten, wie Fußball oder Basketball, individuelle Sportarten, wie Schwimmen oder Tennis, und viele mehr. In der zahnmedizinischen Literatur [5, 24, 41] sind zwar des Weiteren Aerobicübungen, Yoga und Krafttraining als adäquates Training empfohlen, allerdings ohne sich dabei auf experimentelle Daten zu stützen. Aufgrund der mangelnden Literatur bleibt trotz der nachgewiesenen positiven Effekte folglich unklar, welche Art von körperlichem Training tatsächlich für ZÄ und ZFAs empfehlenswert ist, um MSE zu vermeiden oder zu reduzieren und ein uneingeschränktes und beschwerdefreies Berufsleben zu ermöglichen. Die einzigartigen körperlichen Anforderungen des zahnmedizinischen Arbeitsumfelds erfordern vermutlich ein ebenso individuelles Training für ZÄ und ZFAs.[8] Soll die hohe MSEPrävalenz in der Zukunft gesenkt werden, erschließt sich die Forderung herauszufinden, welches Trainingsprogramm für Z̈̈ und ZFAs adäquat ist. Um wissenschaftlich fundierte Empfehlungen aussprechen zu können sind folglich weitere experimentelle Studien zum Einfluss von Körperlichen Training unter zahnmedizinischem Personal zwingend, die an den Ergebnissen dieser Studie und der ansonsten wenig verfügbaren Literatur anknüpfen.

\subsection{Material- und Methodenkritik}

\subsubsection{Probandenkollektiv}

Das Probandenkollektiv bestand aus 22 Zahnärzten, Zahnmedizinischen Fachangestellten und Studenten der Zahnmedizin. Dabei nahmen 19 Frauen und 3 Männer an der Studie teil. Eine gleichmäßige Geschlechterverteilung wäre für die Auswertung geschlechtlicher Unterschiede sinnvoll gewesen, allerdings stimmt die Geschlechterverteilung mit dem prädominant von Frauen ausgeführten Beruf des Zahnmedizinischen Fachangestellten sowie dem seit Jahren beobachteten Wandel in zahnmedizinischen Studiengängen hin zu hohen Frauenquoten überein.[10, 31, 186] In einer aktuellen Studie über Zahnmedizinische Fachangestellte waren von 406 Teilnehmern nur 1,23\% männlich.[10] In einer weiteren aktuellen Studie die die Prävalenz von MSE bei Z $\ddot{A}$ und Zahnmedizin Studenten erhob waren zwei Drittel der Probanden weiblich.[31] Das statistische Bundesamt gibt 
die Geschlechterverteilung in zahnmedizinischen Studiengängen für das Wintersemester 2015/16 mit 9674 weiblichen von insgesamt 15085 Zahnmedizinstudierenden an, für das Wintersemester 2019/20 waren es 10029 weibliche von insgesamt 15396 Studierenden.[186] Bei der Verteilung der Probanden nach Berufsgruppen waren 59,09\% der Probanden Zahnarzt/ärztin, 31,82\% Zahnmedizinische Fachangestellte und 9,09\% Studierende der Zahnmedizin. Für eine direkten Vergleich der Berufsgruppen, wäre eine höhere Probandenzahl unter den ZFAs notwendig gewesen. Das geringere Interesse an einer Krafttrainingsintervention ist ggf. mit Ergebnissen der Studie „Gesundheit in Deutschland aktuell 2012“ [187] erklärbar, welche zeigt, dass Personen höherer Bildungsgruppen ab 30 Jahren deutlich häufiger sportlich aktiv sind als in niedrigeren Bildungsgruppen.

Das Alter der Probanden lag im Mittel bei 38,52 Jahren und somit unter dem von den der Bundes Zahnärztekammer veröffentlichten Altersdurchschnitt der Mitglieder von 48,7 Jahren. [188] Für eine Analyse des Trainingseffekts nach Altersgruppen wäre eine vermehrte Anzahl älterer Probanden sinnvoll gewesen, welche aber in der Probandenakquise nicht gewonnen werden konnten. Dies könnte zurückgeführt werden auf eine erhöhte Präsenz ergonomischer Themen im Rahmen der studentischen und beruflichen Ausbildung in den letzten Jahren sowie aktueller Literatur bezüglich Berufsrisiken und Berufserkrankungen die vermehrt jüngere Z $\ddot{A}$ und ZFAs erreichen. Des Weiteren zeigen die Ergebnisse der Studie „Gesundheit in Deutschland aktuell 2012“ [187], dass die sportliche Aktivität mit dem Alter deutlich abnimmt, während 84,5\% der 18 bis 29 Jährigen sportlich aktiv sind, sind es ab 65 Jahren nur noch $51 \%$. Es ist außerdem von einem überdurchschnittlichen Gesundheitsbewusstsein der Probandenkohorte im Vergleich zur Durchschnittsbevölkerung auszugehen, da 100\% der Probanden angaben, in ihrer Freizeit sportlich aktiv zu sein.

Die Probanden waren im Mittel 169,24 cm groß und lagen damit im Schnitt über dem von Mensink et al. [189] im Rahmen der „Studie zur Gesundheit Erwachsener in Deutschland“, unter über 7000 Teilnehmern, ermittelten Mittelwert von 165,8 cm. Das Gewicht der Probanden hatte seinen Mittelwert bei 64,67 kg und lag somit knapp unter dem von Mensink et al. [189] ermittelten Mittelwert von 65,2 kg. Die Unterschiede zur „Studie zur Gesundheit Erwachsener in Deutschland“" lässt sich anhand der geringeren Probandenstärke und der sich somit stärker wiederspiegelnden deutlichen Abweichungen einiger Probanden erklären. 


\subsubsection{Biomechanischen Messungen}

Die biomechanischen Messungen vor und nach der Krafttrainingsintervention erfolgten in einer praxisnahmen Behandlungssimulation im Institut für Arbeitsmedizin, Sozialmedizin und Umweltmedizin des Universitätsklinikums Frankfurt am Main (Deutschland). Die den Probanden gestellten zahnmedizinischen Aufgaben wurden dabei immer an einem Phantomkopf durchgeführt. Dies geschah unter Einhaltung normaler Behandlungsund Hygienestandards, wie z.B. Wasserkühlung, Einstellen der Belichtung, Spülen und Absaugen. Für eine noch realitätsnähere biomechanische Messung wäre es optimal gewesen, die Probanden in ihren eigenen jeweiligen Behandlungsräumen zu vermessen, um somit einen Einfluss des Arbeitsplatzwechsels auf die Arbeitsweise ausschließen zu können. Dadurch ist aber einerseits die Gleichwertigkeit des zahnmedizinischen Inventars nichtmehr gegeben, welches Einfluss nehmen könnte auf die ergonomische Arbeitsweise der Probanden.[34-37] Andererseits wurden im Rahmen der Studie aus logistischen Gründen allerdings auch Z̈̈ und ZFAs zu Behandlerteams gepaart, die für gewöhnlich nicht in der gleichen Einrichtung arbeiteten. Somit mussten gleiche Rahmenbedingungen für alle Probanden geschafft werden. An zwei im Institut installierten zahnmedizinischenBehandlungsstühlen konnten alle vier gängigen Basiskonzepte nach Kimmel [176, 177] simuliert werden, welche sich in der Anordnung der Elemente des zahnärztlichen Arbeitsplatzes, wie z.B. den angeordneten Geräten und Schränken, unterscheiden. Alle Probanden behandelten stets im ihnen gewohnheitsmäßig vertrauten Behandlungskonzept und wurden somit unter gleichen Voraussetzungen und sehr praxisnahen Bedingungen vermessen. Um den ggf. vorhandenen Einfluss eines anderen Behandlungspartners auf die ergonomische Arbeitsweise zu minimieren wurden die gebildeten Teams für Prä- und Postmessung beibehalten. Da die meisten Probanden sich einzeln für die Studie anmeldeten, konnte so eine deutliche Mehrzahl an Probanden für die Studie gewonnen werden. In vergleichbaren Studien [11, 29, 121-123] in der Zahnmedizin wurde die Evaluation der Arbeitshaltung bzw. des ergonomischen Risikos während der Behandlung mit echten Patienten durchgeführt. Eine ergonomische Analyse am echten Patienten wäre für die Erhebung noch realitätsnäherer Daten positiv gewesen, ließ sich allerdings aufgrund diverser logistischer sowie ethischer Probleme nicht durchführen. Um die Vergleichbarkeit zwischen den Daten der Probanden herstellen zu können mussten alle Probanden ihrem Aufgabenfeld entsprechend die gleichen Behandlungen am gleichen Patientfall durchführen. Andere Autoren [11, 29, 122, 123] untersuchten die ergonomische Arbeitsweise ihrer 
Probanden während des normalen Tagesgeschäfts oder alltäglichen Behandlungen, unter anderem wurde für die entsprechenden Untersuchungen ein Arbeitstag zufällig ausgewählt. Dieses Studiendesign [11, 29, 121-123] eignet sich, um die ergonomische Arbeitsweise und die ergonomischen Risikofaktoren von Z̈̈ und ZFA in ihrem alläglichen Umfeld zu analysieren, allerdings weniger um im Prä-Post-Vergleich zwei Messungen auf ihre Unterschiede hin zu untersuchen. Es muss beachtet werden, dass jeder Patient ein menschliches Wesen und somit individuell ist, mit eigenen Ansprüchen und Besonderheiten. Die Aufgaben und somit Belastungen eines Behandlers können sich somit von Tag zu Tag und von Behandler zu Behandler stark unterscheiden, weshalb hier die Vergleichbarkeit objektiver Daten zwischen den Behandlungen und den Behandlern nicht gegeben ist. Um in diesem Fall eine Vergleichbarkeit zu gewährleisten, hätte jeder Proband am gleichen Patienten die gleiche zahnmedizinische Aufgabenstellung durchführen müssen und das jeweils vor und nach dem Interventionszeitraum. Dies ist zu irreal, weshalb sich für die Behandlungssimulation am Phantomkopf entschieden wurde. Für jede der gängigen Fachrichtungen der Zahnmedizin (Generalisten, Oralchirurgen, Endospezialisten, Kieferorthopäden) wurde ein Satz an Aufgabenstellungen erarbeitet, der die wesentlichen Aufgabenfelder repräsentativ darstellt (siehe Kapitel 3.2.2). Somit konnten für alle gleiche Voraussetzungen geschaffen werden und die Aufgabenstellung sowie das Praxisumfeld jedes Probanden wurden so genau wie möglich simuliert

\subsubsection{Messsystem und Auswertung}

Für die Untersuchung der ergonomischen Arbeitsweise wurde sich für die Kombination des inertialen Messsystems MVN BIOMECH Link der Firma XSens (Enschede, Niederlande) mit der observatorischen Methode RULA[120] entschieden. Dabei bestand grundsätzlich die Möglichkeit, dass sich verwendete Sensoren während der Messung lösen oder verschieben konnten. Außerdem bestand die Gefahr magnetischer Störeinflüsse auf die Inertialsensoren. Diese Störeinflüsse könnten die erhobenen biomechanischen Daten der Probanden und somit letztendlich die Studienergebnisse verfälscht haben. Die Validität und Reliabilität der inertialen Messsysteme wurden bereits in einigen Studien $[115,131]$ bestätigt. Robert-Lachaine et al. [131] untersuchten in ihrer Studie den Unterschied zwischen direkter, visueller Observation und der Messung mit Inertialsensoren anhand von Aufgaben der Materialhandhabung. Sie konnten dabei eine hohe Präzision der inertialen Messsysteme feststellen. Inertialsensoren sind außerdem in den letzten Jahren gegen den 
Einfluss elektromagnetischer Ablenkungen weitgehend stabil geworden.[116] Eine Vielzahl von Studien $[11,115,130,132,133]$ hat bereits verschiede Arbeitsfelder, wie z.B. auch das des Zahnarztes, erfolgreich anhand direkter Messsysteme untersucht. Um Messfehler oder Störungen dennoch auszuschließen, wurde in vorliegender Studie jede Messung vor Auswertung der Daten im so genannten „HD-Check“ kontrolliert. Die Untersucher überprüften erneut in der MVN ANALYZE SOFTWARE die korrekte Kalibrierung des Messsystems sowie überprüften alle durchgeführten Messungen auf Unregelmäßigkeiten und Störeinflüsse. Damit bei Unregelmäßigkeiten die Tätigkeiten exakt zugeordnet werden konnten, wurde der gesamte Messablauf gleichzeitig in einer Totalansicht aus zwei Blickwinkeln gefilmt. Alle kinematischen Daten wurden im Anschluss mit den Videoaufnahmen synchronisiert und erlaubten Messfehler oder Störeinflüsse präzise von korrekt aufgezeichneten Bewegungen abzugrenzen. Durch die durchgeführten Überprüfungen konnte somit weitgehend sichergestellt werden, dass keine technischen Probleme oder sonstige Messfehler die Auswertung der Daten verfälschten.

Die mit MVN BIOMECH Link gewonnenen kinematischen Rohdaten bestanden dabei i.d.R. aus sehr großen Datensätzen, welche vor der Auswertung über Stunden oder Tage prozessiert werden mussten, bevor sie ausgewertet werden konnten. Außerdem mussten für die Auswertung der biomechanischen Daten im Programm Matlab (MATrix LABoratory) die Auswertungsparameter in Programmiersprache definiert werden. Dabei waren Anpassungen des RULA-Arbeitsbogen für die automatische Auswertung notwendig, welche ebenfalls im Quellcode definiert waren. Der erhöhte Schwierigkeitsgrad und Aufwand der Studie ermöglichte den Untersuchern allerdings eine präzisere und ganzheitliche Beurteilung der ergonomischen Arbeitsweise der Probanden.[119, 135, 136]

Bei der Auswertung der kinematischen Rohdaten wurde nach der Vorlage des RULAArbeitsbogens gearbeitet. Auf dessen Grundlage wurde der Großteil der ergonomischen Arbeitsweise der Probanden als gesundheitlich hochgradig riskant eingestuft. Die observatorische Methode RULA gilt als konservativ und zeigte in Vergleichsstudien mit anderen Methoden eine erhöhte Tendenz Arbeitsplätze als eher riskant einzustufen.[117] Die Studienresultate könnten somit bei der Beurteilung mit anderen Methoden, die ggf. weniger konservativ sind, abweichen. Dabei muss die Methode so ausgewählt werden, dass die für zahnmedizinische Arbeiten relevanten Belastungen bzw. Körperregionen abgedeckt sind. Andere Studien [121, 122] in denen die ergonomische Arbeitsweise von zahnmedizinischem Personal untersucht wurde, nutzten z.B. den Quick Exposure Check 
(QEC) oder das Modified-Dental Operator Posture Assessement Instrument (M-DOPAI). Diese Studien nutzen die jeweilige Methode zusätzlich zum RULA. Partido et al.[122] untersuchten 21 ZÄ und 17 ZFAs anhand von RULA und M-DOPAI. Für das M-DOPAI erzielten die Probanden im Mittel einen Wert von 14,10 bei einer möglichen Maximalpunktzahl von 32. Für das RULA erzielten die Probanden im Mittel einen Gesamtscore von 3,26 von maximal 7 möglichen Punkten. In dieser zahnmedizinischen Studie ist demnach kein großer Unterschied zwischen der Bewertung von RULA und M-DOPAI zu beschreiben; beide evaluieren die ergonomische Arbeitsweise der Probanden insgesamt als einen mittleren Score auf der Bewertungsskala. In einer weiteren zahnmedizinischen Studie untersuchten Park et al.[121] die ergonomische Arbeitsweise von Z̈̈ anhand von RULA und dem QEC. Mit beiden observatorischen Methoden wurde das gesundheitliche Risiko für Behandlungen von Frontzähnen im Ober- und Unterkiefer als Moderat eingeschätzt. Für Behandlungen von zweiten Molaren im Ober- und Unterkiefer schätzte RULA das Risiko als hoch und der QEC das Risiko als moderat bis hoch ein. Auch diese beiden Methoden deckten sich also größtenteils in der Beurteilung der zahnmedizinischen Arbeitsweise.

Bei einer Vielzahl der gängigen observatorischen Methoden, unter Anderem dem QEC, fließt die subjektive Selbstempfindung des Arbeiters mit in die Bewertung des ergonomischen Risikos mit ein. Chiasson et al.[117] untersuchten acht der gängigsten observatorischen ERATs an 224 Arbeitsplätzen. Bei fünf der acht untersuchten Methoden floss das Kriterium der Selbstwahrnehmung des Probanden mit in die Risikobeurteilung ein. Dieses Kriterium kann dabei wichtigen Aufschluss geben auf die grundlegenden Ursachen schwerwiegender Risikofaktoren. Für eine rein objektive Beurteilung der ergonomischen Arbeitsweise ist es jedoch unerheblich, so dass ein Großteil der anderen gängigen Untersuchungsmethoden in dieser Studie keine Anwendung finden konnten. Um eine ganzheitliche und objektive Beurteilung des Arbeitsplatzes zu erzielen, musste eine passende observatorische Methode außerdem mit der direkten Messung durch Inertialsensoren kombiniert werden.[119, 129] Da die Kombination dieser beiden Werkzeuge eine innovative Art der ergonomischen Risikobeurteilung darstellt, wurde sich für eine präzise Umsetzung an aktueller Literatur orientiert. Die Arbeitsgruppe von Vignais [137] konnte zeigen, dass die Evaluation eines Arbeitsplatzes mithilfe von Inertialsensoren und RULA zu 
präzisen und verlässlichen Ergebnissen führt. Die Erkenntnisse der Arbeitsgruppe konnten genutzt werden, um für unsere Studie über ZÄ und ZFAs den RULA Arbeitsbogen auf die mittels der Sensoren erhobenen Daten anzupassen.

Dennoch erfasst RULA wie auch andere observatorische Methoden die ergonomische Arbeitsweise in Scores. Die Gesamt- und Teilscores sind dabei durch die im Arbeitsbogen bzw. die im Quellcode angepassten Parameter definiert. Dadurch entsteht das Problem, dass beispielsweise für Gelenkwinkel eine Veränderung der Gradzahl nicht zwangsläufig zu einem veränderten Parameter führen muss, wodurch sich die Veränderung dann nicht in der Risikobeurteilung ausdrücken würde. Um dieser ggf. mangelnden Sensibilität der Evaluation durch RULA entgegenzuwirken und die nötige Trennschärfe für den Prä-PostVergleich der biomechanischen Messungen herzustellen, wurden deshalb in der vorliegenden Studie zusätzlich der relative Zeitanteil der Gelenkwinkel bzw. Gelenkpositionen untersucht. Es erfolgte also neben der Beurteilung der Veränderung der RULA-Daten im Prä-Post-Vergleich auch eine Evaluation der Veränderung der reinen Gelenkwinkel und -positionen im Prä-Post-Vergleich.

\subsubsection{Interventionstraining}

Das Interventionstraining fand über einen Zeitraum von 10 bis 12 Wochen statt, während dem wöchentlich zwei Trainingseinheiten à 60 Minuten durchgeführt wurden. Über den Trainingszeitraum mussten die Probanden minimal 18 und maximal 24 Trainingseinheiten absolvieren. Die absolvierten Trainingseinheiten sowie die absolvierten Übungen und Wiederholungen wurden dabei vom betreuenden Trainer genauestens protokolliert. Zwei bis drei Trainingseinheiten pro Woche entspricht der in der in der Literatur anerkannten Belastungshäufigkeit für den optimalen Muskelaufbau.[42] Die Länge des Interventionszeitraums entspricht dabei anderen Studien [47, 48, 165], die den Zusammenhang eines Krafttrainings und der Prävalenz von MSD in belastungsverwandten Berufen untersucht haben. Ein längerer Interventionszeitraum mit möglichen biomechanischen Zwischenmessungen wäre u.U. interessant gewesen, um die mittel- und langfristigen Effekte eines Hypertrophietrainings auf die ergonomische Arbeitsweise von zahnmedizinischem Personal zu untersuchen. Die schwere gesundheitliche Lage der Bundesrepublik Deutschland aufgrund der SARS-CoV-2 (severe acute respiratory syndrome coronavirus type 2) Pandemie während des Studienzeitraums machte allerdings einen längeren Studienzeitraum unmöglich. Ein prolongiertes Interventionstraining war unter den Gesichtspunkten der 
Kontaktminimierung sowie des Gesundheitsschutzes von Probanden, Untersuchern und der Allgemeinheit nicht umsetzbar.

Neben einem längerer Trainingszeitraum wäre auch eine größere Probandenzahl von Vorteil gewesen, um die statistische Aussagekraft der Studie zu erhöhen. Die Probandenaquise gestaltete sich als sehr schwierig. Zum einen aufgrund der in der Regel hohen Arbeitsbelastung, welche ein verpflichtendes mehrwöchiges Training für eine Vielzahl der umworbenen ZÄ und ZFAs unmöglich machte. Zum anderen waren aufgrund der aktuellen gesundheitlichen Lage im Rahmen der SARS-CoV-2 Pandemie während des Interventionszeitraums weniger Probanden zur Teilnahme an der Studie bereit bzw. brachen diese vorzeitig ab.

Andere Interventionsstudien im Krafttrainingsbereich [46, 52] weisen höhere Probandenzahlen auf, unterscheiden sich dabei allerdings auch deutlich von der Art der Trainingsübungen und in der Qualität der Trainingsbetreuung. Während in vorliegender Studie jedes Training von qualifizierten und erfahrenen Trainern in Kleingruppen bis maximal vier Personen betreut wurde, um eine bestmögliche Trainingsqualität zu gewährleisten, wurden die Trainingseinheiten in anderen Studien [52] z.T. in größeren Gruppen betreut. Des Weiteren erfolgte die Betreuung durch erfahrene Trainer in anderen Studien [46, 49, 52] nur bei einem Teil der Trainingseinheiten, nur unregelmäßig oder gar nur am Anfang des Trainingszeitraums. Das Training, ob eigenverantwortlich oder betreut, wurde außerdem in vergleichbaren Untersuchungen oftmals am Arbeitsplatz oder zuhause durchgeführt und entzog sich somit zumindest teilweise der Kontrolle der Untersucher.[46, 48, 49] Die tatsächliche Trainingsbeteiligung und regelmäßige Durchführung der Übungen wurde beispielweise bei Zebis [49] durch den Probanden selbst protokolliert. Nur 85\% der Probanden gaben in dabei an das Trainingsprogramm jede Woche auszuführen, obwohl nur ein Mal pro Woche trainiert wurde. Durch die Betreuung jeder Trainingseinheit in Kleingruppen konnte in der vorliegenden Untersuchung zum einen für alle Probanden gleiche Trainingsbedingungen und eine gleichmäßig hohe Trainingsbeteiligung geschaffen werden. Zum anderen konnte eine hohe Trainingsqualität, z.B. im Sinne einer korrekten und sauberen Ausführung der Übungen, aber auch einer adäquaten Belastungssteigerung über den gesamten Trainingszeitraum durch exaktes Protokollieren und die Expertise der qualifizierten Trainer aufrecht erhalten werden. Dadurch, dass das Trainingsprogramm von 
allen Probanden in der gleichen Einrichtung und mit den gleichen Geräten sowie der gleichen Ausrüstung durchgeführt wurde konnten im Training für exakt gleiche Rahmenbedingungen gesorgt werden.

Um den Einfluss anderer körperlicher Aktivität sowie Physiotherapie oder ähnlichen Behandlungen auf die Studienergebnisse zu verhindern, wäre es theoretisch günstig gewesen die Probanden anzuweisen auf sportliche Aktivitäten sowie beschriebene Behandlungen über den Interventionszeitraum zu verzichten. Ein durch den Untersucher angeordneter Verzicht der Probanden ließe sich praktisch nicht vom Untersucher kontrollieren. Zuwiderhandelnde Probanden könnten somit trotzdem die Studienergebnisse verfälschen. Andererseits ist die Anordnung eines solchen Verzichts ethisch fragwürdig. Um die gleichen Bedingungen für alle Probanden zu schaffen, wurde auf eine solche Anweisung verzichtet.

Das Trainingsprogramm wurde nach den anerkannten Belastungsnomativa eines Hypertrophietrainings erstellt.[42] Die zu trainierenden Muskelgruppen wurden dabei nach der in der aktuellen Literatur $[6,9,10,12,26]$ vorherrschenden MSD-Prävalenz pro Region ausgewählt. Bereits Andersen [165] zeigte den reduzierenden Einfluss eines spezifischen Krafttrainings, welches direkt die betroffenen Muskelgruppen trainiert, auf die Prävalenz und Intensität von Schmerzen bei Probanden mit Trapeziusmyalgie. Im Gegensatz zu vergleichbaren Interventionsstudien [46, 48, 49, 52], in denen z.B. Freihantelübungen oder Übungen mit Kettelbelts im Trainingsprogramm vorgesehen waren, wurde in vorliegender Studie Übungen an Krafttrainingsgeräten und Übungen mit dem eigenen Körpergewicht ausgewählt. Dies geschah zum einen aufgrund des technisch geringeren Anspruchs der Übungsdurchführung an den Probanden sowie der einfacheren Standardisierung des Trainingsablaufs durch unterschiedliche Trainingsstationen in Form unterschiedlicher Geräte. Zum anderen ermöglichte ein Teil der Geräte die digitale Erfassung des 1RM des Probanden und somit einer objektiv genauen Einschätzung einer adäquaten Belastungsintensität.

\subsection{Ausblick}

Die vorliegende Studie untersuchte erstmals den Einfluss eines systematischen Krafttrainings auf die ergonomische Arbeitshaltung von Z $\ddot{A}$ und ZFAs anhand objektiver Daten. Dabei konnte anhand der gemessenen Gelenkwinkel und -positionen gezeigt werden, dass 
ein 10- bis 12-wöchiges systematisches Krafttraining die ergonomische Arbeitshaltung kaum bzw. nur in geringem Ausmaße beeinflussen kann. Wie bereits diskutiert, kommt es in den ersten Wochen eines Krafttrainings primär zur neuronalen Adaptionsvorgängen, ein Hypertrophieeffekt der Muskulatur hingegen tritt erst nach mehreren Monaten auf.[42, 184, 185] Es ist vorstellbar, dass erst morphologische Anpassungsmechanismen zu einem deutlichen posturalen Einfluss führen. Um diesen Zusammenhang zu untersuchen, könnten zukünftige Studien, aufbauend auf dem Studiendesign der vorliegenden Arbeit, den Einfluss eines systematischen Krafttrainings über einen längeren Interventionszeitraum untersuchen. Um die Relation zwischen einem Hypertrophieeffekt und posturalen Veränderungen zu verstehen, sollten außerdem vor, während und nach der Intervention u.a. die Muskelquerschnitte bzw. die Muskelmasse der Probanden gemessen werden.

Auf ähnliche Art und Weise demonstrieren die Ergebnisse, dass ein systematisches Krafttraining die Bewertung der ergonomischen Arbeitsweise nur teilweise positiv beeinflussen kann. Insgesamt verbesserte sich die Bewertung der Probanden dieser Studie nicht. Damit wiedersprechen die Studienergebnisse dieser Studie den beschriebenen Resultaten von Yiu et al.[50], die insgesamt eine Verbesserung der ergonomischen Arbeitsweise durch ein körperliches Training beschrieben. Auch wenn das Studiendesign von Yiu et al.[50] in zahlreichen Punkten, wie z.B. Probandenkohorte, Trainingsart oder Bewertung der Ergonomie, von der vorliegenden Studie abweicht, stellt sie dennoch die einzig vergleichbare Literatur dar. Sowohl in der Zahnmedizin als auch in Arbeitshaltung sowie Belastung von verwandten Berufen wird der Einfluss von verhaltenspräventiven Maßnahmen, wie körperlichem Training, fast ausschließlich bezüglich seines Einflusses auf die MSE-Prävalenz untersucht.[38, 46, 47, 49, 50, 53, 165] Die exakte Relation zwischen körperlichem Training und Körperhaltung bleibt hingegen weitgehend unklar.[50] Zwar wurde bereits mehrfach die ergonomische Arbeitsweise von Z̈̈ und ZFAs als solche untersucht, um ergonomische Risikofaktoren auszumachen; die Literatur über den Einfluss präventiver Maßnahmen auf die Arbeitshaltung ist aber bis heute lückenhaft.[11, 29, 121123, 183] Hinzu kommt, dass in der aktuellen ergonomischen Literatur beinahe nur der Einfluss verhältnispräventiver Maßnahmen untersucht wurde.[34-37, 51] Während der positive Einfluss von körperlichem Training, wie Aerobic, Yoga und Krafttraining, zwar immer wieder in der Literatur erwähnt wird, ohne sich allerdings dabei auf aktuelle experimentelle Daten aus der Zahnmedizin zu stützen.[5, 8, 24, 39-41] Zukünftige Studien 
könnten aufbauend auf dem Studiendesign dieser Studie unterschiedliche Trainingsarten überprüfen, um herauszufinden, ob diese einen posturalen Effekt auslösen. Die in der Literatur erwähnten Empfehlungen könnten so auf der Grundlage von objektiven Daten ggf. bestätigt oder widerlegt werden. Weitere experimentelle Daten zum Einfluss körperlichen Trainings sind zwingend, wenn ZÄ und ZFAs ein wissenschaftlich fundiertes Trainingsprogramm empfohlen werden können, dass diesen ein langes und beschwerdefreies Berufsleben ermöglicht. Diese Studien könnten außerdem dazu beitragen den Zusammenhang zwischen körperlichem Training und seinem Effekt auf die Körperhaltung besser zu verstehen.

Wird die Literatur $[34,35,106,108,110,111,114]$ zu verhaltens- und verhältnispräventiven Maßnahmen in der Zahnmedizin betrachtet, wird besonders der Mangel an Studien, welche sich auf objektive Daten stützen, deutlich. Objektive Daten sind allerdings subjektiven Daten insofern überlegen, da sie sich nicht durch menschliche Faktoren, wie z.B. Voreingenommenheit oder Konzentrationsschwäche, verfälschen lassen.[129] Trotz vielen Studien [34, 35, 90, 105, 106, 108, 110, 111], die die Effektivität von verhaltens- und verhältnispräventiven Maßnahmen anhand subjektiver Daten zeigen konnten, ist die MSE-Prävalenz in der Zahnmedizin unverändert hoch.[6, 10, 31] Da als Ursache der hohen MSE-Prävalenz von ZÄ und ZFAs allgemein die ergonomische Arbeitsweise als Hauptfaktor angesehen wird, stellt sich die Frage inwiefern diese Maßnahmen tatsächlich einen klinischen Effekt erzielen können.[3, 4, 6, 7, 12, 18] Es stellt sich außerdem die Frage, ob die genannten Maßnahmen überhaupt an der richtigen Stelle ansetzt und inwiefern überhaupt eine Anpassung der ergonomischen Arbeitsweise in der Zahnmedizin möglich ist. Die vorliegende Studie kann somit nicht nur dazu dienen, die ergonomische Arbeitsweise von Z $\ddot{A}$ und ZFAs besser zu verstehen und den Einfluss von körperlichem Training auf die Arbeitshaltung einzuschätzen, sondern kann mit ihrem Studiendesign auch als Vorlage für künftige Untersuchungen dienen, in denen der Einfluss verhaltensund verhältnispräventiver Maßnahmen unter praxisnahen, reproduzierbaren Bedingungen untersucht werden soll. Neben anderen Trainingsarten könnten so auch weitere verhaltenspräventive Maßnahmen, wie z.B. Ergonomieschulungen, Stretching-Übungen, 4händige Behandlungstechniken, bezüglich ihres Einflusses auf die ergonomische Arbeitsweise anhand objektiver Daten untersucht werden. Zwar konnten bereits einige Studien [4, 66, 79, 106, 108] eine verbesserte Arbeitshaltung sowie eine Reduktion der MSE- 
Prävalenz z.B. durch Ergonomieschulungen zeigen, allerdings mangelt es an experimentellen und insbesondere objektiven Daten, die diese Effektivität unterstreichen und den tatsächlichen Einfluss auf die ergonomische Arbeitsweise wiedergeben. Die genauen posturalen Effekte dieser Maßnahmen ließen sich somit erforschen und die bisherigen Studienergebnisse könnten überprüft werden. Das gleiche gilt für Studien [34-36, 110, 111] über den Einfluss ergonomischen Equipments auf die ergonomische Arbeitsweise, wie z.B. Behandlungseinheiten, Behandlerstühlen und Instrumentarium. Diese ließen sich ebenfalls anhand von objektiven Daten erneut überprüfen. Somit könnte ihre Effektivität noch genauer beurteilt und überprüft werden. Solche Studien könnten anhand objektiver Daten des Weiteren weitere Erkenntnisse bringen inwiefern eine Anpassung der ergonomischen Arbeitsweise in der Zahnmedizin tatsächlich möglich ist. 


\section{Zusammenfassung}

\subsection{Deutsch}

In der vorliegenden Studie sollte der Einfluss eines systematischen Krafttrainings auf die ergonomische Arbeitsweise und die ergonomische Risikobeurteilung von Zahnärzten und Zahnmedizinischen Fachangestellten anhand objektiver Daten untersucht werden. Dafür wurden 22 Probanden rekrutiert. An der Studie nahmen 13 ZÄ, 7 ZFAs und 2 Studenten der Zahnmedizin teil. Als Einschlusskriterium wurden Beschwerden im Rücken- und/ oder Nacken- und/oder Schulterbereich innerhalb der letzten zwölf Monaten definiert. In den Einrichtungen des Instituts für Arbeitsmedizin, Sozialmedizin und Umweltmedizin des Universitätsklinikums Frankfurt am Main (Deutschland) fanden biomechanische Präund Postmessungen der ergonomischen Arbeitsweise statt. Die Messungen erfolgten mit dem inertialen Messsystem MVN BIOMECH Link der Firma XSens (Enschede, Niederlande). Die Probanden arbeiteten während der Messung an einer ihnen vertrauten Behandlungseinheit und behandelten am Phantomkopf in einer ihrer Fachrichtung entsprechenden Behandlungssimulation bestehend aus praxisnahen zahnmedizinischen Aufgaben. Zwischen der Prä- und Postmessung absolvierten die Probanden eine 10- bis 12wöchige, systematische Krafttrainingsintervention mit zwei einstündigen Trainingseinheiten pro Woche. Für das Training wurden Übungen zur Kräftigung der stabilisierenden Haltemuskulatur ausgewählt, insbesondere im Rücken, Nacken, Schultern und Rumpf, entsprechend den vorherrschenden Regionen muskuloskelettaler Beschwerden unter Z und ZFAs.[6, 9, 10, 12, 30] Das Training wurde in Kleingruppen von den Untersuchern betreut.

Die erhobenen kinematischen Daten der biomechanischen Messungen wurden im Programm Matlab (MATrix LABoratory) auf Grundlage der gemessenen Gelenkwinkel und -positionen sowie des angepassten RULA Arbeitsbogens ausgewertet.[120] Die statistische Auswertung erfolgte mit den Programmen BiAS Version 11.12 (Epsilon Verlag, Darmstadt, Deutschland, 2020) und IBM SPSS Statistics Version 26 (IBM, New York, USA, 2019). Die erhobenen RULA Parameter wurden mit dem Wilcoxon-Matched-Pairs Test statistisch ausgewertet. Die erhobenen Gelenkwinkel wurden mithilfe des Systematic Parametric Mapping statistisch überprüft. Das Signifikanzniveau lag bei $5 \%$.

Die vorliegende Studie konnte anhand objektiver Daten die Ergebnisse vorheriger Autoren bestätigen, dass ZÄ und ZFAs oftmals mit vorgebeugtem Nacken und Oberkörper 
arbeiten, während diese z.T. zusätzlich noch rotiert und/oder seitlich gebeugt sind.[3, 4, 11-13, 33, 69] Besonders der Nacken weicht bei der Arbeit stark von einer neutralen Haltung ab. Mit den oberen Extremitäten arbeiten die Behandler oftmals asymmetrisch. Der rechte Oberarm ist stärker angehoben und abduziert als der linke und weicht stärker von einer neutralen Haltung ab. Mit den Unterarmen arbeiten Z̈̈ und ZFAs oftmals exzentrisch über die Körpermitte hinaus bzw. außerhalb ihrer Körpermitte. Die Handgelenke sind zumeist stark angewinkelt und zeigen eine deutliche Radialdeviation. Die ergonomische Arbeitsweise von zahnmedizinischem Personal konnte anhand dieser Studie als gesundheitlich hoch risikobehaftet eingestuft werden. Besonders die Nackenregion, die Unterarme sowie die beiden Handgelenke konnten anhand der objektiven Daten als Risikobereiche ausgemacht werden. Der Einfluss des systematischen Krafttrainings wurde im Prä-Post-Vergleich anhand der kinematischen Daten überprüft. Die gemessenen Gelenkwinkel und -positionen zeigten sich nach der Trainingsintervention größtenteils unverändert oder zeigten keine Verbesserung hin zu neutralen Gelenkwinkeln. Dies suggeriert, dass ein 10-12 wöchiges systematisches Krafttraining nicht oder nur begrenzt zu einer aufrechteren, entspannten und symmetrischeren Arbeitshaltung führt wie sie für die zahnmedizinischen Berufe gefordert wird.[20-25] Der Einfluss des systematischen Krafttrainings wurde ebenfalls bezüglich der ergonomische Risikobeurteilung durch RULA im Prä-Post-Vergleich überprüft. Für die Gesamtbewertung der Arbeitsweise konnte statistisch keine signifikante $(\mathrm{p} \geq 0,05)$ Veränderung und deskriptiv keine Verbesserung nachgewiesen werden. Auch im Vergleich der ergonomischen Risikobeurteilung der einzelnen Körperregionen zeigte nur der Nacken eine statistisch signifikante Veränderung ( $\mathrm{p} \leq$ 0,05). Für ihn konnte bestätigt werden, dass ein systematisches Krafttraining zu einer wenn auch geringen- verbesserten objektiven Bewertung der ergonomischen Arbeitsweise und weniger Arbeitszeit in gesundheitlich riskanten ergonomischen Arbeitsweisen führt.

Mit dieser Studie konnte anhand objektiver Daten das hohe gesundheitliche Risiko zahnmedizinischer Berufe und ihrer ergonomischen Arbeitsweise bestätigt werden. Ein systematisches 10- bis 12-wöchiges Krafttraining zeigte zumeist keinen Einfluss bzw. keine Verbesserung bezüglich der ergonomischen Arbeitsweise. Die Studienergebnisse stellen in Frage, in wie fern sich die Arbeitshaltung in zahnärztlichen Berufen durch verhaltenspräventive Maßnahmen wie körperliches Training beeinflussen lassen. 


\subsection{Englisch}

The aim of the present study was to investigate the influence of systematic strength training on the ergonomic working posture and ergonomic risk assessment of dentists and dental assistants on the basis of objective data. For this purpose, 22 subjects were recruited. 13 dentists, 7 dental assistants and 2 dental students took part in the study. The inclusion criterion was defined as complaints in the back and/or neck and/or shoulder area within the last twelve months. At the beginning of the study, each participant completed a questionnaire which collected socio-demographic data, the prevalence of musculoskeletal diseases/complaints and data on health-related activities.[54] The biomechanical preand postmeasurements of ergonomic working posture took place in the facilities of the Institute of Occupational Medicine, Social Medicine and Environmental Medicine of the University Hospital Frankfurt am Main (Germany). The kinematic measurements were carried out with the inertial measurement system MVN BIO-MECH Link from XSens (Enschede, Netherlands). During the measurement, the participants worked on a treatment unit familiar to them and treated in a treatment simulation corresponding to their speciality, consisting of dental tasks close to practice. Between pre- and postmeasurement, the subjects completed a 10- to 12-week systematic strength training intervention with two one-hour training sessions per week. Exercises to strengthen the stabilizing postural muscles were selected for the training, especially in the back, neck, shoulders and trunk, according to the predominant regions of musculoskeletal complaints among dentists and dental assistants.[6, 9, 10, 12, 30] The training was supervised in small groups by qualified sports scientists or researchers who were specially trained for the training intervention.

The collected kinematic data of the biomechanical measurements were analyzed in the Matlab program (MATrix LABoratory) based on the measured joint angles and positions as well as the adapted RULA work sheet.[120] The statistical analysis was performed with the programs BiAS Version 11.12 (Epsilon Ver-lag, Darmstadt, Germany, 2020) and IBM SPSS Statistics Version 26 (IBM, New York, USA, 2019). The collected RULA parameters were statistically tested using the Wilcoxon matched pairs test. The joint angles collected were statistically tested using Systematic Parametric Mapping. The significance level $\mathrm{p} \leq$ was defined at 0.050 .

The present study was able to confirm the results of previous studies on the basis of objective data, namely that dentists and dental assistants often work with the neck and upper 
body bent forward, while these are partly also rotated and/or bent sideways.[3, 4, 11-13, $33,69]$ The neck in particular deviates strongly from a neutral posture during work. The practitioners often work asymmetrically with the upper extremities. The right upper arm is raised and abducted more than the left and deviates more from a neutral posture. With the forearms, dentists and dental specialists often work eccentrically beyond the centre of the body or outside the centre of the body. The wrists are usually strongly bent and show a clear radial deviation. Based on this study, the ergonomic working methods of dental staff could be classified as high-risk in terms of health. In particular, the neck region, the forearms and both wrists could be identified as risk areas based on the objective data. The influence of the systematic strength training was checked in the pre-post comparison using the kinematic data. The measured joint angles and positions were mostly unchanged or showed no improvement towards neutral joint angles after the training intervention. This suggests that 10-12 weeks of systematic strength training does not, or only to a limited extent, lead to a more upright, relaxed and symmetrical working posture as required for the dental profession.[20-25] The influence of the systematic strength training was also examined with regard to the ergonomic risk assessment by RULA in a pre-post comparison. For the overall assessment of working methods, no statistically significant ( $\mathrm{p} \geq$ 0.05 ) change and descriptively no improvement could be demonstrated. In the comparison of the ergonomic risk assessment of the individual body regions, only the neck showed a statistically significant $(\mathrm{p} \leq 0.05)$ change. For the neck, it could be confirmed that systematic strength training leads to an improved objective assessment of ergonomic working practices and less working time in health-risky ergonomic working practices. With this study, the high health risk of dental professions and their ergonomic working methods could be confirmed based on objective data. A systematic 10 to 12-week strength training showed mostly no influence or improvement regarding ergonomic working practices. The study results question the extent to which the working posture in dental professions can be influenced by behavioral preventive measures such as physical training. 


\section{$7 \quad$ Literaturverzeichnis}

1. https://www.businessinsider.com/the-most-unhealthy-jobs-in-america-201511 ? $\mathrm{r}=\mathrm{US} \& \mathrm{IR}=\mathrm{T} \# 1$, The 27 Jobs That Are Most Damaging to Your Health. Business Insider 2015 - dentists-dental-surgeons-and-dental-assistants-27. Kiersz, A., (Zugriff am 16.10.2020).

2. Ohlendorf, D., et al., Constrained posture in dentistry - a kinematic analysis of dentists. BMC Musculoskelet Disord, 2017. 18(1): p. 291.

3. Valachi, B. and K. Valachi, Mechanisms leading to musculoskeletal disorders in dentistry. J Am Dent Assoc, 2003. 134(10): p. 1344-50.

4. Yamalik, N., Musculoskeletal disorders (MSDs) and dental practice Part 2. Risk factors for dentistry, magnitude of the problem, prevention, and dental ergonomics. Int Dent J, 2007. 57(1): p. 45-54.

5. Jones, A.C. and S. Forsythe, Functional training for dentistry: an exercise prescription for dental health care personnel. J Calif Dent Assoc, 2005. 33(2): p. 137-45.

6. Lietz, J., A. Kozak, and A. Nienhaus, Prevalence and occupational risk factors of musculoskeletal diseases and pain among dental professionals in Western countries: $A$ systematic literature review and meta-analysis. PLoS One, 2018. 13(12): p. e0208628.

7. Finsen, L., H. Christensen, and M. Bakke, Musculoskeletal disorders among dentists and variation in dental work. Appl Ergon, 1998. 29(2): p. 119-25.

8. Talpos-Niculescu, C., et al., The role of physical exercise in preventing musculoskeletal disorders caused by the dental profession. Timisoara Medical Journal, 2009. 60.

9. Hayes, M., D. Cockrell, and D. Smith, A systematic review of musculoskeletal disorders among dental professionals. International Journal of Dental Hygiene, 2009. 7(3): p. 159165.

10. Ohlendorf, D., et al., Prevalence of Muscular Skeletal Disorders among Qualified Dental Assistants. Int J Environ Res Public Health, 2020. 17(10).

11. Ohlendorf, D., et al., Kinematic analysis of work-related musculoskeletal loading of trunk among dentists in Germany. BMC Musculoskeletal Disorders, 2016. 17(1): p. 427.

12. Morse, T., H. Bruneau, and J. Dussetschleger, Musculoskeletal disorders of the neck and shoulder in the dental professions. Work, 2010. 35(4): p. 419-29.

13. Sanders, M.J., Preventing Work-Related MSDs in Dental Hygienists. 2004. p. 448-473.

14. Al Wazzan, K.A., et al., Back \& neck problems among dentists and dental auxiliaries. J Contemp Dent Pract, 2001. 2(3): p. 17-30.

15. Milerad, E., et al., An electromyographic study of dental work. Ergonomics, 1991. 34(7): p. 953-62.

16. Fine, L. and B. Silverstein, Musculoskeletal disorders: Work-related disorders of the neck and upper extremity. Occupational Health, Recognizing and Preventing Work-Related Disease, 1988: p. 358-370.

17. Lalumandier, J.A. and S.D. McPhee, Prevalence and risk factors of hand problems and carpal tunnel syndrome among dental hygienists. J Dent Hyg, 2001. 75(2): p. 130-4. 
18. Alexopoulos, E.C., I.C. Stathi, and F. Charizani, Prevalence of musculoskeletal disorders in dentists. BMC Musculoskelet Disord, 2004. 5: p. 16.

19. Warren, N., Causes of musculoskeletal disorders in dental hygienists and dental hygiene students: a study of combined biomechanical and psychosocial risk factors. Work, 2010. 35(4): p. 441-54.

20. ISO11226(2000)Ergonomics-Evaluation of static working postures. International Organization for Standardization, G.

21. Oene Hokwerda, R.d.R., Sandra Shaw, Adopting a healthy sitting working posture during patient treatment. https://esde.org/files/publication/2adopting_healthy_sitting_posture_during_patient_treatment.pdf (Zugriff 22.10.2020). 2006.

22. Sachan, A., et al., Ergonomics, posture and exercises - Painfree, prolong orthodontic career. Journal of Orthodontic Research, 2013. 1: p. 89.

23. Valachi, B., Practice Dentistry Pain-free: Evidence-based Strategies to Prevent Pain and Extend Your Career. 2008: Posturedontics Press.

24. Valachi, B. and K. Valachi, Preventing musculoskeletal disorders in clinical dentistry: strategies to address the mechanisms leading to musculoskeletal disorders. J Am Dent Assoc, 2003. 134(12): p. 1604-12.

25. Pîrvu, C., et al., The dentist's operating posture - ergonomic aspects. J Med Life, 2014. 7(2): p. 177-82.

26. Leggat, P.A., U. Kedjarune, and D.R. Smith, Occupational health problems in modern dentistry: a review. Ind Health, 2007. 45(5): p. 611-21.

27. Thornton, L.J., et al., Perceived musculoskeletal symptoms among dental students in the clinic work environment. Ergonomics, 2008. 51(4): p. 573-86.

28. Hayes, M., D. Cockrell, and D.R. Smith, A systematic review of musculoskeletal disorders among dental professionals. Int J Dent Hyg, 2009. 7(3): p. 159-65.

29. Rafie, F., et al., Prevalence of Upper Extremity Musculoskeletal Disorders in Dentists: Symptoms and Risk Factors. J Environ Public Health, 2015. 2015: p. 517346.

30. Hodacova, L., et al., Self-reported risk factors related to the most frequent musculoskeletal complaints among Czech dentists. Ind Health, 2015. 53(1): p. 48-55.

31. Ohlendorf, D., et al., Prevalence of Musculoskeletal Disorders among Dentists and Dental Students in Germany. International journal of environmental research and public health, 2020. 17(23): p. 8740.

32. Akesson, I., et al., Musculoskeletal disorders among female dental personnel--clinical examination and a 5-year follow-up study of symptoms. Int Arch Occup Environ Health, 1999. 72(6): p. 395-403.

33. Cherniack, M., et al., Nerve conduction and sensorineural function in dental hygienists using high frequency ultrasound handpieces. Am J Ind Med, 2006. 49(5): p. 313-26.

34. Dable, R.A., et al., Postural Assessment of Students Evaluating the Need of Ergonomic Seat and Magnification in Dentistry. J Indian Prosthodont Soc, 2014. 14(Suppl 1): p. 51-8. 
35. Plessas, A. and M. Bernardes Delgado, The role of ergonomic saddle seats and magnification loupes in the prevention of musculoskeletal disorders. A systematic review. Int J Dent Hyg, 2018. 16(4): p. 430-440.

36. Rempel, D., et al., The effects of periodontal curette handle weight and diameter on arm pain: a four-month randomized controlled trial. J Am Dent Assoc, 2012. 143(10): p. 110513.

37. Suedbeck, J.R., et al., Effects of Instrument Handle Design on Dental Hygienists' Forearm Muscle Activity During Scaling. J Dent Hyg, 2017. 91(3): p. 47-54.

38. Peros, K., et al., Physical fitness course in the dental curriculum and prevention of low back pain. J Dent Educ, 2011. 75(6): p. 761-7.

39. Lehto, T.U., H.Y. Helenius, and H.T. Alaranta, Musculoskeletal symptoms of dentists assessed by a multidisciplinary approach. Community Dent Oral Epidemiol, 1991. 19(1): p. 38-44.

40. De Sio, S., et al., Ergonomic risk and preventive measures of musculoskeletal disorders in the dentistry environment: an umbrella review. PeerJ, 2018. 6: p. e4154.

41. Nishat, R., et al., The precedence and viability of yoga in the lives of D3-dental students, dental practitioners, and dental patients. J Family Med Prim Care, 2019. 8(12): p. 38083813.

42. Wirth, K., Trainingshäufigkeit beim Hypertrophietraining, 2004.

43. ZIMMERMANN, K., Gesundheitsorientiertes Muskelkrafttraining - Theorie, Empirie, Praxisorientierung, Beiträge zur Lehre und Forschung im Sport - Band 127. Verlag Hofmann Schorndorf, Schorndorf, 2000.

44. Andersen, L., et al., Protocol for Work place adjusted Intelligent physical exercise reducing Musculoskeletal pain in Shoulder and neck (VIMS): a cluster randomized controlled trial. BMC musculoskeletal disorders, 2010. 11: p. 173.

45. Jensen, C., Development of neck and hand-wrist symptoms in relation to duration of computer use at work. Scand J Work Environ Health, 2003. 29(3): p. 197-205.

46. Blangsted, A.K., et al., One-year randomized controlled trial with different physical-activity programs to reduce musculoskeletal symptoms in the neck and shoulders among office workers. Scand J Work Environ Health, 2008. 34(1): p. 55-65.

47. Oldervoll, L.M., et al., Comparison of two physical exercise programs for the early intervention of pain in the neck, shoulders and lower back in female hospital staff. J Rehabil Med, 2001. 33(4): p. 156-61.

48. Jay, K., et al., Kettlebell training for musculoskeletal and cardiovascular health: a randomized controlled trial. Scand J Work Environ Health, 2011. 37(3): p. 196-203.

49. Zebis, M.K., et al., Implementation of neck/shoulder exercises for pain relief among industrial workers: a randomized controlled trial. BMC Musculoskelet Disord, 2011. 12: p. 205.

50. Yiu, X.Y., et al., A 10-week exercise intervention can improve work posture but not neck/shoulder symptoms in dental health students: A pilot cohort study. Work, 2020. 67(1): p. 239-249. 
51. Dong, H., et al., The effects of periodontal instrument handle design on hand muscle load and pinch force. J Am Dent Assoc, 2006. 137(8): p. 1123-30; quiz 1170.

52. Ylinen, J., et al., Active neck muscle training in the treatment of chronic neck pain in women: a randomized controlled trial. JAMA, 2003. 289(19): p. 2509-16.

53. Thiele Schwarz, U., P. Lindfors, and U. Lundberg, Health-related effects of worksite interventions involving physical exercise and reduced workhours. Scand J Work Environ Health, 2008. 34(3): p. 179-88.

54. Ohlendorf, D., et al., SOPEZ: study for the optimization of ergonomics in the dental practicemusculoskeletal disorders in dentists and dental assistants: a study protocol. Journal of Occupational Medicine and Toxicology, 2020. 15(1): p. 1-9.

55. Mutschler, E., Anatomie, Physiologie, Pathophysiologie des Menschen : 563 Abbildungen und 140 Tabellen, 2007: Stuttgart.

56. Aumüller, G., Anatomie : 208 Tabellen, in Duale Reihe2010: Stuttgart.

57. Bommas-Ebert, U., Kurzlehrbuch Anatomie und Embryologie : 46 Tabellen, 2011: Stuttgart u.a.

58. Schünke, M., E. Schulte, and U. Schumacher, Allgemeine Anatomie und Bewegungssystem : 182 Tabellen, in Prometheus : LernAtlas der Anatomie / Michael Schünke ; Erik Schulte ; Udo Schumacher. III. von Markus Voll ...2018: Stuttgart.

59. Tillmann, B.N., Atlas der Anatomie des Menschen : mit Muskeltabellen, in SpringerLehrbuch2016: Berlin, Heidelberg.

60. WHO, M.C., https://www.who.int/news-room/fact-sheets/detail/musculoskeletalconditions (Zugriff 10.11.2020).

61. Punnett, L. and D. Wegman, Work-related musculoskeletal disorders: The epidemiologic evidence and the debate. Journal of electromyography and kinesiology : official journal of the International Society of Electrophysiological Kinesiology, 2004. 14: p. 13-23.

62. Work, E.A.f.S.a.H.a., Work-related MSDs: prevalence, costs and demographics in the EU, European Risk Observatory Executive summary, 2019

63. Fuchs, J., M. Rabenberg, and C. Scheidt-Nave, Prävalenz ausgewählter muskuloskelettaler Erkrankungen: Ergebnisse der Studie zur Gesundheit Erwachsener in Deutschland (DEGS1). Bundesgesundheitsblatt - Gesundheitsforschung - Gesundheitsschutz, 2013. 56.

64. Woolf, A., J. Erwin, and L. March, The need to address the burden of musculoskeletal conditions. Best practice \& research. Clinical rheumatology, 2012. 26: p. 183-224.

65. Collaborators, G.D.B., et al., Global, regional, and national incidence, prevalence, and years lived with disability for 354 diseases and injuries for 195 countries and territories, 19902017: a systematic analysis for the Global Burden of Disease Study 2017. 2018.

66. Ratzon, N.Z., et al., Musculoskeletal symptoms among dentists in relation to work posture. Work, 2000. 15(3): p. 153-158.

67. Marklin, R.W. and K. Cherney, Working postures of dentists and dental hygienists. J Calif Dent Assoc, 2005. 33(2): p. 133-6.

68. Langford, M.L., Poor posture subjects a worker's body to muscle imbalance, nerve compression. Occup Health Saf, 1994. 63(9): p. 38-40, 42. 
69. Smith, C.A., et al., An investigation of ergonomic interventions in dental hygiene work. Appl Ergon, 2002. 33(2): p. 175-84.

70. Rundcrantz, B.L., et al., Occupational cervico-brachial disorders among dentists. Psychosocial work environment, personal harmony and life-satisfaction. Scand J Soc Med, 1991. 19(3): p. 174-80.

71. Rundcrantz, B.L., B. Johnsson, and U. Moritz, Occupational cervico-brachial disorders among dentists. Analysis of ergonomics and locomotor functions. Swed Dent J, 1991. 15(3): p. $105-15$.

72. Arbeitsmedizin, B.f.A.u., Arbeitsmedizinische Regeln 13.2 "Tätigkeiten mit wesentlich erhöhten körperlichen Belastungen mit Gesundheitsgefährdungen für das Muskel-SkelettSystem" https://www.baua.de/DE/Angebote/Rechtstexte-und-TechnischeRegeln/Regelwerk/AMR/AMR-13-2.html (Zugriff am 07.12.2020). 2014

73. Hayes, M., J. Taylor, and D. Smith, Predictors of work-related musculoskeletal disorders among dental hygienists. International journal of dental hygiene, 2011. 10: p. 265-9.

74. Lalumandier, J.A., et al., Musculoskeletal pain: prevalence, prevention, and differences among dental office personnel. Gen Dent, 2001. 49(2): p. 160-6.

75. Puriene, A., et al., General health of dentists. Literature review. Stomatologija, 2007. 9(1): p. 10-20.

76. Leggat, P.A. and D.R. Smith, Musculoskeletal disorders self-reported by dentists in Queensland, Australia. Aust Dent J, 2006. 51(4): p. 324-7.

77. Pejčić, N., et al., Assessment of risk factors and preventive measures and their relations to work-related musculoskeletal pain among dentists. Work, 2017. 57(4): p. 573-593.

78. Kierklo, A., et al., Work-related musculoskeletal disorders among dentists - a questionnaire survey. Ann Agric Environ Med, 2011. 18(1): p. 79-84.

79. Rucker, L.M. and S. Sunell, Ergonomic risk factors associated with clinical dentistry. J Calif Dent Assoc, 2002. 30(2): p. 139-48.

80. Brown, J., et al., Dental practitioners and ill health retirement: causes, outcomes and reemployment. Br Dent J, 2010. 209(5): p. E7.

81. Burke, F.J., J.R. Main, and R. Freeman, The practice of dentistry: an assessment of reasons for premature retirement. Br Dent J, 1997. 182(7): p. 250-4.

82. Zarra, T. and T. Lambrianidis, Musculoskeletal disorders amongst Greek endodontists: a national questionnaire survey. Int Endod J, 2014. 47(8): p. 791-801.

83. Ylipää, V., et al., Physical and psychosocial work environments among Swedish dental hygienists: risk indicators for musculoskeletal complaints. Swed Dent J, 1997. 21(3): p. 11120.

84. Petrén, V., et al., The relationship between working conditions and sick leave in Swedish dental hygienists. Int J Dent Hyg, 2007. 5(1): p. 27-35.

85. https://www.dguv.de/ifa/fachinfos/ergonomie/index.jsp, Institut für Arbeitschutz der Deutschen Gesetzlichen Unfallversicherung. (Zugriff am 03.12.2020).

86. https://iea.cc/what-is-ergonomics/, International Ergonomics Association. (Zugriff am 22.10.2020). 
87. Grandjean, E. Fitting the Task to the Man: A Textbook of Occupational Ergonomics. 1988.

88. Michalak-Turcotte, C., Controlling dental hygiene work-related musculoskeletal disorders: the ergonomic process. J Dent Hyg, 2000. 74(1): p. 41-8.

89. esde.org, w., European Society of Dental Ergonomics.(ESDE). Guidelines and Recommendations. . Zugriff 20.10.2018.

90. Mulimani, P., et al., Ergonomic interventions for preventing musculoskeletal disorders in dental care practitioners. Cochrane Database Syst Rev, 2018. 10(10): p. CD011261.

91. Association, A.D., An introduction to ergonomics. Risk factors, MSDs, approaches and interventions. A report of the Ergonomics and Disability Support Advisory Committee (EDSAC) to Council on Dental Practice (CDP), 2004

92. Andrews, N. and G. Vigoren, Ergonomics: muscle fatigue, posture, magnification, and illumination. Compend Contin Educ Dent, 2002. 23(3): p. 261-6, 268, 270 passim; quiz 274.

93. DIN EN 1005-4 (2009) Menschliche körperliche Leistung Teil 4: Bewertung von Körperhaltungen und Bewegungen bei der Arbeit an Maschinen. Beuth, B.

94. ISO 6385(2016) Ergonomics principles in the design of work systems. International Organization for Standardization, G.

95. Tichauer, E.R., Some aspects of stress on forearm and hand in industry. J Occup Med, 1966. 8(2): p. 63-71.

96. Chaffin, D.B., Localized muscle fatigue--definiton and measurement. J Occup Med, 1973. 15(4): p. 346-54.

97. Wilkins, E.M., Clinical practice of the dental hygienist. 2009, Philadelphia: Wolters Kluwer Health/Lippincott Williams \& Wilkins.

98. Grandjean, E., W. Hünting, and M. Pidermann, VDT workstation design: preferred settings and their effects. Hum Factors, 1983. 25(2): p. 161-75.

99. O., H., Symposium: Ergonomic principles for patient treatment. Syllabus paper. 2004.

100. Bundesgesundheitsministerium - Prävention, h.w.b.d.s.b.-v.-a.-z.p.p.h.Z.

101. Chaikumarn, M., Differences in Dentists' Working Postures When Adopting Proprioceptive Derivation vs. Conventional Concept. International journal of occupational safety and ergonomics : JOSE, 2005. 11: p. 441-9.

102. MM., B., Human-centered ergonomics: proprioceptive pathway to occupational health and peak performance in dental practice. Murphy. DC, editor. Ergonomics and the dental care worker. Washington, DC, USA: American Public Health Association; p. 275-99, 1998.

103. Callaghan, J.P. and S.M. McGill, Low back joint loading and kinematics during standing and unsupported sitting. Ergonomics, 2001. 44(3): p. 280-94.

104. Harrison, D.D., et al., Sitting biomechanics part I: review of the literature. J Manipulative Physiol Ther, 1999. 22(9): p. 594-609.

105. Finkbeiner, B.L., Four-handed dentistry revisited. J Contemp Dent Pract, 2000. 1(4): p. 7486.

106. Droeze, E.H. and H. Jonsson, Evaluation of ergonomic interventions to reduce musculoskeletal disorders of dentists in the Netherlands. Work, 2005. 25(3): p. 211-20. 
107. Faust, A.M., et al., Teaching methodologies for improving dental students' implementation of ergonomic operator and patient positioning. J Dent Educ, 2020.

108. Dehghan, N., et al., The effect of a multifaceted ergonomic intervention program on reducing musculoskeletal disorders in dentists. Med J Islam Repub Iran, 2016. 30: p. 472.

109. OSHA: Occupational Safety \& Health Administration, U.S.D.o.L.E.P., final rule, Federal Register 65:68261-68870 (2000).

110. Chang, B.J., Ergonomic benefits of surgical telescope systems: selection guidelines. J Calif Dent Assoc, 2002. 30(2): p. 161-9.

111. Branson, B.G., et al., Effect of magnification lenses on student operator posture. J Dent Educ, 2004. 68(3): p. 384-9.

112. Valachi, B., Ergonomic guidelines for selecting patient chairs and delivery systems. Dent Today, 2009. 28(7): p. 130, 132-3.

113. McLean, L., et al., Computer terminal work and the benefit of microbreaks. Appl Ergon, 2001. 32(3): p. 225-37.

114. Kumar, D.K., et al., Exercise prescriptions to prevent musculoskeletal disorders in dentists. J Clin Diagn Res, 2014. 8(7): p. ZE13-6.

115. Jun, D., et al., Are Measures of Postural Behavior Using Motion Sensors in Seated Office Workers Reliable? Human Factors: The Journal of the Human Factors and Ergonomics Society, 2019. 61: p. 001872081882127.

116. David, G.C., Ergonomic Methods for Assessing Exposure to Risk Factors for Work-Related Musculoskeletal Disorders. Occupational medicine (Oxford, England), 2005. 55: p. 190-9.

117. Chiasson, M.-È., et al., Comparing the Results of Eight Methods Used to Evaluate Risk Factors Associated with Musculoskeletal Disorders. International Journal of Industrial Ergonomics, 2012. 42: p. 478-488.

118. Winkel, J. and S. Mathiassen, Assessment of physical work load in epidemiologic studies: Concepts, issues and operational considerations. Ergonomics, 1994. 37: p. 979-88.

119. Maltry, L., et al., Präzisere ergonomische Risikobeurteilung durch die Kombination von Inertialsensoren mit observatorischen Methoden am Beispiel von RULA. Zentralblatt für Arbeitsmedizin, Arbeitsschutz und Ergonomie, 2020. 70(5): p. 236-239.

120. McAtamney, L. and E. Nigel Corlett, RULA: a survey method for the investigation of workrelated upper limb disorders. Appl Ergon, 1993. 24(2): p. 91-9.

121. Park, H.S., et al., Analysis of the risk factors of musculoskeletal disease among dentists induced by work posture. J Phys Ther Sci, 2015. 27(12): p. 3651-4.

122. Partido, B.B., R. Henderson, and H. Washington, Relationships between the postures of dentists and chairside dental assistants. J Dent Educ, 2020. 84(9): p. 1025-1031.

123. Choobineh, A., et al., Prevalence of Musculoskeletal Disorders and Posture Analysis Using RULA Method in Shiraz General Dentists in 2010. Journal of Islamic Dental Association of Iran, 2012. 2424.

124. Li, G. and P. Buckle, Current techniques for assessing physical exposure to work-related musculoskeletal risks, with emphasis on posture-based methods. Ergonomics, 1999. 42: p. 674-95. 
125. David, G., et al., The development of the Quick Exposure Check (QEC) for assessing exposure to risk factors for work-related musculoskeletal disorders. Applied ergonomics, 2008. 39: p. 57-69.

126. Lin, S., et al., Ergonomic risk exposure and work ability among young dental professionals in China: A cross-sectional study. J Occup Health, 2020. 62(1): p. e12154.

127. BAuA(2016)Volkswirtschaftliche Kosten durch Arbeitsunfähigkeit 2016. Bundesanstalt für Arbeitsschutz undArbeitsmedizin (BAuA), D.

128. BAuA (2019) Gefährdungsbeurteilung mit Leitmerkmalmethode. Bundesanstalt für ArbeitsschutzundArbeitsmedizin (BAuA), D.

129. Takala, E.P., et al., Systematic evaluation of observational methods assessing biomechanical exposures at work. Scand J Work Environ Health, 2010. 36(1): p. 3-24.

130. Patrizi, A., E. Pennestri, and P.P. Valentini, Comparison between low-cost marker-less and high-end marker-based motion capture systems for the computer-aided assessment of working ergonomics. Ergonomics, 2015. 59: p. 1-11.

131. Robert-Lachaine, X., et al., Feasibility of quantifying the physical exposure of materials handlers in the workplace with magnetic and inertial measurement units. Ergonomics, 2020. 63(3): p. 283-292.

132. İde, D., Ö. Tokçalar, and T. Gündüz, The effect of joint forces and torques on speed variation in automobile assembly lines. Work, 2018. 61: p. 1-15.

133. Battini, D., A. Persona, and F. Sgarbossa, Innovative real-time system to integrate ergonomic evaluations into warehouse design and management. Computers \& Industrial Engineering, 2014. 77.

134. Ohlendorf, D., et al., Restricted posture in dentistry - a kinematic analysis of orthodontists. BMC Musculoskelet Disord, 2017. 18(1): p. 275.

135. Plantard, P., et al., Validation of an ergonomic assessment method using Kinect data in real workplace conditions. Appl Ergon, 2017. 65: p. 562-569.

136. Manghisi, V., et al., Real time RULA assessment using Kinect v2 sensor. Applied Ergonomics, 2017. 65.

137. Vignais, N., et al., Physical risk factors identification based on body sensor network combined to videotaping. Applied ergonomics, 2017. 65.

138. Alshouibi, E.N., et al., The Effect of Number of Patients Treated, Dental Loupes Usage, Stress, and Exercise on Musculoskeletal Pain among Dentists in Jeddah. J Int Soc Prev Community Dent, 2020. 10(3): p. 336-340.

139. Gopinadh, A., et al., Ergonomics and musculoskeletal disorder: as an occupational hazard in dentistry. J Contemp Dent Pract, 2013. 14(2): p. 299-303.

140. Goldspink, G. and S. Harridge, Cellular and Molecular Aspects of Adaptation in Skeletal Muscle. 2008. p. 231-251.

141. Fukunaga, T., et al., Muscle volume is a major determinant of joint torque in humans. Acta physiologica Scandinavica, 2001. 172: p. 249-55.

142. Brechue, W. and T. Abe, The role of FFM accumulation and skeletal muscle architecture in powerlifting performance. European journal of applied physiology, 2002. 86: p. 327-36. 
143. Burger, M. and T. Burger, Neuromuscular and Hormonal Adaptations to Resistance Training: Implications for Strength Development in Female Athletes. Strength \& Conditioning Journal, 2002. 24: p. 51-59.

144. Pearson, S., et al., Muscle function in elite master weightlifters. Medicine and science in sports and exercise, 2002. 34: p. 1199-206.

145. Maughan, R., Physiology and Biochemistry of Middle Distance and Long Distance Running. 2008. p. 14-27.

146. Moss, B.M., et al., Effects of maximal effort strength training with different loads on dynamic strength, cross-sectional area, load-power and load-velocity relationships. Eur J Appl Physiol Occup Physiol, 1997. 75(3): p. 193-9.

147. Mayhew, J., et al., Relationships of Body Dimensions to Strength Performance in Novice Adolescent Male Powerlifters. Pediatric Exercise Science, 1993. 5: p. 347-356.

148. Häkkinen, K., P. Komi, and H. Kauhanen, Electromyographic and Force Production Characteristics of Leg Extensor Muscles of Elite Weight Lifters During Isometric, Concentric, and Various Stretch-Shortening Cycle Exercises. International journal of sports medicine, 1986. 7: p. 144-51.

149. Alway, S., et al., Effects of resistance training on elbow flexors of highly competitive bodybuilders. Journal of applied physiology (Bethesda, Md. : 1985), 1992. 72: p. 1512-21.

150. Kraemer, W.J., Developing a Strength Training Workout. 2008. p. 37-54.

151. BUSKIES, W.B.-B., W.-U., Gesundheitsorientiertes Fitnesstraining Band 1 Fitnessgrundlagen, Krafttraining, Beweglichkeitstraining, , 2000, Verlag Wehdemeier \& Pusch: Lüneburg.

152. EHLENZ, H.G., M.; ZIMMERMANN, E. , Krafttraining - Grundlagen / Methoden / Übungen / Leistungssteuerung / Trainingsprogramme, 2003, BLV Verlagsgesellschaft mbH: München.

153. Jones, D., D. Newham, and C. Torgan, Mechanical influence of long-lasting human muscle fatigue and delayed-onset pain. The Journal of physiology, 1989. 412: p. 415-27.

154. H, K., et al., Effects of equivolume isometric training programs comprising medium or high resistance on muscle size and strength. European journal of applied physiology, 2002. 87: p. 112-9.

155. Dons, B., et al., The effect of weight-lifting exercise related to muscle fiber cross-sectional area in humans. European journal of applied physiology and occupational physiology, 1979. 40: p. 95-106.

156. KOPYSOV, V.S., Recovery in the Training of Weightlifters. Soviet Sports Review 1979. 14 (4): p. S.202-203.

157. Robinson, J., et al., Effects of Different Weight Training Exercise/Rest Intervals on Strength, Power, and High Intensity Exercise Endurance. The Journal of Strength \& Conditioning Research, 1995. 9.

158. Touey, P., G. Sforzo, and B. McManis, 170 EFFECTS OF MANIPULATING REST PERIODS ON ISOKINETIC MUSCLE PERFORMANCE. Medicine and Science in Sports and Exercise - MED SCI SPORT EXERCISE, 1994. 26. 
159. Schlumberger, A., J. Stec, and D. Schmidtbleicher, Single- vs. Multiple-Set Strength Training in Women. Journal of strength and conditioning research / National Strength \& Conditioning Association, 2001. 15: p. 284-9.

160. Paulsen, G., D. Myklestad, and T. Raastad, The Influence of Volume of Exercise on Early Adaptations to Strength Training. Journal of strength and conditioning research / National Strength \& Conditioning Association, 2003. 17: p. 115-20.

161. Granhed, H., R. Jonson, and T. Hansson, The Loads on the Lumbar Spine During Extreme Weight Lifting. Spine, 1987. 12: p. 146-9.

162. HATFIELD, F.C., Hardcore Bodybuilding - A Scientific Approach. Contemporary Books, Lincolnwood (Chicago) 1993.

163. Pauletto, B., LET'S TALK TRAINING \#4: Rest and recuperation. National Strength \& Conditioning Association Journal, 1986. 8.

164. Baechle, T.R., R.W. Earle, and D. Wathen, Resistance training; in baechle TR, earle RW (eds): Essentials of strength training and conditioning. Champaign, Human Kinetics, 2000: p. 395425.

165. Andersen, L.L., et al., Effect of two contrasting types of physical exercise on chronic neck muscle pain. Arthritis Rheum, 2008. 59(1): p. 84-91.

166. Juul-Kristensen, B., et al., Computer users' risk factors for developing shoulder, elbow and back symptoms. Scandinavian journal of work, environment \& health, 2004. 30: p. 390-8.

167. Björksten, M.G., B. Almby, and E.S. Jansson, Hand and shoulder ailments among laboratory technicians using modern plunger-operated pipettes. Appl Ergon, 1994. 25(2): p. 88-94.

168. Eltayeb, S., et al., Work related risk factors for neck, shoulder and arms complaints: a cohort study among Dutch computer office workers. J Occup Rehabil, 2009. 19(4): p. 315-22.

169. Schomacher, J., Gütekriterien der visuellen Analogskala zur Schmerzbewertung. Physioscience, 2008. 4: p. 125-133.

170. David, G. and P. Buckle, A questionnaire survey of the ergonomic problems associated with pipettes and their usage with specific reference to work-related upper limb disorders. Appl Ergon, 1997. 28(4): p. 257-62.

171. Ylinen, J., Physical exercises and functional rehabilitation for the management of chronic neck pain. Eura Medicophys, 2007. 43(1): p. 119-32.

172. Coury, H., R. Moreira, and N. Dias, Evaluation of the effectiveness of workplace exercise in controlling neck, shoulder and low back pain: A systematic review. Revista Brasileira de Fisioterapia, 2009. 13: p. 461-479.

173. B.V., X.T., Xsens MVN User Manual. 2018.

174. Kuorinka, I., et al., Standardised Nordic questionnaires for the analysis of musculoskeletal symptoms. Appl Ergon, 1987. 18(3): p. 233-7.

175. Meyer, V.P. and W. Micheelis, Arbeitsbelastungen bei Zahnärzten in niedergelassener Praxis: eine arbeitsmedizinische Bestandsaufnahme zu Wirbelsäulenbelastungen, Berufsdermatosen und Stressfaktoren. 2001: Deutscher Zahnärzte Verl. DÄV-Hanser.

176. Kimmel, K., [The dentist's work place. Function and design--basic concepts]. ZWR, 1980. 89(3): p. 40-52. 
177. Kimmel, K., [Basic concepts. Basics of treatment room layout]. Quintessenz, 1989. 40(12): p. 2251-9.

178. https://publikationen.dguv.de/widgets/pdf/download/article/3499, DGUV Information 208-053 - Mensch und Arbeitsplatz - Physische Belastungen. (Zugriff 18.11.2020).

179. Fahrmeir, L., et al., Statistik : Der Weg zur Datenanalyse, in Springer-Lehrbuch2016: Berlin, Heidelberg.

180. Penny, W., et al., Statistical Parametric Mapping: The Analysis of Functional Brain Images. 2007.

181. Pataky, T., J. Vanrenterghem, and M. Robinson, Zero- vs. one-dimensional, parametric vs. non-parametric, and confidence interval vs. hypothesis testing procedures in onedimensional biomechanical trajectory analysis. Journal of Biomechanics, 2015. 48.

182. Pataky, T., Generalized n-dimensional biomechanical field analysis using statistical parametric mapping. Journal of biomechanics, 2010. 43: p. 1976-82.

183. Hauck, I., et al., Kinematic posture analysis of orthodontists in their daily working practice. J Orofac Orthop, 2018. 79(6): p. 389-402.

184. Sale, D., Neural Adaptation to Strength Training. 2008. p. 281-314.

185. Pearson, D., Muscle 101. Strength and Conditioning Journal - STRENGTH CONDITIONING J, 2003. 25: p. 61-62.

186. Statistisches Bundesamt, S.a.H.-F.R.-W., https://www.destatis.de/DE/Themen/Gesellschaft-Umwelt/Bildung-ForschungKultur/Hochschulen/Publikationen/Downloads-Hochschulen/studierende-hochschulen-endg-2110410207004.pdf?_blob=publicationFile (Zugriff 19.11.2020)

187. Faktenblatt zu GEDA 2012: Ergebnisse der Studie "Gesundheit in Deutschland aktuell 2012 «, h.w.r.d.D.C.G.G.G.G.S.A.p.j.F.B.D.E.

188. Deutsche Bundeszahnärztekammer - Altersverteilung, h.w.b.d.u.-u.d.-u.-z.m.a.Z.

189. Mensink, G., et al., Übergewicht und Adipositas in Deutschland, 2013, Robert Koch-Institut, Epidemiologie und Gesundheitsberichterstattung. 


\title{
8 Anhang
}

\subsection{Probanden-Information und Einwilligungserklärung}

\author{
Fachbereich Medizin / Klinikum der \\ Johann Wolfgang Goethe-Universität \\ Frankfurt am Main
}

\author{
Muskelskeletterkrankungen bei Zahnärzten und \\ Zahnmedizinischen Fachangestellten
}

\section{Probanden-Information ${ }^{1}$ und Einwilligungserklärung}

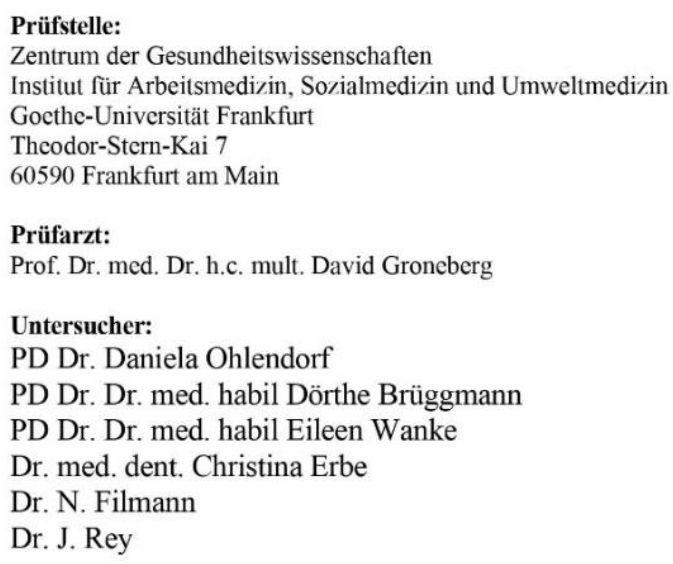

Sehr geehrte Dame, sehr geehrter Herr,

wir möchten Sie fragen, ob Sie bereit sind, an der von uns vorgesehenen Studie teilzunehmen.

Diese medizinische Studie ist notwendig, um die Ergonomie in der Zahnarztpraxis zu optimieren. Die Umfrageerhebung wird vom Institut für Arbeits-, Sozial- und Umweltmedizin der Goethe-Universität ausgehend geleitet und es sollen insgesamt mindestens 2170 Fragebögen beantwortet werden. Die biomechanische Analyse wird in den Räumlichkeiten des Instituts für Arbeits-, Sozial- und Umweltmedizin der Goethe-Universität, Theodor-SternKai 7, 60590 Frankfurt am Main durchgeführt; es sollen hierfür insgesamt ungefähr 198 Personen daran teilnehmen. Die Ergonomieschulung sowie die Anleitungen des gesundheitsorientierten, arbeitsspezifischen Krafttrainings finden ebenfalls in diesen Räumlichkeiten statt.

Die Studie wird veranlasst, organisiert und finanziert durch Prof. Dr. David Groneberg (Leiter des Instituts für Arbeits-, Sozial- und Umweltmedizin der Goethe-Universität Frankfurt

\footnotetext{
${ }^{1}$ Im Rahmen dieses Textes schließt die männliche Bezeichnung stets die weibliche Bezeichnung mit ein.
} 


\title{
Fachbereich Medizin / Klinikum der Johann Wolfgang Goethe-Universität \\ Frankfurt am Main
}

\begin{abstract}
Theodor-Stern-Kai 7, 60590 Frankfurt am Main), den Auftraggeber dieser Studie. Die Untersuchung wird durchgeführt von den Untersucherinnen PD Dr. Daniela Ohlendorf, PD Dr. Dr. med. habil Dörthe Brüggmann, PD Dr.med. Dr. med. habil Eileen Wanke (Institut für Arbeits-, Sozial- und Umweltmedizin der Goethe-Universität Frankfurt, Theodor-Stern-Kai 7, 60590 Frankfurt am Main) und Dr. med. dent. Christina Erbe (Poliklinik für Kieferorthopädie der Johannes Gutenberg Universität Mainz, Augustusplatz 2, 55131 Mainz).

Ihre Teilnahme an dieser Vermessung ist freiwillig. Sie werden in diese Studie also nur dann einbezogen, wenn Sie dazu schriftlich Ihre Einwilligung erklären. Sofern Sie nicht teilnehmen oder später aus ihr ausscheiden möchten, erwachsen Ihnen daraus keine Nachteile.

Die Untersucherinnen haben Ihnen bereits eine Reihe von Informationen zu der geplanten Studie gegeben. Der nachfolgende Text soll Ihnen die Ziele und den Ablauf der Umfrageerhebung und der biomechanischen Analyse erläutern. Bitte zögern Sie nicht, alle Punkte anzusprechen, die Ihnen unklar sind. Sie werden danach ausreichend Bedenkzeit erhalten, um über Ihre Teilnahme zu entscheiden.
\end{abstract}

\section{Warum wird diese Studie durchgeführt?}

Diese Studie wird durchgeführt, um herauszufinden, welche Aspekte der zahnmedizinischen Behandlung von ergonomischer Sichtweise verbessert werden können.

Die Umfrageerhebung erfasst alle Angaben über Beschwerden am Muskel-Skelett-System unter besonderer Berücksichtigung der Dauer und der Intensität der Beschwerden. Ferner sollen diese Angaben in Korrelation zu Arbeitsgewohnheiten gesehen werden. So können Informationen zu Belastungen des Muskel-Skelett-Systems im Bereich der Zahnmedizin gesammelt werden.

Die biomechanische Analyse soll dafür genutzt werden um die beste Arbeitsweise im Konzeptvergleich der Arbeitsplatzanordnung herauszufinden. Ferner soll die Unterteilung der zahnmedizinischen Spezialisierung dazu dienen spezialisierungsadäquate Fehlbelastungen herauszufiltern. Die Interventionen der Ergonomieschulung und des Kraftrainings sollen aktuelle Präventionsmaßnahmen hinsichtlich ihrer Effektivität analysieren.

Daraus sollen sich präventive bzw. rehabilitative Verhaltens- und Verhältnismaßnahmen formuliert werden um diesen erfolgreich entgegen zu wirken. Dadurch wird ein aktueller Überblick über Beschwerden dieses Berufsfeldes gegeben. 


\section{Fachbereich Medizin / Klinikum der Johann Wolfgang Goethe-Universität \\ Frankfurt am Main}

\section{Wie ist der Ablauf der Studie und was müssen Sie bei Teilnahme beachten?}

Die Umfrageerhebung dauert ca. 10 Minuten und erfasst alle Angaben über Beschwerden am Muskel-Skelett-System unter besonderer Berücksichtigung der Dauer und der Intensität der Beschwerden. Ferner sollen diese Angaben in Korrelation zu Arbeitsgewohnheiten gesehen werden.

Die Messdauer erfolgt über die gesamte Messzeit von 4h zusätzlich der benötigten Zeit zum Anbringen des Messequipments und der Einweisung in den Messablauf.

Vor Messbeginn werden die Sohlen der plantaren Druckverteilung in die Arbeitsschuhe eingelegt und der Messanzug mit den Sensoren angezogen. Bevor sich die Probanden auf den Arbeitsstuhl setzen wird die Druckmessmatte auf diesem platziert. Unter dem Messanzug sollen Sie enge Sportbekleidung tragen. Zusätzlich werden die durchgeführten Tätigkeiten während der Vermessung mittels der objektiven Tätigkeitsanalyse von einem Beobachter dokumentiert als auch videotechnisch aufgezeichnet.

Die Ergonomieschulung ist eine separate Einheit und geht über eine Dauer von 14 Wochen (2SWS) und ist als Vorlesung mit praktischer Übung zu verstehen.

Das gesundheitsorientierte, arbeitsspezifische Krafttraining ist auf 12 Wochen unter spezifischer Anleitung angesetzt und umfasst Trainingseinheiten von 2x/Woche à 60 Minuten.

\section{Welchen persönlichen Nutzen haben Sie von der Teilnahme an der Studie?}

Durch die Teilnahme an der Umfrageerhebung können Sie nach der Auswertung sehen, inwiefern ihre eigenen möglichen Beschwerden in ihr (angestrebtes) Berufsbild passen. Auf diese Weise können Sie eigenständig, wenn gewünscht mit Unterstützung der Untersucherinnen, präventive Maßnahmen ableiten.

Durch die Teilnahme an der Biomechanischen Analyse an der Studie bekommen Sie eine genaue Analyse ihrer Arbeitstätigkeiten, so dass Sie präventiv Bewegungen möglicherweise verbessern und Haltungen vermeiden, die große Gelenkwinkel beinhalten. Ferner erhalten Sie die Aussage darüber, an welchem Basiskonzept sie am besten, i.S. der Auswertungskriterien arbeiten.

Es ist jedoch auch möglich, dass Sie durch Ihre Teilnahme an dieser Studie keinen direkten Nutzen für Ihre Gesundheit haben.

Das Ergebnis dieser Studie könnte aber dazu beitragen, dass für andere Personen das Risiko berufsbedingter muskelskelettärer Beschwerden gesenkt werden kann, da eine Umgestaltung des Arbeitsplatzes aus Ihren Ergebnissen resultieren könnte 


\section{Fachbereich Medizin / Klinikum der Johann Wolfgang Goethe-Universität \\ Frankfurt am Main}

\section{Welche Risiken sind mit der Teilnahme an der Studie verbunden?}

Bei dem Ausfüllen des Fragebogens entstehen keine Risiken.

Das Tragen des XSens-Systems kann, wegen des zusätzlichen Gewichts (ca. 1,5 kg) und erhöhter Wärmeisolation durch Tragen des Messanzuges zu erhöhter körperlicher Anstrengung bzw. zu erhöhter Körpertemperatur/ Wärmeregulation führen.

5. Entstehen für Sie Kosten durch die Teilnahme an der Studie? Erhalten Sie eine Aufwandsentschädigung?

Es entstehen keine Kosten für Sie, da der zeitliche Aufwand der Studienteilnahme zu gering ist.

Biomechanische Analyse: Nach Auswertung Ihrer Daten erhalten Sie eine individuelle Analyse Ihrer Körperhaltung und ihrer Bewegungen (Bewegungsausmaß, -umfang und -intensität der jeweiligen statischen bzw. dynamischen Aktivitäten). Für die Teilnahme an der Studie sind Sie nicht versichert.

\section{Wer entscheidet, ob Sie aus der Studie ausscheiden?}

Sie können jederzeit, auch ohne Angabe von Gründen, Ihre Teilnahme beenden, ohne dass Ihnen dadurch irgendwelche Nachteile entstehen.

\section{Was geschieht mit Ihren Daten?}

Vor der Teilnahme sollten Sie persönlichen Daten angeben wie der vollständige Name und Alter sowie der Berufsstatus. Diese Daten werden niedergeschrieben sowie elektronisch auf den Rechnern des Instituts für Arbeits-, Sozial- und Umweltmedizin für 10 Jahre gespeichert. Die für die statistische Prüfung wichtigen Daten werden zusätzlich in pseudonymisierter Form gespeichert, ausgewertet und gegebenenfalls an Dritte zur statistischen Auswertung weitergegeben. Pseudonymisiert bedeutet, dass keine Angaben von Namen oder Initialen verwendet werden, sondern nur ein Nummern- und/oder Buchstabencode, evtl. mit Angabe des Geburtsjahres.

Die Daten werden in pseudonymisierter Form verarbeitet und anonymisiert publiziert. 


\section{Fachbereich Medizin / Klinikum der Johann Wolfgang Goethe-Universität \\ Frankfurt am Main}

8. An wen wenden Sie sich bei weiteren Fragen?

\section{Beratungsgespräche an der Prüfstelle}

Sie haben stets die Gelegenheit zu weiteren Beratungsgesprächen mit dem auf Seite 1 genannten Prüfarzt oder der Untersucherin, um weitere Fragen im Zusammenhang mit der Studie zu klären. Auch Fragen, die Ihre Rechte und Pflichten als Proband und Teilnehmer an der Studie betreffen, werden gerne beantwortet.

Wenden Sie sich bei Fragen an Frau PD Dr. Daniela Ohlendorf(Tel: 0696301 5622).

\section{Prïfstelle:}

Zentrum der Gesundheitswissenschaften

Institut für Arbeitsmedizin, Sozialmedizin und Umweltmedizin

Goethe-Universität Frankfurt

Theodor-Stern-Kai 7

60590 Frankfurt am Main

Prüfarzt:

Prof. Dr. med. Dr. h.c. mult. David Groneberg

Untersucherinnen:

PD Dr. Daniela Ohlendorf

PD Dr. Dr. med. habil Dörthe Brüggmann

PD Dr. Dr. med. habil Eileen Wanke

Dr. med. dent. Christina Erbe 


\title{
Fachbereich Medizin / Klinikum der Johann Wolfgang Goethe-Universität \\ Frankfurt am Main
}

\author{
Muskelskeletterkrankungen bei Zahnärzten und \\ Zahnmedizinischen Fachangestellten
}

\section{Einwilligungserklärung}

Name des Probanden in Druckbuchstaben

geb. am

Teilnehmer-Nr.

Ich bin in einem persönlichen Gespräch mit dem Prüfarzt und den Untersucherinnen ausführlich und verständlich über die Durchführung der Untersuchung sowie über Wesen, Bedeutung, Risiken und Tragweite der Studie aufgeklärt worden. Ich habe darüber hinaus den Text der Probandeninformation gelesen und verstanden. Ich hatte die Gelegenheit, mit dem Prüfarzt/ der Untersucherin über die Durchführung der Studie zu sprechen. Alle meine Fragen wurden zufrieden stellend beantwortet.

Möglichkeit zur Dokumentation zusätzlicher Fragen seitens des Probanden oder sonstiger Aspekte des Aufklärungsgesprächs:

Ich hatte ausreichend Zeit, mich zu entscheiden. Ich erkläre mich bereit, an der oben genannten Studie freiwillig teilzunehmen.

\section{Datenschutz:}

Mir ist bekannt, dass bei dieser Studie personenbezogene Daten erhoben, gespeichert und ausgewertet werden sollen. Die Verwendung der Angaben über meine Gesundheit erfolgt nach gesetzlichen Bestimmungen und setzt vor der Teilnahme an der Untersuchung folgende freiwillig abgegebene Einwilligungserklärung voraus, das heißt ohne die nachfolgende Einwilligung kann ich nicht an der Studic teilnehmen.

1. Ich erkläre mich damit einverstanden, dass im Rahmen dieser Studie personenbezogene Daten über mich erhoben und in Papierform sowie auf elektronischen Datenträgern im Institut für Arbeits-, Sozial- und Umweltmedizin, Klinikum der 


\section{Fachbereich Medizin / Klinikum der Johann Wolfgang Goethe-Universität \\ Frankfurt am Main}

Goethe Universität Frankfurt, Theodor-Stern-Kai 7 in 60590 Frankfurt aufgezeichnet werden. Soweit erforderlich, dürfen die erhobenen Daten pseudonymisiert (verschlüsselt) weitergegeben werden:

a) an das Institut für Biostatistik, Klinikum der Goethe Universität Frankfurt, eine beauftragte Stelle zum Zwecke de wissenschaftlichen Auswertung.

2. Ich bin darüber aufgeklärt worden, dass ich jederzeit die Teilnahme an der Studie beenden kann. Beim Widerruf meiner Einwilligung, an der Studie teilzunehmen, habe ich das Recht, die Löschung aller meiner bis dahin gespeicherten personenbezogenen Daten zu verlangen.

3. Ich erkläre mich damit einverstanden, dass meine Daten nach Beendigung oder Abbruch der Prüfung mindestens zehn Jahre aufbewahrt werden. Danach werden meine personenbezogenen Daten gelöscht, soweit nicht gesetzliche, satzungsmäßig oder vertragliche Aufbewahrungsfristen entgegenstehen.

Ein Exemplar der Probanden-Information und der Einwilligungsbestätigung habe ich erhalten Jeweils ein Exemplar verbleibt im Prüfzentrum.

Name des Probanden in Druckbuchstaben

Datum $\quad$ Unterschrift des Probanden

Ich habe das Aufklärungsgespräch geführt und die Einwilligung des Probanden eingeholt.

Name des Prüfarztes/der Prüfärztin in Druckbuchstaben

Datum

Unterschrift des aufklärenden

Prüfarztes/der Prüfärztin 


\section{$9 \quad$ Schriftliche Erklärung}

Ich erkläre ehrenwörtlich, dass ich die dem Fachbereich Medizin der Johann Wolfgang Goethe-Universität Frankfurt am Main zur Promotionsprüfung eingereichte Dissertation mit dem Titel

Der Einfluss eines systematischen Krafttrainings auf die objektive ergonomische Arbeitsweise von Zahnärzten und Zahnmedizinischen Fachangestellten

Im Institut für Arbeits-, Sozial- und Umweltmedizin des Universitätsklinikums Frankfurt am Main unter Betreuung und Anleitung von PD Dr. Dr. Daniela Ohlendorf mit Unterstützung von Dr. Fabian Holzgreve ohne sonstige Hilfe selbst durchgeführt und bei der Abfassung der Arbeit keine anderen als die in der Dissertation angeführten Hilfsmittel benutzt habe. Darüber hinaus versichere ich, nicht die Hilfe einer kommerziellen Promotionsvermittlung in Anspruch genommen zu haben.

Ich habe bisher an keiner in- oder ausländischen Universität ein Gesuch um Zulassung zur Promotion eingereicht. Die vorliegende Arbeit wurde bisher nicht als Dissertation eingereicht.

Vorliegende Ergebnisse der Arbeit wurden (oder werden) in folgendem Publikationsorgan veröffentlicht: keinen

(Ort, Datum)

(Unterschrift) 